\title{
Fetal growth restriction
}

Citation for published version (APA):

Hendrix, M. L. E. (2021). Fetal growth restriction: observation over time. [Doctoral Thesis, Maastricht University]. Maastricht University. https://doi.org/10.26481/dis.20210924mh

Document status and date:

Published: 01/01/2021

DOI:

10.26481/dis.20210924mh

Document Version:

Publisher's PDF, also known as Version of record

\section{Please check the document version of this publication:}

- A submitted manuscript is the version of the article upon submission and before peer-review. There can be important differences between the submitted version and the official published version of record.

People interested in the research are advised to contact the author for the final version of the publication, or visit the DOI to the publisher's website.

- The final author version and the galley proof are versions of the publication after peer review.

- The final published version features the final layout of the paper including the volume, issue and page numbers.

Link to publication

\footnotetext{
General rights rights.

- You may freely distribute the URL identifying the publication in the public portal. please follow below link for the End User Agreement:

www.umlib.nl/taverne-license

Take down policy

If you believe that this document breaches copyright please contact us at:

repository@maastrichtuniversity.nl

providing details and we will investigate your claim.
}

Copyright and moral rights for the publications made accessible in the public portal are retained by the authors and/or other copyright owners and it is a condition of accessing publications that users recognise and abide by the legal requirements associated with these

- Users may download and print one copy of any publication from the public portal for the purpose of private study or research.

- You may not further distribute the material or use it for any profit-making activity or commercial gain

If the publication is distributed under the terms of Article $25 \mathrm{fa}$ of the Dutch Copyright Act, indicated by the "Taverne" license above, 


\section{Fetal Growth Restriction Observation over Time}

Manouk Leonard Elisabeth Hendrix 
Design cover and chapter pages: Juliëtte Linskens Layout and print: Optima Grafische Communicatie

ISBN: 978-94-6361-576-1

(c) Copyright Manouk Hendrix, Maastricht 2021 


\section{Fetal Growth Restriction \\ Observation over Time}

\section{PROEFSCHRIFT}

Ter verkrijging van de graad van doctor aan de Universiteit Maastricht, op gezag van de Rector Magnificus, Prof. dr. R.M. Letschert, volgens het besluit van het College van Decanen, in het openbaar te verdedigen op vrijdag 24 september 2021 om 14:00 uur

door

Manouk Leonard Elisabeth Hendrix 


\section{Promotor}

Prof. dr. M.E.A. Spaanderman

\section{Copromotor}

Dr. S. Al-Nasiry

Dr. J.A.P. Bons

\section{Beoordelingscommissie}

Prof. dr. J.G. Nijhuis (voorzitter)

Prof. dr. R.F.P.M. Kruitwagen

Prof. dr. S.A. Scherjon (Universitair Medisch Centrum Groningen)

Prof. dr. C.G.J. Sweep (Radboud Universitair Medisch Centrum)

Prof. dr. L.J.I. Zimmermann 


\section{Table of contents}

Chapter 1 General introduction and outline 7

Chapter 2 Reduced fetal growth velocities and the association with neonatal outcomes in appropriate-for-gestational-age neonates: a retrospective cohort study

BMC Pregnancy Childbirth. 2019; 19(1):31

Chapter 3 Can fetal growth velocity and first trimester maternal biomarkers improve the prediction of SGA and adverse neonatal outcome?

Fetal Diagnosis and Therapy. 2019;46(4):274-284

Chapter 4 Longitudinal changes in placental biomarkers in women with early versus late placental dysfunction Hypertension in Pregnancy. 2019;38(4):268-277

Chapter 5 Role of sFlt-1 and PlGF in the screening of small-for-gesta85 tional age neonates during pregnancy: a systematic review Annals of Clinical Biochemistry. 2020;57(1):44-58

Chapter 6 Maternal vascular malformation in the placenta is an indicator for fetal growth restriction irrespective of neonatal birthweight

Placenta. 2019;87:8-15

Chapter 7 First trimester prediction of term SGA and reduced fetal growth velocity is poor

Submitted

Chapter 8 Postnatal growth during the first five years of life in SGA and AGA neonates with reduced fetal growth

Early Human Development. 2020 Dec;151:105199

Chapter 9 General discussion

Chapter 10 Summary

Chapter 11 Nederlandse samenvatting 203

Chapter 12 Impact paragraaf 211

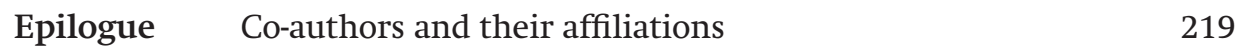

List of publications $\quad 223$

Dankwoord 227

$\begin{array}{ll}\text { Curriculum Vitae } & 231\end{array}$ 
c) 


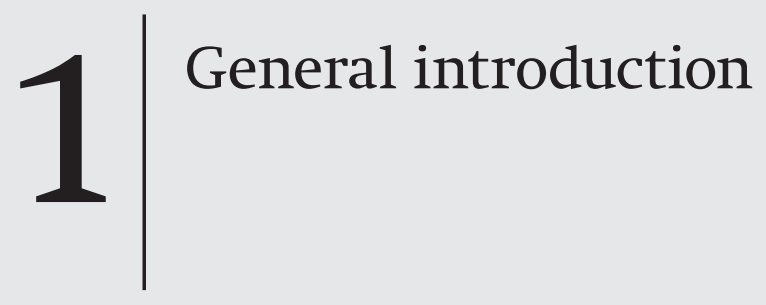



Fetal growth is important in many aspects of perinatology. Fetal growth restriction (FGR) is described as a fetus who will not reach its genetically-programmed growth potential during gestation and it affects about $10 \%$ of all fetuses during pregnancy [1]. FGR is associated with an increased risk of perinatal morbidity and mortality [2]. Prenatal care mostly focuses on detecting small-for-gestational-age fetuses (usually defined as growth below the $10^{\text {th }}$ centile) after which intensive monitoring and sometimes interventions are instituted. Most of these infants are nevertheless not growth restricted, while fetuses with a birthweight above the $10^{\text {th }}$ centile can be growth restricted. Long-term health outcomes of fetuses with FGR include impaired neurological and cognitive development and cardiovascular and endocrine diseases in adulthood [3]. It is therefore crucial to detect FGR early in pregnancy in order to reduce mortality and morbidity rates. Unraveling of the underlying cause of FGR is important for suitable monitoring and intervention strategies.

\section{Historical monitoring of fetal growth}

The oldest clinical method in the Netherlands to diagnose FGR is abdominal palpation using the Leopold maneuver [4]. In low risk pregnancies, estimating fetal weight with this maneuver is a non-invasive, practical and simple tool to estimate fetal weight as regular care. However, the diagnostic performance of detecting FGR using abdominal palpation is of limited value with low sensitivities in the range of $30-50 \%[5,6]$. The most used non-invasive tool for detecting FGR is the symphysis-fundal height tape measurement, with sensitivities of 56-86\% worldwide [7, 8]. With the advance of ultrasound techniques the crown-rump length could be introduced as a more sensitive method to determine gestational age during first trimester. The main tool for fetal size, during second and third trimester is ultrasound to establish the estimated fetal weight (EFW) by applying the Hadlock's formula [9]. From various biometric measurements the head circumference, abdominal circumference and femur formula of Hadlock gives approximately a mean deviation between EFW and birth weight of $+/-10 \%$ [10] In case of brain-sparing, the head circumference is often secured at the expense of intestinal and renal growth and as a consequence, the abdominal circumference $(\mathrm{AC})$ will be the first biometric index to be affected. This is attributed to the preferential shunting of blood to vital organs due to uteroplacental insufficiency [11] and therefore could lead to an earlier drop in AC compared with EFW [12].

Dutch guidelines do not recommend serial fetal size assessment in low risk pregnancies and therefore cross sectional measurements are commonly used to diagnose FGR. If FGR is accompanied by suboptimal increase in size, one would expect that repeated biometrical measurements between second and third tri- 
mester would provide the necessary information to diagnose FGR [13]. The use of fetal growth velocity as a predictor for FGR is one of the main topics in this thesis.

\section{Current definition of fetal growth restriction}

During the last years it has become obvious that the current definition of FGR is not conclusive. For a long time, birthweight was the most common quantitative measurement available for evaluating fetal growth, although it is actually a measurement of size [14]. Classification systems were developed based on these birthweights percentiles [15]. In general, neonates with a birthweight below the $10^{\text {th }}$ centile are classified as small-for-gestational-age (SGA), while neonates with birth weights between the 10th and 90th centiles as appropriate for gestational age (AGA). Neonates with a birthweight above the 90th centile are classified as large for gestational age (LGA) (Figure 1).

Antenatally, the detection of a small fetus is based on ultrasound parameters of fetal growth, most commonly estimated fetal weight (EFW) and abdominal circumference (AC) [16]. Traditionally, EFW and AC are plotted against different population curves. The individuals included are for many constructed curves

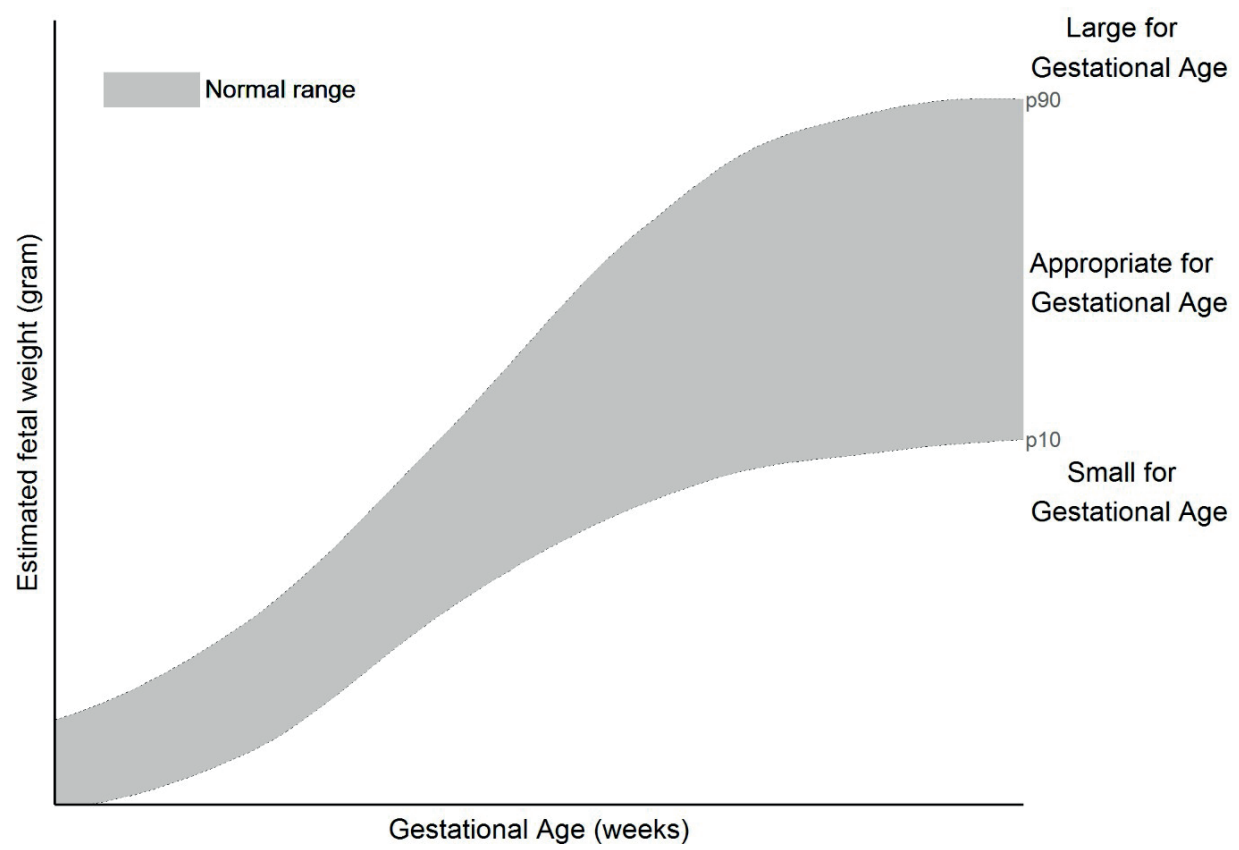

Figure 1 Estimated fetal weight during gestational age. Estimated fetal weight (EFW) divided in three groups, LGA, large-for-gestational age, AGA, appropriate-for-gestational age, SGA, smallfor-gestational age. The $\mathrm{x}$-axis presents gestational age (GA) in weeks. The y-axis presents EFW in gram. 
continuous subject of debate. FGR is most commonly defined as EFW or AC values below the 3rd, 5th or 10th centile [17].

SGA is the term used postnatally to describe a newborn with an abnormal low birth weight for a specific gestational period using variable cut-off points in literature: 3rd, 5th but mostly 10th centile. The term SGA has also been inappropriately used antenatally to define fetus with mild to moderate "smallness" (EFW or AC lower than 10th centile $[18,19]$. These definitions are based on cut off points and statistical assumptions derived from epidemiological data, rather than being derived from system-biological mechanisms. It is conceivable that the mechanism causing restriction in fetal growth can have variable severity, leading to a group of fetuses with significant decrease in growth velocity but with an estimated fetal weight still above the 10th centile. Hence, these fetuses with restricted growth are falsely considered appropriate for gestational age (AGA). These fetuses may remain undiagnosed even though they may be at increased risk for adverse outcome, in contrast, 50-70\% of SGA fetuses are constitutionally small with normal perinatal outcomes [1, 20, 21]. Undetected FGR is the strongest risk factor for stillbirth [22]. If detected and appropriately managed, the risk of stillbirth is 20-fold lower than those remaining undetected [23]. Improving detection rate is therefore important for reducing the rate of stillbirth [24, 25].

\section{Placental development contributing to fetal growth restriction}

During pregnancy, fetus and placenta form a unit, with the placenta playing a key role in fetal growth [26]. Previous studies have shown that a small placental surface area and low placental weight are associated with a slower growth of the fetus [27]. The main structural and functional units of the placenta are the chorionic villi [28]. Chorionic villi are composed of a tree-like structure with mesenchymal core, covered by trophoblast cells. The term 'trophoblast' is used to describe cells that transport nutrients and form the protective barrier between mother and fetus [29]. There are different trophoblast cells which perform different functions. The syncytiotrophoblast (SCT) is the outer lining of the placental villus that is in direct contact with maternal blood flow in the intervillous space. It forms the principal site of maternal/fetal exchange of gases and nutrients for the growth of the fetal-placental unit. The SCT membranes have microvilli which are rich in receptors for growth factors and hormones. The mononuclear villous trophoblast (VCT) lies beneath the SCT on a basement membrane and these cells are mitotic and express proliferative markers [30] (Figure 2). As the placenta enlarges, the cytotrophoblast shell becomes discontinuous and cytotrophoblast cell columns (CCCs) emerge from the distal tips of the anchoring villi in contact with the decidua. 
The extravillous trophoblast (EVT) migrate to the decidua along two differentiation pathways: the endovascular EVT moves down the inside of the spiral arteries [32], while the interstitial EVT migrate through the decidual stroma towards the maternal spiral arteries, to form a cuff of surrounding cells. This is associated with loss of actin in smooth muscle cells of the arterial media, resulting in fibrinoid change [33].

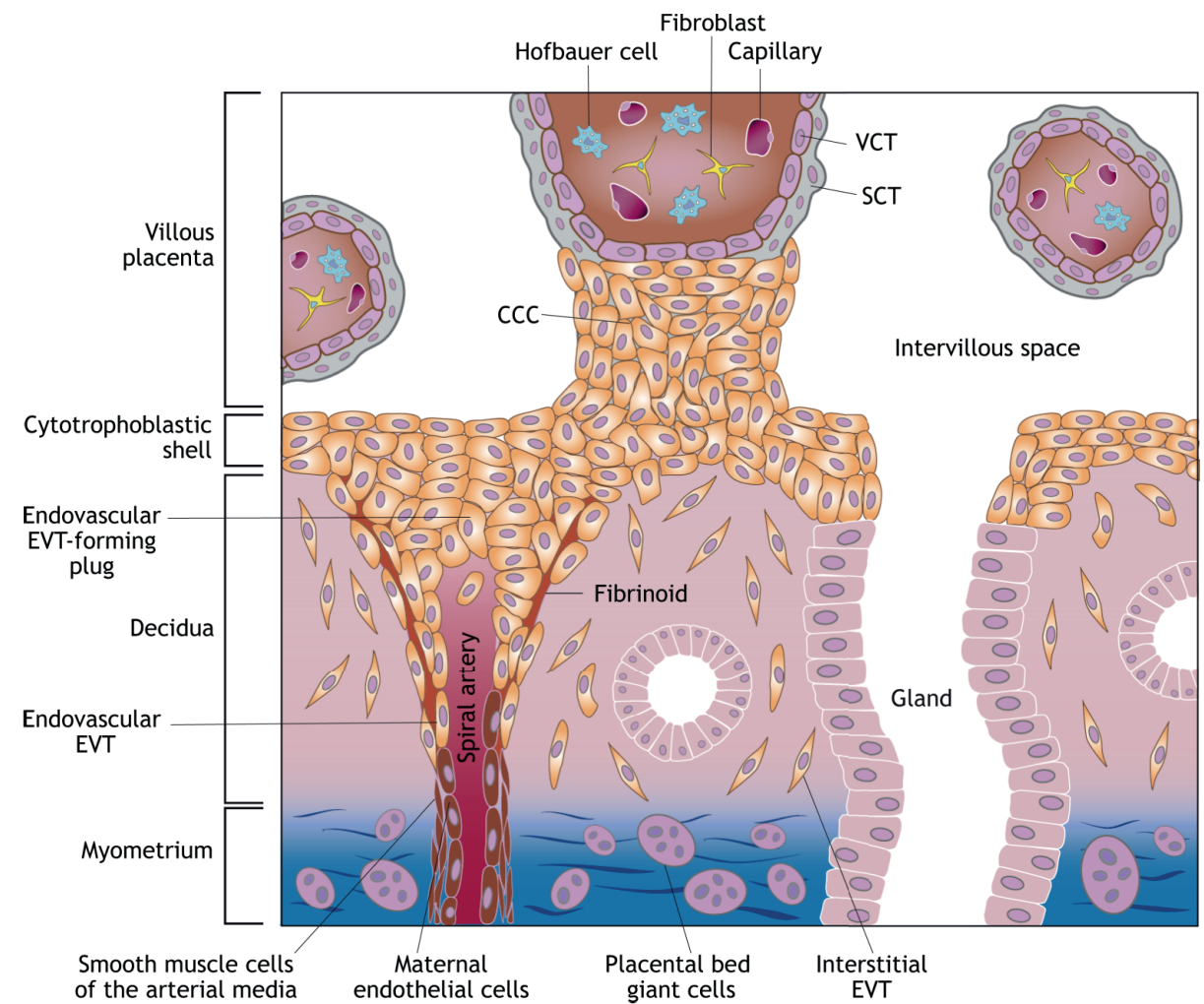

Figure 2 adapted from 'Development of the human placenta' [31].

Cells contained within the villi of the early first trimester placenta and the major trophoblast subtypes in relation to the decidua are represented. The decidual region has been illustrated to include the myometrium. Syncytiotrophoblast (SCT, grey), villous cytotrophoblast (VCT, pink), the cytotrophoblast cell column (CCC) and extra villous trophoblast (EVT) populations (endovascular and interstitial EVT, orange) are indicated. The endpoint of EVT differentiation, placental bed giant cells, are also indicated.

This trophoblast mediated transformation of the artery results in loss of vasoactivity and conversion into a vessel characterized by high blood flow conductance at low pressure. This is considered an essential adjustment for healthy pregnancy $[32,34]$. After the arterial transformation occurs, the endovascular EVT forms a 
temporarily plug that prevents blood entering the intervillous space [35]. Subsequently, the fetal cells replace the maternal endothelium and part of the smooth muscle wall transforming spiral arteries into larger low-resistance vessels capable of transporting the increased maternal blood flow to the placenta at low pressure. Normally this process encompasses the proportion of the uterine arterioles that span the decidua and the inner third of the myometrium. The decidual part of the spiral arteries is invaded between week 8 and 12 of gestation, the myometrium around mid-pregnancy.

If the arteries are not sufficiently converted and hold their contractile media, there is a disordered perfusion of blood flow into the intervillous space. This, together with an inadequate supply of nutrients and oxygen, reduces the progressive branching of the villous tree as gestation proceeds, which results in an adequate surface area available for exchange. Defective trophoblast invasion is considered the ultimate cause of placental related disorders, such as FGR.

\section{Angiogenic markers related to fetal growth restriction}

There are different angiogenic markers associated with defective spiral artery transformation [36]. As the EVT-moves deeper, expression of pregnancy-associated plasma protein-A (PAPP-A) increases. First trimester measurement of this protein in maternal serum may be a useful predictor for FGR [37]. PAPP-A has as main substrate insulin-like growth factor (IGF)-binding proteins 4 and 5. Therefore PAPP-A is a key regulator of IGF bioavailability [38], IGF is thought to play an important role in EVT invasion and hence the early development and vascularization of the placenta and placental bed. Low levels of PAPP-A, resulting in decreased release of IGF and consequently less development and vascularization of placental bed could increase the risk of FGR [39].

Abnormal vascular growth and transformation in the placental bed can create a high vessel resistance network that has been associated with the development of FGR [41]. The vascular endothelial growth factor (VEGF) family plays a major role in regulating vasculogenesis and angiogenesis in early human placentation [42]. Placental growth factor (PIGF) is a pro-angiogenic factor predominantly expressed in placental trophoblast that binds to VEGF-receptor or fms-like tyrosine-1 (sFlt-1), thereby enhancing the pro-angiogenic action of VEGF-A [43]. Besides this membrane-bound receptor form, Flt-1 also exists in a soluble form (sFlt-1), which is secreted by the placenta and scavenges VEGF and PIGF, thereby lowering circulating concentrations of unbound VEGF and PIGF [44]. Reduced placental perfusion and subsequent placental hypoxia are associated with increased production of sFlt-1 and decreased concentrations of PlGF in both human and animal models (Figure 3) [45, 46]. This altered balance in pro-/anti-angiogenic factors is postu- 
lated to cause generalized endothelial dysfunction that is characteristic of FGR and preeclampsia (PE). Therefore, it is conceivable that measuring these placental biomarkers in maternal blood could be used in the prediction of FGR.

The sFlt-1/PIGF ratio had already been used in improving the clinical management for PE. A low sFlt-1/PlGF ratio was highly predictive for ruling out PE and avoiding unnecessary hospitalization with a significant economic impact. Using a cut-off value of 38 , the sFlt-1/PIGF ratio seems to be reliable predictor for the imminent development of PE [47]. Despite many studies linking maternal angiogenic concentrations to $\mathrm{PE}$, there is no systematic review of the predictive performance of PlGF and sFlt-1 for FGR or SGA in first trimester. Even further in second and third trimester, evaluation of the changes in these placental biomarkers during pregnancy could give insight in the developing severity of FGR. Abnormal placental angiogenesis might be reflected in longitudinal biomarker results before biophysical changes on ultrasound can be seen.

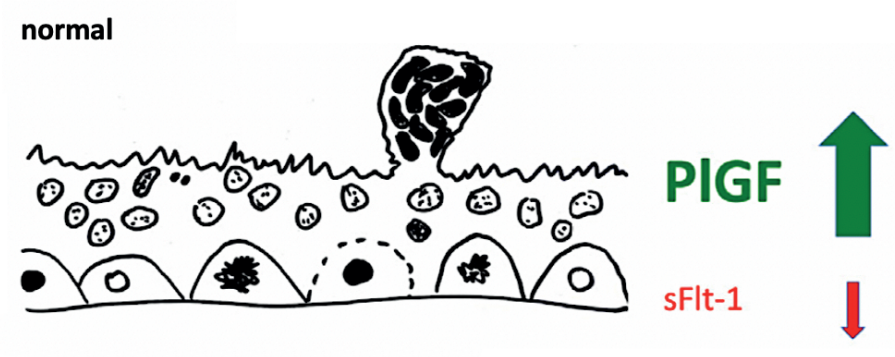

abnormal

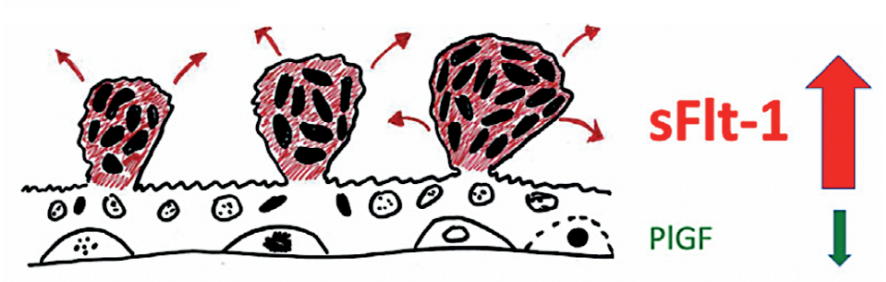

Figure 3 adapted from 'The Placental Basis of Fetal Growth restriction' [40].

Diagrammatic representation of the villous trophoblast surface at the beginning of the third trimester in normal pregnancy and in a pregnancy with MVM of the placenta expressed as earlyonset preeclampsia with fetal growth restriction (FGR) and an elevated maternal serum soluble fms-like tyrosine kinase-1/placental like growth factor (sFlt-1/PlGF) ratio. 


\section{Placental lesions}

The pathophysiologic mechanisms of placental lesions leading to FGR involve reduced invasion into spiral arteries and subsequent failure of spiral artery transformation, which disturbs maternal blood flow into the intervillous space. Subsequently, as spiral arteries remain sensitive to sympathetic stimuli, early and repetitive hypoxic injury events and maternal tissue repair mechanisms may initiate the development of placental defects and maternal vascular malperfusion (MVM) lesions. The Amsterdam Consensus on the definition of various placental characteristics and lesions provide a reliable and extensive framework to study placental abnormalities associated with FGR [48]. These MVM lesions are thought to be the consequence of the process of defective remodeling of the maternal spiral arteries [49-51]. Most MVM lesions are described in pregnancies complicated by SGA. It is yet unclear if these MVM lesions are also seen in placentas from AGA neonates with FGR.

\section{Stages of prediction for fetal growth restriction}

First trimester prediction of FGR may be relevant for timely detection and possible preventive intervention of those at risk [52]. To improve care, there is a need for good prediction models throughout pregnancy. Unfortunately, a lot of prediction models for FGR have poor performance which may partially reflect heterogeneity in definitions, using SGA instead of FGR. The fetal medicine foundation (FMF) developed a prediction model for SGA [53]. We have evaluated this model for term SGA and FGR in our study population.

\section{Postnatal growth pattern}

Fetal growth restriction may affect offspring postnatal growth. Studies indicate that 80 to $85 \%$ of fetuses born SGA increase their height above the lower limit of normal in the first year of life [54, 55]. Usually, offspring born growth restricted, show increased growth velocity in the first 2-3 years, followed by a stable height in childhood, a normal age at onset of puberty, but an adult height below target height [56]. Catch-up growth is considered to be a process of compensatory accelerated growth after a period of poor intra-uterine development [57]. Catch-up growth is called complete when final height is close to the predicted height based on height of both parents. Incomplete catch-up growth associates with increased neurodevelopmental problems in comparison to children with complete catch-up growth [58]. Low birth weight as a consequence of fetal growth restriction is a risk factor for cardiovascular disease later in life [59]. It is still unclear whether this is caused by the catch-up growth itself or the underlying pathophysiology of FGR. Even though a lot of research has been done on postnatal growth and the occur- 
rence of catch-up growth in SGA infants, this phenomenon has not been studied well in AGA infants. Catch-up growth may also appear in AGA infants, especially in AGA infants with reduced growth velocity. These children are currently being considered as completely healthy, based on birth weight, even though this may be incorrect because of their impaired fetal growth trajectory [60]. Proper guidance of those at risk may improve remote health. Detailed coupling of fetal-to-infant growth may be important to understand the importance of catch-up growth. 


\section{AIMS AND OUTLINE OF THIS THESIS}

This thesis is based on seven main research questions

1. Chapter 2: What is the association between reduced fetal growth velocities in AGA neonates and neonatal outcome?

2. Chapter 3: Can we improve the detection of SGA neonates with an adverse neonatal outcome, using fetal growth velocity in combination with maternal placental biomarkers in first trimester?

3. Chapter 4: Is there an additional value of using longitudinal changes in placental biomarkers (PlGF and sFlt-1) in women with early versus late placental dysfunction?

4. Chapter 5: Which cut-off values for the maternal placental biomarkers PIGF and sFlt- 1 can be used for the screening or prediction of SGA neonates based on available literature?

5. Chapter 6: Is there a relationship between maternal vascular malperfusion placental lesions, birthweight percentile and neonatal outcome?

6. Chapter 7: What is the performance of the FMF first trimester risk prediction model for term SGA and reduced growth velocity in our population?

7. Chapter 8: What is the association between fetal growth velocities and postnatal growth during the first 5 years after birth?

In summary, this thesis aims to explore the role of fetal growth velocities and placental biomarkers in the detection of fetal growth restriction and the influence on neonatal outcome.

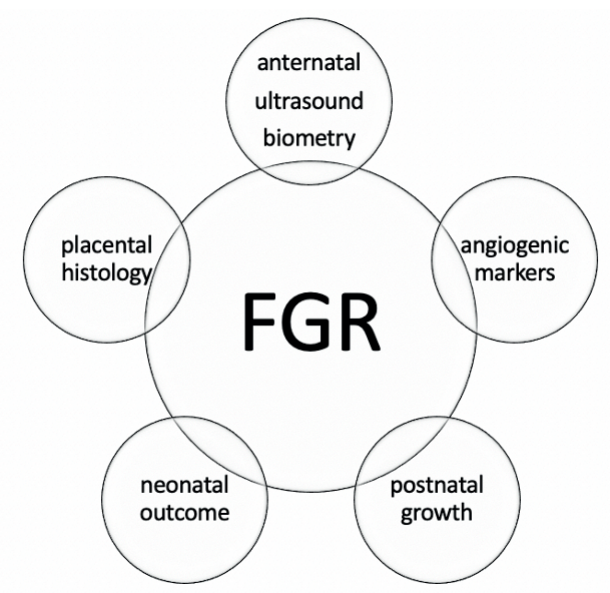

Figure 4 Main topics related to fetal growth restriction in this thesis. 


\section{REFERENCES}

1. Figueras F, Gratacos E. An integrated approach to fetal growth restriction. Best Pract Res Clin Obstet Gynaecol. 2017;38:48-58.

2. Malhotra A, Allison BJ, Castillo-Melendez M, Jenkin G, Polglase GR, Miller SL. Neonatal Morbidities of Fetal Growth Restriction: Pathophysiology and Impact. Front Endocrinol (Lausanne). 2019;10:55.

3. Lees C, Marlow N, Arabin B, Bilardo CM, Brezinka C, Derks JB, et al. Perinatal morbidity and mortality in early-onset fetal growth restriction: cohort outcomes of the trial of randomized umbilical and fetal flow in Europe (TRUFFLE). Ultrasound Obstet Gynecol. 2013;42(4):400-8.

4. Loeffler FE. Clinical foetal weight prediction. J Obstet Gynaecol $\mathrm{Br}$ Commonw. 1967;74(5):675-7.

5. Hall MH, Chng PK, MacGillivray I. Is routine antenatal care worth while? Lancet. 1980;2(8185):78-80.

6. Rosenberg K, Grant JM, Hepburn M. Antenatal detection of growth retardation: actual practice in a large maternity hospital. Br J Obstet Gynaecol. 1982;89(1):12-5.

7. Pay AS, Wiik J, Backe B, Jacobsson B, Strandell A, Klovning A. Symphysis-fundus height measurement to predict small-for-gestational-age status at birth: a systematic review. BMC Pregnancy Childbirth. 2015;15:22.

8. Belizan JM, Villar J, Nardin JC, Malamud J, De Vicurna LS. Diagnosis of intrauterine growth retardation by a simple clinical method: measurement of uterine height. Am J Obstet Gynecol. 1978;131(6):643-6.

9. Hadlock FP, Harrist RB, Sharman RS, Deter RL, Park SK. Estimation of fetal weight with the use of head, body, and femur measurements-a prospective study. Am J Obstet Gynecol. 1985;151(3):333-7.

10. Milner J, Arezina J. The accuracy of ultrasound estimation of fetal weight in comparison to birth weight: A systematic review. Ultrasound. 2018;26(1):32-41.

11. Bamfo JE, Odibo AO. Diagnosis and management of fetal growth restriction. J Pregnancy. 2011;2011:640715.

12. Hiersch L, Melamed N. Fetal growth velocity and body proportion in the assessment of growth. Am J Obstet Gynecol. 2018;218(2S):S700-S11 e1.

13. Cavallaro A, Veglia M, Svirko E, Vannuccini S, Volpe G, Impey L. Using fetal abdominal circumference growth velocity in the prediction of adverse outcome in near-term smallfor-gestational-age fetuses. Ultrasound Obstet Gynecol. 2018;52(4):494-500.

14. Steckel RH. Birth weights and stillbirths in historical perspective. Eur J Clin Nutr. 1998;52 Suppl 1:S16-20.

15. Altman DG, Hytten FE. Intrauterine growth retardation: let's be clear about it. Br J Obstet Gynaecol. 1989;96(10):1127-32.

16. Campbell S, Wilkin D. Ultrasonic measurement of fetal abdomen circumference in the estimation of fetal weight. Br J Obstet Gynaecol. 1975;82(9):689-97.

17. Clausson B, Gardosi J, Francis A, Cnattingius S. Perinatal outcome in SGA births defined by customised versus population-based birthweight standards. BJOG. 2001;108(8):830-4.

18. Lausman A, Kingdom J, Maternal Fetal Medicine C. Intrauterine growth restriction: screening, diagnosis, and management. J Obstet Gynaecol Can. 2013;35(8):741-8. 
19. Lindqvist PG, Molin J. Does antenatal identification of small-for-gestational age fetuses significantly improve their outcome? Ultrasound Obstet Gynecol. 2005;25(3):258-64.

20. Ott WJ. The diagnosis of altered fetal growth. Obstet Gynecol Clin North Am. 1988;15(2):23763.

21. Figueras F, Gardosi J. Intrauterine growth restriction: new concepts in antenatal surveillance, diagnosis, and management. Am J Obstet Gynecol. 2011;204(4):288-300.

22. Gardosi J, Madurasinghe V, Williams M, Malik A, Francis A. Maternal and fetal risk factors for stillbirth: population based study. BMJ. 2013;346:f108.

23. Selvaratnam RJ, Davey MA, Anil S, McDonald SJ, Farrell T, Wallace EM. Does public reporting of the detection of fetal growth restriction improve clinical outcomes: a retrospective cohort study. BJOG. 2020;127(5):581-9.

24. Selvaratnam RJ, Davey MA, Mol BW, Wallace EM. Increasing obstetric intervention for fetal growth restriction is shifting birthweight centiles: a retrospective cohort study. BJOG. 2020.

25. Flenady V, Wojcieszek AM, Middleton P, Ellwood D, Erwich JJ, Coory M, et al. Stillbirths: recall to action in high-income countries. Lancet. 2016;387(10019):691-702.

26. Mifsud W, Sebire NJ. Placental pathology in early-onset and late-onset fetal growth restriction. Fetal Diagn Ther. 2014;36(2):117-28.

27. Heinonen S, Taipale P, Saarikoski S. Weights of placentae from small-for-gestational age infants revisited. Placenta. 2001;22(5):399-404.

28. Hunkapiller NM, Fisher SJ. Chapter 12. Placental remodeling of the uterine vasculature. Methods Enzymol. 2008;445:281-302.

29. Pijnenborg R, Vercruysse L. A.A.W. Hubrecht and the naming of the trophoblast. Placenta. 2013;34(4):314-9.

30. Simpson RA, Mayhew TM, Barnes PR. From 13 weeks to term, the trophoblast of human placenta grows by the continuous recruitment of new proliferative units: a study of nuclear number using the disector. Placenta. 1992;13(5):501-12.

31. Turco MY, Moffett A. Development of the human placenta. Development. 2019;146(22).

32. Pijnenborg R, Dixon G, Robertson WB, Brosens I. Trophoblastic invasion of human decidua from 8 to 18 weeks of pregnancy. Placenta. 1980;1(1):3-19.

33. Pijnenborg R, Vercruysse L, Hanssens M. The uterine spiral arteries in human pregnancy: facts and controversies. Placenta. 2006;27(9-10):939-58.

34. Brosens I, Robertson WB, Dixon HG. The physiological response of the vessels of the placental bed to normal pregnancy. J Pathol Bacteriol. 1967;93(2):569-79.

35. Burton GJ, Jauniaux E, Watson AL. Maternal arterial connections to the placental intervillous space during the first trimester of human pregnancy: the Boyd collection revisited. Am J Obstet Gynecol. 1999;181(3):718-24.

36. Ruchob R, Rutherford JN, Bell AF. A Systematic Review of Placental Biomarkers Predicting Small-for-Gestational-Age Neonates. Biol Res Nurs. 2018;20(3):272-83.

37. Handschuh K, Guibourdenche J, Guesnon M, Laurendeau I, Evain-Brion D, Fournier T. Modulation of PAPP-A expression by PPARgamma in human first trimester trophoblast. Placenta. 2006;27 Suppl A:S127-34.

38. Kalousova M, Muravska A, Zima T. Pregnancy-associated plasma protein A (PAPP-A) and preeclampsia. Adv Clin Chem. 2014;63:169-209.

39. Lau H, Amarasekara C, Uppal T. Low PAPP-A: what are the clinical implications? Australas J Ultrasound Med. 2012;15(1):26-8. 
40. Zur RL, Kingdom JC, Parks WT, Hobson SR. The Placental Basis of Fetal Growth Restriction. Obstet Gynecol Clin North Am. 2020;47(1):81-98.

41. Roberts JM, Escudero C. The placenta in preeclampsia. Pregnancy Hypertens. 2012;2(2):7283.

42. Kato T, Ito Y, Hosono K, Suzuki T, Tamaki H, Minamino T, et al. Vascular endothelial growth factor receptor-1 signaling promotes liver repair through restoration of liver microvasculature after acetaminophen hepatotoxicity. Toxicol Sci. 2011;120(1):218-29.

43. Herraiz I, Quezada MS, Rodriguez-Calvo J, Gomez-Montes E, Villalain C, Galindo A. Longitudinal change of sFlt-1/PlGF ratio in singleton pregnancy with early-onset fetal growth restriction. Ultrasound Obstet Gynecol. 2018;52(5):631-8.

44. Maynard SE, Min JY, Merchan J, Lim KH, Li J, Mondal S, et al. Excess placental soluble fmslike tyrosine kinase 1 (sFlt1) may contribute to endothelial dysfunction, hypertension, and proteinuria in preeclampsia. J Clin Invest. 2003;111(5):649-58.

45. Chau K, Hennessy A, Makris A. Placental growth factor and pre-eclampsia. J Hum Hypertens. 2017;31(12):782-6.

46. Vrachnis N, Kalampokas E, Sifakis S, Vitoratos N, Kalampokas T, Botsis D, et al. Placental growth factor (PIGF): a key to optimizing fetal growth. J Matern Fetal Neonatal Med. 2013;26(10):995-1002.

47. Caillon H, Tardif C, Dumontet E, Winer N, Masson D. Evaluation of sFlt-1/PlGF Ratio for Predicting and Improving Clinical Management of Pre-eclampsia: Experience in a Specialized Perinatal Care Center. Ann Lab Med. 2018;38(2):95-101.

48. Khong TY, Mooney EE, Ariel I, Balmus NC, Boyd TK, Brundler MA, et al. Sampling and Definitions of Placental Lesions: Amsterdam Placental Workshop Group Consensus Statement. Arch Pathol Lab Med. 2016;140(7):698-713.

49. Gibbs I, Leavey K, Benton SJ, Grynspan D, Bainbridge SA, Cox BJ. Placental transcriptional and histologic subtypes of normotensive fetal growth restriction are comparable to preeclampsia. Am J Obstet Gynecol. 2019;220(1):110 e1- e21.

50. Benton SJ, McCowan LM, Heazell AE, Grynspan D, Hutcheon JA, Senger C, et al. Placental growth factor as a marker of fetal growth restriction caused by placental dysfunction. Placenta. 2016;42:1-8.

51. Whitten AE, Romero R, Korzeniewski SJ, Tarca AL, Schwartz AG, Yeo L, et al. Evidence of an imbalance of angiogenic/antiangiogenic factors in massive perivillous fibrin deposition (maternal floor infarction): a placental lesion associated with recurrent miscarriage and fetal death. Am J Obstet Gynecol. 2013;208(4):310 e1- e11.

52. Bujold E, Roberge S, Lacasse Y, Bureau M, Audibert F, Marcoux S, et al. Prevention of preeclampsia and intrauterine growth restriction with aspirin started in early pregnancy: a meta-analysis. Obstet Gynecol. 2010;116(2 Pt 1):402-14.

53. Poon LC, Syngelaki A, Akolekar R, Lai J, Nicolaides KH. Combined screening for preeclampsia and small for gestational age at 11-13 weeks. Fetal Diagn Ther. 2013;33(1):16-27.

54. Hokken-Koelega AC, De Ridder MA, Lemmen RJ, Den Hartog H, De Muinck KeizerSchrama SM, Drop SL. Children born small for gestational age: do they catch up? Pediatr Res. 1995;38(2):267-71.

55. Albertsson-Wikland K, Wennergren G, Wennergren M, Vilbergsson G, Rosberg S. Longitudinal follow-up of growth in children born small for gestational age. Acta Paediatr. 1993;82(5):438-43. 
56. Albertsson-Wikland K, Karlberg J. Natural growth in children born SGA with and without catch up growth. Horm Res. 2003;59 Suppl 1:129.

57. Claris O, Beltrand J, Levy-Marchal C. Consequences of intrauterine growth and early neonatal catch-up growth. Semin Perinatol. 2010;34(3):207-10.

58. Wit JM, Boersma B. Catch-up growth: definition, mechanisms, and models. J Pediatr Endocrinol Metab. 2002;15 Suppl 5:1229-41.

59. de Boo HA, Harding JE. The developmental origins of adult disease (Barker) hypothesis. Aust N Z J Obstet Gynaecol. 2006;46(1):4-14.

60. Broere-Brown ZA, Schalekamp-Timmermans S, Jaddoe VWV, Steegers EAP. Deceleration of fetal growth rate as alternative predictor for childhood outcomes: a birth cohort study. BMC Pregnancy Childbirth. 2019;19(1):216. 


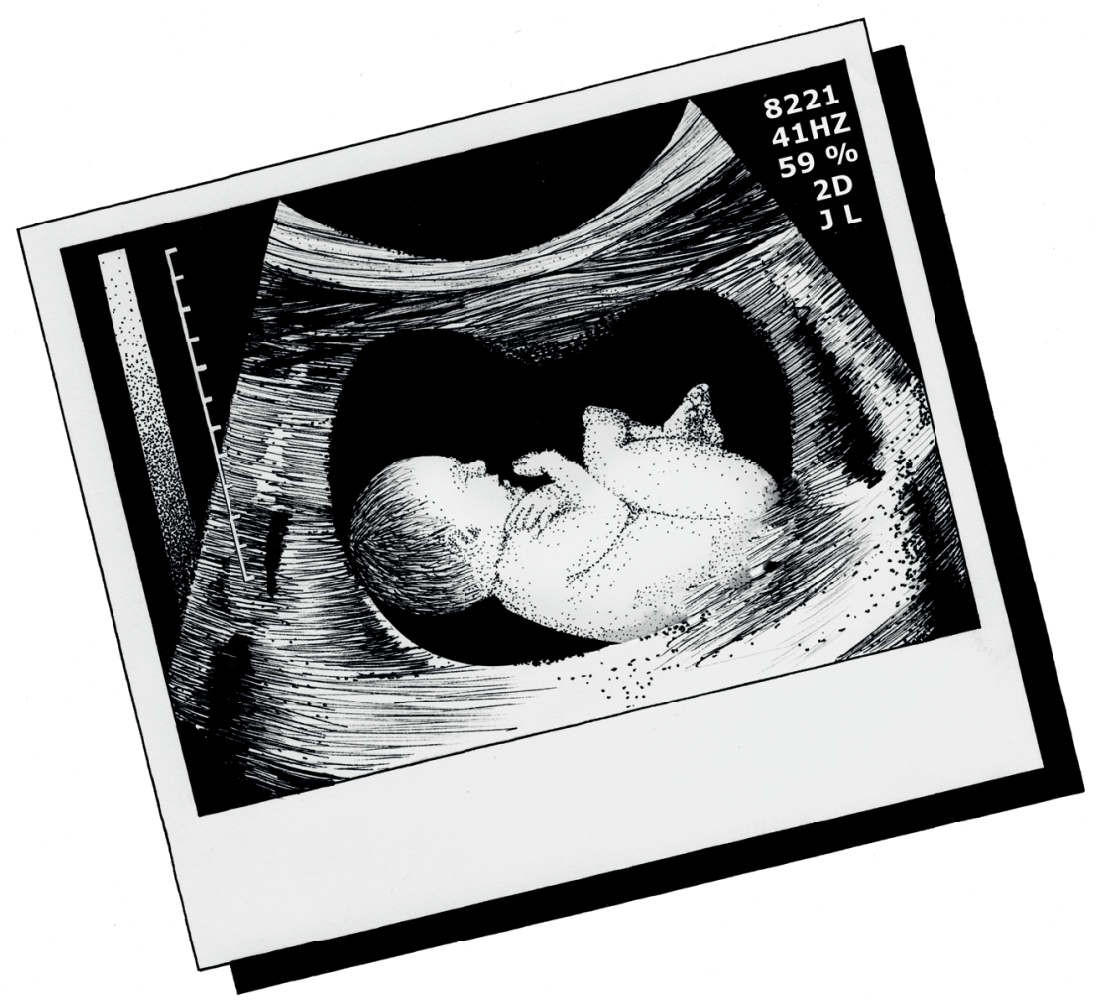




\section{Reduced fetal growth velocities and the association with neonatal outcomes in appropriate-for- gestational-age neonates: a retrospective cohort study}

MLE Hendrix

SMJ van Kuijk

AWD Gavilanes

D Kramer

MEA Spaanderman

S Al Nasiry

BMC Pregnancy Childbirth. 2019; 19(1):31. 


\section{ABSTRACT}

\section{Background}

Fetal growth restriction is, despite advances in neonatal care and uptake of antenatal ultrasound scanning, still a major cause of perinatal morbidity. Neonates with birth weight $>10^{\text {th }}$ percentile are assumed to be appropriate-for-gestationalage (AGA), although many are at increased risk of perinatal morbidity, because of undetected mild restriction of growth potential. We hypothesized that within AGA neonates, reduced fetal growth velocities are associated with adverse neonatal outcome.

\section{Methods}

A retrospective cohort study of singleton pregnancies, in the Maastricht University Medical Centre (MUMC) between 2010 and 2016. Women had two fetal biometry scans (18-22 weeks and 30-34 weeks of gestational age) and delivered a newborn with a birth weight between the 10 th-80th percentile.

Differences in growth velocities of the abdominal circumference (AC), biparietal diameter (BPD), head circumference (HC) and femur length (FL) were compared between the suboptimal AGA (sAGA) (birth weight centiles 10-50) and optimal AGA (oAGA) (birth weight centiles 50-80) group. We assessed the association between velocities and neonatal outcomes.

\section{Results}

We included 934 singleton pregnancies. In the suboptimal AGA group, fetal growth velocities were lower (in mm/week): AC $10.72 \pm 1.00$ vs $11.23 \pm 1.00$ ( $\mathrm{p}<.001$ ), HC $10.50 \pm 0.80$ vs $10.68 \pm 0.77$ ( $\mathrm{p}=0.001)$, BPD $3.01 \pm 0.28$ vs $3.08 \pm 0.27(\mathrm{p}<.0001)$ and FL $2.47 \pm 0.21$ vs $2.50 \pm 0.22$ ( $\mathrm{p}=0.014$ ), compared to the optimal AGA group. Neonates with an adverse neonatal outcome had significantly lower growth velocities (in mm/week) of: AC 10.57 vs 10.94 ( $\mathrm{p}=0.034)$, HC 10.28 vs 10.59 ( $\mathrm{p}=0.003$ ) and BPD 2.97 vs 3.04 ( $\mathrm{p}=0.043$ ) compared to those with normal outcome. An inverse association was observed between the $\mathrm{AC}$ velocity and a composite adverse neonatal outcome $(\mathrm{OR})=0.667(95 \% \mathrm{CI}$ 0.507-0.879, $\mathrm{p}=0.004)$, and between the AC velocity and neonates with NICU stay $(\mathrm{OR})=0.733$ (95\%CI 0.570-0.942, $\mathrm{p}=0.015)$. Neonates with a birthweight lower than expected (based on the abdominal circumference at 20 weeks) had significantly more composite adverse neonatal outcomes $8.5 \%$ vs $5.0 \%(\mathrm{p}=0.047)$, NICU stays $9.6 \%$ vs $3.8 \%(\mathrm{p}<.0001)$ and hospital stays $44.4 \%$ vs $35.6 \%(\mathrm{p}=0.006)$. 


\section{Conclusion}

Appropriate-for-gestational-age neonates are a heterogeneous group with some showing suboptimal fetal growth. Abnormal fetal growth velocities, especially abdominal circumference velocity, are associated with adverse neonatal outcome and can potentially improve the detection of mild growth restriction when used in multivariate models.

\section{KEYWORDS}

Fetal growth restriction, abdominal circumference velocity, neonatal outcome, ultrasound, appropriate-for-gestational-age neonates

\section{KEY MESSAGE}

There is a subgroup within appropriate-for-gestational age neonates, who are at increased risk of perinatal morbidity. The abdominal circumference velocity could be a detector for this group with mild growth restriction. 


\section{BACKGROUND}

Fetal growth restriction (FGR) is one of the most frequently encountered problems in modern obstetrics, with a major impact on perinatal mortality and morbidity [1]. FGR is a controversial and complex entity, due to its multifactorial aetiology and the unclear link between its pathophysiology and its current definitions.

Literature has often interchangeably used the terms 'intrauterine growth retardation', 'intrauterine growth restriction' (both given the acronym IUGR) and fetal growth restriction (FGR), to indicate abnormally small fetus suspected antenatally, based on ultrasound parameters of fetal growth, most commonly expected fetal weight (EFW) and abdominal circumference (AC) [2]. We prefer to use the moderner term FGR being more specific, i.e. pertaining to the real problem, the fetus, and not to other intrauterine tissues, and additionally, not having the negative connotation of the word "retardation". Traditionally, EFW and $\mathrm{AC}$ are plotted against population curves, on which there is no consensus and are the subject of ongoing debate, and IUGR is most commonly defined as EFW of AC values below the $3^{\text {rd }}, 5^{\text {th }}$ or $10^{\text {th }}$ centile [3]. Small for gestational age (SGA) is the term used postnatally to describe a newborn with abnormally low birth weight for a specific gestational period, using variable cut-off points in literature: $3^{\text {rd }}$, $5^{\text {th }}$ or $10^{\text {th }}$ centile. The term SGA has also been inappropriately used antenatelly to define fetus with mild to moderate "smalleness" (EFW or AC between $3^{\text {rd }}-10^{\text {th }}$ centile.

These definitions are based on cut off points and statistical assumptions derived from epidemiological data, rather than being derived from physiopathological mechanisms. It is conceivable that the mechanism restricting fetal growth can have variable severity, leading in mild cases to a decrease in growth velocity but not enough for birth weight to drop below the $10^{\text {th }}$ centile. Hence, these neonates with restricted growth potential are falsely labelled as appropriate-for-gestational age (AGA) according to many commonly used population charts.

Unlike severe early FGR, mild and late onset FGR in the AGA group is difficult to diagnose and more elusive to currently used predictive tests $[4,5]$. Fetuses with the lowest birth weight centiles have a significantly higher risk of perinatal mortality compared to the $50-90^{\text {th }}$ centile [6]. This largely undetected type of growth restriction is responsible for a significant percentage of stillbirths and neonates with adverse neonatal outcome $[7,8]$. Routine third trimester biometry scans could improve the detection of FGR in the AGA group [9]. However, such scans take a 'snapshot' approach to fetal growth and fail to take into account biological variation influenced by the genetic background and also the concept of supoptimal fetal growth being a dynamic process. A better approach is to con- 
sider the difference in a growth parameter between two antenatal time points, corrected for the genetically determinded growth potential of an individial fetus, tentatively expressed as 'growth velocity' [10]. This, individualised growth potential can describe a type of growth restriction characterized by a drop in growth potential without necessarily being overtly small [11]. We hypothesized that within the AGA group, reduced fetal growth velocities between 20 and 32 weeks of gestation is associated with lower birth weight percentiles and an increased risk of an adverse neonatal outcome.

\section{METHODS}

\section{Study design and participants}

In this retrospective cohort study, we combined data from two electronic patient databases: the antenatal ultrasound database and the labour ward database of the Maastricht University Medical Centre (MUMC) to make an integrated database. We included women with singleton pregnancies without congenital anomalies, who delivered between April 2010 and July 2016 in the Maastricht University Medical Centre, and who had two available fetal growth scans, one between 18 and 22 weeks of gestational age and one between 30 and 34 weeks of gestational age. The pregnancies were dated according to the formula of Robinson calculated from crown-rump length (CRL) measurement in the first trimester [12]. We only included women, who had a delivery of a fetus with a birth weight between the 10th-80th centile. We excluded women with a birth weight below the $10^{\text {th }}$ centile (SGA) and above the $80^{\text {th }}$ centile (large-for gestational age). Patients were informed about research in this university medical centre and were given information about the data collection from their records for research purpose. Patients who refuse using their records, were excluded in this study. The study protocol was approved by the medical ethical committee of the Maastricht University Medical Centre (17-4-0.15.1/ab). All procedures were in accordance with institutional guidelines and adhered to the principles of the Declaration of Helsinki and Title 45, U.S. Code of Federal Regulations, Part 46, Protection of Human Subjects (revised 13 November 2001, effective 13 December 2001).

\section{Ultrasound growth examination}

At the first hospital intake, data on length, weight, smoking status and medical and obstetric history, were collected and recorded in an electronic medical file. Body mass index was calculated by weight $(\mathrm{kg})$ divided by height $(\mathrm{m})$-squared. An experienced sonographer recorded ultrasound measurements of abdominal cir- 
cumference (AC), biparietal diameter (BPD), head circumference (HC) and femur length (FL), using a GE Voluson ultrasound machine, with a 2-5MHz curved-array transducer, in accordance with the routine mid-trimester fetal ultrasound scan guidelines [13]. These fetal growth scans were performed in two periods, around 20wks (18-22 weeks) during the second trimester anomaly scan, and a second around 32 weeks (30-34 weeks) as a routine third trimester growth scan [14, 15]. Fetal growth parameters were recorded in Astraia electronic fetal medicine database (Astraia GMBH; Munich, Germany). Estimated fetal weight was calculated using the Hadlock equation[16] [Hadlock C; $\log (10)$ BW $=1.335$ - 0.0034(abdominal circumference $[\mathrm{AC}])($ femur length $[\mathrm{FL}])+0.0316$ (biparietal diameter) $+0.0457(\mathrm{AC})$ $+0.1623(\mathrm{FL})$. For the purpose of this article we calculated velocities of each of the fetal growth parameters (in $\mathrm{mm} /$ week) as the difference in actual values (rather than percentiles) of the measured parameters between the two examination periods, divided by the number of weeks. Birth weight (core outcome) was transformed to percentiles according to the Dutch reference standard [17] with a birth weight between the $10^{\text {th }}$ and $80^{\text {th }}$ percentile considered to be appropriatefor-gestational-age (AGA). Further this AGA group was divided into two categories: 1) birth weight centiles $10-50$ as the suboptimal appropriate-for-gestational-age (sAGA) group and 2) birth weight centiles 50-80 as the optimal appropriate-forgestational-age group (oAGA).

In addition, we compared neonatal outcomes in two groups of neonates based on the premise that a fetus with an above average abdominal circumference at 20 weeks of gestation is "expected" to have an above average birth weight, and vice versa. Consequently, the first group consisted of neonates who had a below average birth weight despite an above average AC at 20 weeks (birth weight < expected), suggesting a late reduction in growth velocity, and the second group consisted of neonates who had a higher than average birth weight despite a below average AC at 20 weeks (birth weight $\geq$ expected).

\section{Neonatal data}

Delivery and neonatal outcomes were registered by the obstetrician or in case of an adverse outcome by the paediatrician. Data about induction of labour and mode of delivery were collected. Mode of delivery was divided in subcategories; spontaneous vaginal, assisted vaginal, intrapartum- or prelabour caesarean. All deliveries were in the same hospital.

APGAR-scores were assessed at 1 and 5 min after birth. We used a cut-off of less than 5 at $5 \mathrm{~min}$ to determine adverse outcome [18]. Metabolic acidosis was defined as an umbilical artery blood $\mathrm{pH}<7.0$ and base deficit $>12 \mathrm{mmol} / \mathrm{L}$ ) [19]. The composite adverse neonatal outcome consisted of 4 complications: asphyxia, 
sepsis, respiratory distress syndrome [20] and transient tachypnea of the newborn [21]. These complications were recorded in medical files and were defined according to the judgments of the attending medical staff. Asphyxia was defined as intrapartum-related hypoxia-ischemia with multiple organ failure including encephalopathy [22]. Neonatal stay at the hospital was divided in general hospital stay and neonatal intensive care unit admission.

\section{Statistical analysis}

The general characteristics were presented as mean and standard deviation (SD) for the total cohort and stratified by birth weight percentiles (50-80 as optimal appropriate-for-gestational-age (oAGA) group), and the birth weight percentiles 10-50 as suboptimal appropriate-for-gestational-age (sAGA) group).

Differences between the groups were calculated with the independent T-test for continuous variables and the Chi-square test for categorical variables. Analysis of variance (ANOVA) was used to compare growth velocities between different groups of birth weight percentiles. Post-hoc testing of differences was corrected for multiple testing using the Bonferroni-method. Growth velocities we reported using mean, SD and range. We used logistic regression analysis to estimate the association between abdominal circumference velocity and dichotomous neonatal outcomes, corrected for potential confounders. We considered maternal age, BMI and parity as potential confounding variables. All analyses were performed using SPSS Statistics 23 (IBM Corp, Armonk, NY). P-values $<0.05$ were considered to indicate statistical significance.

\section{RESULTS}

\section{Participants}

Between April 2010 and July 2016, we identified 7.720 pregnant women, with 28.649 fetal biometry scans. We excluded scans $<18$ weeks of gestational age $(n=2.762)$, between $22-30$ weeks of gestational age $(n=5.641)$ and $>34$ weeks of gestational age $(n=3.789)$. After exclusion of twin pregnancies $(n=362)$, we had 7.348 singleton pregnancies in the labour ward database and 16.457 fetal biometry scans between 18-22 and 30-34 weeks of gestational age in the antenatal ultrasound database.

After cross-matching the two databases, women with growth scans outside the range of the intended periods or who delivered outside the MUMC hospital were excluded. Also, we excluded neonates with a birth weight percentile $<10$ ( $n=953)$ 
or birth weight percentile $>80$ ( $\mathrm{n}=213)$, as well as those with intra-uterine fetal death $(\mathrm{n}=18)$ and congenital anomalies (within 4 neonatal death) $(\mathrm{n}=116)$

The final study cohort consisted of 934 singleton pregnancies (birth weight centiles 10-80) and 1.868 fetal ultrasound biometry scans (Figure 1). The neonates were divided in categories: birth weight percentiles 10-50, considered as suboptimal appropriate-for-gestational-age (sAGA) $(\mathrm{n}=569)$ and birth weight percentile 50-80, considered as optimal appropriate-for-gestational-age (oAGA) $(n=365)$.

The baseline characteristics of the study population are given in Table 1 . Women had a mean age of $31.6 \pm 5.1$ years and a mean body mass index of $25.0 \pm 5.7 \mathrm{~kg} /$ $\mathrm{m} 2,44.4 \%$ were primiparous and $11.8 \%$ were smokers. There were no statistically significant differences between the optimal and suboptimal AGA groups in baseline characteristics, and in gestational age at birth, 271.0 13.7 (212-295) days vs. $271.8 \pm 12.2(215-295)$ days ( $\mathrm{p}=0.35)$, except a lower percentage of ovulation induction $(2.5 \%$ vs $0.7 \%)(\mathrm{p}=0.025)$. There was an obvious difference in birth weight (g) between the groups: $3425.4 \pm 421.6(1440-4125)$ in the optimal AGA vs. 3041.5 \pm $402.3(1353-3818)$ in the suboptimal AGA group $(\mathrm{p}<0.001)$.

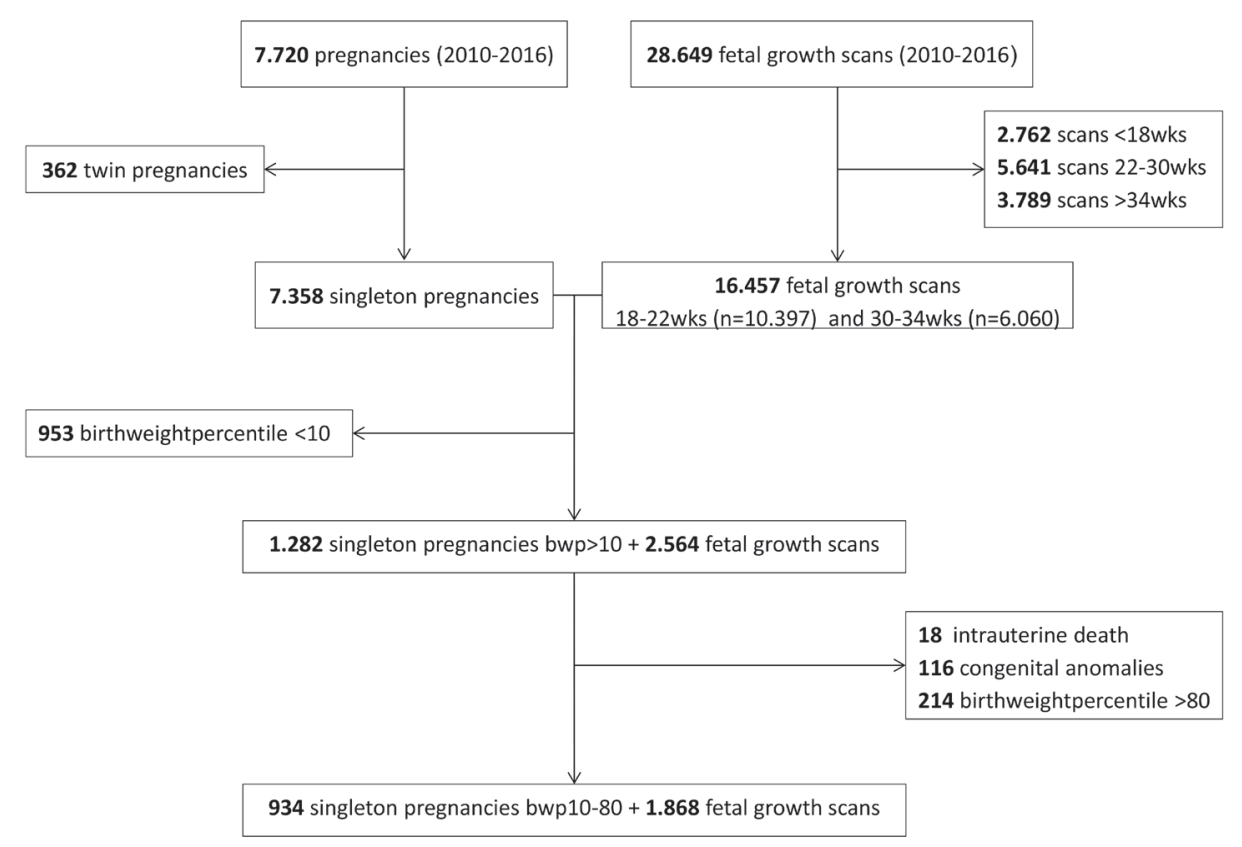

Figure 1 Flow chart of matched pregnancies and fetal growth scans in the appropriate-for-gestational age (AGA) group. 
Table 1. Patient general characteristics of total study cohort with delivery details and stratified according to birth weight percentile category in oAGA (reference group) and sAGA group.

\begin{tabular}{|c|c|c|c|c|}
\hline & $\begin{array}{l}\text { Total cohort AGA } \\
\qquad(\mathrm{n}=934)\end{array}$ & $\begin{array}{c}\text { Optimal AGA } \\
\text { (oAGA) Bwp50-80 } \\
(\mathrm{n}=365)\end{array}$ & $\begin{array}{l}\text { Suboptimal AGA } \\
\text { (sAGA) Bwp10-50 } \\
(\mathrm{n}=569)\end{array}$ & P-value \\
\hline Maternal age (years) & $31.6 \pm 5.1(17-55)$ & $31.6 \pm 5.1(17-55)$ & $31.5 \pm 5.1(17-45)$ & 0.816 \\
\hline Primiparous (\%) & $412(44.4 \%)$ & $160(44.2 \%)$ & 252 (44.6\%) & 0.904 \\
\hline Smoking (\%) & 89 (11.8\%) & 27 (9.2\%) & $62(13.6 \%)$ & 0.067 \\
\hline Height (m) & $1.67 \pm 0.07(1.45-1.86)$ & $1.68 \pm 0.07(1.49-1.86)$ & $1.67 \pm 0.07(1.45-1.86)$ & 0.362 \\
\hline BMI $\left(\mathrm{kg} / \mathrm{m}^{2}\right)$ & $25.0 \pm 5.7(15.7-48.5)$ & $25.5 \pm 5.5(16.7-48.3)$ & $24.7 \pm 5.9(15.7-48.5)$ & 0.164 \\
\hline \multicolumn{5}{|l|}{ Mode of conception } \\
\hline Spontaneous & 775 (91.7\%) & 335 (91.8\%) & 529 (93.0\%) & 0.501 \\
\hline IUI & $13(1.5 \%)$ & $4(1.1 \%)$ & $9(1.6 \%)$ & 0.536 \\
\hline ICSI & $25(3.0 \%)$ & $8(2.2 \%)$ & $17(3.0 \%)$ & 0.462 \\
\hline IVF & $19(2.2 \%)$ & $9(2.5 \%)$ & $10(1.8 \%)$ & 0.454 \\
\hline Ovulation induction & $13(1.5 \%)$ & $9(2.5 \%)$ & $4(0.7 \%)$ & 0.025 \\
\hline GA at birth (days) & $271.5 \pm 12.8(212-295)$ & $271.0 \pm 13.7(212-295)$ & $271.8 \pm 12.2(215-295)$ & 0.350 \\
\hline GA <34wks (n) & $23(2.5 \%)$ & $13(3.6 \%)$ & $10(1.8 \%)$ & 0.088 \\
\hline GA 34-36wks (n) & $40(4.3 \%)$ & 15 (4.1\%) & 25 (4.4\%) & 0.870 \\
\hline GA > 36wks (n) & 871 (93.3\%) & 337 (92.3\%) & 534 (93.8\%) & 0.423 \\
\hline Birth weight (g) & $\begin{array}{c}3191.55 \pm 450.6 \\
(1353-4125)\end{array}$ & $\begin{array}{c}3425.4 \pm 421.6 \\
1440-4125)\end{array}$ & $\begin{array}{c}3041.5 \pm 402.3 \\
(1353-3818)\end{array}$ & $<0.001$ \\
\hline Induction of labour(yes) & 205 (21.9\%) & 89 (24.4\%) & 116 (20.3\%) & $<0.001$ \\
\hline \multicolumn{5}{|l|}{ Mode of delivery } \\
\hline Spontaneous vaginal & $722(77.3 \%)$ & $278(76.2 \%)$ & 444 (78.0\%) & 0.522 \\
\hline Assisted vaginal & $124(13.3 \%)$ & 47 (12.9\%) & 77 (13.5\%) & 0.843 \\
\hline Intrapartum caesarean & $32(3.4 \%)$ & 17 (3.0\%) & 15 (4.1\%) & 0.363 \\
\hline Prelabour caesarean & $56(6.0 \%)$ & $25(6.8 \%)$ & $31(5.4 \%)$ & 0.399 \\
\hline
\end{tabular}

Data are expressed as mean \pm standard deviation (min-max) or $n$ (\%). BMI, body mass index; GA, gestational age.

\section{Fetal growth velocities}

Compared to oAGA neonates (BW p50-80), the sAGA group (BW p10-50) had reduced growth velocities across all studied parameters (in $\mathrm{mm} /$ week) (Table 2): abdominal circumference ( $10.72 \pm 1.00$ vs $11.23 \pm 1.00, \mathrm{p}<.001)$, head circumference (10.50 \pm 0.80 vs $10.68 \pm 0.77, \mathrm{p}=0.001)$, biparietal diameter $(3.01 \pm 0.28$ vs $3.08 \pm 0.27$, $\mathrm{p}<.0001)$ and femur length $(2.47 \pm 0.21$ vs $2.50 \pm 0.22, \mathrm{p}=0.014)$, respectively. Within the sAGA neonates, the difference in growth velocity (in $\mathrm{mm} /$ week) was most prominent among the subgroup with the lowest birth weight percentiles (i.e. BW p10-16) for AC (10.45 $\pm 0.96, \mathrm{p}<.0001), \mathrm{HC}(10.26, \mathrm{p}<.001)$ and $\operatorname{BPD}(2.91, \mathrm{p}<.001)$ compared to the oAGA reference group (Figure 2, only data for AC are shown). There was a linear relationship between AC velocity and birth weight (in gram, $\mathrm{mm} /$ week) (Birth weight=1951.67+113.57 x AC velocity, $\mathrm{p}<.0001, \mathrm{R}^{2}=0.067$ ). 
Table 2. Ultrasound growth velocities (20-32 weeks, in mm/week) in the optimal appropriate-forgestational age (oAGA) and suboptimal appropriate-for-gestational age group (sAGA).

\begin{tabular}{lccc}
\hline & $\begin{array}{c}\text { Optimal AGA (oAGA) } \\
\text { group }(\mathbf{n = 3 6 5 )} \\
\text { Bwp50-80 }\end{array}$ & $\begin{array}{c}\text { Suboptimal AGA (sAGA) } \\
\text { group (n=569) } \\
\text { Bwp10-50 }\end{array}$ & P-value \\
\hline Abdominal circumference velocity & $11.23 \pm 1.00(8.19-14.28)$ & $10.72 \pm 1.00(7.70-14.00)$ & $<.0001$ \\
\hline Head circumference velocity & $10.68 \pm 0.77(8.54-13.51)$ & $10.50 \pm 0.80(6.79-12.67)$ & 0.001 \\
\hline Biparietal diameter velocity & $3.08 \pm 0.27(2.31-3.99)$ & $3.01 \pm 0.28(1.96-3.85)$ & $<.0001$ \\
\hline Femur length velocity & $2.50 \pm 0.22(1.89-3.01)$ & $2.47 \pm 0.21(1.54-3.08)$ & 0.014 \\
\hline
\end{tabular}

Data are expressed as mean \pm standard deviation (min-max).

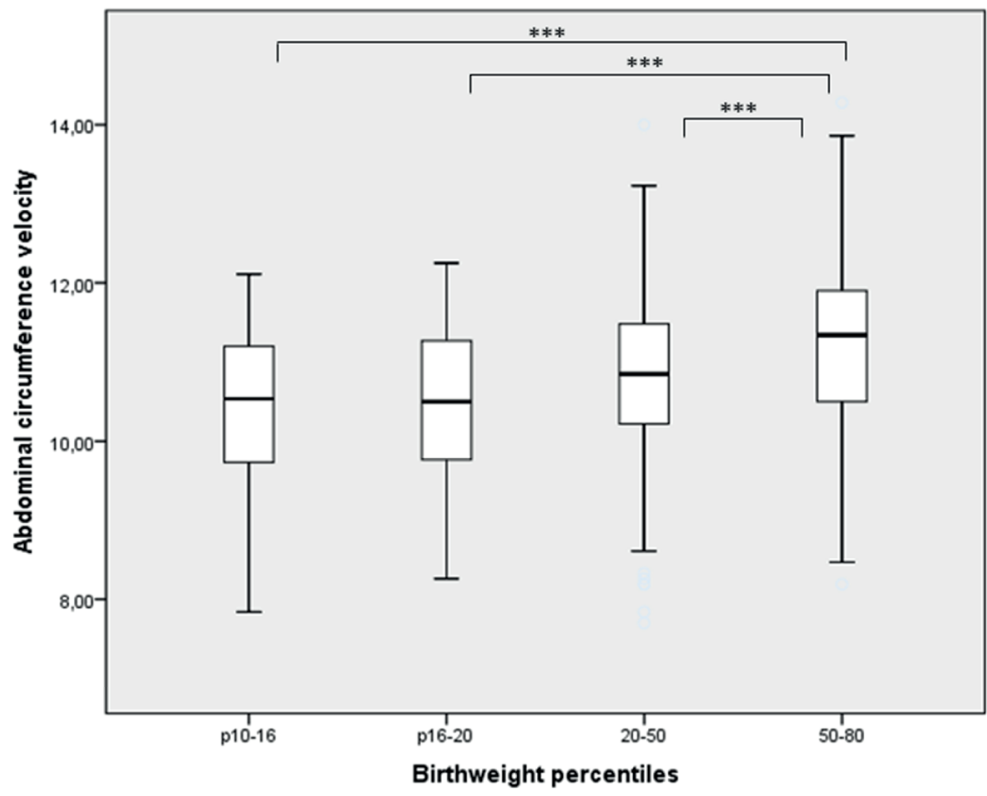

Figure 2 Boxplot of abdominal circumference velocity ( $\mathrm{mm} /$ week) in the optimal appropriatefor-gestational age group (oAGA) (birthweight percentile 50-80) and the birthweight percentile 10-16, 16-20 and 20-50.

$\mathrm{P}$-values, ${ }^{*}=\mathrm{P}<0.05,{ }^{* *} \mathrm{P}<0.01,{ }^{* * *} \mathrm{P}<0.001$

\section{Neonatal outcomes}

There were neither differences between the sAGA and oAGA in the frequency of induction of labour and the mode of delivery, nor in neonatal outcomes: composite adverse outcome, NICU and hospital stays, metabolic acidosis and APGAR at 5 minutes $<5$ (Table 3). However, using this composite adverse neonatal outcome as the independent variable to divide the cohort, neonates with an adverse composite outcome had significantly lower growth velocities (in $\mathrm{mm} / \mathrm{week}$ ) in abdominal circumference: $10.57 \pm 1.33$ vs $10.94 \pm 1.00$ ( $\mathrm{p}=0.034$ ), head circumference 
$10.28 \pm 0.99$ vs $10.59 \pm 0.79(\mathrm{p}=0.003)$ and biparietal diameter $2.97 \pm 0.30$ vs $3.04 \pm 0.27$ $(\mathrm{p}=0.043)$ compared to neonates with normal outcome (Table 4).

Table 3. Neonatal outcomes in the optimal appropriate-for-gestational age (oAGA) and suboptimal appropriate-for-gestational age (sAGA) group.

\begin{tabular}{lccc}
\hline & $\begin{array}{c}\text { Optimal AGA (oAGA) } \\
\text { group (n=365) } \\
\text { Bwp50-80 }\end{array}$ & $\begin{array}{c}\text { Suboptimal AGA (sAGA) } \\
\text { group (n=569) } \\
\text { Bwp10-50 }\end{array}$ & P-value \\
\hline Composite adverse neonatal outcome & $25(6.8 \%)$ & $37(6.5 \%)$ & 0.919 \\
\hline Hypoglycemia & $23(6.3 \%)$ & $52(9.1 \%)$ & 0.139 \\
\hline NICU stay (yes/no) & $26(7.1 \%)$ & $35(6.2 \%)$ & 0.588 \\
\hline Hospital stay (yes/no) & $145(39.7 \%)$ & $226(39.7 \%)$ & 0.998 \\
\hline Metabolic acidosis & $14(5.2 \%)$ & $29(6.5 \%)$ & 0.520 \\
\hline APGAR 5 min $<=5$ & $5(1.4 \%)$ & $6(1.1 \%)$ & 0.759 \\
\hline APGAR 5 min $<=3$ & $0(0 \%)$ & $2(0.6 \%)$ & 0.523 \\
\hline
\end{tabular}

Data are expressed as $n$ (\%). Composite adverse neonatal outcome: asphyxia, sepsis, respiratory distress syndrome and transient tachypnoea of the newborn; NICU, neonatal intensive care unit. Metabolic acidosis, blood $\mathrm{pH}<7.0$ and base deficit $>12 \mathrm{mmol} / \mathrm{L}$.

Table 4. Ultrasound growth velocities ( $\mathrm{mm} /$ week) in adverse and healthy neonatal outcome.

\begin{tabular}{lccc}
\hline & $\begin{array}{c}\text { Composite adverse } \\
\text { neonatal outcome } \\
(\mathbf{n = 6 2 )}\end{array}$ & $\begin{array}{c}\text { Healthy outcome } \\
(\mathbf{n = 8 7 2})\end{array}$ & P-value \\
\hline Abdominal circumference velocity & $10.57 \pm 1.33(7.70-13.86)$ & $10.94 \pm 1.00(7.84-14.28)$ & 0.034 \\
\hline Head circumference velocity & $10.28 \pm 0.99(6.79-13.51)$ & $10.59 \pm 0.79(8.26-13.51)$ & 0.003 \\
\hline Biparietal diameter velocity & $2.97 \pm 0.30(1.96-3.99)$ & $3.04 \pm 0.27(2.24-3.99)$ & 0.043 \\
\hline Femur length velocity & $2.46 \pm 0.27(1.54-3.08)$ & $2.48 \pm 0.21(1.75-3.08)$ & 0.579 \\
\hline
\end{tabular}

Data are expressed as mean \pm standard deviation (min-max)

There was an inverse association between the abdominal circumference velocity and a composite adverse neonatal outcome (-odds ratio $(\mathrm{OR})=0.667$ (95\%CI 0.507-0.879, $\mathrm{p}=0.004)$, and between the abdominal circumference velocity and a neonate with NICU stay, (OR=0.733 (95\% CI 0.570-0.942, $\mathrm{p}=0.015)$. There was no significant association between the abdominal circumference velocity and neonatal hospital stay, $(\mathrm{OR}=0.915$ (95\%CI 0.805-1.039, $\mathrm{p}=0.171)$, metabolic acidosis, $(\mathrm{OR}=1.144$ (95\%CI 0.850-1.541, p=0.374) and APGAR at $5 \mathrm{~min}<5,(\mathrm{OR}=1.102$ (95\%CI 0.558-2.174, $\mathrm{p}=0.780$ ). All associations were corrected for maternal age, BMI and parity.

In addition, neonates who had a below average birth weight despite an above average $\mathrm{AC}$ at 20 weeks (birth weight < expected), suggesting a late reduction in growth velocity had significantly more composite adverse neonatal outcomes 
(8.5\% vs 5.0\%, $\mathrm{p}=0.047)$, more NICU stays $(9.6 \%$ vs $3.8 \%, \mathrm{p}=<.0001)$ and more hospital admissions ( $44.4 \%$ vs $35.6 \%, \mathrm{p}=0.006$ ), compared to neonates who had a higher than average birth weight despite a below average AC at 20 weeks (birth weight $\geq$ expected) (Figure 3).

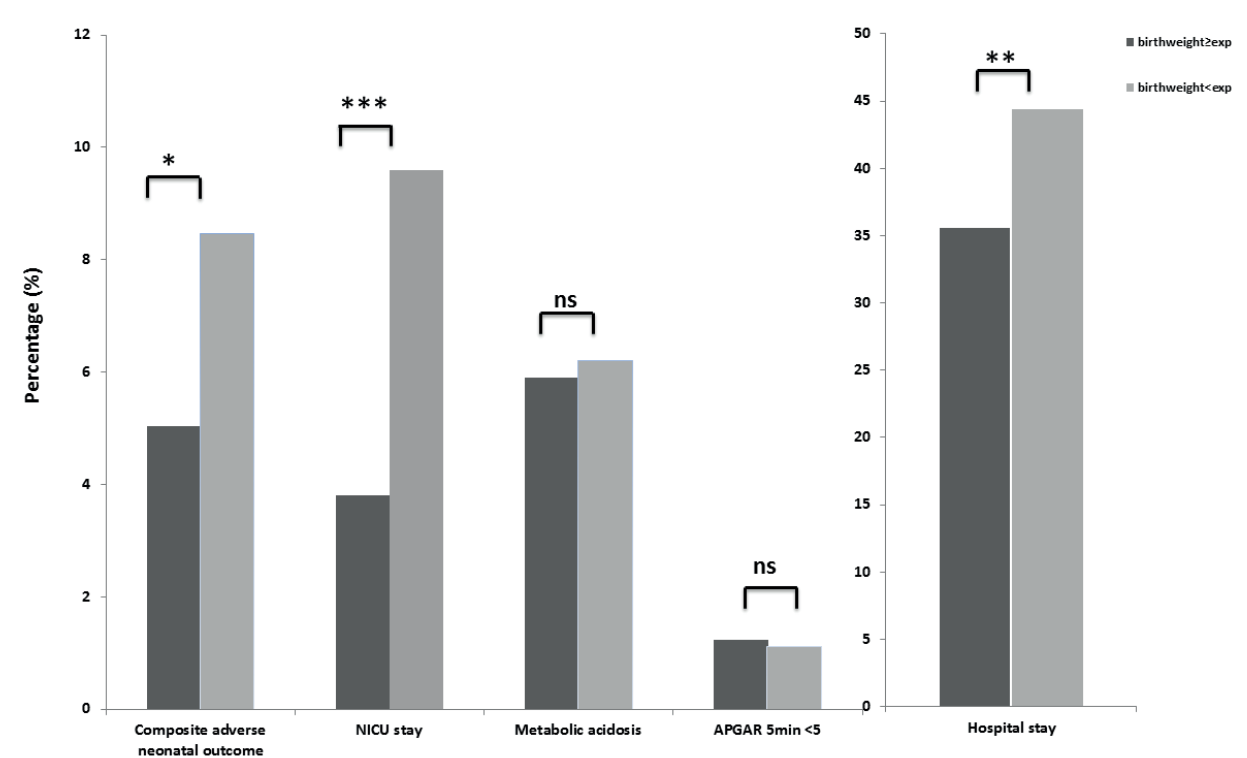

Figure 3 Bar chart of neonatal outcomes in birth weight $\geq$ expected and birth weight $<$ expected Data are expressed as $n$ (\%). Composite adverse neonatal outcome: asphyxia, sepsis, respiratory distress syndrome and transient tachypnoea of the newborn; NICU, neonatal intensive care unit. Metabolic acidosis, blood $\mathrm{pH}<7.0$ and base deficit $>12 \mathrm{mmol} / \mathrm{L}$

Birth weight $\geq$ expected (dark bars), birthweight $<$ expected (light bars). P-values are given, ns=not significant, ${ }^{*}=P<0.05,{ }^{* *}=P<0.01,{ }^{* * *}=P<0.001$

\section{DISCUSSION}

\section{Main findings}

In this retrospective study of non-anomalous singleton pregnancies, we investigated neonatal outcomes in a large cohort of neonates assumed to be 'appropriate-for-gestational-age' (AGA) (birth weight centiles between 10-80) in relation to their antenatal growth velocities. We aimed at identifying a subgroup within AGA neonates with reduced growth velocity, but who were not classified as small or growth restricted as their birth weight was above the 10th centile according to the Dutch perinatal registry system. In this study, these AGA neonates with suboptimal birth weight (arbitrarily defined as birth weight p10-50) had reduced velocities of all fetal growth parameters compared to the optimal AGA reference 
group (birth weight p50-80). Although we could not detect significant differences in neonatal outcomes based on birth weight categories, neonates with a composite adverse neonatal outcome and NICU stay had significantly lower growth velocities in the abdominal circumference, head circumference and biparietal diameter velocity compared with healthy neonates. These data suggest that abnormal fetal growth velocities are associated with adverse neonatal outcomes of presumed appropriate-for-gestational-age neonates due to suboptimal fetal growth.

\section{Strengths and limitations}

Strengths of this study were the large sample size and the comprehensive registration of ultrasound, obstetric and neonatal parameters in a single tertiary centre. Another strength of this study is the introduction of a new and practical method of assessing fetal growth potential within the AGA group using a simple calculation that can be readily employed and interpreted in low-resource setting without the need of complex mathematical models. An important limitation of the study is the retrospective design, with the inherent risk of selection bias and the exclusion of many cases in which the ultrasound examination did not fall within the specified time periods. Another limitation is the heterogeneity of the study population with various risk profiles for IUGR, as the study was performed in a tertiary referral centre. There was inadequate recording of determinants of obstetric risk and other confounders (such as ethnicity) to enable post-hoc stratification. This might limit the interpretation of our data and the generalizability to an unselected low-risk population. Caution must also be taken in interpreting our data as the association of fetal growth velocities with neonatal birthweight and adverse outcome does not necessarily imply the ability of such ultrasound parameters to identify individuals at risk for adverse outcomes. The degree of overlap in the min-max range of these velocities between normal and abnormal groups, especially in a large sample as in this study, weakens the predictive power of such parameters [23].

\section{Interpretation}

Undiagnosed fetal growth restriction is recognized as a major contributor to stillbirths and perinatal morbidity [24, 25]. Traditionally, the use of the 10th birth weight percentile to define SGA (as a proxy to neonates with growth restriction) has failed to reduce perinatal morbidity in the past decades, despite major improvement in neonatal and obstetric care [7]. This is undoubtedly related to failure to recognize AGA fetus who are 'at risk' of adverse outcome because of suboptimal growth by current standards [6]. We excluded SGA neonates (birth weight below the 10th centile) from the analysis, as this group already receives 
enough attention, although it is tempting to speculate that fetal growth velocities is reduced even further in severe FGR compared to constitutionally small neonates. In an effort to include only fetus with normal growth velocity, we set up the upper cut-off value for AGA neonates at the 80t centile. Although LGA neonates is traditionally defined as $\mathrm{BW}>90^{\text {th }}$ centile, neonates with BW between $80-90^{\text {th }}$ centile could potentially include fetus with pathologically accelerated growth due to e.g. gestational diabetes.

Determining optimum fetal growth is the cornerstone of proposing strategies for antenatal prediction and surveillance of pregnancies at risk of fetal growth restriction. Alas, this is an intricate and complex concept with competing and interacting influences from genetic, epigenetic and environmental backgrounds [26]. Several authors addressed this topic in reviews, editorials and observational trials, proposing different methods to identify fetus who fail to maintain their growth trajectory, such as individualized growth assessment (IGA) [11, 27], AC velocity [28], AC z-score [15] or conditional AC [14, 29]. In this study, although, all ultrasound parameters of fetal growth were reduced in suboptimal AGA neonates and those with an adverse neonatal outcome, we propose AC velocity as a practical and interpretable parameters to use in future studies. Abdominal circumference is the most appropriate ultrasonic measurement for the prediction of growth restriction in high-risk subjects [29,30], it is a specific marker for small-for-gestational-age [31] fetuses and, in the lowest decile, it can be used to predict adverse neonatal outcome [15]. We also discuss using the term "suboptimal appropriatefor gestational-age"(SAGA) to define those fetus who have possibly failed to reach their genetically predetermined growth potential. The available methods to chart fetal growth show considerable differences [32] and there is no consensus among authorities on the best chart to implement. The INTERGROWTH-21st study formulated ultrasound-based, universally fetal growth charts, corrected for different countries and ethnicities, and the World Health Organization published international fetal growth charts, based on multinational data [33]. However, both charts reflect population standards rather than individualized growth standards for a particular fetus/neonate, and a more accurate approach would consider each fetus as its own individual control by computing growth potential based on an early growth trajectory. Comparing actual birth weight or ultrasound parameters of fetal growth to this expected individualised growth standard was shown to correlate to neonatal outcome and placental pathology and can potentially improve the differentiation between true FGR and constitutional smallness [10, 11, 34]. Future studies comparing these different strategies should deliver the verdict on which standards to be followed internationally for an improved and modern definition of normal and abnormal fetal growth. 
This study highlights the importance of performing a third trimester biometry scan in an unselected population to determine target fetal growth. In the Netherlands, third trimester biometry scans are scheduled only for women who are at high risk of fetal growth restriction, according to national guidelines and agreements between gynaecologists and primary care midwives. There is ample evidence suggesting that the detection rate of small-for-gestational age improves with the additional scan in the third trimester [35] and triples the detection of neonatal morbidity in small-for-gestational age neonates [15]. An additional scan in appropriate-for-gestational-age neonates gives more insight in an eventually slowing fetal growth trajectory, which can be used as an indicator for fetal wellbeing [36]. It is unclear, however, when a third trimester biometry scan should be scheduled to improve the predictive power for adverse outcomes [28]. The addition of doppler measures of fetal hemodynamic compensation and biochemical measures of placental function to third trimester biometry scan is proposed to improve the performance of these tests for fetus at risk of intrapartum compromise and asphyxia $[37,38,39,40]$.

\section{CONCLUSION}

In conclusion, neonates with 'appropriate' birth weight for gestational age are a heterogeneous population, that includes a small 'at risk' group of fetuses who failed to reach their growth potential. How to tease out these apparently normal grown but 'relatively small for gestational age' fetus is a major challenge. Measuring abdominal circumference growth velocity in the third trimester, in combination with fetal dopplers and biomarkers of placenta function, could improve the identification of at risk fetus and are potential candidates for inclusion in multivariate models predicting fetal growth restriction.

\section{ABBREVIATIONS}

AGA: appropriate-for-gestational-age, SGA: small-for-gestational-age, AC: abdominal circumference, BPD: biparietal diameter, HC: head circumference, FL: femur length, FGR: fetal growth restriction, IUGR: intrauterine growth, EFW: expected fetal weight. NICU: neonatal intensive care unit 


\section{REFERENCES}

1. Smith-Bindman R, Chu PW, Ecker JL, Feldstein VA, Filly RA, Bacchetti P. US evaluation of fetal growth: prediction of neonatal outcomes. Radiology. 2002;223(1):153-61.

2. Figueras F, Gratacos E. An integrated approach to fetal growth restriction. Best Pract Res Clin Obstet Gynaecol. 2017;38:48-58.

3. Clausson B, Gardosi J, Francis A, Cnattingius S. Perinatal outcome in SGA births defined by customised versus population-based birthweight standards. BJOG. 2001;108(8):830-4.

4. Figueras F, Gratacos E. Update on the diagnosis and classification of fetal growth restriction and proposal of a stage-based management protocol. Fetal Diagn Ther. 2014;36(2):8698.

5. Conde-Agudelo A, Papageorghiou AT, Kennedy SH, Villar J. Novel biomarkers for predicting intrauterine growth restriction: a systematic review and meta-analysis. BJOG. 2013;120(6):681-94.

6. Francis JH, Permezel M, Davey MA. Perinatal mortality by birthweight centile. Aust N Z J Obstet Gynaecol. 2014;54(4):354-9.

7. Gardosi J, Madurasinghe V, Williams M, Malik A, Francis A. Maternal and fetal risk factors for stillbirth: population based study. BMJ. 2013;346:f108.

8. Bakalis S, Akolekar R, Gallo DM, Poon LC, Nicolaides KH. Umbilical and fetal middle cerebral artery Doppler at 30-34 weeks' gestation in the prediction of adverse perinatal outcome. Ultrasound Obstet Gynecol. 2015;45(4):409-20.

9. Papageorghiou AT, Ohuma EO, Altman DG, Todros T, Cheikh Ismail L, Lambert A, et al. International standards for fetal growth based on serial ultrasound measurements: the Fetal Growth Longitudinal Study of the INTERGROWTH-21st Project. Lancet. 2014;384(9946):869-79.

10. Deter RL, Lee W, Sangi-Haghpeykar H, Tarca AL, Li J, Yeo L, et al. Personalized third-trimester fetal growth evaluation: comparisons of individualized growth assessment, percentile line and conditional probability methods. J Matern Fetal Neonatal Med. 2016;29(2):177-85.

11. Deter RL, Lee W, Yeo L, Erez O, Ramamurthy U, Naik M, et al. Individualized growth assessment: conceptual framework and practical implementation for the evaluation of fetal growth and neonatal growth outcome. Am J Obstet Gynecol. 2018;218(2S):S656-S78.

12. Robinson HP. Sonar measurement of fetal crown-rump length as means of assessing maturity in first trimester of pregnancy. Br Med J. 1973;4(5883):28-31.

13. Salomon LJ, Alfirevic Z, Berghella V, Bilardo C, Hernandez-Andrade E, Johnsen SL, et al. Practice guidelines for performance of the routine mid-trimester fetal ultrasound scan. Ultrasound Obstet Gynecol. 2011;37(1):116-26.

14. Caradeux J, Eixarch E, Mazarico E, Basuki TR, Gratacos E, Figueras F. Second- to thirdtrimester longitudinal growth assessment for prediction of small-for-gestational age and late fetal growth restriction. Ultrasound Obstet Gynecol. 2018;51(2):219-24.

15. Sovio U, White IR, Dacey A, Pasupathy D, Smith GCS. Screening for fetal growth restriction with universal third trimester ultrasonography in nulliparous women in the Pregnancy Outcome Prediction (POP) study: a prospective cohort study. Lancet. 2015;386(10008):208997.

16. Hadlock FP, Harrist RB, Sharman RS, Deter RL, Park SK. Estimation of fetal weight with the use of head, body, and femur measurements-a prospective study. Am J Obstet Gynecol. 1985;151(3):333-7. 
17. Kloosterman GJ. [Intrauterine growth and intrauterine growth curves]. Maandschr Kindergeneeskd. 1969;37(7):209-25.

18. Cnattingius S, Norman M, Granath F, Petersson G, Stephansson O, Frisell T. Apgar Score Components at 5 Minutes: Risks and Prediction of Neonatal Mortality. Paediatr Perinat Epidemiol. 2017;31(4):328-37.

19. ACoO P. ACOG Committee opinion no. 348, November 2006: umbilical cord blood gas and acid-base analysis. . Obstet Gynecol. 2006;108(5):1319-22.

20. Sweet DG, Carnielli V, Greisen G, Hallman M, Ozek E, Plavka R, et al. European Consensus Guidelines on the Management of Respiratory Distress Syndrome - 2016 Update. Neonatology. 2017;111(2):107-25.

21. Buchiboyina A, Jasani B, Deshmukh M, Patole S. Strategies for managing transient tachypnoea of the newborn - a systematic review. J Matern Fetal Neonatal Med. 2017;30(13):152432.

22. Morales P, Bustamante D, Espina-Marchant P, Neira-Pena T, Gutierrez-Hernandez MA, Allende-Castro C, et al. Pathophysiology of perinatal asphyxia: can we predict and improve individual outcomes? EPMA J. 2011;2(2):211-30.

23. Pepe MS, Janes H, Longton G, Leisenring W, Newcomb P. Limitations of the odds ratio in gauging the performance of a diagnostic, prognostic, or screening marker. Am J Epidemiol. 2004;159(9):882-90.

24. Heazell AE, Worton SA, Higgins LE, Ingram E, Johnstone ED, Jones RL, et al. IFPA Gabor Than Award Lecture: Recognition of placental failure is key to saving babies' lives. Placenta. 2015;36 Suppl 1:S20-8.

25. Sebire NJ. Detection of fetal growth restriction at autopsy in non-anomalous stillborn infants. Ultrasound Obstet Gynecol. 2014;43(3):241-4.

26. Hanson M, Kiserud T, Visser GH, Brocklehurst P, Schneider EB. Optimal fetal growth: a misconception? Am J Obstet Gynecol. 2015;213(3):332 e1-4.

27. Deter RL, Lee W, Kingdom JCP, Romero R. Fetal growth pathology score: a novel ultrasound parameter for individualized assessment of third trimester growth abnormalities. J Matern Fetal Neonatal Med. 2018;31(7):866-76.

28. Salavati N, Sovio U, Mayo RP, Charnock-Jones DS, Smith GC. The relationship between human placental morphometry and ultrasonic measurements of utero-placental blood flow and fetal growth. Placenta. 2016;38:41-8.

29. Hutcheon JA, Egeland GM, Morin L, Meltzer SJ, Jacobsen G, Platt RW. The predictive ability of conditional fetal growth percentiles. Paediatr Perinat Epidemiol. 2010;24(2):131-9.

30. Chang TC, Robson SC, Boys RJ, Spencer JA. Prediction of the small for gestational age infant: which ultrasonic measurement is best? Obstet Gynecol. 1992;80(6):1030-8.

31. Seravalli V, Block-Abraham DM, Turan OM, Doyle LE, Blitzer MG, Baschat AA. Secondtrimester prediction of delivery of a small-for-gestational-age neonate: integrating sequential Doppler information, fetal biometry, and maternal characteristics. Prenat Diagn. 2014;34(11):1037-43.

32. Salomon LJ, Bernard JP, Duyme M, Buvat I, Ville Y. The impact of choice of reference charts and equations on the assessment of fetal biometry. Ultrasound Obstet Gynecol. 2005;25(6):559-65.

33. Kiserud T, Piaggio G, Carroli G, Widmer M, Carvalho J, Neerup Jensen L, et al. The World Health Organization Fetal Growth Charts: A Multinational Longitudinal Study of Ultrasound Biometric Measurements and Estimated Fetal Weight. PLoS Med. 2017;14(1):e1002220. 
34. Hata T, Kuno A, Akiyama M, Yanagihara T, Manabe A, Miyazaki K. Detection of smallfor-gestational-age infants with poor perinatal outcomes using individualized growth assessment. Gynecol Obstet Invest. 1999;47(3):162-5.

35. Lesmes C, Gallo DM, Saiid Y, Poon LC, Nicolaides KH. Prediction of small-for-gestationalage neonates: screening by uterine artery Doppler and mean arterial pressure at 19-24 weeks. Ultrasound Obstet Gynecol. 2015;46(3):332-40.

36. Bardien N, Whitehead CL, Tong S, Ugoni A, McDonald S, Walker SP. Placental Insufficiency in Fetuses That Slow in Growth but Are Born Appropriate for Gestational Age: A Prospective Longitudinal Study. PLoS One. 2016;11(1):e0142788.

37. Khalil A, Morales-Rosello J, Townsend R, Morlando M, Papageorghiou A, Bhide A, et al. Value of third-trimester cerebroplacental ratio and uterine artery Doppler indices as predictors of stillbirth and perinatal loss. Ultrasound Obstet Gynecol. 2016;47(1):74-80.

38. Morales-Rosello J, Khalil A. Fetal cerebral redistribution: a marker of compromise regardless of fetal size. Ultrasound Obstet Gynecol. 2015;46(4):385-8.

39. Morales-Rosello J, Khalil A, Morlando M, Bhide A, Papageorghiou A, Thilaganathan B. Poor neonatal acid-base status in term fetuses with low cerebroplacental ratio. Ultrasound Obstet Gynecol. 2015;45(2):156-61.

40. Morales-Rosello J, Khalil A, Morlando M, Papageorghiou A, Bhide A, Thilaganathan B. Changes in fetal Doppler indices as a marker of failure to reach growth potential at term. Ultrasound Obstet Gynecol. 2014;43(3):303-10. 



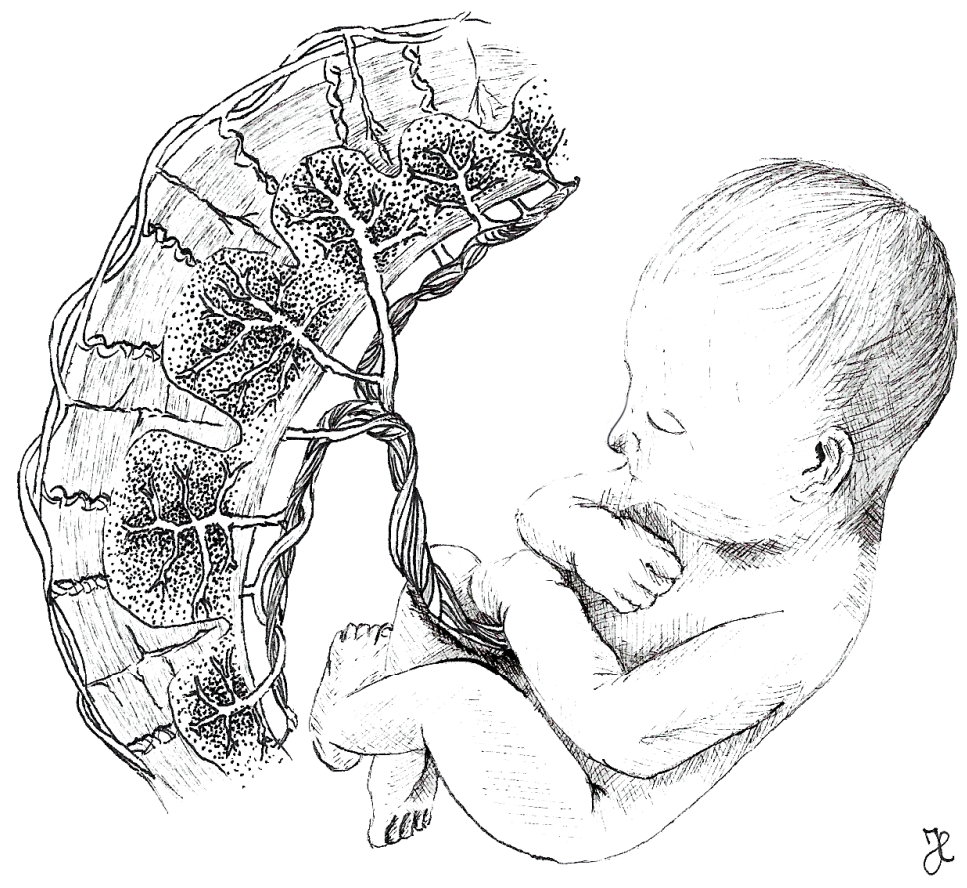




\title{
3 Can fetal growth velocity and first trimester maternal biomarkers improve the prediction of SGA and adverse neonatal outcome?
}

\author{
MLE Hendrix \\ JAP Bons \\ RRG Snellings \\ O Bekers \\ SMJ van Kuijk \\ MEA Spaanderman \\ S Al-Nasiry
}

Fetal Diagnosis and Therapy. 2019;46(4):274-284. 


\section{ABSTRACT}

\section{Background and objectives}

The aim of this study was to evaluate the value of adding fetal growth velocity and first trimester maternal biomarkers to baseline screening, for the prediction of small-for-gestational age (SGA) and adverse neonatal outcomes.

\section{Methods}

A retrospective cohort study was conducted of singleton pregnancies in the Maastricht University Medical Centre between 2012 and 2016. The biomarkers PAPP-A, $\beta$-hCG, PlGF, and sFlt-1 were measured at 11-13 weeks of gestational age (GA) and two fetal growth scans were performed (18-22 and 30-34 weeks of GA). Differences in biomarkers and growth velocities were compared between appropriate-for-gestational age (AGA; birth weight percentile 10-90) and SGA (birth weight percentile $<10$ ). Combinations of the biomarkers and fetal growth velocity were added to baseline screening for the prediction of SGA and adverse neonatal outcome.

\section{Results}

We included 296 singleton pregnancies. Compared to AGA $(\mathrm{n}=251)$, SGA neonates $(\mathrm{n}=45)$ had significantly lower growth velocities in the abdominal circumference (mm/week): $10.1 \pm 0.98$ versus $10.8 \pm 0.98, \mathrm{p}=0.001$. Compared with AGA, the SGA neonates had higher sFlt-1 multiples of the median (MoM): 0.89 (0.55) versus 0.76 (0.44), $\mathrm{p}=0.023$, and a higher sFlt-1/P1GF MoM ratio: 1.09 (1.03) versus 0.90 (0.64), $\mathrm{p}=0.027$. For a $15 \%$ false-positive rate, the prediction of SGA neonates increased from $44.8 \%$ for the baseline screening model to $56.5 \%$ after the addition of fetal growth velocities, and to $73.9 \%$ after the further addition of maternal biomarkers (PPV 9.6\%, NPV 82.4\%). The corresponding AUC for the three models were $0.722,0.804$, and 0.839 , respectively. In addition, AGA neonates with reduced fetal growth velocity had more adverse neonatal outcomes compared to the AGA reference group (12.4 vs. 3.9\%, $\mathrm{p}=0.013)$.

\section{Conclusion}

Combining fetal growth velocity with first trimester biomarkers resulted in a better prediction of SGA compared to baseline screening parameters alone. This approach could possibly result in reduced adverse neonatal outcomes in neonates, who are at a potential risk due to late mild placental dysfunction. 


\section{INTRODUCTION}

Undetected fetal growth restriction (FGR) is a major cause of perinatal morbidity. Fetuses with FGR have an increased risk of various complications, such as perinatal death, impaired neurodevelopment and several handicaps. In adult life they have an increased risk of manifestation of the metabolic syndrome, type 2 diabetes, coronary heart disease and hypertension [1-8]. Antenatal diagnosed FGR is associated with substantially reduced risks of adverse outcome compared to FGR diagnosed only after birth [9]. Despite advances in fetal ultrasound scanning techniques, early detection of FGR is still difficult. An expected birth weight below the $10^{\text {th }}$ percentile can be used to define small-for-gestational age (SGA) fetuses. However, the SGA group contains fetuses with FGR but also constitutionally small fetuses. What makes it even harder is that some fetuses in the appropriate-forgestational-age (AGA) group decline in growth velocity and have a form of FGR that falls outside of the contemporary definition and is thus unrecognized.

Currently, the criteria for diagnosing FGR are mainly based on the estimated fetal weight and Doppler hemodynamic $[10,11]$. Routine third trimester biometry measurements could improve the detection rate of FGR in the SGA and AGA group [12]. There is histological evidence that in small-for-gestational-age fetuses impaired placentation due to inadequate trophoblastic invasion of the maternal spiral arteries has taken place [13-15]. Inadequate trophoblastic invasion will lead to insufficient remodelling of the spiral arteries and this leads to a placental bed still susceptible for sympathetic stimuli and with it, decreased perfusion. Angiogenic factors such as placental growth factor (PIGF) are believed to regulate the above mentioned process [16]. PlGF and its inhibitor, the anti-angiogenic soluble fms-like tyrosine kinase-1 (sFlt-1), play an important role in the angiogenesis and placental development, with low concentrations of PIGF and high concentrations of sFlt-1 seen in SGA fetuses [17-21]. In addition, beta-human chorionic gonadotropin ( $\beta$-hCG) and pregnancy-associated plasma protein A (PAPP-A) are also being associated with preeclampsia, but have also been investigated regarding their association with SGA [22, 23].

PlGF belongs to the vascular endothelial growth factor family. It is expressed by trophoblast cells, placental villi and human umbilical vein endothelial cells. In early placental vascular development PlGF plays a role in potent pro-angiogenic effects. Reduced placental perfusion and subsequent placental bed hypoxia may be associated with low maternal plasma concentration of PIGF and delivery of a neonate with fetal growth restriction [24]. sFlt-1 is a splice variant of the vascular endothelial growth factor receptor Flt1 and a potent inhibitor of vascular endothelial growth factor (VEGF). Pregnancy-associated plasma protein A (PAPP-A) is a 
metalloprotease in the insulin-like growth factor (IGF) system. PAPP-A increases IGF bioavailability through regulated cleavage of IGF-binding protein 4 (IGFBP4) and is therefore a critical growth regulatory factor during fetal development [25]. Low PAPP-A levels are associated with chromosomal anomalies in fetuses, but it can also be used to predict adverse pregnancy outcomes such as fetal growth restriction [26].

Many studies describe the role of PlGF, sFlt- 1 and PAPP-A in the detection of pre-eclampsia, but their role in the prediction of FGR is less clear. Recent studies demonstrate that pregnancies resulting in the birth of SGA infants show similarly decreased levels of maternal PIGF and PAPP-A at $11+0$ to $13+6$ weeks of gestational age [22, 27-29]. There is also an association seen between FGR and lower PAPP-A and $\beta$-hCG values at 8 to 14 weeks of gestational age [26]. In addition, a high sFlt-1/ PIGF ratio in pregnant women is associated with adverse neonatal outcomes [30]. However, the difference between SGA and FGR in biomarker profile is still unclear. In this present study, we present differences between the categories of birth weight percentiles in the maternal biochemical biomarkers: PAPP-A, $\beta$-hCG, PlGF and sFlt-1, all assessed at the first trimester of pregnancy and ultrasound growth velocities parameters of the second and third trimester. We also combined the maternal biochemical biomarkers with ultrasound growth parameters. And the purpose of this study was to identify to what extent these parameters are able to detect intra uterine growth restriction and adverse neonatal outcome in smalland appropriate-for-gestational-age neonates.

\section{MATERIALS AND METHODS}

\section{Study design and participants}

In this retrospective cohort study pregnant women were included at the Maastricht University Medical Centre during the period of October 2012 to June 2016. Women were only included in this cohort if they had a singleton pregnancy without congenital anomalies, measurements of PAPP-A, $\beta$-hCG, PlGF and sFlt-1 at 11-13 weeks and if at least two fetal growth scans were available (one between 18 and 22 weeks of gestational age and one between 30 and 34 weeks of gestational age).

The pregnancies were dated according to the formula of Robinson calculated from the crown-rump length measurement in the first trimester [31]. We excluded women who had an infant with a birth weight above the $90^{\text {th }}$ percentile (large-for GA) because we wanted to predict adverse outcome. We defined a 'normal' reference group with appropriate for GA birth weights. Patients were informed about 
research at this university medical centre, and were given information about the data collection from their records for research purpose. Patients who refused to allow the use of their records were excluded from the study. The study protocol was approved by the medical ethical committee of the Maastricht University Medical Centre (17-4-0.15.1/ab). All procedures were in accordance with institutional guidelines and adhered to the principles of the Declaration of Helsinki and Title 45, U.S. Code of Federal Regulations, Part 46, Protection of Human Subjects (revised 13 November 2001, effective 13 December 2001). Consent to participate was waived because of the retrospective study design.

\section{Sampling of blood serum}

For all patients, blood samples were collected at week 11-13 of GA. All blood biomarkers (PlGF, sFlt-1, PAPP-A, and $\beta$-hCG) were measured in serum. Samples were analysed in a certified laboratory using commercially available kits. Measurements of sFlt-1 and PIGF assays were performed according to the manufacturer's instructions on the fully automated BRAHMS KRYPTOR compact PLUS system (ThermoFisher Scientific, Hennigsdorf, Germany). Both assays are immunometric assays based on the Time Resolved Amplified Cryptate Emission (TRACE) technology as described elsewhere [32]. Measurements of PAPP-A and free $\beta$-hCG were performed according to the manufacturer's instructions on the (fully automated) Auto-Delfia system (PerkinElmer,Turku, Finland). Both assays are fluoroimmunometric assays.

\section{Data analysis}

Maternal data were collected during routine hospital visits. Maternal age, height, weight, smoking status, and medical and obstetric history were recorded. Multiples of the median (MoM) values were calculated according to the GA at measurement.

Ultrasound measurements of abdominal circumference (AC), biparietal diameter (BPD), head circumference (HC) and femur length (FL) were recorded by an experienced sonographist, using a GE Voluson ultrasound machine, with a 2-to $5-\mathrm{MHz}$ curved-array transducer, in accordance with the routine mid-trimester fetal ultrasound scan guidelines [33]. These fetal growth scans were performed in two periods, one around 20 weeks (18-22 weeks) of GA during the second trimester anomaly scan, and one around 32 weeks (30-34 weeks) as a routine third trimester growth scan [34, 35]. Fetal growth parameters were recorded in an electronic database (Astraia GMBH). The estimated fetal weight was calculated using the Hadlock equation [36] [Hadlock C; $\log (10) \mathrm{BW}=1.335-0.0034(\mathrm{AC})(\mathrm{FL})$ $+0.0316(\mathrm{BPD})+0.0457(\mathrm{AC})+0.1623(\mathrm{FL})]$. For the purpose of this article we calcu- 
lated velocities of the fetal growth parameters (in $\mathrm{mm} /$ week) as the difference in absolute value of the measured parameters between the two examination periods, divided by the number of weeks.

Birth weight was quantified as percentiles according to the Dutch reference standard [37]. The percentiles were divided into two categories: (1) birth weight percentiles $<10$ as the SGA group, and (2) birth weight percentiles $10-90$ as the AGA group. The SGA and AGA fetuses were stratified into subgroups. The group with a reduced growth velocity of more than $20 \%$ in the AC velocity between 20 and 30 weeks were classified as fetuses with reduced velocity.

Potential predictors of SGA were tested in three models. The first model includes the baseline screening: maternal age, conception method (spontaneous vs. assisted), smoking during pregnancy (yes/no), previous SGA (yes/no), previous SGA stillbirth (yes/no), previous pre-eclampsia (yes/no), maternal BMI, first-trimester free $\beta$-hCG, first-trimester PAPP-A, AC at 32 (30-34) weeks of GA. The second model consists of the baseline screening (first model) and fetal growth velocities ( $\mathrm{mm} /$ week) of $\mathrm{AC}(\mathrm{ACv}), \mathrm{BPD}(\mathrm{BPDv}), \mathrm{HC}(\mathrm{HCv})$, and FL (FLv) between 20 and 32 weeks of GA. The third model consists of model 2, with the addition of MoM serum PlGF, sFlt- 1 and sFlt-1/PlGF ratio measured at 12 weeks of GA.

\section{Neonatal data}

Delivery and neonatal outcomes were registered by the obstetrician or in case of an adverse outcome by the paediatrician. APGAR-scores were assessed at 1 and $5 \mathrm{~min}$ after birth. We used a cut-off of less than 7 at $5 \mathrm{~min}$ to determine adverse outcome [38]. Metabolic acidosis was defined as an umbilical artery blood $\mathrm{pH}$ $<7.0$ and base deficit $>12 \mathrm{mmol} / \mathrm{L}$ ) [39]. Neonatal hypoglycaemia was defined as a plasma glucose level of less than $30 \mathrm{mg} / \mathrm{dL}(1.65 \mathrm{mmol} / \mathrm{L})$ in the first 24 hours of life and less than $45 \mathrm{mg} / \mathrm{dL}(2.5 \mathrm{mmol} / \mathrm{L})$ thereafter. The composite adverse neonatal outcome consists of 4 complications: asphyxia, sepsis, respiratory distress syndrome [40] and transient tachypnea of the newborn [41]. These complications were recorded in medical files and were defined according to the judgments of the attending medical staff. Asphyxia was defined as intrapartum-related hypoxiaischemia with multiple organ failure including encephalopathy [42]. Additionally, we recorded neonatal stay at the intensive care unit.

\section{Statistical analysis}

Baseline characteristics of the study sample were quantified as means with standard deviations (SD), median with range, and percentages with counts. Comparisons between the groups were made using the independent $t$ test or MannWhitney $U$ test for continuous data. For comparing categorical data between 
groups, the $\chi^{2}$ test was performed. Differences in circulating levels of biomarkers between the three groups were tested using one-way analysis of variance (ANOVA). Post-hoc tests were used to compare each two groups. Next, we computed odds ratios (OR), with upper and lower limits of $95 \%$ confidence intervals (CI), and the respective $p$ values were estimated separately for each potential predictor for SGA using logistic regression analysis. Second, each potential predictor was combined together in a multivariable logistic regression model. All the ORs were adjusted for GA, maternal age, BMI, parity and smoking status. The area under the receiver operating characteristic curve (AUC) was used to assess the discriminative ability of each factor. We tested the three models: baseline screening (model 1), with the addition of fetal growth velocities (model 2), and with the addition of both fetal growth velocities and biomarkers (model 3). The predicted probabilities from each regression model were saved as separate variables and their accuracy was assessed using receiver-operating characteristics (ROC) curve analysis. All analyses were performed using SPSS Statistics 23 (IBM Corp, Armonk, NY). $p$ values $\leq 0.05$ were considered to indicate statistical significance.

\section{RESULTS}

\section{Participants}

Between October 2012 and June 2016, we included 296 singleton pregnant women. The sample was divided into two categories: birth weight percentile $<10$ $(\mathrm{n}=45)$ as SGA and birth weight percentile $10-90(\mathrm{n}=251)$ as AGA. The general characteristics of the sample are given in Table 1. Women had a mean age of $32.7 \pm 4.6$ years and a mean body mass index of $23.9 \pm 4.6 \mathrm{~kg} / \mathrm{m} 2,49.3 \%$ were primiparous and $15.0 \%$ were smokers. Pre-eclampsia was present in $2.7 \%$ of the women in the total cohort. There were no statistically significant differences in GA at delivery, induction of labour, adverse neonatal outcome, or the proportion of neonates with an APGAR $<7$ at $5 \mathrm{~min}$.

The SGA group had fewer vaginal deliveries (71.1 vs. $87.3 \%, \mathrm{p}=0.006)$, more intrapartum emergency caesarean sections $(15.6 \%$ vs. $5.2 \%, \mathrm{p}=0.027)$, and more neonatal hypoglycaemia $(22.2 \%$ vs. $9.8 \%, \mathrm{p}=0.067)$, but there was no difference in rates of admission to the NICU (7.2 vs. $11.1 \%, \mathrm{p}=0.756)$.

\section{Differences in serum biomarkers and fetal growth velocities}

Table 2 shows the differences in first trimester maternal serum biomarkers and fetal growth velocities between SGA and AGA (reference group) neonates. SGA neonates, compared with AGA, had higher levels of sFlt-1 (pg/ml; 1,140 [703.1] 
Table 1. Baseline characteristics of the total cohort, reference group (birth weight percentiles 10-90) and study group (birth weight percentiles $<10$ ).

\begin{tabular}{|c|c|c|c|c|}
\hline & $\begin{array}{l}\text { Total study cohort } \\
\qquad(\mathrm{n}=296)\end{array}$ & $\begin{array}{c}\text { AGA (Bwp10-90) } \\
\text { (reference group) } \\
(\mathrm{n}=251)\end{array}$ & $\begin{array}{c}\text { SGA }(\mathrm{Bwp}<10) \\
(\text { study group }) \\
(\mathrm{n}=45)\end{array}$ & p-value \\
\hline Maternal age (years) & $32.7 \pm 4.6$ & $32.8 \pm 4.5$ & $32.0 \pm 4.7$ & 0.225 \\
\hline Primiparous (\%) & $49.3(146 / 296)$ & $46.6(117 / 251)$ & $64.4(29 / 45)$ & 0.017 \\
\hline BMI (kg/m2) & $23.9 \pm 4.6$ & $24.1 \pm 4.8$ & $23.1 \pm 3.7$ & 0.212 \\
\hline Smoking (yes/total) (\%) & $15.0(40 / 267)$ & $14.2(32 / 225)$ & $19.0(8 / 42)$ & 0.108 \\
\hline GA at birth (days): & $273(79)$ & $274(79)$ & $273(79)$ & 0.087 \\
\hline - GA <34wks (\%) & $1.7(5 / 296)$ & $1.2(3 / 251)$ & $4.4(2 / 45)$ & 0.167 \\
\hline - GA 34-36wks (\%) & $5.1(15 / 296)$ & $4.4(11 / 251)$ & $8.9(4 / 45)$ & 0.258 \\
\hline - GA >36wks (\%) & $93.2(276 / 296)$ & $9.4(237 / 251)$ & $86.7(39 / 45)$ & 0.097 \\
\hline AC $p<1020 w$ GA & $4.0(12 / 296)$ & $3.2(8 / 251)$ & $8.8(4 / 45)$ & 0.074 \\
\hline AC $\mathrm{p}<1030 \mathrm{w}$ GA & $9.1(27 / 296)$ & $4.8(12 / 251)$ & $33.3(15 / 45)$ & $<.0001$ \\
\hline Birth weight (g) & $3061.1 \pm 547.8$ & $3186.4 \pm 460.76$ & $2389.24 \pm 518.7$ & $<.0001$ \\
\hline Birth weight percentile & $38 \pm 26.8$ & $44 \pm 24.9$ & $6.0 \pm 2.9$ & $<.0001$ \\
\hline Previous SGA (\%) & $13.2(39 / 296)$ & $13.9(35 / 251)$ & $8.9(4 / 45)$ & 0.356 \\
\hline Previous SGA stillbirth (\%) & $0.7(2 / 296)$ & $0.8(2 / 251)$ & $0(0 / 45)$ & 0.548 \\
\hline Previous PE (\%) & $1.7(5 / 296)$ & $2.0(5 / 251)$ & $0(0 / 45)$ & 0.340 \\
\hline Preeclampsia (\%) & $2.7(8 / 296)$ & $1.6(4 / 251)$ & $8.9(4 / 45)$ & 0.021 \\
\hline Induction of labour (\%) & $32.4(96 / 296)$ & $31.1(78 / 251)$ & $40.0(18 / 45)$ & 0.299 \\
\hline \multicolumn{5}{|l|}{ Mode of delivery (\%) } \\
\hline - Vaginal & $84.8(251 / 296)$ & $87.3(219 / 251)$ & $71.1(32 / 45)$ & 0.006 \\
\hline - Prelabor caesarean & $8.4(25 / 296)$ & $7.6(19 / 251)$ & $13.3(6 / 45)$ & 0.122 \\
\hline - Intrapartum caesarean & $6.8(20 / 296)$ & $5.2(13 / 251)$ & $15.6(7 / 45)$ & 0.027 \\
\hline NICU stay (yes/total) (\%) & $5.7(16 / 282)$ & $11.3(12 / 106)$ & $11.1(4 / 36)$ & 0.093 \\
\hline APGAR <7 5min (\%) & $1.4(4 / 291)$ & $3.8(4 / 106)$ & $0(0 / 45)$ & 0.645 \\
\hline Metabolic acidosis (\%) & $6.2(14 / 225)$ & $6.7(13 / 194)$ & $3.2(1 / 31)$ & 0.699 \\
\hline Hypoglycaemia (\%) & $11.3(32 / 282)$ & $9.8(24 / 246)$ & $22.2(8 / 36)$ & 0.067 \\
\hline Adverse neonatal outcome (\%) & $7.8(22 / 282)$ & $7.2(18 / 251)$ & $11.1(4 / 36)$ & 0.756 \\
\hline
\end{tabular}

Data are given as mean $\pm S D$ (min-max), \% (n/N) or median (IQR) BMI, body mass index; GA, gestational age; AC, abdominal circumference; SGA, small-for-gestational age; PE, pre-eclampsia; NICU, neonatal intensive care unit. Hypoglycaemia, a plasma glucose level of less than $30 \mathrm{mg} / \mathrm{dL}(1.65 \mathrm{mmol} / \mathrm{L})$ in the first 24 hours of life and less than $45 \mathrm{mg} / \mathrm{dL}(2.5 \mathrm{mmol} / \mathrm{L})$ thereafter; Metabolic acidosis, umbilical artery blood $\mathrm{pH}<7.0$ and base deficit $>12 \mathrm{mmol} / \mathrm{L}$; Composite adverse neonatal outcome, consists of 4 complications: asphyxia, sepsis, respiratory distress syndrome and transient tachypnea of the new born; Asphyxia, intrapartum-related hypoxiaischemia with multiple organ failure including encephalopathy.

vs. 969 [565.50], p=0.031), higher sFlt-1 (pg/ml) MoM (0.89 [0.55] vs. 0.76 [0.44], $\mathrm{p}=0.023$ ) and higher sFlt-1/PlGF ratio (37.06 [35.15] vs. 30.66 [21.76], $\mathrm{p}=0.033)$, but no significant differences in PAPP-A, PAPP-A MoM, $\beta$-hCG, $\beta$-hCG MoM, PlGF and PIGF MoM levels. Fetal growth velocities were also significantly lower in SGA 
Table 2. Maternal serum biomarker levels and fetal growth velocities of the total cohort, reference group (birth weight percentiles 10-90) and study group (birth weight percentiles <10).

\begin{tabular}{|c|c|c|c|c|}
\hline & $\begin{array}{l}\text { Total study cohort } \\
\qquad(\mathrm{n}=296)\end{array}$ & $\begin{array}{l}\text { AGA (Bwp10-90) } \\
\text { (reference group) } \\
(\mathrm{n}=251)\end{array}$ & $\begin{array}{c}\text { SGA }(\mathrm{Bwp}<10) \\
(\text { study group) }(\mathrm{n}=45)\end{array}$ & p-value \\
\hline PAPP-A (mU/1) & $2481 \pm 1580.5$ & 2200 (1804) & 2001 (2010) & 0.209 \\
\hline PAPP-A MoM & $1.0 \pm 0.60$ & $0.93(4)$ & $0.93(2)$ & 0.245 \\
\hline$\beta$-hCG (mU/1) & $46.3 \pm 31.73$ & 36.8 (241.99) & $41.0(148.61)$ & 0.682 \\
\hline$\beta$-hCG MoM & $1.28 \pm 0.86$ & $1.04(7.11)$ & $1.16(3.85)$ & 0.686 \\
\hline PlGF (pg/ml) & $34.73 \pm 15.97$ & 31.5 (99.60) & $29.7(115.80)$ & 0.483 \\
\hline PlGF MoM & $0.87 \pm 0.40$ & $0.78(0.43)$ & $0.74(0.43)$ & 0.483 \\
\hline sFlt-1 (pg/ml) & $1123.00 \pm 544.09$ & $969(565.50)$ & 1140 (703.1) & 0.031 \\
\hline sFlt-1 MoM & $0.87 \pm 0.43$ & $0.76(0.44)$ & $0.89(0.55)$ & 0.023 \\
\hline sFlt-1/PlGF (pg/ml) & $38.39 \pm 26.03$ & $30.66(21.76)$ & $37.06(35.15)$ & 0.033 \\
\hline sFlt-1/PlGF MoM & $1.11 \pm 0.77$ & $0.90(0.64)$ & 1.09 (1.03) & 0.027 \\
\hline ACv (mm/week) & $10.78 \pm 0.98$ & $10.82 \pm 0.98$ & $10.08 \pm 0.98$ & 0.001 \\
\hline BPDv (mm/week) & $3.00 \pm 0.29$ & $3.02 \pm 0.28$ & $2.78 \pm 0.29$ & $<.0001$ \\
\hline HCv (mm/week) & $10.48 \pm 0.82$ & $10.53 \pm 0.80$ & $10.01 \pm 0.83$ & 0.001 \\
\hline FLv (mm/week) & $2.47 \pm 0.23$ & $2.48 \pm 0.22$ & $2.34 \pm 0.24$ & 0.002 \\
\hline
\end{tabular}

Data are given as mean \pm SD (min-max) or median (IQR). PAPP-A, Pregnancy-associated plasma protein A; $\beta-h C G$, beta human chorionic gonadotropin; PlGF, Placental growth factor; sFlt-1, soluble fms-like tyrosine kinase 1; MoM, multiples of the median, adjusted for gestational age; $A C v$, abdominal circumference velocity; $\mathrm{HC}$, head circumference velocity; BPDv, biparietal diameter velocity; FLv, femur length velocity.

compared to AGA neonates: $\mathrm{ACv}, 10.08 \pm 0.98$ versus $10.82 \pm 0.98, \mathrm{p}=0.001$; $\mathrm{BPDv}$, $2.78 \pm 0.29$ versus $3.02 \pm 0.28, \mathrm{p}<0.001 ; \mathrm{HCv}, 10.01 \pm 0.83$ versus $10.53 \pm 0.80, \mathrm{p}=0.001$, and FLv, $2.34 \pm 0.24$ vs. $2.48 \pm 0.22$, $\mathrm{p}=0.002$.

\section{Prediction of SGA}

The sFlt-1, sFlt-1/PlGF ratio and growth velocities, all showed significant associations with SGA (Table 3). However, these were not significant after adjustment for GA, maternal age, BMI, parity and smoking status. The baseline screening model did not show any significant association with SGA.

The discriminative ability of the three different models as quantified by AUC is shown in Figure 1 and Table 4. For the prediction of SGA, the baseline screening (maternal age, conception method, smoking during pregnancy, previous SGA, previous SGA stillbirth, previous PE, maternal BMI, $\beta$-hCG, PAPP-A and AC at 32 weeks of GA), had an AUC of 0.722 (95\% CI 0.615-0.829). Adding growth velocities (ACv, BPDv, HCv, FLv) to the baseline screening improved the prediction of SGA with an AUC of 0.804 (95\% CI 0.700-0.909). Adding growth velocities and biomarkers (PlGF, sFlt-1, sFlt-1/P1GF ratio) to the baseline screening, improved the prediction of SGA even more with an AUC of 0.839 (95\% CI 0.746-0.932). 
Table 3. Univariate odds-ratio's (OR) and multivariate adjusted odds-ratio's (aOR) for the prediction of SGA using various parameters.

\begin{tabular}{lcccc}
\hline & $\begin{array}{c}\text { Univariate (OR) } \\
\text { SGA }\end{array}$ & p-value & Multivariate (aOR) & SGA \\
\hline Baseline screening model & 0.628 & 0.767 & 0.628 & 0.767 \\
\hline Growth velocities (mm/week) & $0.034(0.001-1.015)$ & 0.051 & $0.687(0.409-1.153)$ & 0.155 \\
\hline PlGF (pg/ml) & $0.992(0.971-1.014)$ & 0.483 & $1.019(0.991-1.048)$ & 0.191 \\
\hline sFlt-1 (pg/ml) & $1.001(1.000-1.001)$ & 0.036 & $1.000(0.999-1.001)$ & 0.675 \\
\hline sFlt-1/PlGF $(\mathrm{pg} / \mathrm{ml})$ & $1.015(1.003-1.026)$ & 0.016 & $1.021(0.999-1.044)$ & 0.062 \\
\hline
\end{tabular}

Data are given as mean $\pm S D$. Baseline screening model: maternal age, conception method, smoking during pregnancy, previous $S G A$, previous SGA stillbirth, previous PE, maternal BMI, $\beta-h C G$, PAPP-A, abdominal circumference at 32 wks of GA. PAPP-A, pregnancy-associated plasma protein; $\beta-h C G$, beta human chorionic gonadotropin; PIGF, placental growth factor; sFlt-1, soluble fms-like tyrosine kinase 1. Odds ratios are adjusted for gestational age, maternal age, BMI, parity and smoking status.

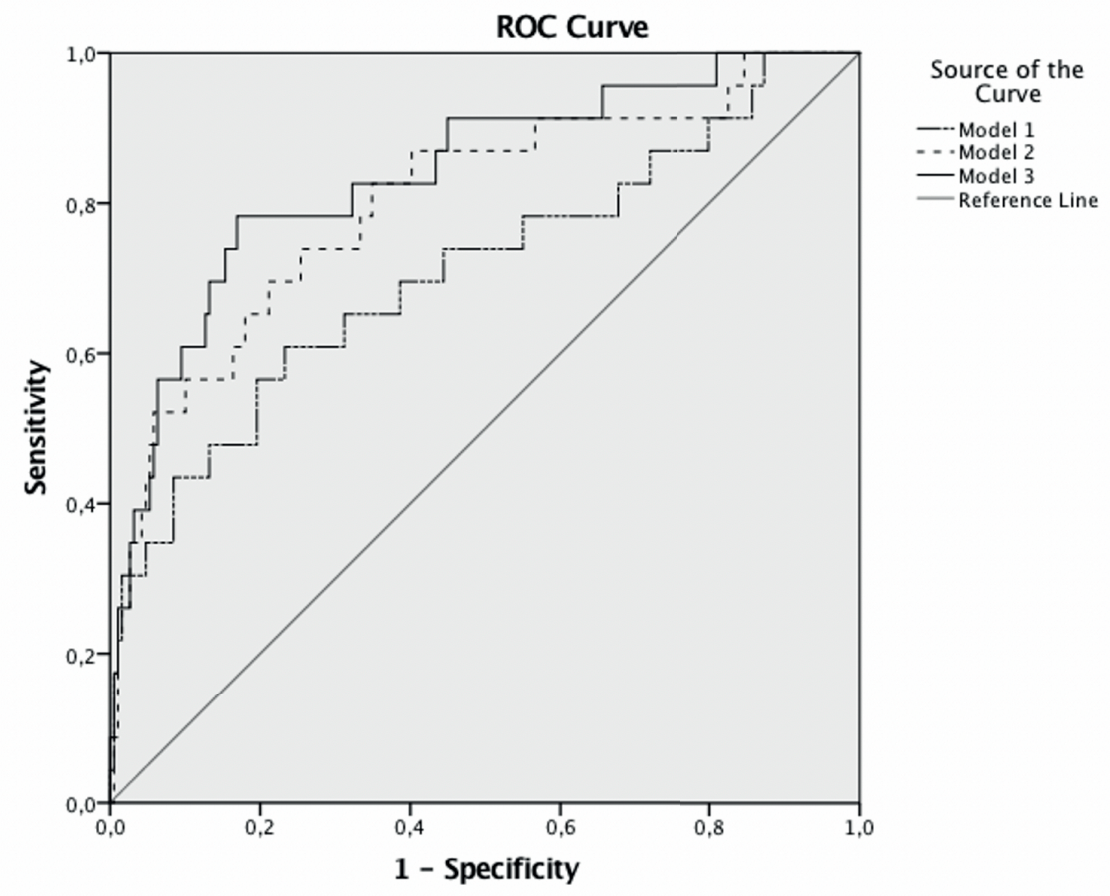

Figure 1 Receiver-operating characteristics (ROC) curve for prediction of SGA, using three different models. Model 1 baseline screening. Model 2 baseline screening plus fetal growth velocities. Model 3 baseline screening, fetal growth velocities and maternal biomarkers. 
Table 4. Predictive performance of three models for the prediction of SGA.

Model 1: baseline screening. Model 2: baseline screening with growth velocities. Model 3: baseline screening, growth velocities and maternal biomarkers.

\begin{tabular}{|c|c|c|c|}
\hline Predictive value & $\begin{array}{c}\text { Model } 1 \\
\text { Baseline screening }\end{array}$ & $\begin{array}{c}\text { Model } 2 \\
\text { BS + growth velocities }\end{array}$ & $\begin{array}{c}\text { Model } 3 \\
\text { BS + growth velocities }+ \\
\text { biomarkers }\end{array}$ \\
\hline AUC & $0.722(0.615-0.829)$ & $0.804(0.700-0.909)$ & $0.839(0.746-0.932)$ \\
\hline \multicolumn{4}{|l|}{$5 \%$ FPR } \\
\hline Sensitivity & $31.0(16.0-51.0)$ & $47.8(27.4-68.9)$ & $43.5(23.9-65.1)$ \\
\hline PPV & $4.6(2.3-8.8)$ & $5.7(3.0-10.1)$ & $5.3(2.7-9.7)$ \\
\hline NPV & 33.3 (17.9-52.9) & $45.4(25.1-67.3)$ & $40.9(21.5-63.3)$ \\
\hline \multicolumn{4}{|l|}{$10 \%$ FPR } \\
\hline Sensitivity & $41.4(24.1-60.9)$ & $56.5(34.9-76.1)$ & $60.9(38.8-79.5)$ \\
\hline PPV & $6.3(3.5-11.1)$ & $7.0(3.9-11.9)$ & $7.6(4.4-12.7)$ \\
\hline NPV & $54.1(37.1-70.2)$ & $65.5(45.7-81.4)$ & $67.9(47.6-83.4)$ \\
\hline \multicolumn{4}{|l|}{$15 \%$ FPR } \\
\hline Sensitivity & $44.8(27.0-64.0)$ & $56.5(34.9-76.1)$ & $73.9(51.3-88.9)$ \\
\hline PPV & $7.2(4.0-12.3)$ & $7.3(4.1-12.5)$ & $9.6(5.8-15.1)$ \\
\hline NPV & $65.2(49.7-78.2)$ & $74.4(57.6-86.4)$ & $82.4(64.8-92.6)$ \\
\hline \multicolumn{4}{|l|}{$20 \%$ FPR } \\
\hline Sensitivity & $55.2(36.0-73.0)$ & $69.6(47.0-85.9)$ & 78.3 (55.8-91.7) \\
\hline PPV & $9.2(5.5-14.8)$ & $9.4(5.6-15.1)$ & $10.7(6.6-16.5)$ \\
\hline NPV & $75.0(60.8-85.5)$ & 84.8 (70.5-93.2) & 88.4 (74.1-95.6) \\
\hline
\end{tabular}

Data are given as \% (95\% CI) and area under the curve (AUC) $(95 \% \mathrm{CI})$. PPV, positive predictive value; NPV, negative predictive value. Model 1: baseline screening: maternal age, conception method, smoking status, BMI, previous SGA, previous SGA stillbirth, previous preeclampsia; AC, abdominal circumference $<p 10$ at 32 weeks of gestational age, $\beta-h C G$ and PAPP-A at 12 weeks of gestational age. Model 2: baseline screening, abdominal circumference velocity, head circumference velocity, biparietal diameter velocity and femur length velocity between 20 and 32 weeks of gestational age. Model 3: model 2 with PlGF, sFlt-1 and sFlt-1/PlGF ratio at 12 weeks of gestational age.

For a $15 \%$ false-positive rate (FPR), the baseline screening model predicted $44.8 \%$ of SGA neonates. The addition of fetal growth velocities gave a prediction of 56.5\% for SGA. The combination of baseline screening, growth velocities, and maternal biomarkers gave a prediction of 73.9\% (PPV 9.6\%, NPV 82.4\%). The predictive performance of the three models for different FPRs is shown in Table 4.

\section{Stratification of outcome by reduced fetal growth}

The two groups (AGA and SGA) were both divided into two subgroups based on ACv between 20 and 30 weeks: AGA with normal growth consisting of 154 (52\%) neonates, as the reference group, AGA with reduced growth comprising 97 (33\%) neonates, SGA with normal growth consisting of $26(8.8 \%)$ neonates and SGA with reduced growth featuring 19 (6.4\%) neonates. 
SGA neonates with reduced growth showed lower PAPP-A $(\mathrm{p}=0.043)$ and $\beta$-hCG $(\mathrm{p}=0.027)$, higher sFlt- $1(\mathrm{p}=0.036)$ and ratio $(\mathrm{p}=0.029)$, compared with the AGA with normal growth (reference group). A similar trend was observed within the AGA neonates with reduced velocity. However, only PAPP-A was significantly different between the groups ( $\mathrm{p}=0.036$ ). SGA with normal growth had a higher sFlt-1/ PlGF ratio ( $\mathrm{p}=0.008$ ) compared to the reference group (Table 5). AGA with reduced velocity had significantly higher adverse neonatal outcomes (12.4\%) compared with AGA with normal growth $(3.9 \%, \mathrm{p}=0.013)$. Both categories of SGA neonates (with or without reduced AC velocity) had trends towards higher adverse neonatal outcome of 9.5 and 8.3\%, respectively (Table 6). The other neonatal outcomes were not significantly different. Fetal AC growth velocity had a modest predictive power for AGA with adverse neonatal outcome, with a sensitivity of $52.9 \%$ for a 20\% false-positive rate and AUC 0.716 (0.567-0.865) (Figure 2).

Table 5. Maternal serum biomarker levels in the AGA with normal growth (reference), AGA with reduced growth velocities, SGA with normal growth and SGA with reduced growth.

\begin{tabular}{|c|c|c|c|c|c|c|c|}
\hline & $\begin{array}{l}\text { AGA normal } \\
\text { growth } \\
\text { (reference) } \\
(\mathrm{n}=154)\end{array}$ & $\begin{array}{c}\text { AGA } \\
\text { reduced } \\
\text { growth } \\
(n=97)\end{array}$ & p-value & $\begin{array}{c}\text { SGA } \\
\text { normal } \\
\text { growth } \\
(\mathrm{n}=26)\end{array}$ & p-value & $\begin{array}{c}\text { SGA } \\
\text { reduced } \\
\text { growth } \\
(\mathrm{n}=19)\end{array}$ & p-value \\
\hline PAPP-A (mU/l) & $2284(2000)$ & $1997(1634)$ & 0.036 & 2199 (2431) & 0.405 & $1659(2130.3)$ & 0.043 \\
\hline$\beta$-hCG (mU/l) & $39.6(34.0)$ & $36.1(27.6)$ & 0.142 & $43.9(44.0)$ & 0.476 & 23.7 (29.73) & 0.027 \\
\hline PlGF (pg/ml) & 33.2 (18.95) & $30.8(15.25)$ & 0.067 & $30.4(19.63)$ & 0.487 & $29.7(13.20)$ & 0.238 \\
\hline sFlt-1 (pg/ml) & 980 (596.8) & 968 (550.9) & 0.240 & $1140(696.2)$ & 0.533 & $1171(913.5)$ & 0.036 \\
\hline sFlt-1/ PlGF ratio & $28.8(20.8)$ & $34.6(22.9)$ & 0.818 & $37.3(39.10)$ & 0.008 & 33.9 (31.89) & 0.029 \\
\hline
\end{tabular}

Data are given as median (IQR); PAPP-A, Pregnancy-associated plasma protein A; $\beta$-hCG, beta human chorionic gonadotropin; PIGF, placental growth factor; sFlt-1, soluble fms-like tyrosine kinase 1. Reduced growth is defined as a reduced growth velocity of more than 20 percent in the abdominal circumference velocity between 20 and 30 weeks. AGA, fetuses with a birth weight between the 10-90th percentile. SGA, fetuses with a birth weight below the $10^{\text {th }}$ percentile. 


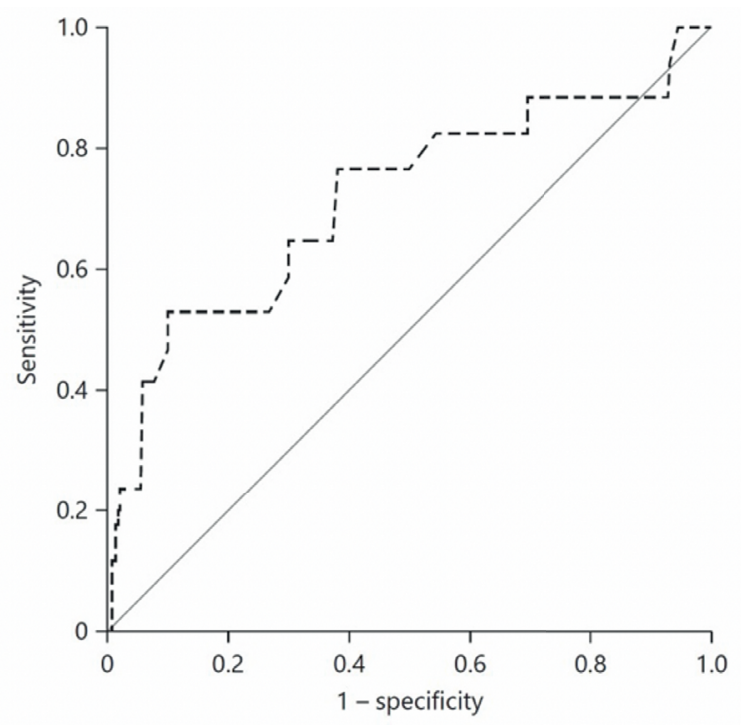

Figure 2 Receiver-operating characteristics (ROC) curve for prediction of AGA, with adverse neonatal outcome, using abdominal circumference growth velocity between 20 and 32 weeks of gestational age. Area under ROC curve was $0.716(0.567-0.865)$. Sensitivity of $52.9 \%$, with a FPR of $20 \%$.

Table 6. Neonatal outcome in the AGA with normal growth (reference), AGA with reduced growth velocities, SGA with normal growth and SGA with reduced growth.

\begin{tabular}{|c|c|c|c|c|c|c|c|}
\hline & $\begin{array}{l}\text { AGA normal } \\
\text { growth } \\
\text { (reference) } \\
(\mathbf{n}=154)\end{array}$ & $\begin{array}{c}\text { AGA } \\
\text { reduced } \\
\text { growth } \\
(n=97)\end{array}$ & P-value & $\begin{array}{c}\text { SGA } \\
\text { normal } \\
\text { growth } \\
(\mathrm{n}=26)\end{array}$ & P-value & $\begin{array}{c}\text { SGA } \\
\text { reduced } \\
\text { growth } \\
(\mathrm{n}=19)\end{array}$ & P-value \\
\hline NICU admission (yes/total) & $3.9 \%(6 / 154)$ & $6.2 \%(6 / 97)$ & 0.449 & $12.5 \%(3 / 26)$ & 0.093 & $9.5 \%(2 / 19)$ & 0.300 \\
\hline APGAR $<75 \mathrm{~min}$ & $1.3 \%(2 / 154)$ & $2.1 \%(2 / 97)$ & 0.617 & $0 \%(0 / 26)$ & 0.596 & $0 \%(0 / 19)$ & 0.644 \\
\hline Metabolic acidosis & $9.2 \%(11 / 119)$ & $2.7 \%(2 / 75)$ & 0.066 & $6.2 \%(1 / 16)$ & 0.642 & $0 \%(0 / 15)$ & 0.163 \\
\hline Hypoglycemia & $7.8 \%(12 / 154)$ & $12.4 \%(12 / 97)$ & 0.261 & $19.2 \%(5 / 26)$ & 0.087 & $21.1 \%(4 / 19)$ & 0.083 \\
\hline Adverse neonatal outcome & $3.9 \%(6 / 154)$ & $12.4 \%(12 / 97)$ & 0.013 & $8.3 \%(2 / 26)$ & 0.440 & $9.5 \%(2 / 19)$ & 0.355 \\
\hline
\end{tabular}

Data are given as \% (n/N). NICU, neonatal intensive care unit. Adverse neonatal outcome, asphyxia, sepsis, respiratory distress syndrome and transient tachypnea of the newborn. Hypoglycemia was defined as a plasma glucose level of less than $30 \mathrm{mg} / \mathrm{dL}$ (1.65 mmol/L) in the first 24 hours of life and less than $45 \mathrm{mg} / \mathrm{dL}(2.5 \mathrm{mmol} / \mathrm{L})$ thereafter. Asphyxia was defined as intrapartum-related hypoxia-ischemia with multiple organ failure including encephalopathy. 


\section{DISCUSSION}

\section{Main findings}

In this large retrospective study, we tested the additional value of first trimester maternal placental biomarkers and ultrasound fetal growth velocities to predict the birth of SGA neonates. This study showed that SGA neonates had reduced fetal growth velocities between the second and third trimester compared to AGA neonates, with an altered first trimester placental biomarker profile. The combination of the maternal biochemical biomarkers at 11-13 weeks and ultrasound growth velocities at 20 to 32 weeks improves the prediction of SGA neonates compared to baseline screening alone. By taking fetal growth velocities in consideration, the difference in biomarker profile was mainly seen in the subgroup of SGA with reduced velocity. Even the AGA group with reduced fetal growth had a lower PAPP-A and more adverse neonatal outcomes, compared with the AGA reference group.

\section{Strengths and limitations}

The strength of this study lies in the fact that the study population consists of all women during a certain period of time with a comprehensive registration of ultrasound, obstetric and neonatal parameters in a single tertiary centre. All women gave birth in the MUMC and had available maternal biochemical biomarkers and ultrasound growth velocities, all at the same GA. Furthermore, the levels of the biomarkers were measured at the end of the first trimester; this is a crucial moment for therapies, that can be initiated to possibly alter the course of placental dysfunction. Third, the measurement of the ultrasound growth velocities is a new and practical method of assessing fetal growth potential within the SGA and AGA group using a simple calculation that can be readily employed and interpreted in low-resource setting without the need of complex mathematical models. Another strength of this study is the focus mainly on SGA without PE, whereas other studies gave results of SGA combined with PE. In this study only $2 \%$ of the population had PE.

We acknowledge that there are some limitations to this study. First of all, the population consists of 296 neonates, which is rather small. The result would be of greater importance in a larger population. Second, the study had a retrospective design with the inherent risk of selection bias and the exclusion of many cases in which the ultrasound examination and the biomarkers did not fall within the specified time periods. Another limitation is the heterogeneity of study population with various risk profiles for FGR and the association with preeclampsia, as the study was performed in a tertiary referral centre. There was inadequate 
recording of determinants of obstetric risk and other confounders (such as ethnicity, maternal weight and length) to enable post-hoc stratification. This might limit the interpretation of our data and the generalizability to an unselected low-risk population.

\section{Interpretation}

The introduction of placental biomarkers (PAPP-A and $\beta$-hCG) into national aneuploidy screening programmes worldwide has soon led to the first citations of linking the maternal serum levels of these biomarkers in the first trimester to a variety of obstetric complications later in pregnancy [26]. This was not surprising taking into account the fact that these biomarkers are secreted by placental cells (villous syncytiotrophoblast) into the maternal circulation and that they play a crucial role in placental development and function [43]. Since preeclampsia is considered an epitome of placenta dysfunction, there has been ample literature on the use of placental biomarkers to predict the occurrence of preeclampsia, as well as its severity and its various phenotypes.

FGR has as strong link to placental dysfunction, as does preeclampsia. However, the arbitrary definitions used to define both intrauterine and postnatal growth abnormalities have led to confusing conclusions regarding the link to placental abnormalities, including placental biomarkers. The data on the association between FGR and $\beta$-hCG values in the first trimester are conflicting with both lower and higher $\beta$-hCG values being reported in FGR pregnancies, possibly depending on the GA at sampling. The conclusion is that serum $\beta$-hCG is not a reliable marker to predict either FGR or preeclampsia; however PAPP-A has good predictive power for both FGR and preeclampsia [8, 26].

Many studies demonstrated that pregnancies resulting in the birth of SGA infants, showed decreased PAPP-A at 11+0 to 13+6 weeks of GA [22, 27, 29, 44, 45]. Our data are consistent with these findings, showing that reduced PAPP-A and $\beta$-hCG levels were only observed in SGA pregnancies with reduced fetal growth.

The emergence of angiogenic factors as novel biomarkers of placental dysfunction has led to a surge in publications addressing their role as possibly the best biochemical predictors of $\mathrm{PE}$, coinciding with the implementation of their use in the clinical setting of triaging women with suspected preeclampsia. P1GF and sFlt-1 have apposing functions on endothelial cells. Lower P1GF levels and higher sFlt-1 levels have been linked to the development of PE and FGR [16, 21, $46,47]$. A ratio of sFlt-1/P1GF has been proposed to have a better predictive and discriminatory power compared to either biomarker alone, for various adverse neonatal outcomes [30, 48, 49]. Our data confirm that sFlt- 1 and the sFlt-1/PlGF ratio were higher in SGA pregnancies. Consequently, the predictive power of first 
trimester placental biomarkers, alone or in combination was higher for SGA than AGA. In the subgroup of SGA neonates with normal fetal growth, sFlt-1/PIGF ratio was higher compared to controls, suggesting general 'smallness', not necessarily abnormal placentation.

It is interesting to view the differences in biomarker data between SGA and AGA with a reduced fetal growth in our population from the perspective of divergent placental pathoaetiology. Evidence suggests that there are two different phenotypes of FGR. First, an early FGR phenotype resulting in severe SGA is associated with placental bed pathology and abnormal placental development, reflected by early changes in uteroplacental Doppler profile and placental serum biomarkers. The second is a late mild FGR phenotype, characterised by mild placental disease, a normal uteroplacental Doppler profile and abnormal hemodynamic fetal adaptation reflected by late cerebroplacental Doppler changes [10, 50]. Our data suggest that we could identify a subgroup with reduced fetal growth velocity within AGA neonates, who had both reduced PAPP-A levels and more adverse neonatal outcome. We therefore postulate that AGA neonates represent fetus with normal early placentation. However, they show reduced growth velocities between the second and third trimester and subsequently fail to achieve their optimal birth weight due to external factors limiting either placental capacity of nutritional supply. This study highlights the importance of performing a third trimester biometry scan in an unselected population to determine target fetal growth. This mild and late form of restricted fetal growth is often missed in the current clinical practise and poses a challenge to obstetricians to improve its detection and reduce the associated late fetal compromise [11].

\section{CONCLUSION}

The detection of FGR is still a major and complicated task, as the majority with pathological restriction of growth capacity due to placental dysfunction is not properly detected. Currently, the diagnosis of FGR is mainly based on a single measurement mainly performed in the second or third trimester. To maximize the detection of FGR, we recommend combining maternal serum placental biomarkers in the first trimester such as the ones given in this study; PlGF, sFlt1, PAPP-A and $\beta$-hCG with fetal growth velocities. This approach could possibly result in reduced morbidity in AGA neonates, who are at a potential risk due to late mild placental dysfunction. 


\section{REFERENCES}

1. S, M.K. and J. Gardosi, Perinatal mortality and fetal growth restriction. Best Pract Res Clin Obstet Gynaecol, 2004. 18(3): p. 397-410.

2. Pallotto, E.K. and H.W. Kilbride, Perinatal outcome and later implications of intrauterine growth restriction. Clin Obstet Gynecol, 2006. 49(2): p. 257-69.

3. Lees, C., et al., Perinatal morbidity and mortality in early-onset fetal growth restriction: cohort outcomes of the trial of randomized umbilical and fetal flow in Europe (TRUFFLE). Ultrasound Obstet Gynecol, 2013. 42(4): p. 400-8.

4. Leitner, Y., et al., Neurodevelopmental outcome of children with intrauterine growth retardation: a longitudinal, 10-year prospective study. J Child Neurol, 2007. 22(5): p. 580-7.

5. Barker, D.J., Adult consequences of fetal growth restriction. Clin Obstet Gynecol, 2006. 49(2): p. 270-83.

6. Crispi, F., et al., Fetal growth restriction results in remodeled and less efficient hearts in children. Circulation, 2010. 121(22): p. 2427-36.

7. Varvarigou, A.A., Intrauterine growth restriction as a potential risk factor for disease onset in adulthood. J Pediatr Endocrinol Metab, 2010. 23(3): p. 215-24.

8. Karagiannis, G., et al., Prediction of small-for-gestation neonates from biophysical and biochemical markers at 11-13 weeks. Fetal Diagn Ther, 2011. 29(2): p. 148-54.

9. Lindqvist, P.G. and J. Molin, Does antenatal identification of small-for-gestational age fetuses significantly improve their outcome? Ultrasound Obstet Gynecol, 2005. 25(3): p. 258-64.

10. Figueras, F. and E. Gratacos, Update on the diagnosis and classification of fetal growth restriction and proposal of a stage-based management protocol. Fetal Diagn Ther, 2014. 36(2): p. 86-98.

11. Figueras, F. and J. Gardosi, Intrauterine growth restriction: new concepts in antenatal surveillance, diagnosis, and management. Am J Obstet Gynecol, 2011. 204(4): p. 288-300.

12. Papageorghiou, A.T., et al., International standards for fetal growth based on serial ultrasound measurements: the Fetal Growth Longitudinal Study of the INTERGROWTH-21st Project. Lancet, 2014. 384(9946): p. 869-79.

13. Sheppard, B.L. and J. Bonnar, An ultrastructural study of utero-placental spiral arteries in hypertensive and normotensive pregnancy and fetal growth retardation. Br J Obstet Gynaecol, 1981. 88(7): p. 695-705.

14. Khong, T.Y., et al., Inadequate maternal vascular response to placentation in pregnancies complicated by pre-eclampsia and by small-for-gestational age infants. Br J Obstet Gynaecol, 1986. 93(10): p. 1049-59.

15. Pijnenborg, R., L. Vercruysse, and M. Hanssens, The uterine spiral arteries in human pregnancy: facts and controversies. Placenta, 2006. 27(9-10): p. 939-58.

16. Cowans, N.J., et al., First-trimester placental growth factor as a marker for hypertensive disorders and SGA. Prenat Diagn, 2010. 30(6): p. 565-70.

17. Rana, S., et al., Sequential changes in antiangiogenic factors in early pregnancy and risk of developing preeclampsia. Hypertension, 2007. 50(1): p. 137-42.

18. Erez, O., et al., The change in concentrations of angiogenic and anti-angiogenic factors in maternal plasma between the first and second trimesters in risk assessment for the subsequent development of preeclampsia and small-for-gestational age. J Matern Fetal Neonatal Med, 2008. 21(5): p. 279-87. 
19. Taylor, R.N., et al., Longitudinal serum concentrations of placental growth factor: evidence for abnormal placental angiogenesis in pathologic pregnancies. Am J Obstet Gynecol, 2003. 188(1): p. 177-82.

20. Crispi, F., et al., Placental angiogenic growth factors and uterine artery Doppler findings for characterization of different subsets in preeclampsia and in isolated intrauterine growth restriction. Am J Obstet Gynecol, 2006. 195(1): p. 201-7.

21. Romero, R., et al., A longitudinal study of angiogenic (placental growth factor) and antiangiogenic (soluble endoglin and soluble vascular endothelial growth factor receptor-1) factors in normal pregnancy and patients destined to develop preeclampsia and deliver a small for gestational age neonate. J Matern Fetal Neonatal Med, 2008. 21(1): p. 9-23.

22. Sung, K.U., et al., Maternal serum placental growth factor and pregnancy-associated plasma protein A measured in the first trimester as parameters of subsequent pre-eclampsia and small-for-gestational-age infants: A prospective observational study. Obstet Gynecol Sci, 2017. 60(2): p. 154-162.

23. Cignini, P., et al., Predictive value of pregnancy-associated plasma protein-A (PAPP-A) and free beta-hCG on fetal growth restriction: results of a prospective study. Arch Gynecol Obstet, 2016. 293(6): p. 1227-33.

24. Torry, D.S., et al., Placenta growth factor: potential role in pregnancy. Am J Reprod Immunol, 1999. 41(1): p. 79-85.

25. Conover, C.A., et al., Metalloproteinase pregnancy-associated plasma protein A is a critical growth regulatory factor during fetal development. Development, 2004. 131(5): p. 118794.

26. Smith, G.C., et al., Early pregnancy levels of pregnancy-associated plasma protein a and the risk of intrauterine growth restriction, premature birth, preeclampsia, and stillbirth. J Clin Endocrinol Metab, 2002. 87(4): p. 1762-7.

27. Thadhani, R., et al., First trimester placental growth factor and soluble fms-like tyrosine kinase 1 and risk for preeclampsia. J Clin Endocrinol Metab, 2004. 89(2): p. 770-5.

28. Boucoiran, I., et al., Risks for preeclampsia and small for gestational age: predictive values of placental growth factor, soluble fms-like tyrosine kinase-1, and inhibin A in singleton and multiple-gestation pregnancies. Am J Perinatol, 2013. 30(7): p. 607-12.

29. Tsiakkas, A., et al., Serum placental growth factor in the three trimesters of pregnancy: effects of maternal characteristics and medical history. Ultrasound Obstet Gynecol, 2015. 45(5): p. 591-8.

30. Chang, Y.S., et al., The sFlt-1/PlGF ratio as a predictor for poor pregnancy and neonatal outcomes. Pediatr Neonatol, 2017. 58(6): p. 529-533.

31. Robinson, H.P., Sonar measurement of fetal crown-rump length as means of assessing maturity in first trimester of pregnancy. Br Med J, 1973. 4(5883): p. 28-31.

32. Mathis, G., Rare earth cryptates and homogeneous fluoroimmunoassays with human sera. Clin Chem, 1993. 39(9): p. 1953-9.

33. Practice guidelines for performance of the routine mid-trimester fetal ultrasound scan. 2010.

34. Sovio, U., et al., Screening for fetal growth restriction with universal third trimester ultrasonography in nulliparous women in the Pregnancy Outcome Prediction (POP) study: a prospective cohort study. Lancet, 2015. 386(10008): p. 2089-97.

35. Caradeux, J., et al., Second to third trimester longitudinal growth assessment for the prediction of SGA and late FGR. Ultrasound Obstet Gynecol, 2017. 
36. Hadlock, F.P., et al., Estimation of fetal weight with the use of head, body, and femur measurements--a prospective study. Am J Obstet Gynecol, 1985. 151(3): p. 333-7.

37. Kloosterman, G.J., [Intrauterine growth and intrauterine growth curves]. Ned Tijdschr Verloskd Gynaecol, 1969. 69(5): p. 349-65.

38. Cnattingius, S., et al., Apgar Score Components at 5 Minutes: Risks and Prediction of Neonatal Mortality. Paediatr Perinat Epidemiol, 2017.

39. Practice, A.C.o.O., ACOG Committee Opinion No. 348, November 2006: Umbilical cord blood gas and acid-base analysis. Obstet Gynecol, 2006. 108(5): p. 1319-22.

40. Sweet, D.G., et al., European Consensus Guidelines on the Management of Respiratory Distress Syndrome - 2016 Update. Neonatology, 2017. 111(2): p. 107-125.

41. Buchiboyina, A., et al., Strategies for managing transient tachypnoea of the newborn - a systematic review. J Matern Fetal Neonatal Med, 2017. 30(13): p. 1524-1532.

42. Morales, P., et al., Pathophysiology of perinatal asphyxia: can we predict and improve individual outcomes? EPMA J, 2011. 2(2): p. 211-30.

43. D'Elia, P., et al., Homodimeric pregnancy-associated plasma protein-A in normal human placenta of first and third trimester of pregnancy: biochemical and morphological observations. Placenta, 2012. 33(11): p. 942-5.

44. Birdir, C., et al., Impact of maternal serum levels of Visfatin, AFP, PAPP-A, sFlt-1 and PIGF at 11-13 weeks gestation on small for gestational age births. J Matern Fetal Neonatal Med, 2017. 30(6): p. 629-634.

45. Vandenberghe, G., et al., First trimester screening for intra-uterine growth restriction and early-onset pre-eclampsia. Prenat Diagn, 2011. 31(10): p. 955-61.

46. Poon, L.C., et al., Maternal serum placental growth factor (PIGF) in small for gestational age pregnancy at 11(+0) to 13(+6) weeks of gestation. Prenat Diagn, 2008. 28(12): p. 1110-5.

47. Litwinska, E., et al., Combined screening for early and late pre-eclampsia and intrauterine growth restriction by maternal history, uterine artery Doppler, mean arterial pressure and biochemical markers. Adv Clin Exp Med, 2017. 26(3): p. 439-448.

48. Triunfo, S., et al., Changes in uterine artery Doppler velocimetry and circulating angiogenic factors in the first half of pregnancies delivering a small-for-gestational-age neonate. Ultrasound Obstet Gynecol, 2017. 49(3): p. 357-363.

49. Crovetto, F., et al., First-trimester screening with specific algorithms for early- and lateonset fetal growth restriction. Ultrasound Obstet Gynecol, 2016. 48(3): p. 340-8.

50. Savchev, S., et al., Evaluation of an optimal gestational age cut-off for the definition of early- and late-onset fetal growth restriction. Fetal Diagn Ther, 2014. 36(2): p. 99-105. 


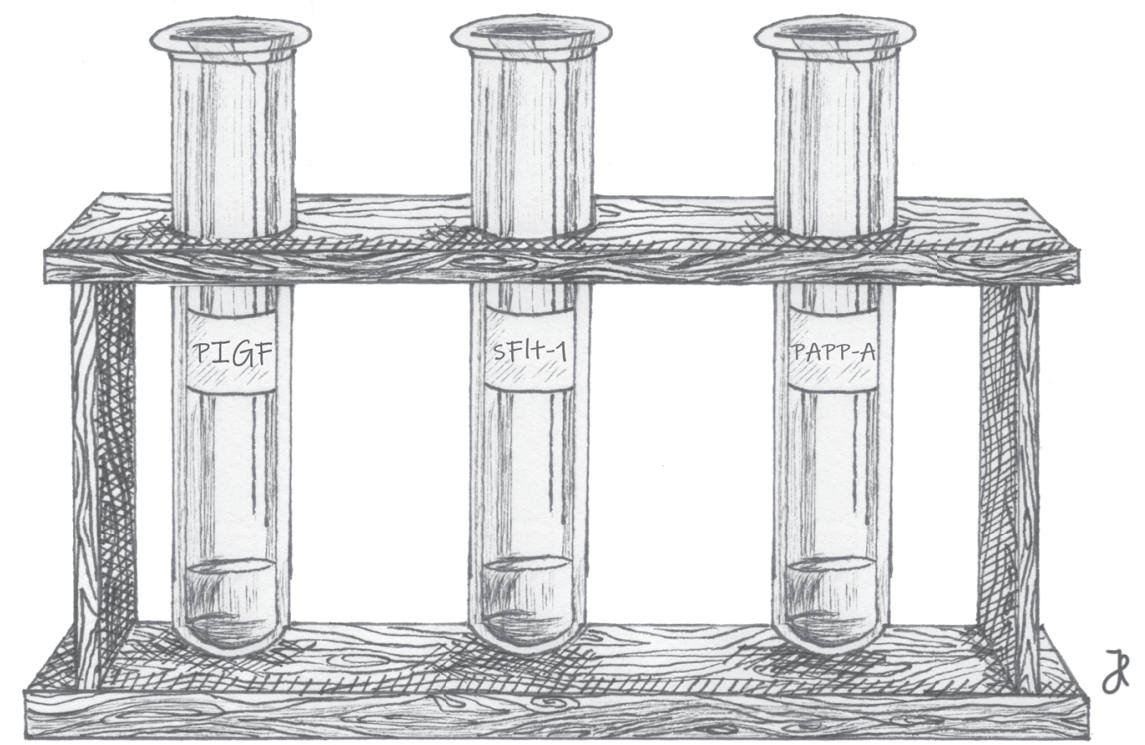




\section{Longitudinal changes in placental biomarkers in women with early versus late placental dysfunction.}

MLE Hendrix

KCM Palm

SMJ van Kuijk

O Bekers

MEA Spaanderman

JAP Bons

S Al-Nasiry

Hypertension in Pregnancy. 2019 Nov;38(4):268-277. 


\section{ABSTRACT}

\section{Objective}

To evaluate longitudinal changes of angiogenic biomarkers in early- (EO-PD) versus late-onset (LO-PD) placental dysfunction.

\section{Methods}

Serum PlGF and sFlt-1 measured at different intervals in EO-PD ( $\mathrm{n}=43$ ), LO-PD $(n=31)$ and controls $(n=133)$.

\section{Results}

sFtl-1/PlGF ratio was higher at 16 weeks (30.6 vs 17.5), 20 weeks (29.3 vs 8.9) and 30- weeks (16.6 vs 6.7) in EO-PD vs controls (all p<0.05), but not in LO-PD. Longitudinal changes for all intervals had higher AUC than single measurements.

\section{Conclusion}

Longitudinal biomarker change between 12 and 30 weeks could improve prediction of EO-PD compared to single measurements.

\section{KEYWORDS}

Early Pregnancy Complications, Eclampsia, HELLP, High-Risk Pregnancy, Pregnancy induced Hypertension, Placenta, Preeclampsia, Fetal Growth Restriction

\section{KEY MESSAGE}

In high-risk women, differences in angiogenic biomarkers PlGF, sFlt-1 and more importantly sFlt-1/PlGF ratio were found in early onset placental dysfunction, but not in late-onset placental dysfunction. Longitudinal change in these biomarkers between 12-30 weeks marginally improved the prediction of early onset placental dysfunction compared to measurement at a single time point. 


\section{INTRODUCTION}

The phenotypes of placental dysfunction, especially those affected by defective deep placentation, are significantly related to maternal and perinatal mortality and morbidity [1, 2, 3-8]. Adequate screening to identify pregnant women at risk of developing placental dysfunction could help improve the efficacy and quality of antenatal care, thus improving maternal and perinatal outcomes [9-11]. Earlyonset placental dysfunction is believed to arise from inadequate trophoblastic invasion, that leads to insufficient remodeling of the spiral arteries affecting placental perfusion. This placental malperfusion is associated with a relative decrease in angiogenesis-related factors (e.g. Placental growth factor (PlGF)) and a relative increase in anti-angiogenesis factors (e.g. soluble fms-like tyrosine kinase 1 (sFlt-1)) [12]. Placental growth factor (PIGF) is a pro-angiogenic factor predominantly expressed in placental trophoblast and binds to VEGF-receptor or fms-like tyrosine-1 (Flt1), thereby enhancing the pro-angiogenic action of VEGF-A. Besides this membrane-bound receptor form, Flt- 1 also exists in a soluble form (sFlt-1), which is mainly secreted by the placenta and scavenges VEGF and PlGF, thereby lowering circulating levels of unbound VEGF and PIGF $[13,14,15]$. The placenta is the major source of sFlt- 1 during pregnancy, however in non-pregnant women, sFlt- 1 is also produced in small amount by endothelial cells and monocytes [16]. Although the exact source of increased sFlt- 1 in the circulation is not yet determined, rapid decline in sFlt1 levels after delivery and the upregulation of placental sFlt1 mRNA strongly suggest a placental origin of the sFlt-1 [17].

Late-onset preeclampsia is thought to be associated with trophoblast dysfunction due to villous overcrowding in term placentas, leading to diminished intervillous perfusion and increased hypoxia [18]. It seems that the placenta outgrows the capacity of uterine vasculature to support the increasing demands of the term placenta [19-22]. Another theory is that placental dysfunction, mainly in late onset disease, is an epiphenomenon caused by failure of the maternal cardiovascular system to adapt to pregnancy [23]. There is an increase in cardiovascular load on the maternal heart and vasculature throughout pregnancy, with an accompanying increase in shear stress and endothelial activation. Late-onset preeclampsia has been linked to preexisting maternal conditions that could affect endothelial integrity by a sytematic maternal inflammatory response, consequently resulting in endothelial dysfunction [24-26].

Many obstetric syndromes have been associated with defective deep placentation. Most studies investigated the relation of serum level sFlt-1 and PIGF in maternal placental syndrome, mostly preeclampsia. Few studies looked at both maternal and fetal complications [27-28]. Previous studies of these biomarkers 
demonstrated no significant difference in the maternal blood level of sFlt-1 between preeclampsia and control pregnancies until the second half of pregnancy, whereas PIGF already differed substantially in the first trimester [29-32]. Only a few studies have evaluated longitudinal changes during the different trimesters in sFlt- 1 and PIGF and the results are variable [27, 28, 32-36] and could be explained by different subtypes of placental dysfunction.

Many experts consider onset of placental dysfunction before 34 weeks as an indicator of a severe subtype related to placental bed pathology, while onset after 34 weeks has usually a milder course and is more related to placental capacity [22, 37]. Angiogenesis-related biomarkers could possibly discriminate between these subtypes of placental dysfunction. However there is a lack of literature on longitudinal changes in biomarker levels during pregnancy in early versus lateonset placental dysfunction.

The first objective of this study was to assess whether the subtypes of placental dysfunction (early versus late-onset) are associated with a change in Placental Growth Factor (PIGF) and soluble Fms-like tyrosine kinase-1 (sFlt-1) levels. And second to assess whether the performance of screening by these biomarkers is improved by calculating the longitudinal biomarker changes compared to single timepoint biomarker measurements.

\section{MATERIALS AND METHODS}

\section{Study design and participants}

We performed a retrospective cohort study in women with singleton pregnancies between January 2015 and December 2017. Participants were recruited from a monitoring program at the department of obstetrics at the Maastricht University Medical Centre, where high risk women structurally undergo cardiovascular measurements at 12, 16, 20 and 30 weeks of pregnancy. High risk was defined as having pre-existent hypertension, diabetes mellitus, an auto-immune disease or a previous pregnancy complicated by hypertension, preeclampsia, HELLPsyndrome, eclampsia and/or related fetal complications including delivery of a small for gestational age neonate, stillbirth or placental abruption. All women with singleton pregnancies at high risk of developing placental dysfunction based on obstetric history and/or medical history were included. We excluded 154 pregnant women, who had missed more than two visits (at 12,16, 20 or 30 weeks) $(\mathrm{n}=149)$, had multiple pregnancy $(\mathrm{n}=4)$ or multiple congenital disorders $(\mathrm{n}=1)$.

Relevant maternal baseline characteristics, like date of birth, method of conception, cigarette smoking during pregnancy, medical and obstetric history 
including parity and obstetric complications were recorded. Patients who refused using their records, were excluded in this study. The study protocol was approved by the medical ethical committee of the Maastricht University Medical Centre (17-4-0.15.1/ab). All procedures were in accordance with institutional guidelines and adhered to the principles of the Declaration of Helsinki and Title 45, U.S. Code of Federal Regulations, Part 46, Protection of Human Subjects (revised 13 November 2001, effective 13 December 2001).

\section{Placental dysfunction}

Early-onset placental dysfunction was defined as placenta-mediated complications diagnosed before 34 weeks: early-onset fetal growth restriction (FGR), early-onset preeclampsia/eclampsia, preeclampsia in combination with HELLP syndrome (hemolysis, elevated liver enzyme levels, and low platelet levels) and placental abruption. Late-onset placental dysfunction was defined as preeclampsia or pregnancy induced hypertension (PIH), diagnosed after 34 weeks of gestation.

Preeclampsia (PE) was defined according to the guidelines of International society for the Study of Hypertension in Pregnancy [38]. In normotensive women at baseline, PE was defined as the development of hypertension with proteinuria. When a woman was suspected of developing hypertension, blood pressure was recorded for a period of $30 \mathrm{~min}$ at 3-min intervals using a semiautomatic oscillometric device in half-sitting position. Median values of 9 subsequent recordings were used for analysis. Hypertension was then defined as a median systolic blood pressure equal to or greater than $140 \mathrm{mmHg}$, and/or a diastolic blood pressure equal or greater than $90 \mathrm{mmHg}$. The diagnosis of proteinuria required excretion of albumin/creatinine ratio of more than $30 \mathrm{mg} / \mathrm{mmol}$, without evidence of a urinary tract infection. Chronic hypertension was defined by documentation of hypertension and/or required antihypertensive-drug therapy by medical records. In patients with chronic hypertension, $\mathrm{PE}$ was defined as new-onset proteinuria after gestation. FGR was defined in accordance with the national standard, as an abdominal circumference $(\mathrm{AC})$, or an estimated fetal weight $<10$ th percentile, measured during routine ultrasound scans [39].

\section{Angiogenesis-related biomarkers}

Blood samples were analyzed in a certified laboratory using commercially available kits by personnel who were unaware of the outcome of the pregnancy. Measurements of sFlt-1 and PIGF assays were performed according to the manufacturer's instructions on the fully automated BRAHMS KRYPTOR compact PLUS system (ThermoFisher Scientific, Hennigsdorf, Germany). Both assays are homogeneous sandwich immunoassays based on the Time Resolved Amplified Cryptate 
Emission (TRACE) technology. The PlGF assay has an analytical measuring range of $6.7-7000 \mathrm{pg} / \mathrm{ml}$ and the sFlt- 1 assay has an analytical measuring range of 29$90.000 \mathrm{pg} / \mathrm{mL}$.

The limit of detection (LOD) is $3.6 \mathrm{pg} / \mathrm{ml}$ and $22 \mathrm{pg} / \mathrm{ml}$ for PlGF and sFlt-1, respectively. The functional assay sensitivity (FAS) (inter-assay precision of $20 \%$ ) is $6.7 \mathrm{pg} / \mathrm{ml}$ and $29 \mathrm{pg} / \mathrm{ml}$ for PlGF and sFlt-1, respectively. The limit of quantitation (with total error (imprecision and bias) at $40 \%$ ) is $6.9 \mathrm{pg} / \mathrm{ml}$ and $34 \mathrm{pg} / \mathrm{ml}$ for PlGF and sFlt-1, respectively. The LOD and LOQ have been determined following CLSI EP17-A guideline (Protocols for Determination of Limits of Detection and Limits of Quantitation). The FAS have been determined following CLSI EP05-A2 guideline (Evaluation of Precision Performance of Quantitative Measurement Methods). Measurements were performed in singleton. Quality control samples were included in each run. Total precision for both assays displayed CVs of $<10 \%$.

All patients had serial measurements of PlGF and sFlt-1 and the corresponding ratio was calculated. We evaluated the longitudinal change in serum concentrations of sFlt-1, PlGF and sFlt-1/PlGF ratio at different intervals (12-16, 12-20, 12-30, 16-20, 16-30, and 20-30 weeks gestation).

\section{Statistical methods}

Normally distributed baseline characteristics are presented as mean with standard deviation, not normally distributed data are presented as median. Categorical variables are depicted as percentages $(n / N)$. Maternal baseline characteristics were compared between groups using analysis of variance (ANOVA) or Kruskal-Wallis test for continuous variables and Fisher's exact test or chi-square test for categorical variables. An unpaired $t$-test was used to analyses differences between groups with normally distributed data. For non-normal distributed data, we used the Mann-Whitney U test.

We tested differences in serum biomarkers levels at the different time intervals between the reference group and each outcome group separately: early-onset placental dysfunction and subdividing the late-onset placental dysfunction group in late-onset PE and PIH, with one way-ANOVA or the Kruskal-Wallis test.

Repeated-measures ANOVA was used to examine differences in biomarker levels between reference group and early-onset placental dysfunction, late-onset $\mathrm{PE}, \mathrm{PIH}$ and over the multiple time points, to assess the effects of gestational age, and to determine if the course of biomarker levels over the multiple time points differs between placental dysfunction and reference group (i.e., whether an interaction between gestational age and group is present).

For the second aim the longitudinal change was calculated as the second measurement divided by the value of a measurement at an earlier gestational age. 
This value was expressed as a longitudinal change (\%) compared with the earlier measurement. For example, to calculate the percentage of change in serum level compared to an earlier measurement of PIGF between 12 and 30 weeks: ((PIGF at 30 weeks / PlGF at 12 weeks) *100).

The performance of detecting placental dysfunction by PlGF, sFlt-1 and sFlt-1/ PIGF ratio by single measurements at 12,16, 20 and 30 weeks, as well as the longitudinal change was determined by a univariate analysis of the (delta) biomarker with a binary logistic regression followed by a receiver operator characteristic (ROC) curve analysis. The area under the curves (AUCs) were used to assess the ability to discriminate between placental dysfunction and control group. IBM SPSS 23 (SPSS Inc. Chicago, IL, USA) was used for statistical analysis.

\section{RESULTS}

\section{Different outcome groups}

Of a total of 207 singleton high-risk pregnancies, 133 had uncomplicated pregnancy outcomes (reference group) (64\%), 43 developed early-onset placental dysfunction (21\%), and 31 developed a late-onset placental dysfunction (15\%). The early-onset placental dysfunction group consisted of women with severe earlyonset normotensive fetal growth restriction ( $\mathrm{n}=28$ ), severe early-onset PE (before 34 weeks) ( $n=9)$, severe early-onset PE with fetal growth restriction $(n=4)$ and placental abruption ( $\mathrm{n}=2$ ), no women developed eclampsia or HELLP syndrome. The late-onset placental dysfunction group consisted of 16 patients who developed late-onset PE (after 34 weeks) (7.7\%), none of them developed FGR, and 15 patients who developed pregnancy induced hypertension (PIH, 7.2\%).

Maternal and fetal characteristics for each outcome group are summarized in Table 1 . There were no significant differences between the groups in baseline characteristics, except in mean maternal height and auto-immune diseases. Early-onset placental dysfunction and late-onset PE groups had significant lower birthweight and a shorter gestational age at delivery compared with the reference group.

\section{PIGF, sFlt-1 and sFlt-1/PIGF ratio at 12, 16, 20 and 30 weeks of gestation}

Maternal serum levels of PIGF and sFlt-1 were determined in 757 samples from 207 patients. The maternal serum values of PlGF, sFlt-1 and sFLt-1/PIGF ratio were compared between each different outcome groups (early-onset placental dysfunction, late-onset PE, and PIH) and the reference group at 12, 16, 20 or 30 weeks of gestation (Table 2). Mean maternal PIGF values were significantly lower 


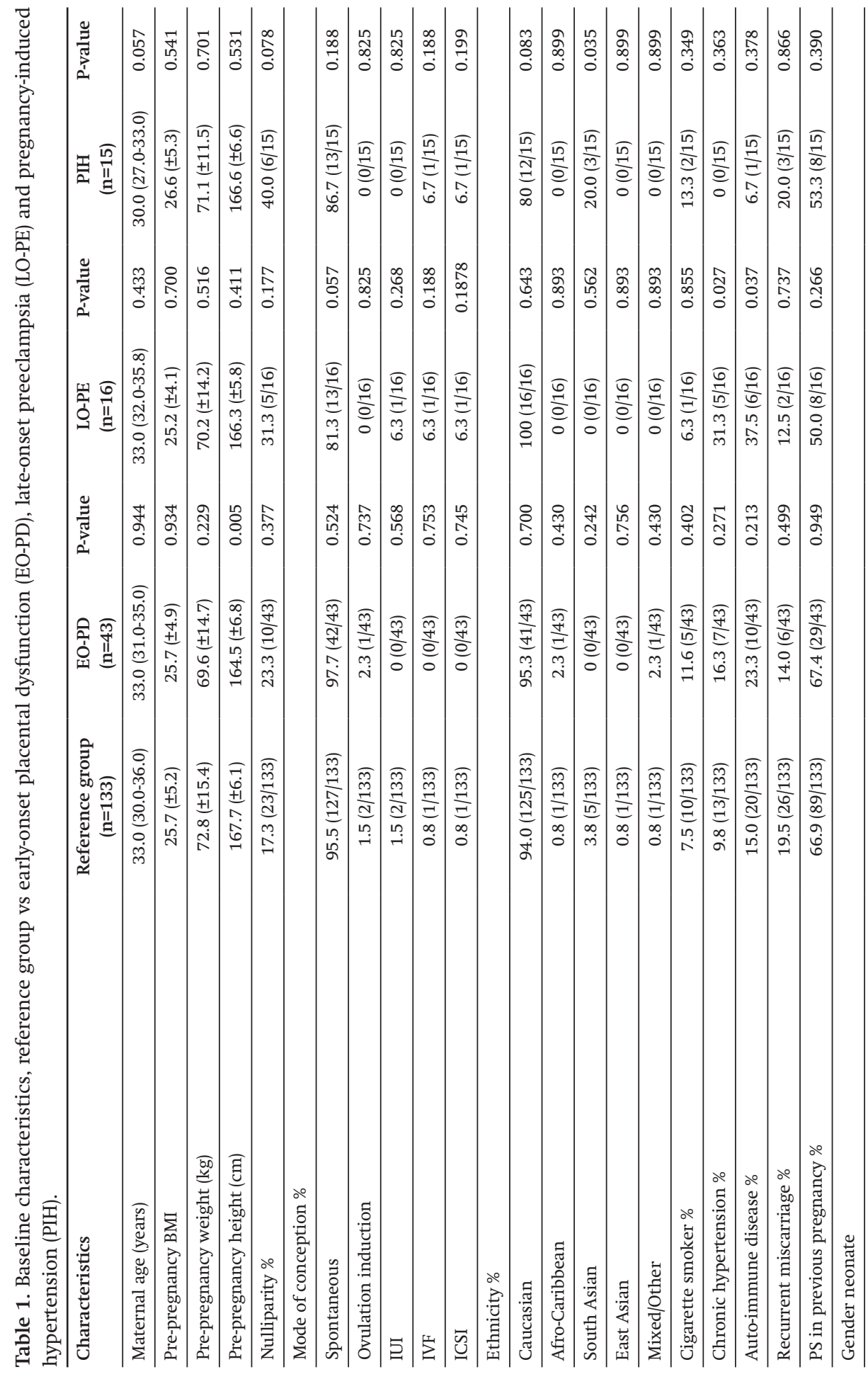




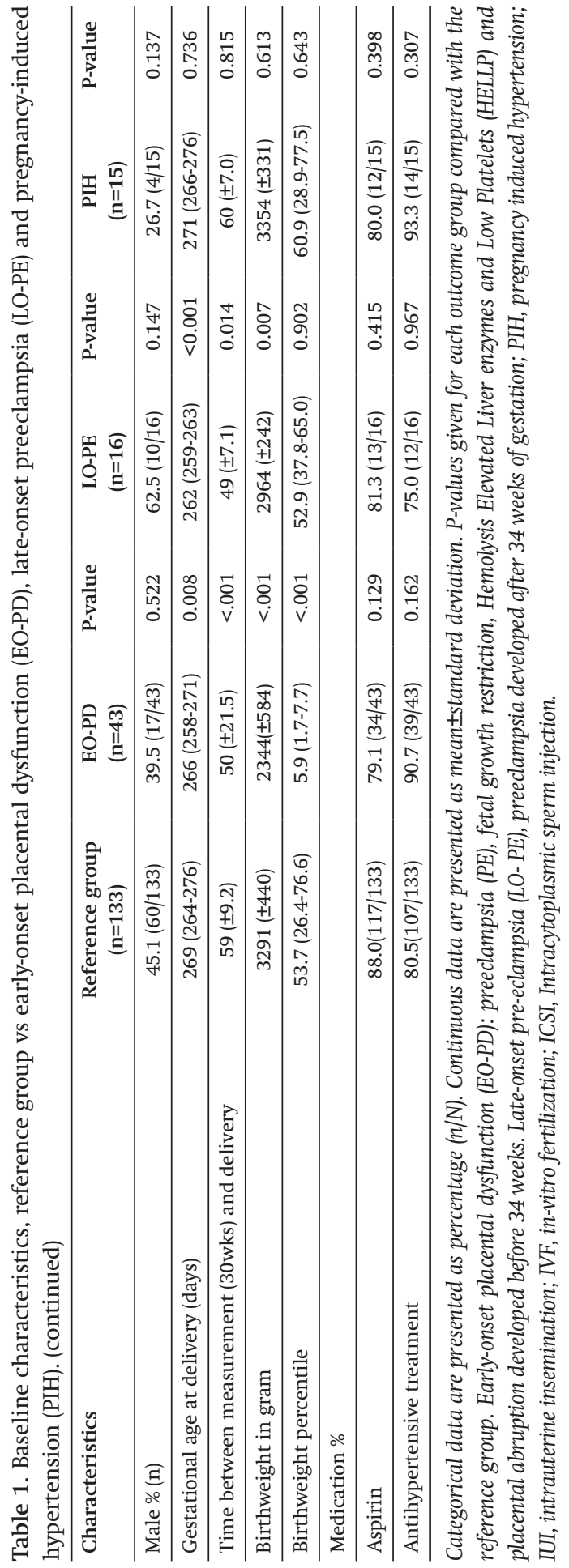


Table 2. Biomarkers, reference group vs early-onset placental dysfunction (EO-PD), late-onset pre-eclampsia (LO-PE) and pregnancy induced hypertension (PIH) PlGF, sFlt-1 and the ratio at 12, 16, 20 and 30 weeks.

\begin{tabular}{|c|c|c|c|c|c|c|c|}
\hline PlGF (pg/ml) & $12 w k s$ & $16 w k s$ & $20 w k s$ & $30 w k s$ & Group & Time & $\begin{array}{c}\text { Group } \\
\mathbf{x} \\
\text { Time }\end{array}$ \\
\hline Reference & $30.1 \pm 11.09$ & $76.5 \pm 91.91$ & $149.4 \pm 69.83$ & $400.2 \pm 224.55$ & \multirow{3}{*}{$<0.001$} & \multirow{3}{*}{$<0.001$} & \multirow{3}{*}{$<0.001$} \\
\hline EO-PD & $33.3 \pm 17.87$ & $74.4 \pm 34.22$ & $140.0 \pm 76.21$ & $256.6 \pm 142.30$ & & & \\
\hline P-value & 0.229 & 0.738 & 0.459 & $<0.001$ & & & \\
\hline LO-PE & $31.7 \pm 14.77$ & $87.7 \pm 37.59$ & $167.5 \pm 71.11$ & $413.7 \pm 286.64$ & \multirow{2}{*}{0.514} & \multirow{2}{*}{$<0.001$} & \multirow{2}{*}{0.989} \\
\hline P-value & 0.684 & 0.216 & 0.372 & 0.827 & & & \\
\hline PIH & $32.1 \pm 14.55$ & $78.8 \pm 34.72$ & $161.0 \pm 78.81$ & $439.3 \pm 265.01$ & \multirow{2}{*}{0.129} & \multirow{2}{*}{$<0.001$} & \multirow{2}{*}{0.036} \\
\hline P-value & 0.626 & 0.805 & 0.553 & 0.516 & & & \\
\hline \multicolumn{8}{|l|}{ sFlt-1 (pg/ml) } \\
\hline Reference & $1128 \pm 482.6$ & $1158 \pm 571.7$ & $1139 \pm 579.8$ & $1459 \pm 881.7$ & \multirow{3}{*}{$<0.001$} & \multirow{3}{*}{$<0.001$} & \multirow{3}{*}{0.088} \\
\hline EO-PD & $1195 \pm 503.2$ & $1270 \pm 614.4$ & $1454 \pm 1142.8$ & $1993 \pm 1607.6$ & & & \\
\hline P-value & 0.494 & 0.309 & 0.022 & 0.013 & & & \\
\hline LO-PE & $1362 \pm 564.4$ & $1322 \pm 552.8$ & $1315 \pm 824.6$ & $1896 \pm 1233.1$ & \multirow{2}{*}{0.007} & \multirow{2}{*}{0.001} & \multirow{2}{*}{0.712} \\
\hline P-value & 0.100 & 0.320 & 0.420 & 0.176 & & & \\
\hline $\mathrm{PIH}$ & $1207 \pm 587.2$ & $1242 \pm 845.1$ & $1300 \pm 898.2$ & $1626 \pm 1465.1$ & \multirow{2}{*}{0.569} & \multirow{2}{*}{0.013} & \multirow{2}{*}{0.989} \\
\hline P-value & 0.601 & 0.620 & 0.444 & 0.593 & & & \\
\hline \multicolumn{8}{|l|}{ sFlt-1/PlGF } \\
\hline Reference & $41.7 \pm 23.75$ & $17.5 \pm 12.81$ & $8.9 \pm 5.81$ & $6.7 \pm 14.77$ & \multirow{3}{*}{0.001} & \multirow{3}{*}{$<0.001$} & \multirow{3}{*}{0.004} \\
\hline EO-PD & $46.9 \pm 35.14$ & $30.6 \pm 58.30$ & $29.3 \pm 118.19$ & $16.6 \pm 29.90$ & & & \\
\hline P-value & 0.309 & 0.012 & 0.036 & 0.006 & & & \\
\hline LO-PE & $49.4 \pm 25.06$ & $19.1 \pm 15.55$ & $9.0 \pm 5.98$ & $9.7 \pm 10.90$ & \multirow{2}{*}{0.146} & \multirow{2}{*}{$<0.001$} & \multirow{2}{*}{0.627} \\
\hline P-value & 0.295 & 0.833 & 0.994 & 0.579 & & & \\
\hline $\mathrm{PIH}$ & $40.3 \pm 17.14$ & $18.3 \pm 14.45$ & $9.9 \pm 9.04$ & $20.5 \pm 19.31$ & \multirow{2}{*}{0.297} & \multirow{2}{*}{$<0.001$} & \multirow{2}{*}{0.957} \\
\hline P-value & 0.866 & 0.918 & 0.948 & 0.672 & & & \\
\hline
\end{tabular}

Continuous data are presented as mean \pm standard deviation. P-values given for each outcome group compared with the reference group. Early-onset placental dysfunction (EO-PD): preeclampsia (PE), fetal growth restriction, Hemolysis Elevated Liver enzymes and Low Platelets (HELLP) and placental abruption developed before 34 weeks. Late-onset pre-eclampsia (LO- PE), preeclampsia developed after 34 weeks of gestation; PIH, pregnancy induced hypertension; Repeated measures ANOVA: Group: EO-PD, LO-PE or PIH vs reference group. Time: 12, 16, 20 and 30 weeks of gestational age. Group x time: interaction between groups and gestational age. PlGF, placental growth factor; sFlt-1, soluble fms-like tyrosine kinase 1.

in the early-onset placental dysfunction group compared to the reference group at 30 weeks $(256.6 \pm 142.30 \mathrm{pg} / \mathrm{ml}$ vs $400.2 \pm 224.55, \mathrm{p}=<0.001)$, but not at 12,16 and 20 weeks. No significant differences in PlGF values were found between the PIH group and late-onset PE group versus the reference group at any time point. Mean maternal sFlt-1 values were higher in the early-onset placental dysfunction, compared to the reference group, both at 20 weeks $(1454 \pm 1142.8 \mathrm{pg} / \mathrm{ml}$ vs 
Table 3. Longitudinal change of PlGF, sFlt-1 and the sFlt-1/PlGF ratio.

\begin{tabular}{|c|c|c|c|c|c|c|c|}
\hline PlGF (pg/ml) & Reference & EO-PD & P-value & LO-PE & P-value & $\mathrm{PIH}$ & P-value \\
\hline $12-16$ & $161 \% \pm 99.3$ & $142 \% \pm 102.2$ & 0.320 & $180 \% \pm 74.3$ & 0.491 & $142 \% \pm 61.8$ & 0.508 \\
\hline $12-20$ & $423 \% \pm 224.6$ & $386 \% \pm 388.4$ & 0.461 & $485 \% \pm 126.7$ & 0.414 & $357 \% \pm 135.7$ & 0.400 \\
\hline $12-30$ & $1268 \% \pm 718.7$ & $828 \% \pm 629.8$ & 0.003 & $1128 \% \pm 675.1$ & 0.517 & $1329 \% \pm 803.2$ & 0.779 \\
\hline $16-20$ & $104 \% \pm 64.1$ & $101 \% \pm 72.1$ & 0.780 & $115 \% \pm 49.6$ & 0.581 & $99 \% \pm 42.0$ & 0.758 \\
\hline $16-30$ & $441 \% \pm 299.8$ & $276 \% \pm 214.3$ & 0.002 & $214 \% \pm 219.8$ & 0.138 & $472 \% \pm 227.5$ & 0.692 \\
\hline $20-30$ & $169 \% \pm 120.5$ & $85 \% \pm 80.9$ & $<0.001$ & $136 \% \pm 142.1$ & 0.344 & $183 \% \pm 119.3$ & 0.667 \\
\hline \multicolumn{8}{|l|}{ sFlt-1 (pg/ml) } \\
\hline $12-16$ & $2 \% \pm 29.8$ & $12 \% \pm 31.8$ & 0.132 & $-1 \% \pm 39.1$ & 0.720 & $5 \% \pm 30.2$ & 0.769 \\
\hline $12-20$ & $2 \% \pm 37.6$ & $31 \% \pm 89.7$ & 0.011 & $8 \% \pm 88.2$ & 0.730 & $9 \% \pm 43.0$ & 0.714 \\
\hline $12-30$ & $39 \% \pm 77.4$ & $83 \% \pm 167.8$ & 0.049 & $59 \% \pm 155.7$ & 0.530 & $42 \% \pm 72.6$ & 0.928 \\
\hline $16-20$ & $0.4 \% \pm 20.6$ & $10 \% \pm 31.7$ & 0.030 & $-0.3 \% \pm 28.0$ & 0.915 & $5 \% \pm 17.5$ & 0.475 \\
\hline $16-30$ & $35 \% \pm 62.5$ & $65 \% \pm 88.6$ & 0.020 & $39 \% \pm 67.9$ & 0.821 & $32 \% \pm 42.5$ & 0.880 \\
\hline $20-30$ & $38 \% \pm 92.1$ & $67 \% \pm 119.6$ & 0.100 & $35 \% \pm 44.3$ & 0.920 & $23 \% \pm 41.1$ & 0.567 \\
\hline \multicolumn{8}{|l|}{ sFlt-1/P1GF } \\
\hline $12-16$ & $-56 \% \pm 22.4$ & $-46 \% \pm 33.2$ & 0.062 & $-63 \% \pm 13.8$ & 0.258 & $-53 \% \pm 19.7$ & 0.753 \\
\hline $12-20$ & $-77 \% \pm 15.4$ & $-58 \% \pm 76.5$ & 0.011 & $-81 \% \pm 13.7$ & 0.715 & $-74 \% \pm 14.5$ & 0.755 \\
\hline $12-30$ & $-83 \% \pm 30.8$ & $-69 \% \pm 79.9$ & 0.032 & $-82 \% \pm 15.5$ & 0.955 & $-81 \% \pm 30.8$ & 0.911 \\
\hline $16-20$ & $-46 \% \pm 22.0$ & $-39 \% \pm 30.3$ & 0.111 & $-51 \% \pm 18.8$ & 0.510 & $-45 \% \pm 13.2$ & 0.891 \\
\hline $16-30$ & $-62 \% \pm 60.6$ & $-27 \% \pm 100.4$ & 0.008 & $-53 \% \pm 34.4$ & 0.680 & $-69 \% \pm 31.9$ & 0.722 \\
\hline $20-30$ & $-25 \% \pm 113.0$ & $59 \% \pm 271.0$ & 0.004 & $-7 \% \pm 74.4$ & 0.704 & $-40 \% \pm 50.2$ & 0.735 \\
\hline
\end{tabular}

Longitudinal change is presented as percentage (\%) Istandard deviation. P-values given for each outcome group compared with the reference group. Early-onset placental dysfunction (EO-PD): developed before 34 weeks. Late-onset preeclampsia (LO- PE); PIH, pregnancy induced hypertension; PlGF=placental growth factor. sFlt$1=$ soluble fms-like tyrosine kinase 1.

$1139 \pm 579.8 \mathrm{pg} / \mathrm{ml}, \mathrm{p}=0.022)$, and at 30 weeks of gestation $(1993 \pm 1607.6 \mathrm{pg} / \mathrm{ml}$ vs $1459 \pm 881.7 \mathrm{pg} / \mathrm{ml}, \mathrm{p}=0.013)$. No significant differences in sFlt-1 values were found between the PIH group and late-onset PE group versus the reference group at any time point. Similarly, the sFlt-1/PlGF ratio was significantly higher in the early-onset placental dysfunction group compared to the reference group at 16 weeks ( $30.6 \pm 58.30$ vs $17.5 \pm 12.81, \mathrm{p}=0.012)$, at 20 weeks $(29.3 \pm 118.19$ vs $8.9 \pm 5.8$, $\mathrm{p}=0.036)$, and at 30 weeks ( $16.6 \pm 29.90$ vs $6.7 \pm 14.77, \mathrm{p}=0.006)$.

The effect of time was different between the early-onset placental dysfunction, late-onset PE and PIH versus reference. The interaction effect of group * time was statistically significant in PlGF for early-onset placental dysfunction $(\mathrm{p}<0.001)$ and PIH ( $\mathrm{p}=0.036)$ versus the reference group. The interaction effect was also significant in the sFlt-1/PlGF ratio for early-onset placental dysfunction $(\mathrm{p}=0.004)$ versus reference group. 


\section{Longitudinal changes in PlGF, sFlt-1 and sFlt-1/PlGF ratio}

The longitudinal changes between PlGF, sFlt-1 and the ratio among the four different outcome groups are displayed in Table 3. Patients who developed early-onset placental dysfunction, compared to the reference group, had a lower increase in PlGF (\%) at 12-30 ( $\mathrm{p}=0.003), 16-30(\mathrm{p}=0.002)$, and 20-30 weeks interval $(\mathrm{p}<0.001)$, and a higher increase in sFlt- $1(\%)$ at $12-20(\mathrm{p}=0.011), 12-30$ weeks $(\mathrm{p}=0.049), 16-20$ $(\mathrm{p}=0.030)$ and at $16-30$ weeks $(\mathrm{p}=0.020)$. Similarly, the $\mathrm{sFlt}-1 / \mathrm{PlGF}$ ratio showed less decrease in the EO-PD group vs reference group at $12-20(\mathrm{p}=0.011), 12-30$ $(\mathrm{p}=0.032), 16-30 \quad(\mathrm{p}=0.008)$ and the 20-30 weeks interval $(\mathrm{p}=0.004)$.

No significant differences in longitudinal values of biomarkers were detected for the late-onset PE or the PIH vs reference group at any time interval.

\section{PIGF, sFlt-1 and sFlt-1/PIGF ratio in predicting early-onset placental dysfunction}

The sFlt-1/PIGF ratio had a better performance for the prediction of early-onset placental dysfunction (Table 4) than either sFlt-1 or PlGF alone (data not shown). For single time point measurements the diagnostic differentiation (AUC) increased with gestational age from $0.51(0.19-0.62)$ at 12 weeks to $0.7(0.60-0.78)$ at 30 weeks. The longitudinal change of sFlt-1/PIGF ratio for all intervals starting from 12, 16 or 20 weeks had higher AUC than single measurements at 12, 16 or 20 weeks, respectively. No difference in AUC was seen between the single measurement at 30 weeks and the longitudinal change for all intervals ending with 30 weeks (all around 0.7).

Table 4. Performance of screening for early onset placental dysfunction in high risk population by the sFlt-1/PIGF ratio.

\begin{tabular}{llc}
\hline $\begin{array}{l}\text { Screening measurement sFlt-1/PIGF } \\
\text { ratio for placental dysfunction }\end{array}$ & AUC $(95 \%$ CI $)$ & $\begin{array}{c}\text { Odds ratio } \\
\text { logistic regression }\end{array}$ \\
\hline 12 weeks & $0.51(0.19-0.62)$ & $1.006(0.993-1.019)$ \\
\hline 16 weeks & $0.55(0.44-0.65)$ & $1.016(0.998-1.035)$ \\
\hline 20 weeks & $0.61(0.52-0.71)$ & $1.050(1.001-1.101)$ \\
\hline 30 weeks & $0.69(0.60-0.78)$ & $1.020(1.003-1.038)$ \\
\hline Relative change $12-16$ & $0.63(0.53-0.73)$ & $3.875(0.933-16.085)$ \\
\hline Relative change $12-20$ & $0.71(0.62-0.80)$ & $10.002(0.930-107.631)$ \\
\hline Relative change $12-30$ & $0.70(0.61-0.80)$ & $2.213(0.884-5.542)$ \\
\hline Relative change $16-20$ & $0.60(0.50-0.70)$ & $3.144(0.788-12.548)$ \\
\hline Relative change $16-30$ & $0.70(0.61-0.79)$ & $1.771(1.083-2.897)$ \\
\hline Relative change $20-30$ & $0.69(0.60-0.78)$ & $1.344(1.040-1.737)$ \\
\hline
\end{tabular}

Data are given as area under the curve (AUC) with 95\% confidence interval (CI); ROC, receiver operating characteristics; PlGF, placental growth factor; sFlt-1=soluble fms-like tyrosine kinase 1. 


\section{DISCUSSION}

\section{Interpretation of main findings}

This study demonstrated that at 30 weeks of gestation PIGF and sFlt- 1 are both significantly different among patients who developed early-onset placental dysfunction when compared to the reference group. Earlier in pregnancy, at 16 weeks, the sFlt-1/PIGF ratio was already significantly higher in the early-onset placental dysfunction versus the reference group. In the early-onset placental dysfunction group, PIGF had a significantly lower increase during gestation and sFlt-1 has a significantly higher increase when compared to the reference group. This is in agreement with previous studies who found that a lower increase in maternal serum PIGF is a risk factor for the subsequent development of placental dysfunction $[27,34,40]$. The difference between PIGF in the early-onset placental dysfunction and the reference group increases with gestation, which is also consistent with earlier studies [27, 28, 32-34, 40]. The interaction effect of group and time was tested and showed that both have a significant influence on the PIGF and sFlt-1/ PlGF values for early-onset placental dysfunction during pregnancy.

These study results also confirm that not only early-onset PE, but also FGR and placental abruption are related to an imbalance in sFlt-1 and PIGF [41]. Both earlyonset placental dysfunction disorders may have the same underlying pathology from inadequate trophoblastic invasion, that leads to insufficient remodeling of the spiral arteries, resulting in placental under perfusion and ischemia. This causes short-time maternal and fetal consequences, and at a longer period of time possibly a higher risk on cardiovascular disease [37].

Furthermore, this study demonstrated that patients who developed late-onset PE after 34 weeks of gestation did not show any significant differences in PlGF and sFlt-1, when compared to the reference group. It reflects a heterogenous condition with minimal placental involvement. This is in line with our earlier hypothesis that late-onset placental dysfunction is thought to be associated with trophoblast dysfunction due to villous overcrowding in term placentas, leading to diminished intervillous perfusion and increased hypoxia. Later in pregnancy, especially at term, the increased placenta demands more than the uterine vasculature is able to provide [25].

\section{Longitudinal change}

The second objective was to assess whether the performance of screening by these biomarkers is improved by calculating the longitudinal biomarker changes instead of single timepoint biomarker measurements. Several studies investigating the longitudinal changes in maternal serum sFlt-1 and PIGF levels found that 
longitudinal measurements form better predictors than measurements at a single point during pregnancy [28, 32, 34]. This study confirmed that a single meaurement of sFlt-1 and PIGF in the first or early second trimester is a poor predictor of developing early onset placental dysfunction. However the overall performance could be improved by repeated measurements [28, 32]. Women with early-onset placental dysfunction, compared to the reference group, had a lower increase in PlGF and a higher increase in sFlt-1 at 12-30, 16-30 and 20-30 weeks. Similarly, the sFlt-1/PIGF ratio showed less decrease in the early-onset placental dysfunction group versus reference group at 12-20, 12-30, 16-30 and the 20-30 weeks interval. These differences in longitudinal values were not seen in the late-onset placental dysfunction group, confirming the different underlying pathology.

The sFlt-1/PIGF ratio had a better performance for the prediction of early-onset placental dysfunction than either sFlt-1 or PIGF alone. For single time point measurements the diagnostic differentiation (AUC) increased with gestational age from $0.51(0.19-0.62)$ at 12 weeks to $0.7(0.60-0.78)$ at 30 weeks. The longitudinal change of sFlt-1/P1GF ratio for all intervals starting from 12, 16 or 20 weeks had higher AUC than single measurements at 12, 16 or 20 weeks, respectively.

Our results show that the interval between the last blood sampling (all around 30 weeks) and delivery was different between the groups, but it is merely a reflection of the difference in gestational age at delivery. Hence, we argue that the measurement-delivery time interval has little influence on the biomarker values in this study.

There is no consensus on the ideal time point in pregnancy to perform biochemical screening for late placental dysfunction. Measurement at 36 weeks would have a better performance for the prediction of late preeclampsia, necessitating delivery after 37 weeks, compared with 30 weeks [42]. Screening for late FGR using the same panel of biomarkers was feasible at 36 weeks of gestation but performed worse that screening for late preeclampsia [43]. It is possible that late-onset preeclampsia has a milder placental dysfunction at 30 weeks, which leads to smaller changes in biomarker levels. However, a more tempting theory is that late onset preeclampsia has a different pathophysiology to early onset disease, and that this is reflected on the maternal serum biomarker profile. We speculate that, as the vascular entity leading to late onset preeclampsia worsens towards the end of pregnancy, a disbalance in biomarker ratio occurs which resembles that of early onset preeclampsia, albeit different in its source, severity and rate of development. This is supported by that fact that in both early- and late-onset subtypes, the closer the measurement is to the clinical presentation (or the indicated delivery), the more deranged the biomarker is [44]. 


\section{High risk population}

A high-risk population was selected to screen for different placental dysfunction profiles. The incidence of placental dysfunction in this study population is $20.8 \%$. Also, because patients were included in this high-risk monitoring program, as described earlier, the gestational age between the patients at 12, 16, 20 and 30 weeks were similar and found to be not significantly different. Hence, no additional measures were needed in order to 'normalize' the data. Many studies have focused on low-risk populations, but only few have evaluated the use of sFlt-1 and PlGF in a high-risk population [33, 40, 45, 46]. In this study we observed that PlGF was lower and sFlt-1 and sFlt-1/PlGF rate were higher in patients who develop placental dysfunction in comparison to the reference group. These findings, although not significant, are similar to that previously reported among low-risk patients [27, 28, 30, 32-36, 45-48]. This suggests that the pathogenesis of placental dysfunction in both low and high-risk women are similar.

The majority of the studied population used aspirin. It is hypothesized that aspirin improves the trophoblast cell integration by inhibiting the effects of Tumor Necrosis Factor alpha (TNF- $\alpha$ ), however the effect of aspirin on the concentration of the (anti)angiogenic biomarkers is still unclear. Several studies have shown no significant effect on the concentration of PIGF and sFlt-1 with the use of aspirin $[40,49,50]$. However, an in-vitro study showed that the treatment of aspirin in patients with PE increased the PIGF concentrations [51]. The improvement in serum PlGF concentrations by aspirin might explain the small difference between 12-20 weeks in PlGF in this study. There is no conclusive data on the effect of methyldopa and labetalol on sFlt-1 and PIGF [52, 53, 54].

\section{Strengths and limitations}

One of the strengths of this study is that it looked at different phenotypes of placental dysfunction, whereas most studies have only focused on PE. Even though multiple obstetric syndromes have been proven to be associated with poor placentation and both PE and FGR have previously shown to be associated with an imbalance in pro-angiogenic and anti-angiogenic factors [27, 28, 41, 53]. Another strength of this study is that it examined the changes of these biomarkers during different pregnancy trimesters. One of the limitations of this study is its retrospective cohort design with substantial loss to follow-up, which makes this study susceptible to bias. Another limitation of this study is the small study sample, with a small range in ethnic profile and mode of conception decreasing generalizability. 


\section{CONCLUSION}

This study confirms that in a high-risk population, early and late-onset placental dysfunction differ in their angiogenic profile, suggesting a different underlying mechanism. In the third trimester all individual biomarkers were significantly related to early-onset placental dysfunction, while in the second trimester only the sFlt-1/PIGF ratio values were different. However the longitudinal change of the sFlt-1/PlGF ratio is already discriminatory from the first trimester.

In order to further investigate the use of the longitudinal change to improve the performance of screening, a large prospective longitudinal study design is required to assess the likelihood of early placental dysfunction based on longitudinal change of sFlt-1, PIGF and the ratio. 


\section{REFERENCES}

1. Brosens I, Pijnenborg R, Vercruysse L, Romero R. The "Great Obstetrical Syndromes" are associated with disorders of deep placentation. Am J Obstet Gynecol. 2011;204(3):193-201.

2. Khong Y, Brosens I. Defective deep placentation. Best Pract Res Clin Obstet Gynaecol. 2011;25(3):301-11.

3. Romero R, Kusanovic JP, Chaiworapongsa T, Hassan SS. Placental bed disorders in preterm labor, preterm PROM, spontaneous abortion and abruptio placentae. Best Pract Res Clin Obstet Gynaecol. 2011;25(3):313-27.

4. Brosens I. Placental bed \& maternal - fetal disorders. Preface. Best Pract Res Clin Obstet Gynaecol. 2011;25(3):247-8.

5. Frusca T, Morassi L, Pecorelli S, Grigolato P, Gastaldi A. Histological features of uteroplacental vessels in normal and hypertensive patients in relation to birthweight. Br J Obstet Gynaecol. 1989;96(7):835-9.

6. Hustin J, Foidart JM, Lambotte R. Maternal vascular lesions in pre-eclampsia and intrauterine growth retardation: light microscopy and immunofluorescence. Placenta. 1983;4 Spec No:489-98.

7. Cantwell R, Clutton-Brock T, Cooper G, Dawson A, Drife J, Garrod D, et al. Saving Mothers' Lives: Reviewing maternal deaths to make motherhood safer: 2006-2008. The Eighth Report of the Confidential Enquiries into Maternal Deaths in the United Kingdom. BJOG. 2011;118 Suppl 1:1-203.

8. Say L, Chou D, Gemmill A, Tuncalp O, Moller AB, Daniels J, et al. Global causes of maternal death: a WHO systematic analysis. Lancet Glob Health. 2014;2(6):e323-33.

9. Bujold E, Roberge S, Lacasse Y, Bureau M, Audibert F, Marcoux S, et al. Prevention of preeclampsia and intrauterine growth restriction with aspirin started in early pregnancy: a meta-analysis. Obstet Gynecol. 2010;116(2 Pt 1):402-14.

10. Villa PM, Marttinen P, Gillberg J, Lokki AI, Majander K, Orden MR, et al. Cluster analysis to estimate the risk of preeclampsia in the high-risk Prediction and Prevention of Preeclampsia and Intrauterine Growth Restriction (PREDO) study. PLoS One. 2017;12(3):e0174399.

11. Frampton GK, Jones J, Rose M, Payne L. Placental growth factor (alone or in combination with soluble fms-like tyrosine kinase 1) as an aid to the assessment of women with suspected pre-eclampsia: systematic review and economic analysis. Health Technol Assess. 2016;20(87):1-160.

12. Groom KM, David AL. The role of aspirin, heparin, and other interventions in the prevention and treatment of fetal growth restriction. Am J Obstet Gynecol. 2018;218(2S):S829S40.

13. Shibuya M. Vascular Endothelial Growth Factor (VEGF) and Its Receptor (VEGFR) Signaling in Angiogenesis: A Crucial Target for Anti- and Pro-Angiogenic Therapies. Genes Cancer. 2011;2(12):1097-105.

14. Chau K, Hennessy A, Makris A. Placental growth factor and pre-eclampsia. J Hum Hypertens. 2017;31(12):782-6.

15. Vrachnis N, Kalampokas E, Sifakis S, Vitoratos N, Kalampokas T, Botsis D, et al. Placental growth factor (PlGF): a key to optimizing fetal growth. J Matern Fetal Neonatal Med. 2013;26(10):995-1002. 
16. Weissgerber TL, Rajakumar A, Myerski AC, Edmunds LR, Powers RW, Roberts JM, et al. Vascular pool of releasable soluble VEGF receptor-1 (sFLT1) in women with previous preeclampsia and uncomplicated pregnancy. J Clin Endocrinol Metab. 2014;99(3):978-87.

17. Maynard SE, Min JY, Merchan J, Lim KH, Li J, Mondal S, et al. Excess placental soluble fmslike tyrosine kinase 1 (sFlt1) may contribute to endothelial dysfunction, hypertension, and proteinuria in preeclampsia. J Clin Invest. 2003;111(5):649-58.

18. Herzog EM, Eggink AJ, Reijnierse A, Kerkhof MA, de Krijger RR, Roks AJ, et al. Impact of early- and late-onset preeclampsia on features of placental and newborn vascular health. Placenta. 2017;49:72-9.

19. Redman CW, Staff AC. Preeclampsia, biomarkers, syncytiotrophoblast stress, and placental capacity. Am J Obstet Gynecol. 2015;213(4 Suppl):S9 e1, S9-11.

20. Pinheiro CC, Rayol P, Gozzani L, Reis LM, Zampieri G, Dias CB, et al. The relationship of angiogenic factors to maternal and neonatal manifestations of early-onset and late-onset preeclampsia. Prenat Diagn. 2014;34(11):1084-92.

21. Zhang X, Jia H, Wang Y, Xie J, Gu Y. [Early and late onset severe preeclampsia: a clinicopathologic study of 178 placentas]. Zhonghua Bing Li Xue Za Zhi. 2015;44(12):879-83.

22. Redman CW. Early and late onset preeclampsia: Two sides of the same coin. 2017.

23. Thilaganathan B. Association of Higher Maternal Blood Pressure With Lower Infant Birthweight: Placental Cause or Cardiovascular Effect? Hypertension. 2016;67(3):499-500.

24. Kalafat E, Thilaganathan B. Cardiovascular origins of preeclampsia. Curr Opin Obstet Gynecol. 2017;29(6):383-9.

25. Ness RB, Roberts JM. Heterogeneous causes constituting the single syndrome of preeclampsia: a hypothesis and its implications. Am J Obstet Gynecol. 1996;175(5):1365-70.

26. Brandao AH, Felix LR, Patricio Edo C, Leite HV, Cabral AC. Difference of endothelial function during pregnancies as a method to predict preeclampsia. Arch Gynecol Obstet. 2014;290(3):471-7.

27. Erez O, Romero R, Espinoza J, Fu W, Todem D, Kusanovic JP, et al. The change in concentrations of angiogenic and anti-angiogenic factors in maternal plasma between the first and second trimesters in risk assessment for the subsequent development of preeclampsia and small-for-gestational age. J Matern Fetal Neonatal Med. 2008;21(5):279-87.

28. Romero R, Nien JK, Espinoza J, Todem D, Fu W, Chung H, et al. A longitudinal study of angiogenic (placental growth factor) and anti-angiogenic (soluble endoglin and soluble vascular endothelial growth factor receptor-1) factors in normal pregnancy and patients destined to develop preeclampsia and deliver a small for gestational age neonate. J Matern Fetal Neonatal Med. 2008;21(1):9-23.

29. Chaiworapongsa T, Romero R, Espinoza J, Bujold E, Mee Kim Y, Goncalves LF, et al. Evidence supporting a role for blockade of the vascular endothelial growth factor system in the pathophysiology of preeclampsia. Young Investigator Award. Am J Obstet Gynecol. 2004;190(6):1541-7; discussion 7-50.

30. Levine RJ, Lam C, Qian C, Yu KF, Maynard SE, Sachs BP, et al. Soluble endoglin and other circulating antiangiogenic factors in preeclampsia. N Engl J Med. 2006;355(10):992-1005.

31. Jaaskelainen T, Heinonen S, Hamalainen E, Pulkki K, Romppanen J, Laivuori H, et al. Angiogenic profile in the Finnish Genetics of Pre-Eclampsia Consortium (FINNPEC) cohort. Pregnancy Hypertens. 2018;14:252-9.

32. Andrietti S, Carlucci S, Wright A, Wright D, Nicolaides KH. Repeat measurements of uterine artery pulsatility index, mean arterial pressure and serum placental growth fac- 
tor at 12, 22 and 32 weeks in prediction of pre-eclampsia. Ultrasound Obstet Gynecol. 2017;50(2):221-7.

33. Khalil A, Maiz N, Garcia-Mandujano R, Penco JM, Nicolaides KH. Longitudinal changes in maternal serum placental growth factor and soluble fms-like tyrosine kinase-1 in women at increased risk of pre-eclampsia. Ultrasound Obstet Gynecol. 2016;47(3):324-31.

34. Rana S, Karumanchi SA, Levine RJ, Venkatesha S, Rauh-Hain JA, Tamez H, et al. Sequential changes in antiangiogenic factors in early pregnancy and risk of developing preeclampsia. Hypertension. 2007;50(1):137-42.

35. Myatt L, Clifton RG, Roberts JM, Spong CY, Wapner RJ, Thorp JM, Jr., et al. Can changes in angiogenic biomarkers between the first and second trimesters of pregnancy predict development of pre-eclampsia in a low-risk nulliparous patient population? BJOG. 2013;120(10):1183-91.

36. Perni U, Sison C, Sharma V, Helseth G, Hawfield A, Suthanthiran M, et al. Angiogenic factors in superimposed preeclampsia: a longitudinal study of women with chronic hypertension during pregnancy. Hypertension. 2012;59(3):740-6.

37. Davis EF, Lazdam M, Lewandowski AJ, Worton SA, Kelly B, Kenworthy Y, et al. Cardiovascular risk factors in children and young adults born to preeclamptic pregnancies: a systematic review. Pediatrics. 2012;129(6):e1552-61.

38. Brown MA, Lindheimer MD, de Swiet M, Van Assche A, Moutquin JM. The classification and diagnosis of the hypertensive disorders of pregnancy: statement from the International Society for the Study of Hypertension in Pregnancy (ISSHP). Hypertens Pregnancy. 2001;20(1):IX-XIV.

39. Rad S, Beauchamp S, Morales C, Mirocha J, Esakoff TF. Defining fetal growth restriction: abdominal circumference as an alternative criterion. J Matern Fetal Neonatal Med. 2018;31(23):3089-94.

40. Powers RW, Jeyabalan A, Clifton RG, Van Dorsten P, Hauth JC, Klebanoff MA, et al. Soluble fms-Like tyrosine kinase 1 (sFlt1), endoglin and placental growth factor (PlGF) in preeclampsia among high risk pregnancies. PLoS One. 2010;5(10):e13263.

41. Ghosh SK, Raheja S, Tuli A, Raghunandan C, Agarwal S. Can maternal serum placental growth factor estimation in early second trimester predict the occurrence of early onset preeclampsia and/or early onset intrauterine growth restriction? A prospective cohort study. J Obstet Gynaecol Res. 2013;39(5):881-90.

42. Tsiakkas A, Cazacu R, Wright A, Wright D, Nicolaides KH. Maternal serum placental growth factor at 12, 22, 32 and 36 weeks' gestation in screening for pre-eclampsia. Ultrasound Obstet Gynecol. 2016;47(4):472-7.

43. MacDonald TM, Tran C, Kaitu'u-Lino TJ, Brennecke SP, Hiscock RJ, Hui L, et al. Assessing the sensitivity of placental growth factor and soluble fms-like tyrosine kinase 1 at 36 weeks' gestation to predict small-for-gestational-age infants or late-onset preeclampsia: a prospective nested case-control study. BMC Pregnancy Childbirth. 2018;18(1):354.

44. Levine RJ, Maynard SE, Qian C, Lim KH, England LJ, Yu KF, et al. Circulating angiogenic factors and the risk of preeclampsia. N Engl J Med. 2004;350(7):672-83.

45. Agrawal S, Cerdeira AS, Redman C, Vatish M. Meta-Analysis and Systematic Review to Assess the Role of Soluble FMS-Like Tyrosine Kinase-1 and Placenta Growth Factor Ratio in Prediction of Preeclampsia: The SaPPPhirE Study. Hypertension. 2018;71(2):306-16. 
46. Dover N, Gulerman HC, Celen S, Kahyaoglu S, Yenicesu O. Placental growth factor: as an early second trimester predictive marker for preeclampsia in normal and high-risk pregnancies in a Turkish population. J Obstet Gynaecol India. 2013;63(3):158-63.

47. Kleinrouweler CE, Wiegerinck MM, Ris-Stalpers C, Bossuyt PM, van der Post JA, von Dadelszen P, et al. Accuracy of circulating placental growth factor, vascular endothelial growth factor, soluble fms-like tyrosine kinase 1 and soluble endoglin in the prediction of pre-eclampsia: a systematic review and meta-analysis. BJOG. 2012;119(7):778-87.

48. Zeisler H, Llurba E, Chantraine F, Vatish M, Staff AC, Sennstrom M, et al. Predictive Value of the sFlt-1:PlGF Ratio in Women with Suspected Preeclampsia. N Engl J Med. 2016;374(1):13-22.

49. Navaratnam K, Abreu P, Clarke H, Jorgensen A, Alfirevic A, Alfirevic Z. Evaluation of agreement of placental growth factor (PIGF) tests and the soluble FMS-like tyrosine kinase 1 (sFlt-1)/PIGF ratio, comparison of predictive accuracy for pre-eclampsia, and relation to uterine artery Doppler and response to aspirin. J Matern Fetal Neonatal Med. 2019;32(2):179-87.

50. Xu B, Shanmugalingam R, Chau K, Pears S, Hennessy A, Makris A. The effect of acetyl salicylic acid (Aspirin) on trophoblast-endothelial interaction in vitro. J Reprod Immunol. 2017;124:54-61.

51. Panagodage S, Yong HE, Da Silva Costa F, Borg AJ, Kalionis B, Brennecke SP, et al. LowDose Acetylsalicylic Acid Treatment Modulates the Production of Cytokines and Improves Trophoblast Function in an in Vitro Model of Early-Onset Preeclampsia. Am J Pathol. 2016;186(12):3217-24.

52. Khalil A, Muttukrishna S, Harrington K, Jauniaux E. Effect of antihypertensive therapy with alpha methyldopa on levels of angiogenic factors in pregnancies with hypertensive disorders. PLoS One. 2008;3(7):e2766.

53. Stepan H, Faber R, Dornhofer N, Huppertz B, Robitzki A, Walther T. New insights into the biology of preeclampsia. Biol Reprod. 2006;74(5):772-6.

54. Xu B, Thornton C, Tooher J, Ogle R, Lim S, Makris A, et al. Effects of anti-hypertensive drugs on production of soluble fms-like tyrosine kinase 1 and soluble endoglin from human normal and pre-eclamptic placentas in vitro. Clin Exp Pharmacol Physiol. 2009;36(8):83942 . 



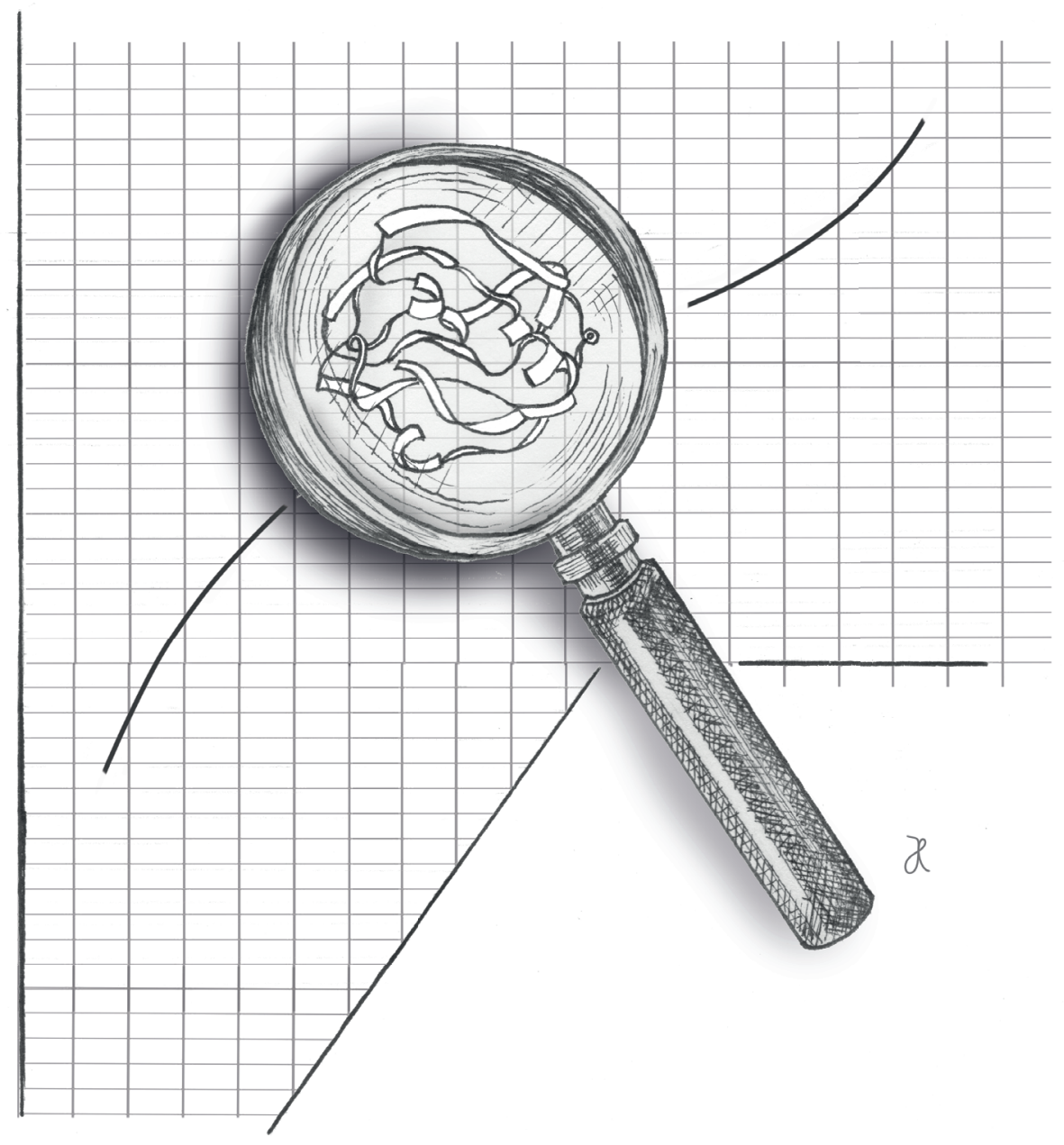


Role of sFlt- 1 and PlGF in the screening of small-for-gestational age neonates during pregnancy: a systematic review

MLE Hendrix

JAP Bons

A van Haren

SMJ van Kuijk

WPTM van Doorn

DM Kimenai

O Bekers

MEA Spaanderman

S Al-Nasiry

Annals of Clinical Biochemistry. 2020 Jan;57(1):44-58 


\section{ABSTRACT}

\section{Background}

Fetal growth restriction (FGR), i.e. the restriction of genetically predetermined growth potential due to placental dysfunction, is a major cause of neonatal morbidity and mortality. The consequences of inadequate fetal growth can be life-long, but the risks can be reduced substantially if the condition is identified prenatally. Currently, screening strategies are based on ultrasound detection of a small-for-gestational age (SGA) fetus, and do not take into account the underlying vascular pathology in the placenta. Measurement of maternal circulating angiogenic biomarkers PlGF (placental growth factor), sFlt-1 (soluble FMS-like tyrosine kinase-1) are increasingly used in studies on FGR as they reflect the pathophysiological process in the placenta. However, interpretation of the role of angiogenic biomarkers in prediction of FGR is hampered by the varying design, population, timing, assay technique and cut-off values used in these studies.

\section{Methods}

We conducted a systematic-review in PubMed (MEDLINE), EMBASE (Ovid) and Cochrane to explore the predictive performance of maternal concentrations of PlGF, sFlt-1 and their ratio for FGR and SGA, at different gestational ages, and describe the longitudinal changes in biomarker concentrations and optimal discriminatory cut-off values.

\section{Results}

We included 26 studies with 2514 cases with SGA, 27 cases of FGR, 582 cases mixed SGA/FGR and 29374 reference. The largest mean differences for the two biomarkers and their ratio were found after 26 weeks of gestational age and not in the first trimester. The ROC AUC varied between 0.60-0.89 with sensitivity and specificity matching the different cut-off values at a preset false positive rate of $10 \%$.

\section{Conclusion}

Most of the studies did not make a distinction between SGA and FGR, and therefore the SGA group consists of fetuses with growth restriction and fetuses that are constitutionally normal. The biomarkers can be a valuable screening tool for SGA pregnancies but unfortunately, there is not yet a clear cut-off value to use for screening. More research is needed to see if these biomarkers are sufficiently able to differentiate growth restriction on their own and how these biomarkers in combination with other relevant clinical and ultrasound parameters can be used in clinical routine diagnostics. 


\section{INTRODUCTION}

It has long been acknowledged that small-for-gestational age (SGA) neonates are at increased risk of perinatal mortality and morbidity and have a higher risk of developing disabilities in childhood, and severe cardiovascular and metabolic diseases later in life $[1,2]$. The obvious reason is that, among the SGA neonates, a subgroup has suffered from intrauterine or fetal growth restriction (FGR), the rest being healthy and constitutionally small due to their demographic factors. Neonates with FGR have failed to reach their genetically predetermined growth potential due to placental dysfunction. The consequences of inadequate fetal growth can be life-long, but the risk of perinatal morbidity and mortality can be reduced substantially if the condition is identified prenatally [1, 3]. An optimal screening strategy for the prediction of SGA and mainly those with fetal growth restriction is highly desirable [4] [5]. Currently, screening strategies focus on antenatal ultrasound to detect small fetuses, defined as a fetal abdominal circumference $(\mathrm{AC})$ or an estimated fetal weight (EFW) below the 10th percentile. However, this ultrasound-based strategy ignores the underlying pathological process in the placenta that leads to suboptimal supply of nutrients to the growing fetus. Abnormal vascular growth and transformation in the placenta can create a high vessel resistance network that has been associated with the development of fetal growth restriction and also preeclampsia [6].

The vascular endothelial growth factor (VEGF) family plays a major role in regulating vasculogenesis and angiogenesis in early human placentation [7]. Placental growth factor (PlGF) is a pro-angiogenic factor predominantly expressed in placental trophoblast and binds to VEGF-receptor or fms-like tyrosine-1 (Flt1), thereby enhancing the pro-angiogenic action of VEGF-A. Besides this membranebound receptor form, Flt-1 also exists in a soluble form (sFlt-1), which is secreted by the placenta and scavenges VEGF and PIGF, thereby lowering circulating levels of unbound VEGF and PlGF. Reduced placental perfusion and subsequent placental hypoxia are associated with increased production of sFlt-1 and decreased concentrations of PIGF in both human and animal models [8]. This altered balance in pro-/anti- angiogenic factors is postulated to cause generalized endothelial dysfunction that is characteristic of preeclampsia which makes endothelial dysfunction a hallmark feature of preeclampsia. Fetal growth restriction has pathophysiological features in common with preeclampsia. Therefore, it was conceivable that measuring these placental biomarkers in maternal blood, could be used in the prediction of preeclampsia and fetal growth restriction [6,9].

The serum sFlt-1/P1GF ratio has already been used in improving the clinical management for preeclampsia. Preeclampsia is defined as the new onset of hyperten- 
sion and proteinuria after 20 weeks of gestation. This disorder is associated with a high risk of fetal growth restriction and maternal morbidity. A low sFlt-1/PlGF ratio was highly predictive for ruling out preeclampsia and avoiding unnecessary hospitalization with a significant economic impact [10]. At this moment there is no conclusive evidence that measuring maternal angiogenic biomarkers is (also) useful in the clinical management of fetal growth restriction. Despite many studies linking maternal angiogenic levels to the neonatal outcomes (mainly SGA), [3, 11-16] there is no systematic review of the predictive performance of PlGF, sFlt-1 and sFlt-1/PIGF ratio at different trimesters of pregnancy.

The main purpose of this systematic review is to assess studies that evaluate the predictive performance of placental angiogenic biomarkers (PlGF, sFlt-1 and/or the sFlt-1/PIGF ratio) for SGA and fetal growth restriction. First, we will describe differences in serum biomarkers levels between SGA and reference pregnancies at different gestational age periods. Next, we will present the longitudinal trajectories of PIGF, sFlt-1 and their ratio for SGA and reference pregnancies. And third, we evaluate the prognostic performance of these biomarkers for the prediction of SGA.

\section{METHODS}

\section{Search Strategy and eligibility criteria}

We performed a systematic search according to the Preferred Reporting Items for Systematic Reviews and Meta-Analyses for Protocols 2015 (PRISMA-P 2015) [17]. The databases PubMed (NCBI), EMBASE (Ovid) and the Cochrane Library were searched for original studies investigating the biomarkers PlGF, sFlt-1 and/ or the sFlt-1/PIGF ratio in SGA or FGR, from database inception until July 27th, 2018. Medical subject headings (MeSH) and text words that we used are listed in Supplementary Table 1, which were combined with Boolean Operators: AND and OR. The included languages were English and Dutch. There was no restriction applied on the type of study. Exclusion criteria were studies where no full text was available, or those performed in multiple pregnancies, nonviable pregnancies, or the measurements being performed after the diagnosis of SGA/FGR was made, as this is in disagreement with the aim of the review. Studies were also excluded if they had no reference group without SGA, did not report absolute values, specific gestational age (GA) timing or the assay methods used of biomarker measurements. Additionally, the reference lists of the selected articles were screened to add all relevant studies. The study protocol was approved by the medical ethical committee of the Maastricht University Medical Centre (17- 
4-0.15.1/ab). All procedures were in accordance with institutional guidelines and adhered to the principles of the Declaration of Helsinki and Title 45, U.S. Code of Federal Regulations, Part 46, Protection of Human Subjects (revised 13 November 2001, effective 13 December 2001).

\section{Study Selection}

An initial title and abstract review was performed by two independent reviewers (M.H. and A.H.), using Rayyan [18]. A third reviewer aided in the exclusion of duplicates and ineligible papers after first selection (S.A.). Next, two investigators analysed the full-text articles to determine their eligibility in the review (M.H. and A.H.) Any disagreement was resolved through discussion. If consensus could not be achieved, a third reviewer (S.A.) was consulted.

\section{Data Extraction}

Data from the eligible studies were extracted by two independent reviewers (M.H. and A.H.) using a predesigned data collection form. The following data were collected: data on first author, publication year, country, type of study design, characteristics of the population studied, sample size, gestational age at sampling, cut-offs used, sensitivity, specificity, area under the curve and assay type. If information was missing, corresponding authors were contacted by email.

\section{Quality Assessment}

The methodological quality of the included studies was assessed using the Quality Assessment of Diagnostic Accuracy Studies-2 (QUADAS-2) tool [19]. The risk of bias is judged in four main domains: 1) patient selection, 2) index test, 3) reference standard and 4) flow of patients in the study and timing of the index test and the reference standard. Concerns about the applicability of the test for clinical practice are also judged in the first three domains.

\section{Data synthesis}

In order to be able to quantitatively asses the relationship between SGA or FGR and the biochemical markers, we converted data given in median or multiple of the median (MoM) with IQR to mean with SD, using an online calculator of the method devised by Hozo et al [20]. Next, the data were categorized by gestational age at time of sampling. The categories made are: 9-14 weeks, 15-25 weeks, 26-35 weeks and $\geq 36$ weeks of gestational age, for both SGA and FGR definitions.

The primary outcome for each study was the mean difference in PlGF, sFlt-1 and their ratio between the SGA and reference group, reported with $95 \%$ confidence interval (CI). 
The ratio between total variation due to heterogeneity and total variation $\left(\mathrm{I}^{2}\right.$ statistics) is presented as a measure for heterogeneity and was used to aid the decision between a fixed effect and random effects meta-analysis. $\mathrm{I}^{2}$ can distinguish true heterogeneity from sampling variance and is expressed as a percentage. Sources of heterogeneity (assay of biomarker measurement, cut-off of SGA) and the difference between reference and SGA pregnancies could not be investigated by meta-regression analyses using a mixed-effects model because of the low amount of studies. Studies were pooled using inverse-variance weighting and were summarized using forest plots. The second outcome was the prognostic performance of these biomarkers and, if available, of the cut-off values to differentiate between SGA versus reference group. The last outcome was the presentation of the longitudinal levels of the PIGF, sFlt- 1 and their ratio for SGA and reference pregnancies, with a difference in assay used. The meta-analyses were performed in $\mathrm{R}$ version 3.2.3 using the meta package.

\section{RESULTS}

\section{Study and data selection}

Our search identified a total of 593 studies, Figure 1. After first selection by title and abstract, duplicates were removed and 489 studies were screened based on title and abstract. Leaving 43 full-text articles to be analysed in detail. In this phase, studies were excluded because of the following reasons: no reference group without SGA $(n=9)$, no absolute values available $(n=5)$, no test method described $(\mathrm{n}=1)$, no full text available $(\mathrm{n}=1)$, no specific defined GA range $(\mathrm{n}=1)$.

The 26 included articles analyzed were published between 2005 and 2018, from all over the world. A total of 2514 cases of SGA, 27 cases of FGR, and 582 cases of mixed SGA/FGR (i.e. both FGR were identified during pregnancy and SGA) were included , in addition to a total of 29374 "reference" pregnancies used as controls in the different studies.

Study characteristics are presented in Table 1 . Most studies employed the case-control design $[2,4,14,21-33]$ and 10 studies were prospective cohorts [1, $3,12,13,15,34-38]$. The majority of studies recruited patients with small-forgestational age as outcome [1-4, 12-15, 21-23, 25, 26, 28-38]. The definition for small-for-gestational age was lower than the $10^{\text {th }}$ birthweight percentiles upper reference limit in 18 studies [3, 4, 12-15, 21-23, 25, 26, 29-32, 34, 35, 37]. Five studies used the cut-off a birthweight lower than the $5^{\text {th }}$ percentile $[1,2,28,36$, 38]. One study used a birthweight lower than the 3rd percentile [24]. One study used a birthweight lower than 2 standard deviations as cut-off value [33]. One 
study used FGR: estimated fetal weight $<10^{\text {th }}$ and an umbilical artery PI $>95^{\text {th }}$ as cut-off value [27]. Three studies described a population with preeclampsia as comorbidity. This differs from 1.3-18.4\% PE in the study population and $2.1-5.0 \%$ in the reference group $[4,24,31]$.

The included studies used a variety of assay platforms from various manufacturers - fifteen used R\&D systems [3, 4, 21, 23-25, 28-33, 35, 37, 38], six studies used Roche Diagnostics [1, 13, 14, 26, 27, 36], two used Thermo Fisher [2, 12], one study Beckman-Coulter [34] , one study Alere [21], one study Bender Medsystems [22] and one study gave no information about the used assay.

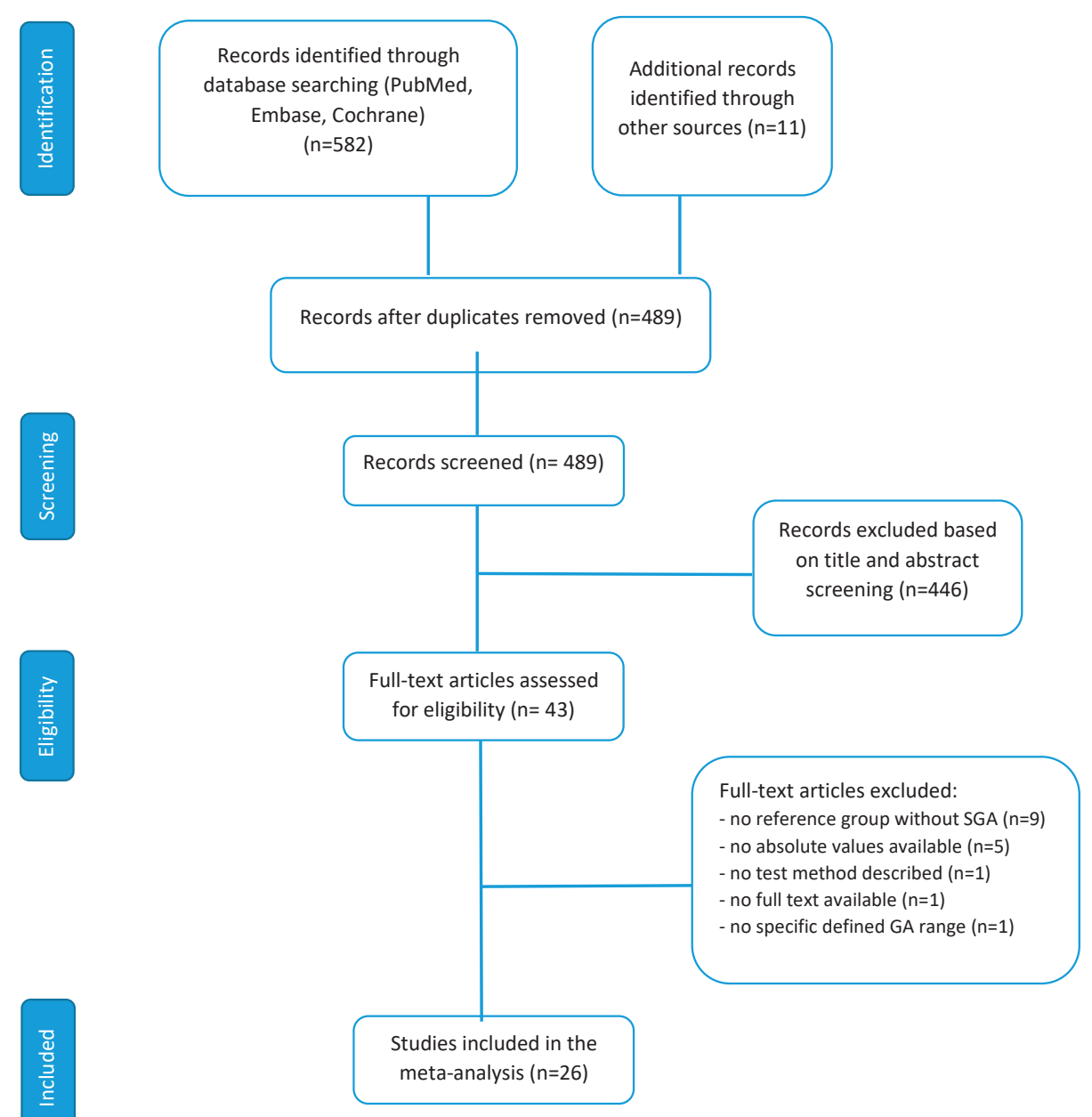

Figure 1 Flowchart summarizing study selection process. 


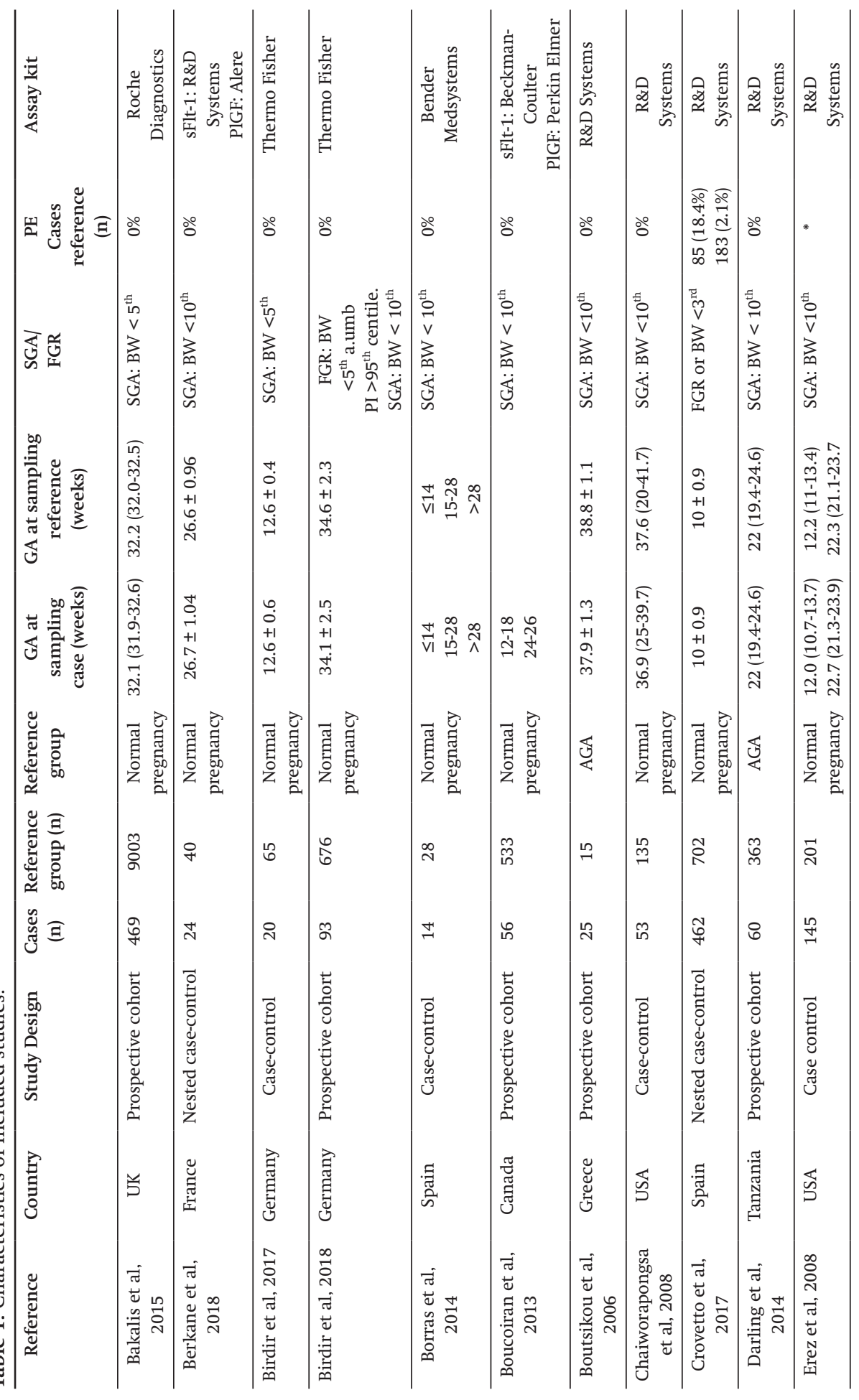




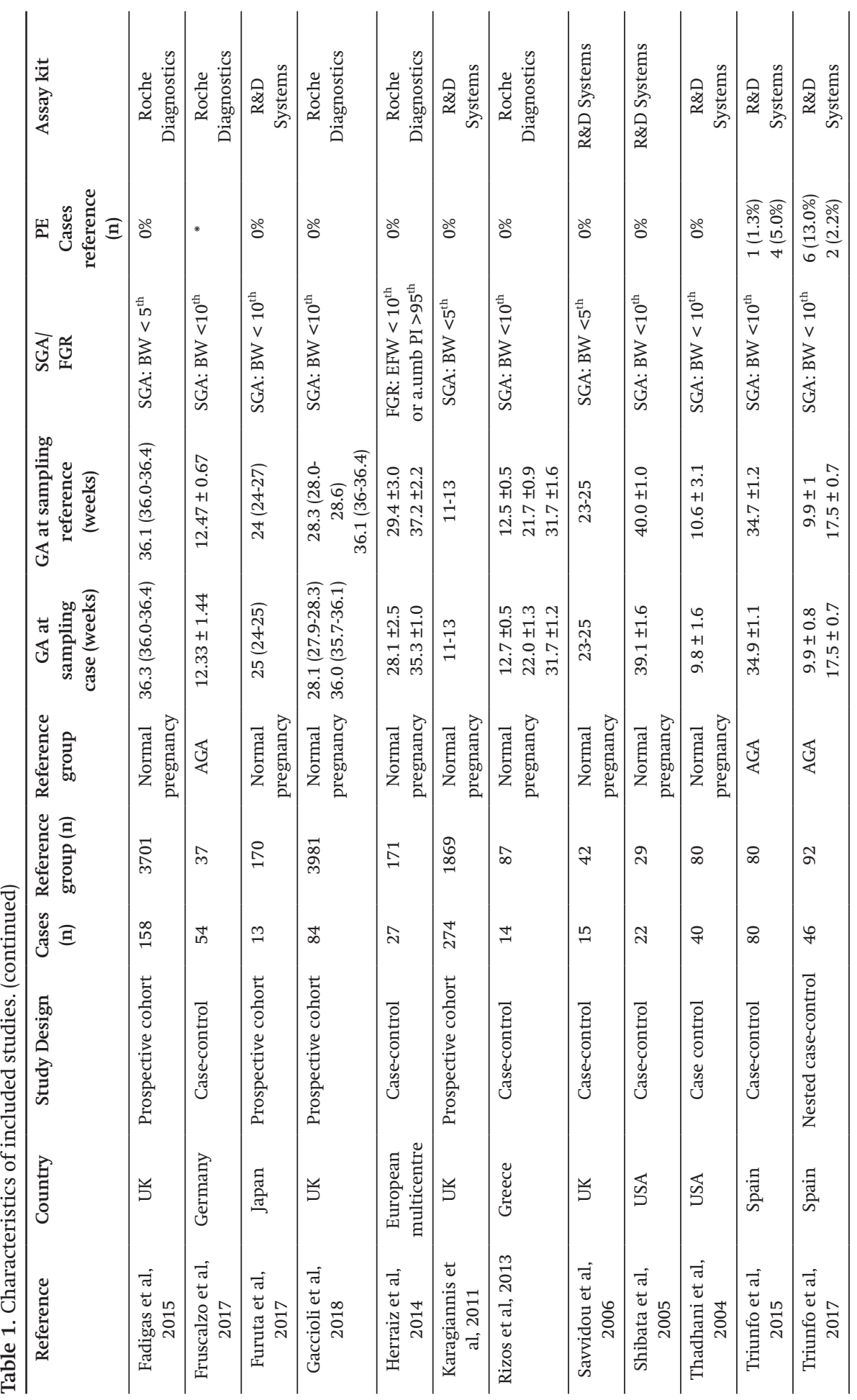




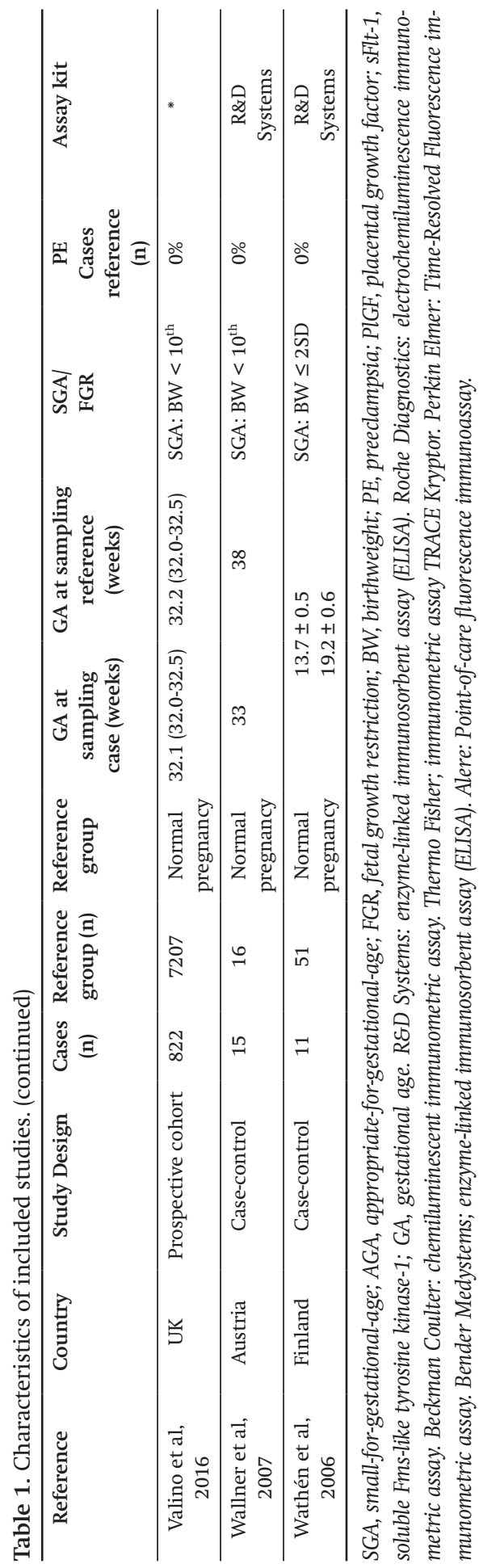




\section{Quality assessment}

The quality assessment of the included studies is depicted in Supplementary Figure 1 . The majority of the studies were defined as good quality $(n=17)$. The other studies were either of moderate quality $(n=6)$ or low quality $(n=3)$. The item that scored lowest among all were patient selection and no details about the used assay.

\section{PIGF measurements during SGA and reference pregnancy}

Forest plots of PIGF measurements during SGA and normal pregnancy are depicted in Figure 2. The PlGF forest plots were divided into 4 gestational age (GA) categories; biomarker measurements $<14$ weeks ( $n=8$ studies), 15-25 weeks ( $n=3$ ), 26-35 weeks $(n=8)$, and $>36$ weeks of GA $(n=3)$. In first and second trimester, we found a minimum change between SGA and reference pregnancies (-5.2 (95\% CI -6.6 to $-3.7 \mathrm{pg} / \mathrm{ml})$ to $-34.5 \mathrm{pg} / \mathrm{ml}$ (95\% CI -69.4 to $0.4 \mathrm{pg} / \mathrm{ml})$. However, after 26

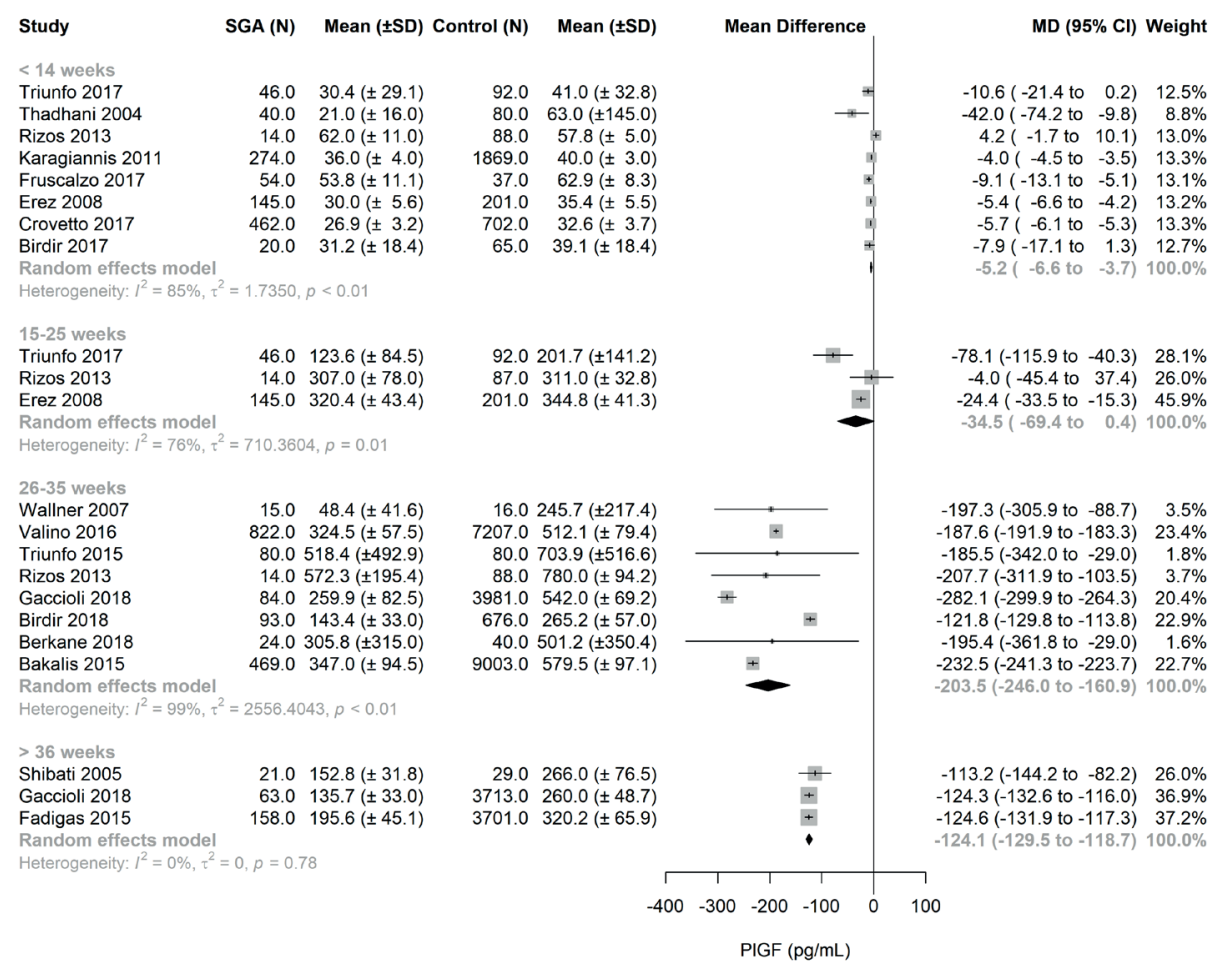

Figure 2 Forest plot of PIGF measurements during SGA and reference pregnancy.

The PIGF forest plot compared SGA with control (reference) group, divided in four GA categories; biomarker measurements < 14 weeks, 15-25 weeks, 26-35 weeks, and > 36 weeks of GA. Only first author of each study is given. GA, gestational age; $M D$, mean difference; $S D$, standard deviation; $C I$, confidence interval. 
weeks forest plots illustrate that SGA group had lower PIGF values compared with reference group (-203.5 pg/ml (95\% CI -246.0 to $-160.9 \mathrm{pg} / \mathrm{ml})$ to -124.1 (95\% CI -129.5 to -118.7$)$ )

\section{sFlt-1 measurements during SGA and reference pregnancy}

There was no significant mean difference during first ( $\mathrm{n}=8$ studies) or second trimester $(n=6)$ between SGA and reference group (Figure 3). After 26 weeks, SGA group had significantly higher sFlt- 1 values compared to the reference group ( $\mathrm{n}=$ 8) with pooled mean difference of $307 \mathrm{pg} / \mathrm{ml}$ (95\% CI 9 to $605 \mathrm{pg} / \mathrm{ml}$ ). The largest mean difference was found after 36 weeks $(n=5)$ with a pooled mean difference of $1152 \mathrm{pg} / \mathrm{ml}$ (95\% CI 317 to $1988 \mathrm{pg} / \mathrm{ml})$.

\begin{tabular}{|c|c|c|}
\hline Study & SGA (N) & Mean ( $\pm S D)$ \\
\hline \multicolumn{3}{|l|}{$<14$ weeks } \\
\hline Birdir 2017 & 20 & $1252( \pm 704)$ \\
\hline Crovetto 2017 & 462 & $1508( \pm 133)$ \\
\hline Erez 2008 & 145 & $1616( \pm 165)$ \\
\hline Fruscalzo 2017 & 54 & $1197( \pm 290)$ \\
\hline Rizos 2013 & 14 & $1208( \pm 256)$ \\
\hline Thadhani 2004 & 40 & $1101( \pm 479)$ \\
\hline Triunfo 2017 & 46 & $1805( \pm 682)$ \\
\hline Wathen 2006 & 11 & $462( \pm 83)$ \\
\hline \multirow{2}{*}{\multicolumn{3}{|c|}{$\begin{array}{l}\text { Random effects model } \\
\text { Heterogeneity: } I^{2}=98 \%, \tau^{2}=35301.6537, p<0.01\end{array}$}} \\
\hline & & \\
\hline \multicolumn{3}{|l|}{$15-25$ weeks } \\
\hline Darling 2014 & 60 & $940( \pm 538)$ \\
\hline Erez 2008 & 145 & $1687( \pm 219)$ \\
\hline Rizos 2013 & 14 & $1068( \pm 321)$ \\
\hline Savvidou 2006 & 15 & $1725( \pm 378)$ \\
\hline Triunfo 2017 & 46 & $1566( \pm 815)$ \\
\hline Wathen 2006 & 10 & $362( \pm 65)$ \\
\hline \multirow{2}{*}{\multicolumn{3}{|c|}{$\begin{array}{l}\text { Random effects model } \\
\text { Heterogeneity: } I^{2}=98 \%, \tau^{2}=99546.8066, p<0.01\end{array}$}} \\
\hline & & \\
\hline \multicolumn{3}{|l|}{ 26-35 weeks } \\
\hline Berkane 2018 & & $2505( \pm 1784)$ \\
\hline Birdir 2018 & 93 & $2657( \pm 729)$ \\
\hline Bakalis 2015 & 371 & $2042( \pm 182)$ \\
\hline Gaccioli 2018 & 84 & $2114( \pm 496)$ \\
\hline Rizos 2013 & 14 & $1324( \pm 372)$ \\
\hline Triunfo 2015 & 80 & $3021( \pm 1791)$ \\
\hline Valino 2016 & 822 & $1568( \pm 212)$ \\
\hline Wallner 2007 & 15 & $4479( \pm 2633)$ \\
\hline \multicolumn{3}{|c|}{ Random effects model } \\
\hline \multicolumn{3}{|c|}{ Heterogeneity: $I^{2}=100 \%, \tau^{2}=141556.6756, p=0$} \\
\hline \multicolumn{3}{|l|}{$>36$ weeks } \\
\hline Boutsikou 2006 & 25 & $3416( \pm 1528)$ \\
\hline Chaiworapongsa 2008 & 53 & $3603( \pm 6740)$ \\
\hline Fadigas 2015 & 158 & $2909( \pm 408)$ \\
\hline Gaccioli 2018 & 63 & $5333( \pm 1424)$ \\
\hline Shibata 2015 & 22 & $2227( \pm 514)$ \\
\hline \multicolumn{3}{|l|}{ Random effects model } \\
\hline Heterogeneity: $I^{2}=97 \%$ & & 9.5955 , \\
\hline
\end{tabular}

\section{ol (N) Mean (士SD)}

$651216( \pm 523)$

$7021397( \pm 168)$

$2011788( \pm 178)$

$371610( \pm 256)$

$88 \quad 1530( \pm 122)$

$80 \quad 973( \pm 490)$

$921887( \pm 702)$

$51 \quad 432( \pm 85)$

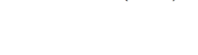

$3631310( \pm 593)$

$2011800( \pm 246)$

$87 \quad 1428( \pm 180)$

$42 \quad 463( \pm 113)$

$921474( \pm 612)$

$36 \quad 296( \pm 81)$
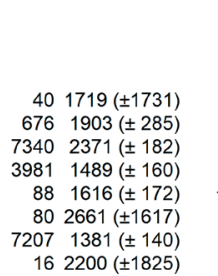

$162200( \pm 1825)$

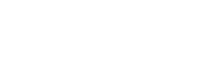

$15 \quad 1722( \pm 533)$

$\begin{array}{rr}135 & 1445( \pm 865) \\ 3701 & 2460( \pm 269)\end{array}$

$37133013( \pm 343)$

$292472( \pm 318)$

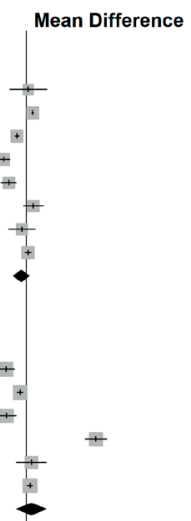

MD $(95 \% \mathrm{Cl})$ Weight

36 (-298 to 369$) \quad 9.8 \%$

111 ( 94 to 128 ) $13.7 \%$

$-172(-209$ to -136$) \quad 13.6 \%$

$-413(-526$ to -300$) \quad 13.1 \%$

$-322(-458$ to -185$) \quad 12.8 \%$

128 ( -55 to 311$) \quad 12.2 \%$

$-82(-326$ to 162$) \quad 11.3 \%$

30 (-25 to 84$) \quad 13.5 \%$

$-92(-233$ to 49$) 100.0 \%$

-370 (-519 to -221$) \quad 16.9 \%$ $-112(-162$ to -63$) \quad 18.0 \%$ -360 (-533 to -188$) \quad 16.5 \%$ 1262 (1068 to 1456 ) $16.1 \%$ 91 (-175 to 358$) \quad 14.5 \%$ 66 ( 17 to 114$) \quad 18.0 \%$ 88 (-173 to 349$) 100.0 \%$

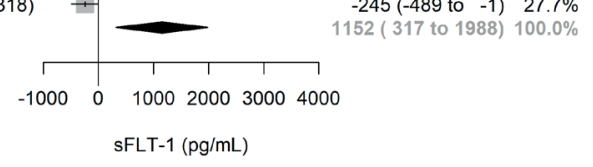

Figure 3 Forest plot of sFlt- 1 measurements during SGA and reference pregnancy.

The sFit-1 forest plot compared SGA with control (reference) group, divided in four GA categories; biomarker measurements <14 weeks, 15-25 weeks, 26-35 weeks, and >36 weeks of GA. Only first author of each study is given. GA, gestational age; $\mathrm{MD}$, mean difference; SD, standard deviation; $C I$, confidence interval. 


\section{sFlt-1/PIGF ratio during SGA and reference pregnancy}

The sFlt-1/PIGF measurement during SGA pregnancies compared with reference values are depicted in Figure 4. No significant mean difference was found between SGA and reference for the sFlt-1/P1GF ratio during different gestational age intervals: <14 weeks, 15-25 weeks and 26-36 weeks $(n=5,2$ and 5 studies, respectively). The sFlt-1/P1GF ratio values were higher in the SGA group versus reference only after 36 weeks based on one study with a mean difference of 30.1 (95\% CI $24.8-35.4)$.

PlGF longitudinal courses show the most significant differences between SGA and control pregnancies, whereas the differences in the sFLT-1 and ratio courses between the two groups are minimal.

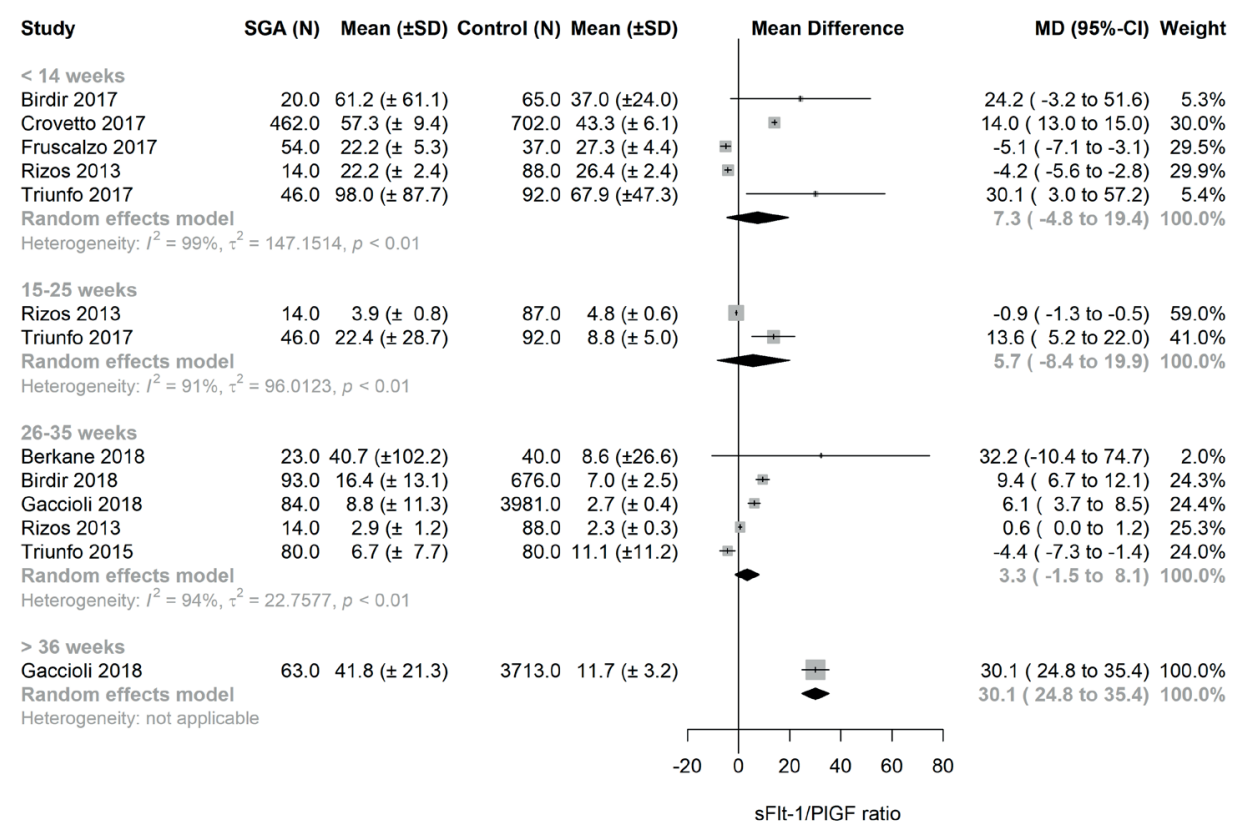

Figure 4 Forest plot of sFlt-1/PIGF measurements during SGA and reference pregnancy.

The sFlt-1/PlGF forest plot compared SGA with control (reference) group, divided in four GA categories; biomarker measurements <14 weeks, 15-25 weeks, 26-35 weeks, and >36 weeks of GA. Only first author of each study is given. GA, gestational age; $M D$, mean difference; SD, standard deviation; CI, confidence interval.

\section{Longitudinal course of PIGF, sFlt-1 and ratio during SGA pregnancy and reference}

In Figure 5, 6 and 7 the longitudinal course of PlGF, sFlt-1 and the ratio are presented for both the SGA and the reference group. In reference pregnancies, PIGF levels were low in the first trimester, followed by a steady increase starting from 12 weeks till a peak around 30 weeks, followed by a sharp drop until term. SGA 
pregnancies had a similar but blunter curve with consistently lower PIGF levels, mainly between 24-36 weeks. Levels of sFlt-1 in reference pregnancies showed a dual peak, around 16 weeks and again after 30 weeks until term. SGA pregnancies had a grossly similar curve with demonstrably higher sFlt-1 levels after 30 weeks until term. The sFlt-1/PlGF ratio in reference pregnancies showed a steady decrease throughout pregnancy with a nadir around 30 weeks, and a slight shallow increase thereafter until term. Similar to the sFlt-1 curve, SGA pregnancies had grossly a similar curve for the sFlt-1/PlGF ratio with an earlier and steeper rise from 28 weeks onwards.

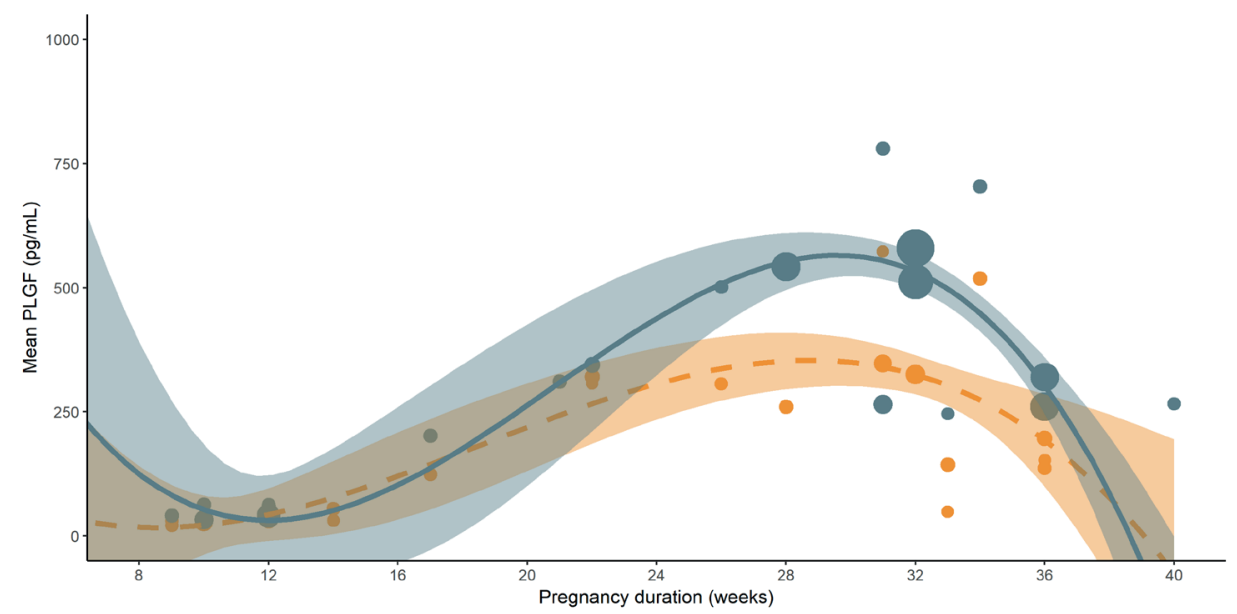

Figure 5 Longitudinal course of PlGF.

SGA pregnancies in orange. Reference pregnancies in blue. The dots present the individual studies. The number of cases and controls included in the study influence the size of dots. Pregnancy duration in weeks.

\section{Prognostic performance of the biomarkers}

In Table 2, studies reporting sensitivity, specificity, positive and negative predictive values, and area under the receiver operating characteristic curve (AUC) for predicting FGR or SGA using PIGF (8 studies), sFlt-1 (4 studies), and sFlt-1/PlGF ratio (8 studies) were included. Pooled analysis of prediction parameters was not possible due to the different gestational age at sampling and different definitions used for SGA (birthweight $<5^{\text {th }}$ or $<10^{\text {th }}$ percentile) and FGR.

For PlGF only 4 of the 8 studies reported cut-off values using different assays and for the two studies which used the Thermo Fisher assay also different cut-off values were used. The AUC values varied from 0.65 to 0.89 , reflecting the wide range in cut-off values used: 34.7 (at 12 weeks) [12] to 252 (at 28 weeks) [13]. For sFlt- 1 two of the four studies reported cut-off values and these 2 studies used different assay types. The AUC values vary from 0.61 to 0.74 . For the sFlt-1/PlGF 


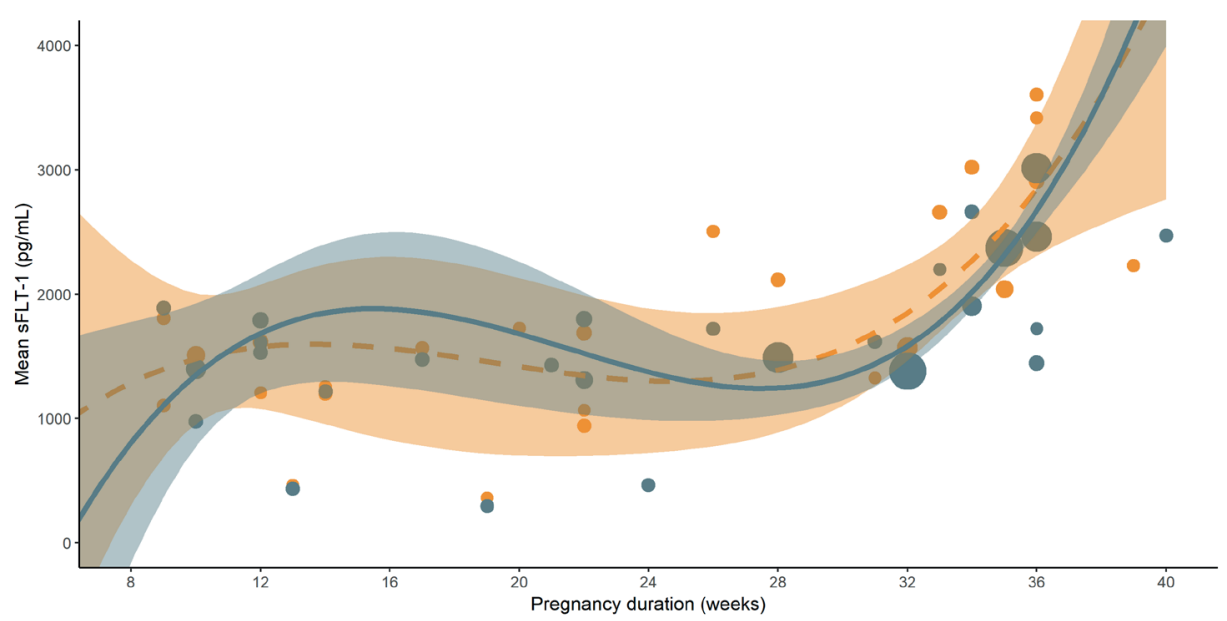

Figure 6 Longitudinal course of sFlt-1

SGA pregnancies in orange. Reference pregnancies in blue. The dots present the individual studies. The number of cases and controls included in the study influence the size of the dots. Pregnancy duration in weeks.

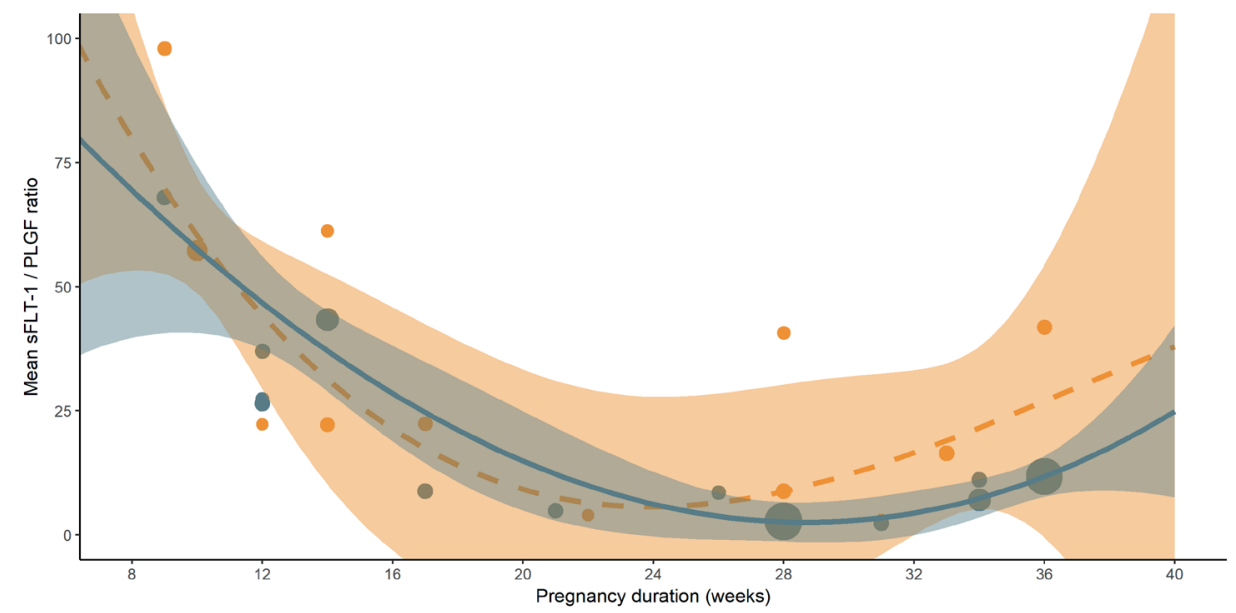

Figure 7 Longitudinal course of sFlt-1/PIGF ratio.

SGA pregnancies in orange. Reference pregnancies in blue. The dots present the individual studies. The number of cases and controls included in the study influence the size of the dots. Pregnancy duration in weeks.

ratio, 5 out of 8 studies reported cut-off values and sometimes more than one cut-off value was used [13, 27]. The AUC values vary from 0.55 to 0.89 .

Five studies used a combination of maternal, fetal and biochemical factors in the prediction of SGA. In the first trimester, a model using serum PIGF levels, in combination with maternal factors, mean arterial pressure (MAP), pregnancyassociated plasma protein-A (PAPP-A) and beta-human chorionic gonadotropin $(\beta$-hCG) had a prediction rate of $73 \%$ for early SGA (requiring delivery before 37 


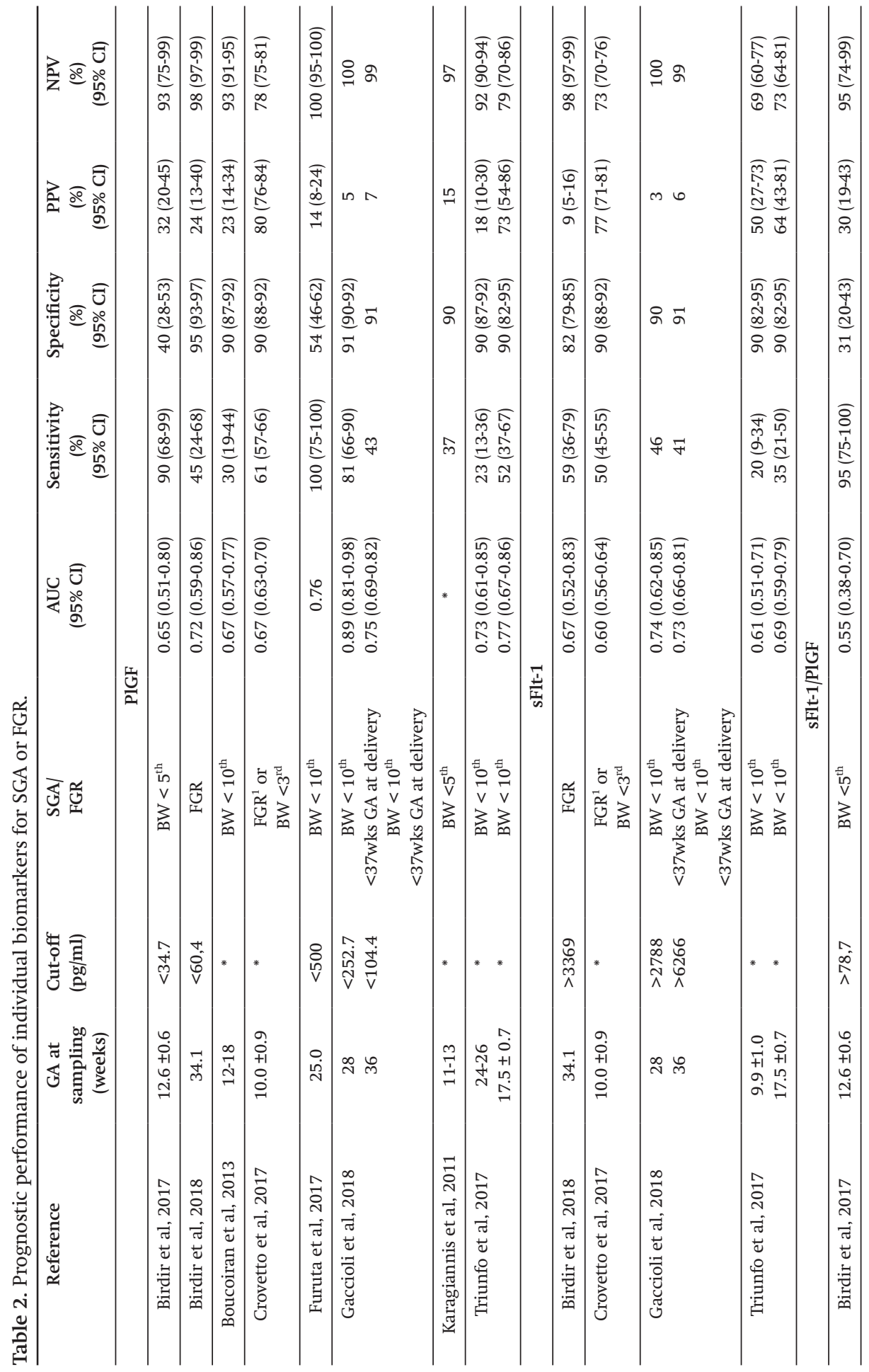




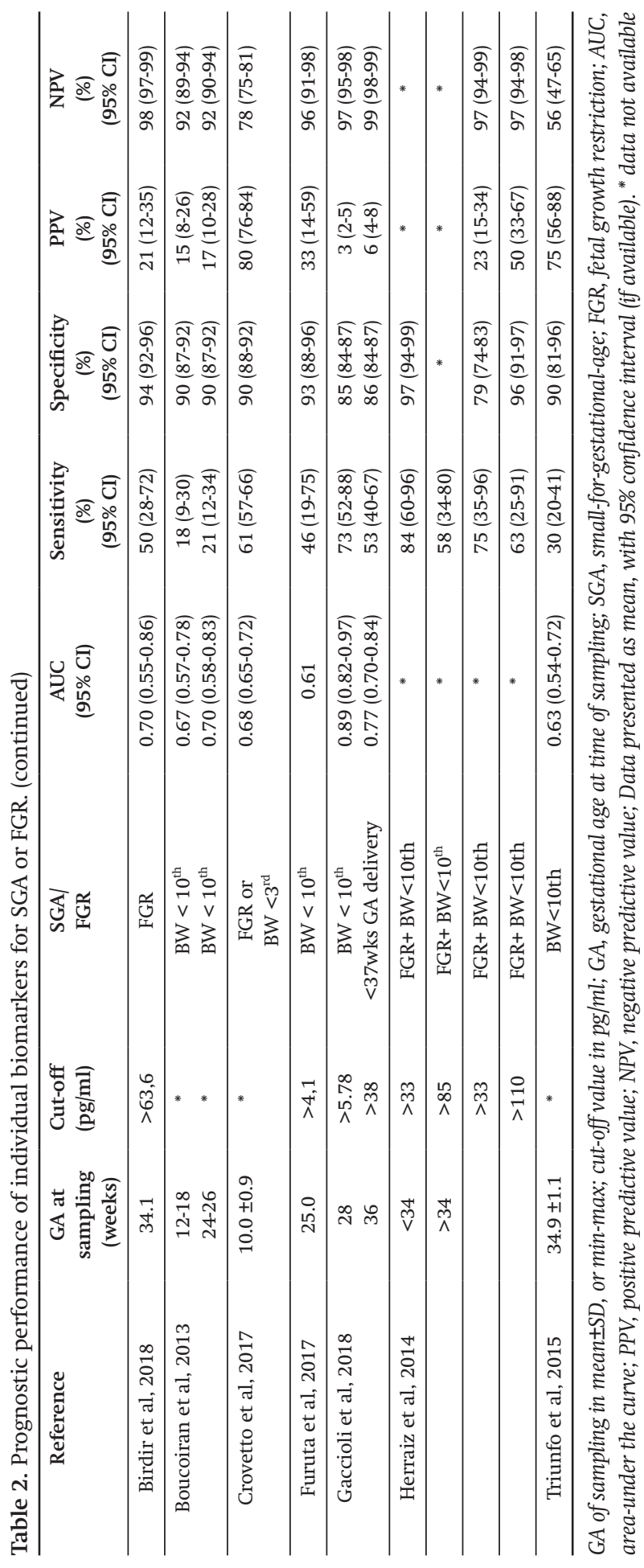


weeks) and 46\% for late SGA (delivering at term) with a FPR of 10\% [38]. A model using a combination of sFlt-1/PlGF ratio and a-priori risk for SGA, MAP and uterine artery pulsatility index (UtA-PI) had a prediction rate of $42 \%$ for SGA and $66 \%$ for FGR, with a FPR of $10 \%$ [24].

In third trimester, serum PlGF levels were used in two models in combination with maternal factors and EFW to predict SGA with a higher sensitivity for early SGA (delivering at 32-36 weeks) compared to late SGA (delivering >37 weeks) and for severe SGA (birthweight $<3^{\text {rd }}$ percentile) compared to $\left(<10^{\text {th }}\right)[1,36]$. Using a combination of sFlt-1/PIGF ratio and EFW the prediction rate for SGA was $45 \%$, with a 10\% FPR and AUC of 0.77 (95\% CI 0.70-0.85) [31].

\section{DISCUSSION}

In this systematic review, we included 26 studies that investigated the values of measuring the angiogenic biomarkers PlGF, sFlt-1 and their ratio in pregnancies complicated by FGR or SGA. This is the first review to our knowledge, that focused on studies describing biomarkers changes in SGA pregnancies. Using gestational age-specific intervals, the data analysis demonstrated that the mean differences between SGA and reference pregnancies were larger in serum level of PIGF than with sFlt-1 or their ratio, and were more profound during later stages of pregnancy (after 25 weeks) and not in the first trimester.

In the longitudinal course of biomarkers there was a good discrimination between SGA and reference pregnancies especially during 24-36 weeks for PIGF, while for sFlt-1 and the sFlt-1/PlGF ratio there was no discrimination between SGA and reference pregnancies as depicted by the overlapping confidence intervals. The amount of literature for sFlt- 1 and the sFlt-1/PlGF ratio is limited and also heterogenous which can result in a diminished discrimination between SGA and reference pregnancies for sFlt-1 and the sFlt-1/PlGF ratio. Furthermore, screening with biomarkers for SGA showed a moderate predictive performance with a wide range of AUC that varied from $0.65-0.89$ for PlGF, 0.55-0.89 for sFlt- 1 and the sFlt-1/ PlGF ratio, reflecting the heterogeneity in outcome definition, assay methods and timing of screening.

Angiogenic biomarkers, as noninvasive and readily available indicators of placental function have made a large impact on the way researchers nowadays understand and approach placental dysfunction disorders [39]. Since the earlier publications describing lower maternal serum PIGF and higher sFlt-1 levels in women with preeclampsia [40], these biomarkers have increasingly claimed a prominent place in the clinical management of preeclampsia. Using a cut-off 
value of 38 , the sFlt-1/P1GF ratio as proposed is a reliable predictor for the imminent development of preeclampsia [10,41].

Since preeclampsia and FGR probably share a common underlying placental pathology [42], we aimed in this review that identifying cut-off values for these biomarkers could be of use to differentiate between pregnancies with or without FGR. Only 3 out of 26 studies had patients included with preeclampsia.

Because of this relative low amount of studies, we could not perform a reliable analysis with PE. An optimal discriminatory cut-off value could not be calculated from published studies, neither for the outcome of FGR nor for SGA due to the limited number of studies reporting cut-off values used (4 out of 8 studies) and their heterogeneous setting. In addition, pooled analysis of prediction parameters was also not possible due to the different assay methods, different gestational age at sampling and different definitions used for SGA (birthweight $<5^{\text {th }}$ or $<10^{\text {th }}$ percentile) and FGR. Benton et al [11] showed that low PlGF levels $\left(<5^{\text {th }}\right.$ percentile for gestational age) had a good capacity (AUC of 0.96) to discriminate fetuses with placental disease from those who are constitutionally-small. Using gestational age-adjusted percentiles for PlGF could, therefore, improve clinical management of pregnancies with SGA by identifying the subgroup with an underlying placental pathology and ultimately form the highest risk group.

We and others have demonstrated the added value of combining the measurement of placental biomarkers in the first trimester with clinical characteristics, Doppler parameters or fetal growth in the prediction of FGR [38, 43, 44]. The use of multiple parameters could increase the specificity and sensitivity of the screening, mainly because each biomarker reflects a different mechanistic pathway linked to the disease process. While abnormal uterine artery Doppler reflects the inadequate trophoblastic invasion of the maternal spiral arteries, abnormal serum levels of PIGF and sFlt-1 reflect the dysregulated secretory activity by placental trophoblasts [6], both well recognized mechanisms linked to the development of FGR. Earlier screening, before evident clinical recognition is possible, may have the potential to improve the clinical management and reduced morbidity for both mother and fetus. Consequently, appropriate planning of follow-up visits and the timely commencement of therapeutic interventions, such as Aspirin is possible, with recent large trials reporting great success in reversing the natural outcome of placenta dysfunction [45].

This review highlights the importance of meticulous consideration of methodological aspects of studies, that might affect the interpretation of results. The considerable heterogeneity between the studies included, as reflected by high $\mathrm{I}^{2}$ values in the forest plot analysis, could have various sources. Worldwide, different fetal and neonatal growth charts are used for defining FGR and SGA with 
varying cut-off values. It is difficult to define FGR, the fetus does not reach its biological growth potential as a consequence of impaired placental function. It is usually defined by the statistical deviation of fetal size from a population-based reference, with a threshold at the $10^{\text {th }}, 5^{\text {th }}$ of $3^{\text {rd }}$ centile. However this threshold is better usable for SGA, based on actual weight at birth. Due to the lack of this gold standard to define FGR based on the underlying pathophysiology, SGA is used as an inappropriate proxy, that represents a heterogeneous group of both constitutionally small and growth restricted fetuses [46]. Growth-restricted fetuses with a birth weight $>10^{\text {th }}$ centile, may not reach their growth potential, but remain undiagnosed because of the birthweight, with an increased risk of adverse outcome. Recently, consensus definitions have been reached by Delphi procedure for both early-onset and late-onset FGR [47]. These new definitions of FGR are useful for a prospective study in the future, many of the biomarker studies in this review have been published before the new definition.

The heterogeneity could also be caused by varying study design and using different assays. The studies included in this review used different immunoassays for PIGF and sFlt-1. It is well-known that standardization of immunoassays is difficult. A major problem in immunoassays is that the recognition of the analyte is determined by the reagent using a limited portion of the molecule [48]. Large differences in assay calibration can lead to misinterpretations of the clinical course when different assays are used [49]. For PIGF and sFlt-1 there is no reference method defined and there is no reference material, like a WHO standard available. The different antibodies which are used can have another specificity for the PIGF isoforms and to free and bound PIGF. In addition, the larger studies which included more than 100 SGA cases or controls were also based on R\&D Systems and Roche assays. Although, there is no reference method or material available for PIGF and sFlt-1, it is described that the PlGF and sFlt- 1 immunoassays of Roche Diagnostics are calibrated with recombinant human PIGF and sFlt-1 and standardized against the Quantikine PIGF and sFlt-1 ELISAs from R\&D Systems [50]. The study of Schiettecatte et al 2010 showed that there is good correlation between Roche and the Quantikine ELISA R\&D Systems [51]. The Kryptor/Thermo Fisher PlGF method is also calibrated with recombinant human PlGF and standardized against the Quantikine PIGF ELISA from R\&D system. The Kryptor sFlt-1 method is standardized against the Roche method [52]. The study of Helden et al 2015 confirmed a good correlation between Kryptor and Roche assay [53]. So we can conclude that the results of the R\&D system, Roche and Thermo Fisher assays are very similar because they are standardized against each other [54]. 


\section{CONCLUSION}

In conclusion, this review outlines the differences in angiogenic biomarker levels in SGA/FGR pregnancies, similar to earlier reviews on the related placental disorder of preeclampsia. Despite variation in study design and test methods, angiogenic biomarkers showed different mean levels and longitudinal trends in SGA pregnancies. Further research should focus on improving the prediction of at-risk SGA pregnancies by combining placental biomarkers with clinical characteristics and ultrasound measures of fetal growth velocity. 


\section{REFERENCES}

1. Bakalis, S., et al., Prediction of small-for-gestational-age neonates: screening by biophysical and biochemical markers at 30-34 weeks. Ultrasound Obstet Gynecol, 2015. 46(4): p. 446-51.

2. Birdir, C., et al., Impact of maternal serum levels of Visfatin, AFP, PAPP-A, sFlt-1 and PIGF at 11-13 weeks gestation on small for gestational age births. J Matern Fetal Neonatal Med, 2017. 30(6): p. 629-634.

3. Darling, A.M., et al., Angiogenic and inflammatory biomarkers in midpregnancy and small-for-gestational-age outcomes in Tanzania. Am J Obstet Gynecol, 2014. 211(5): p. 509 e1-8.

4. Triunfo, S., et al., Changes in uterine artery Doppler velocimetry and circulating angiogenic factors in the first half of pregnancies delivering a small-for-gestational-age neonate. Ultrasound Obstet Gynecol, 2017. 49(3): p. 357-363.

5. Lindqvist, P.G. and J. Molin, Does antenatal identification of small-for-gestational age fetuses significantly improve their outcome? Ultrasound Obstet Gynecol, 2005. 25(3): p. 258-64.

6. Vrachnis, N., et al., Placental growth factor (PlGF): a key to optimizing fetal growth. J Matern Fetal Neonatal Med, 2013. 26(10): p. 995-1002.

7. Shibuya, M., Vascular Endothelial Growth Factor (VEGF) and Its Receptor (VEGFR) Signaling in Angiogenesis: A Crucial Target for Anti- and Pro-Angiogenic Therapies. Genes Cancer, 2011. 2(12): p. 1097-105.

8. Chau, K., A. Hennessy, and A. Makris, Placental growth factor and pre-eclampsia. J Hum Hypertens, 2017. 31(12): p. 782-786.

9. MacDonald, T.M., et al., Assessing the sensitivity of placental growth factor and soluble fms-like tyrosine kinase 1 at 36 weeks' gestation to predict small-for-gestational-age infants or late-onset preeclampsia: a prospective nested case-control study. BMC Pregnancy Childbirth, 2018. 18(1): p. 354.

10. Caillon, H., et al., Evaluation of sFlt-1/PlGF Ratio for Predicting and Improving Clinical Management of Pre-eclampsia: Experience in a Specialized Perinatal Care Center. Ann Lab Med, 2018. 38(2): p. 95-101.

11. Benton, S.J., et al., Placental growth factor as a marker of fetal growth restriction caused by placental dysfunction. Placenta, 2016. 42: p. 1-8.

12. Birdir, C., et al., Predictive value of sFlt-1, PIGF, sFlt-1/PlGF ratio and PAPP-A for late-onset preeclampsia and IUGR between 32 and 37weeks of pregnancy. Pregnancy Hypertens, 2018. 12: p. 124-128.

13. Gaccioli, F., et al., Screening for fetal growth restriction using ultrasound and the sFLT1/ PlGF ratio in nulliparous women: a prospective cohort study. Lancet Child Adolesc Health, 2018. 2(8): p. 569-581.

14. Rizos, D., et al., Placental growth factor and soluble fms-like tyrosine kinase-1 are useful markers for the prediction of preeclampsia but not for small for gestational age neonates: a longitudinal study. Eur J Obstet Gynecol Reprod Biol, 2013. 171(2): p. 225-30.

15. Valino, N., et al., Biophysical and biochemical markers at 30-34 weeks' gestation in the prediction of adverse perinatal outcome. Ultrasound Obstet Gynecol, 2016. 47(2): p. 194202. 
16. Asvold, B.O., et al., Angiogenic factors in maternal circulation and the risk of severe fetal growth restriction. Am J Epidemiol, 2011. 173(6): p. 630-9.

17. Moher, D., et al., Preferred reporting items for systematic review and meta-analysis protocols (PRISMA-P) 2015 statement. Syst Rev, 2015. 4: p. 1.

18. Ouzzani, M., et al., Rayyan-a web and mobile app for systematic reviews. Syst Rev, 2016. 5(1): p. 210.

19. Whiting, P.F., et al., QUADAS-2: a revised tool for the quality assessment of diagnostic accuracy studies. Ann Intern Med, 2011. 155(8): p. 529-36.

20. Hozo, S.P., B. Djulbegovic, and I. Hozo, Estimating the mean and variance from the median, range, and the size of a sample. BMC Med Res Methodol, 2005. 5: p. 13.

21. Berkane, N., et al., Abnormal steroidogenesis and aromatase activity in preeclampsia. Placenta, 2018. 69: p. 40-49.

22. Borras, D., et al., Angiogenic growth factors in maternal and fetal serum in pregnancies complicated with intrauterine growth restriction. J Obstet Gynaecol, 2014. 34(3): p. 21820.

23. Chaiworapongsa, T., et al., The maternal plasma soluble vascular endothelial growth factor receptor-1 concentration is elevated in SGA and the magnitude of the increase relates to Doppler abnormalities in the maternal and fetal circulation. J Matern Fetal Neonatal Med, 2008. 21(1): p. 25-40.

24. Crovetto, F., et al., Differential performance of first-trimester screening in predicting small-for-gestational-age neonate or fetal growth restriction. Ultrasound Obstet Gynecol, 2017. 49(3): p. 349-356.

25. Erez, O., et al., The change in concentrations of angiogenic and anti-angiogenic factors in maternal plasma between the first and second trimesters in risk assessment for the subsequent development of preeclampsia and small-for-gestational age. J Matern Fetal Neonatal Med, 2008. 21(5): p. 279-87.

26. Fruscalzo, A., et al., First trimester TTR-RBP4-ROH complex and angiogenic factors in the prediction of small for gestational age infant's outcome. Arch Gynecol Obstet, 2017. 295(5): p. 1157-1165.

27. Herraiz, I., et al., Characterization of the soluble fms-like tyrosine kinase-1 to placental growth factor ratio in pregnancies complicated by fetal growth restriction. Obstet Gynecol, 2014. 124(2 Pt 1): p. 265-73.

28. Savvidou, M.D., et al., Maternal serum concentration of soluble fms-like tyrosine kinase 1 and vascular endothelial growth factor in women with abnormal uterine artery Doppler and in those with fetal growth restriction. Am J Obstet Gynecol, 2006. 195(6): p. 1668-73.

29. Shibata, E., et al., Soluble fms-like tyrosine kinase 1 is increased in preeclampsia but not in normotensive pregnancies with small-for-gestational-age neonates: relationship to circulating placental growth factor. J Clin Endocrinol Metab, 2005. 90(8): p. 4895-903.

30. Thadhani, R., et al., First Trimester Placental Growth Factor and Soluble Fms-Like Tyrosine Kinase 1 and Risk for Preeclampsia. Journal of Clinical Endocrinology and Metabolism, 2004. 89(2): p. 770-775.

31. Triunfo, S., et al., Angiogenic Factors and Doppler Evaluation in Normally Growing Fetuses at Routine Third-Trimester Scan: Prediction of Subsequent Low Birth Weight. Fetal Diagn Ther, 2016. 40(1): p. 13-20.

32. Wallner, W., et al., Angiogenic growth factors in maternal and fetal serum in pregnancies complicated by intrauterine growth restriction. Clin Sci (Lond), 2007. 112(1): p. 51-7. 
33. Wathen, K.A., et al., Maternal serum-soluble vascular endothelial growth factor receptor-1 in early pregnancy ending in preeclampsia or intrauterine growth retardation. J Clin Endocrinol Metab, 2006. 91(1): p. 180-4.

34. Boucoiran, I., et al., Risks for preeclampsia and small for gestational age: predictive values of placental growth factor, soluble fms-like tyrosine kinase-1, and inhibin A in singleton and multiple-gestation pregnancies. Am J Perinatol, 2013. 30(7): p. 607-12.

35. Boutsikou, T., et al., Soluble vascular endothelial growth factor receptor-1 in intrauterine growth restricted fetuses and neonates. Early Hum Dev, 2006. 82(4): p. 235-9.

36. Fadigas, C., et al., Prediction of small-for-gestational-age neonates: screening by placental growth factor and soluble fms-like tyrosine kinase-1 at 35-37 weeks. Ultrasound Obstet Gynecol, 2015. 46(2): p. 191-7.

37. Furuta, I., et al., Serum placental growth factor and soluble fms-like tyrosine kinase 1 at mid-gestation in healthy women: Association with small-for-gestational-age neonates. J Obstet Gynaecol Res, 2017. 43(7): p. 1152-1158.

38. Karagiannis, G., et al., Prediction of small-for-gestation neonates from biophysical and biochemical markers at 11-13 weeks. Fetal Diagn Ther, 2011. 29(2): p. 148-54.

39. Stepan, H., et al., Implementation of the sFlt-1/PIGF ratio for prediction and diagnosis of pre-eclampsia in singleton pregnancy: implications for clinical practice. Ultrasound Obstet Gynecol, 2015. 45(3): p. 241-6.

40. Maynard, S.E., et al., Excess placental soluble fms-like tyrosine kinase 1 (sFlt1) may contribute to endothelial dysfunction, hypertension, and proteinuria in preeclampsia. J Clin Invest, 2003. 111(5): p. 649-58.

41. Zeisler, H., et al., Predictive Value of the sFlt-1:PlGF Ratio in Women with Suspected Preeclampsia. N Engl J Med, 2016. 374(1): p. 13-22.

42. Brosens, I., et al., The "Great Obstetrical Syndromes" are associated with disorders of deep placentation. Am J Obstet Gynecol, 2011. 204(3): p. 193-201.

43. Crovetto, F., et al., First-trimester screening with specific algorithms for early- and lateonset fetal growth restriction. Ultrasound Obstet Gynecol, 2016. 48(3): p. 340-8.

44. Hendrix, M.L.E., et al., Reduced fetal growth velocities and the association with neonatal outcomes in appropriate-for-gestational-age neonates: a retrospective cohort study. BMC Pregnancy Childbirth, 2019. 19(1): p. 31.

45. Rolnik, D.L., et al., Aspirin versus Placebo in Pregnancies at High Risk for Preterm Preeclampsia. N Engl J Med, 2017. 377(7): p. 613-622.

46. Mlynarczyk, M., et al., The clinical significance of an estimated fetal weight below the 10th percentile: a comparison of outcomes of $<5$ th vs 5th-9th percentile. Am J Obstet Gynecol, 2017. 217(2): p. 198 e1-198 e11.

47. Gordijn, S.J., et al., Consensus definition of fetal growth restriction: a Delphi procedure. Ultrasound Obstet Gynecol, 2016. 48(3): p. 333-9.

48. Masseyeff, R.F., Standardization of immunoassays. Ann Ist Super Sanita, 1991. 27(3): p. 427-36.

49. Stenman, U.H., Immunoassay standardization: is it possible, who is responsible, who is capable? Clin Chem, 2001. 47(5): p. 815-20.

50. Andersen, L.B., et al., Diagnosis of preeclampsia with soluble Fms-like tyrosine kinase 1/ placental growth factor ratio: an inter-assay comparison. J Am Soc Hypertens, 2015. 9(2): p. 86-96. 
51. Schiettecatte, J., et al., Multicenter evaluation of the first automated Elecsys sFlt-1 and PIGF assays in normal pregnancies and preeclampsia. Clin Biochem, 2010. 43(9): p. 768-70.

52. Nucci, M., et al., Maternal serum placental growth factor (PIGF) isoforms 1 and 2 at 11-13 weeks' gestation in normal and pathological pregnancies. Fetal Diagn Ther, 2014. 36(2): p. 106-16.

53. van Helden, J. and R. Weiskirchen, Analytical evaluation of the novel soluble fms-like tyrosine kinase 1 and placental growth factor assays for the diagnosis of preeclampsia. Clin Biochem, 2015. 48(16-17): p. 1113-9.

54. McCarthy, F.P., et al., Comparison of three commercially available placental growth factor-based tests in women with suspected preterm pre-eclampsia: the COMPARE study. Ultrasound Obstet Gynecol, 2019. 53(1): p. 62-67. 


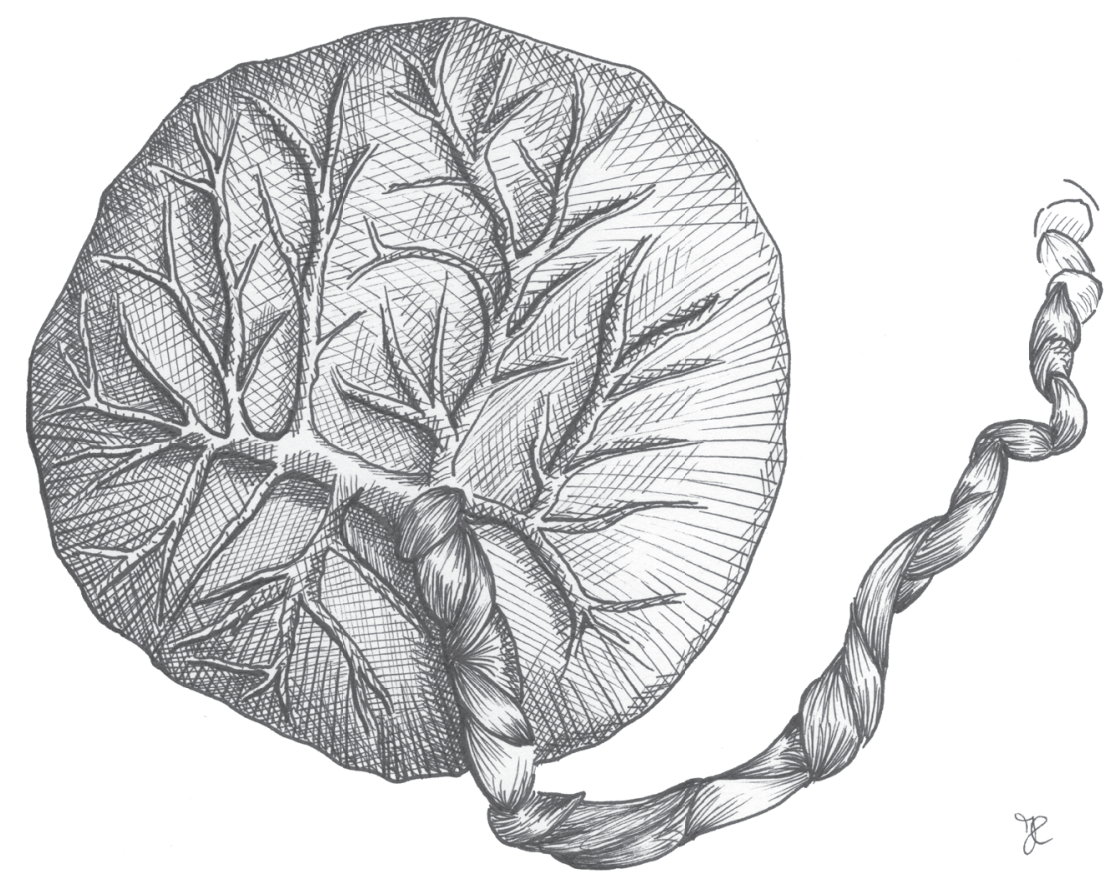


Maternal vascular malformation in the placenta is an indicator for fetal growth restriction irrespective of neonatal birthweight.

MLE Hendrix

JAP Bons

NO Alers

CAH Severens-Rijvers

MEA Spaanderman

S Al-Nasiry

Placenta. 2019 Nov;87:8-15 


\section{ABSTRACT}

\section{Introduction}

To study the association between placental pathology and neonatal birthweight and outcomes, and whether a combination of first trimester biomarkers and fetal growth velocity can predict placental lesions.

\section{Methods}

The presence of maternal vascular malperfusion (MVM) lesions (Amsterdam Criteria) was recorded in a retrospective cohort of singleton pregnancies in the Maastricht University Medical Centre, 2011-2018. First trimester maternal characteristics and PAPP-A, PIGF and sFlt-1 levels were collected. Fetal growth velocities were calculated (mm/week) from 20 to 32 week for abdominal circumference, biparietal diameter, head circumference and femur length. Data were compared between neonates with 'small for gestational age' (SGA < p10) and different categories of 'appropriate for gestational age (AGA)': AGA p10-30, AGA p30-50 and AGA > p50 (reference), using one-way ANOVA and post hoc test.

\section{Results}

There were significantly more MVM lesions in the SGA group $(94.6 \% \mathrm{p}<.0001)$, but also in the AGAp10-30 (67.3\%, p <.0001) and AGAp30-50 (41.6\% p=0.002), compared to the reference AGA group (19.3\%). The prediction of MVM for a $20 \%$ false-positive rate, with maternal characteristics was $25.2 \%$. The addition of birthweight percentile gave a prediction of 51.7\% for MVM. However adding placental biomarkers and fetal growth velocities (instead of birthweight percentile) to the maternal characteristics, gave a prediction of $81.8 \%$ (PPV 49.5\%, NPV 53.7\%).

\section{Discussion}

Placental MVM lesions correlated inversely with birthweight even in AGA neonates, and were associated with slower fetal growth and more adverse outcome in SGA neonates. A combination of first trimester biomarkers and fetal growth velocity had good prediction of placental MVM lesions, as an indicator of fetal growth restriction irrespective of neonatal weight. 


\section{INTRODUCTION}

Adequate fetal growth is a prerequisite for a successful pregnancy and for longterm health and well-being of the offspring. When fetal growth fails to meet its genetically predetermined potential, a pathological condition, fetal growth restriction (FGR) ensues [1, 2]. FGR complicates up to 3-6\% of all pregnancies and is a major obstetric problem causing significant perinatal morbidity and mortality [3-5]. Whilst FGR can occur as a direct consequence of a maternal disease or a fetal anomaly, the majority of FGR is caused by placental dysfunction [6]. Placental dysfunction results in insufficient supply of oxygen and nutrients to the fetus, which, if prolonged, leads to restriction of its growth and, ultimately in reduced size. Due to lack of consensus on what constitutes normal intrauterine growth, estimated fetal weight is used as proxy. Hence, growth restriction is most commonly defined as estimated fetal weight (EFW) or birthweight below the $10^{\text {th }}$ percentile, also called fetal growth restriction (FGR) or small-for-gestational age (SGA), respectively $[7,8]$. Conversely, a fetus that grows or has a birthweight above the $10^{\text {th }}$ percentile is considered to be appropriate-for-gestational age (AGA). However, it is conceivable that a number of AGA fetuses have decreased growth velocity during second and third trimester but not severe enough to cause smallness, and they, thereby, fall outside of the contemporary definition of FGR. These "relatively" growth restricted fetuses remain unrecognized, receive suboptimal monitoring, and are consequently at increased risk of morbidity and mortality [9, 10]. We and others have recently published on the added value of measuring fetal growth velocity in the diagnosis and management of late fetal growth restriction [11]. Reduced fetal growth velocity is currently part of the consensus definition of late fetal growth restriction.

The mechanism underlying the late mild phenotype of fetal growth restriction is still not fully understood. It is possible that this phenotype is associated with a milder placental disease, resulting from a disbalance in the production and secretion of angiogenic factors in the placenta. Angiogenic factors such as placental growth factor (PIGF) and its inhibitor, the anti-angiogenic soluble fms-like tyrosine kinase-1 (sFlt-1) are believed to be a key regulator of placental angiogenesis and vasculogenesis $[12,13]$. They are secreted in a balanced way by villous syncytiotrophoblast into the maternal circulation in response to hypoxic stimuli in the first trimester [14]. The balance of the pro- and anti-angiogenic effects of these biomarkers on maternal endothelial cell and leukocyte functions is essential for normal vascular adaptation of pregnancy and its dysregulation is well-described in preeclampsia [15]. Alternatively, PlGF is also secreted by extravillous trophoblast, as well as the endometrial epithelial cells and leukocytes 
at the fetomaternal interface indicating the potential modulatory functions during implantation and maternal spiral artery remodeling [16]. Low maternal serum levels of PIGF and high levels of sFlt-1 are seen in FGR foetuses and their levels are linked to the severity of growth restriction and placental pathology [17-21]. However, how the dysregulated angiogenic balance is linked to placental pathology in cases of FGR is not well understood [22].

Placental lesions characteristic of maternal vascular malperfusion (MVM) are thought to be the consequence of this process of defective remodelling of maternal spiral arteries, and are considered the placental hallmark of FGR [23, 24]. The birth of an FGR neonate in the first pregnancy increases the risk of a recurrence FGR by more than sevenfold [25]. However, in a cohort of 96 recurrent FGR pregnancies, Levy et al. showed that maternal vascular malperfusion (MVM) lesions and adverse neonatal outcomes were more prevalent in the first FGR pregnancy that in the subsequent FGR delivery [26]. The Amsterdam consensus on the definition of various placental characteristics and lesions provide a reliable and extensive framework to study the placental abnormalities associated with growth restriction [27].

In this study, we investigated if lower birthweight, even if considered appropriate for gestational age (AGA), is correlated with MVM and various other placental lesions. Additionally, we studied whether MVM lesions are more strongly predicted by a model using birthweight percentiles or a combination of angiogenic biomarkers and fetal growth velocities.

\section{METHODS}

\section{Study population}

This was a retrospective cohort study of singleton pregnancies delivered between January 2011 and August 2018 in Maastricht University Medical Centre, a tertiary academic centre in the Netherlands. Pregnant women with a fetus with congenital anomalies were excluded from the study. The study protocol was approved by the medical ethical committee of the Maastricht University Medical Center (17-40.15.1/ab). Procedures followed were in accordance with institutional guidelines and adhered to the principles of the Declaration of Helsinki and Title 45, U.S. Code of Federal Regulations, Part 46, Protection of Human Subjects (revised 13 November 2001, effective 13 December 2001). 


\section{Data collection and definitions}

During routine hospital visits, baseline data on maternal health were collected and recorded. These baseline data include maternal age, data on length, weight, smoking status, general medical and obstetric history and relevant information about the current pregnancy. Body mass index (BMI) was calculated by weight (kg) divided by height (m)-squared. Preeclampsia (PE) was defined according to the guidelines of International society for the Study of Hypertension in Pregnancy [28]. In normotensive women at baseline, PE was defined as the development of hypertension with proteinuria. When a woman was suspected of developing hypertension, blood pressure was recorded for a period of $30 \mathrm{~min}$ at 3-min intervals using a semiautomatic oscillometric device in half-sitting position. Median values of 9 subsequent recordings were used for analysis. Gestational hypertension was then defined as a median systolic blood pressure equal to or greater than 140 $\mathrm{mmHg}$, and/or a diastolic blood pressure equal or greater than $90 \mathrm{mmHg}$. The diagnosis of proteinuria required excretion of albumin/creatinine ratio of more than $30 \mathrm{mg} / \mathrm{mmol}$, without evidence of a urinary tract infection. The neonatal birtweight was calculated in percentiles according to the Dutch reference standard [29].

Based on birthweight four separate study groups were formed, namely birthweight $<10^{\text {th }}$ percentile (considered SGA), birthweight between the $10-30^{\text {th }}$ centile, birthweight between the $30-50^{\text {th }}$ centile and birthweight above the $50^{\text {th }}$ centile (without preeclampsia, hypertensive disease or diabetes), as reference group.

An experienced sonographist, who was not otherwise involved in the study, took fetal biometric measurements on a GE Voluson with a 2-5MHz curved-array transducer, in accordance with the routine mid-trimester fetal ultrasound scan guidelines [30]. Fetal biometry included measurement of the abdominal circumference (AC), biparietal diameter (BPD), head circumference (HC) and femur length (FL). Fetal growth scans were performed twice, one between 18 and 22 weeks of gestational age and one between 30 and 34 weeks gestational age as a routine third trimester growth scan [10,31]. Fetal growth parameters were recorded in an electronic database (Astraia, GMBH). Estimated fetal weight was calculated using the Hadlock equation [32] [Hadlock C; $\log (10) \mathrm{BW}=1.335-0.0034(\mathrm{AC})(\mathrm{FL})+$ $0.0316(\mathrm{BPD})+0.0457(\mathrm{AC})+0.1623(\mathrm{FL})]$. Growth velocities were calculated as the difference in absolute value of the measured fetal growth parameters between the two examination periods, divided by the number of weeks ( $\mathrm{mm} /$ week)[33]. If multiple scans were performed, the scan with a scan date which was closest to 20 weeks with a maximum range of 18 to 22 weeks or closest to 32 weeks with a maximum range of 30 to 34 weeks of gestational age was selected for analysis. 


\section{Sampling of blood serum}

Blood samples were collected at week 11-13 of gestational age as part of routine patient care and an extra serum sample was stored at -80 degrees until further analysis. Placental biomarkers (PIGF, sFlt-1, PAPP-A, and $\beta$-hCG) were retrospectively measured in serum. Samples were analysed in a certified laboratory using commercially available kits. Measurements of sFlt-1 and PIGF assays were performed according to the manufacturer's instructions on the fully automated BRAHMS KRYPTOR compact PLUS system (ThermoFisher Scientific, Hennigsdorf, Germany). Both assays are immunometric assays based on the Time Resolved Amplified Cryptate Emission (TRACE) technology as described elsewhere [34]. Measurements of PAPP-A and free $\beta$-hCG were performed according to the manufacturer's instructions on the (fully automated) Auto-Delfia system (PerkinElmer,Turku, Finland). Both assays are fluoroimmunometric assays.

\section{Neonatal outcome data}

All data regarding delivery and neonatal outcome were registered by an obstetrician or paediatrician. Apgar scores of the newborn were assessed at 1 and 5 minutes after birth. The cut-off point for adverse outcome was an Apgar-score less than 7 at 5 minutes after birth. NICU admissions were reported and a composite adverse neonatal outcome consisting of asphyxia, sepsis, respiratory distress syndrome [35] and transient tachypnea [36] was used. All of these complications were recorded in the medical files. Asphyxia was diagnosed if the new-born suffered from intrapartum-related hypoxia-ischemia with multiple organ failure including encephalopathy [37]. Stillbirth and postnatal death during hospitalization were recorded.

\section{Placental histology evaluation}

Placental histological examinations were performed according to a local standard protocol. The pathologist was blinded for dependent and independent variables, like birthweight percentile categories, fetal growth velocities, and biomarker results. The pathologist was not blinded for gestational age and absolute birthweight outcome. After delivery, placentas were placed in $4 \%$ buffered formalin and allowed to fix for at least 48 hours. The umbilical cord length and diameter were measured and the cord was inspected. In particular, insertion site, number of vessels and number of coils were recorded. The implantation was documented as (para)central, marginal (within $1 \mathrm{~cm}$ of the placental disc margin) or velamentous. The umbilical coiling index (UCI) was determined by dividing the total number of coils by the total umbilical cord length in centimeters. Hypocoiling was defined as an $\mathrm{UCI}<0.1$ and hypercoiling as an UCI $>0$.3. Placental membranes 
were inspected as well and both the cord and membranes were removed from the placental parenchym. The placental disc was weighed after fixation, without the umbilical cord and placental membranes. Placental weight was compared to reference values for the corresponding gestational age [38]. The placental disc was measured in three dimensions. Subsequently, the placental disc was cut in $1 \mathrm{~cm}$ thick slices and the cut surface was grossly inspected. Any macroscopic lesions were documented and sampled, along with standard samples. Standard samples include two cross-sections of the umbilical cord (one at the fetal end and one section at the maternal end), two membrane roll sections and two full-thickness sections of normal appearing placental parenchyme within the central two thirds of the placental disc. The samples were embedded in paraffin and cut into $4 \mu \mathrm{m}$ thick slides. The slides were stained with H\&E (hematoxylin and eosin) and assessed by a pathologist. Findings were documented in the pathology report which was added to the electronic patient record.

Maternal vascular malperfusion (MVM) was diagnosed when certain gross and microscopic findings are present in de placenta. Criteria for gross findings were placental hypoplasia, infarction of more than $5 \%$ and retroplacental haemorrhage. Criteria for microscopic findings are distal villous hypoplasia, accelerated villous maturation and decidual arteriopathy (including acute atherosis) [27].

Histological microscopic placental findings are noted and included different abnormalities. Infarction was divided into three categories according to affected percentage in relation to total placental parenchym (infarct $<5 \%$, infarct $5-15 \%$ and infarct 15-30\%). Percentages are calculated macroscopically, by determining the percentage of parenchymatous lesions on cut section to the nearest 5 percent. The lesions are then sectioned for microscopic evaluation. If there are different types of microscopic lesions, for instance both infarction and intervillous thrombus, the percentage is adjusted for the degree of infarction (again the nearest 5 percent). Haematoma was noted according to the location. Other findings included thrombosis and avascular villi. Maternal floor infarct was defined as massive perivillous fibrin depositions. Inflammation of the membranes was divided into different categories; inflammation of the decidua, inflammation of the decidua and chorion, inflammation of the decidua, chorion and the amnion.

\section{Statistical analysis}

Differences between two groups were tested using the independent T-test for continuous data. Results were presented as mean and standard deviation (SD). Differences between three or more groups were calculated with ANOVA and the bonferroni post hoc test. For comparing categorical data between groups, the chi-square test was performed. 
Potential predictors of MVM were tested in three models. The first model includes the maternal characteristics: maternal age, conception method (spontaneous vs assisted), smoking during pregnancy (yes/no), maternal BMI. The second model consists of the maternal characteristics (first model) and birthweight percentile. The third model consists of maternal characteristics, serum PlGF, sFlt-1 and sFlt-1/ PlGF ratio measured at 12 weeks of gestational age and fetal growth velocities (mm/week) of abdominal circumference (ACv), biparietal diameter (BPDv), head circumference $(\mathrm{HCv})$ and femur length (FLv) between 20 and 32 weeks of gestational age.

\section{RESULTS}

\section{General characteristics}

Between January 2011 and August 2018, we included 394 women with a singleton pregnancy. The reference group, birthweight $>50^{\text {th }}$ percentile $(n=115)$, the AGA $30-50^{\text {th }}$ percentile $(n=126)$, AGA $10-30^{\text {th }}$ percentile $(n=113)$ and the SGA group $(n=37)$.

The general characteristics of the study population are represented in Table 1. There were no significant differences for maternal age, BMI and primiparous. In the AGA30-50 group 16.2\% of the women smoked, 27.5\% in the AGA10-30 group and $17.4 \%$ of the SGA group, compared with $17.6 \%$ in the reference group. Diabetes was present in 6.3\% in the AGA30-50 group and 6.2\% in the AGA10-30 group, while it was absent in the SGA group. PE was diagnosed in $11.1 \%$ of all the pregnancies in the AGA30-50 group, in 23.9\% of the AGA10-30 group and in $29.7 \%$ of the SGA group. In the AGA30-50 group 23.4\% was diagnosed with gestational hypertension, compared to $50.4 \%$ in the AGA10-30 group and $57.1 \%$ in the SGA group. Gestational age at birth was significantly lower in the SGA group (217.82 25.96 , $\mathrm{p}<.0001)$ compared to the reference group $(247.54 \pm 26.86)$. There were no significant differences in gestational age at birth between the reference group and AGA10-30 and AGA30-50 groups.

\section{Neonatal outcome data}

Table 2 shows neonatal outcomes in all study groups. There were no significant differences in the percentage of neonates with Apgar $<7$ at 5 minutes, postnatal death or stillbirths between all groups. However, there were significantly more NICU admissions in the SGA group $(82.4 \%, \mathrm{p}<.0001)$ compared to the reference group (33\%). SGA fetuses were diagnosed with an adverse neonatal outcome significantly more often than the reference group (45.9\% versus $12.2 \%, \mathrm{p}<.0001$ ). 
Table 1. Maternal and pregnancy baseline characteristics, birthweight percentiles (bwp) $>50$ (reference group) versus bwp30-50, bwp10-30 and bwp $<10$.

\begin{tabular}{|c|c|c|c|c|c|c|c|}
\hline & $\begin{array}{c}\text { Birthweight } \\
\text { percentile } \\
>50 \\
(n=115)\end{array}$ & $\begin{array}{c}\text { Birthweight } \\
\text { percentile } \\
30-50 \\
(\mathrm{n}=126)\end{array}$ & P-value & $\begin{array}{c}\text { Birthweight } \\
\text { percentile } \\
10-30 \\
(n=113)\end{array}$ & P-value & $\begin{array}{c}\text { Birthweight } \\
\text { percentile } \\
<10 \\
(\mathrm{n}=37)\end{array}$ & P-value \\
\hline Maternal age (years) & $\begin{array}{c}31.3 \pm 4.9 \\
(20-43)\end{array}$ & $\begin{array}{c}31.0 \pm 4.7 \\
(19-44)\end{array}$ & 1.000 & $\begin{array}{c}30.4 \pm 5.2 \\
(18-43)\end{array}$ & 1.000 & $\begin{array}{c}30.3 \pm 4.4 \\
(20-39)\end{array}$ & 1.000 \\
\hline $\operatorname{BMI}\left(\mathrm{kg} / \mathrm{m}^{2}\right)$ & $\begin{array}{c}27.9 \pm 5.9 \\
(18-46)\end{array}$ & $\begin{array}{c}27.5 \pm 6.6 \\
(17-47)\end{array}$ & 1.000 & $\begin{array}{c}26.5 \pm 5.7 \\
(17-43)\end{array}$ & 0.305 & $\begin{array}{c}26.8 \pm 5.1 \\
(19-38)\end{array}$ & 1.000 \\
\hline Primiparous (\%) & $50.4(58 / 115)$ & $46.8(59 / 126)$ & 0.416 & $57.5(65 / 113)$ & 1.000 & $67.6(25 / 37)$ & 1.000 \\
\hline \multicolumn{8}{|l|}{ Smoking } \\
\hline Never (\%) & $71.4(65 / 91)$ & 79.8 (79/99) & 0.217 & $69.2(63 / 91)$ & 0.005 & $65.2(15 / 23)$ & 0.024 \\
\hline Stopped (\%) & $10.0(10 / 91)$ & $4.0(4 / 99)$ & 0.077 & $3.3(3 / 91)$ & 0.036 & $17.4(4 / 23)$ & 0.500 \\
\hline Yes (\%) & $17.6(16 / 91)$ & $16.2(16 / 99)$ & 1.000 & $27.5(25 / 91)$ & 0.351 & $17.4(4 / 23)$ & 1.000 \\
\hline Diabetes (\%) & - & $6.3(8 / 126)$ & 0.010 & $6.2(7 / 113)$ & 0.016 & $0(0 / 37)$ & 1.000 \\
\hline $\mathrm{PE}(\%)$ & - & $11.1(14 / 126)$ & $<.0001$ & $23.9(27 / 113)$ & $<.0001$ & $29.7(11 / 37)$ & $<.0001$ \\
\hline Hypertension (\%) & - & $23.4(29 / 124)$ & $<.0001$ & $50.4(57 / 113)$ & $<.0001$ & $57.1(20 / 35)$ & $<.0001$ \\
\hline \multirow[t]{2}{*}{ GA (days) } & $\begin{array}{c}247.5 \pm 26.9 \\
(174-288)\end{array}$ & $\begin{array}{c}243.2 \pm 31.8 \\
(176-291)\end{array}$ & 1.000 & $\begin{array}{c}241.8 \pm 29.2 \\
(175-291)\end{array}$ & 1.000 & $\begin{array}{c}217.8 \pm 26.0 \\
(189-279)\end{array}$ & $<.0001$ \\
\hline & $32.2(37 / 115)$ & $38.1(48 / 126)$ & & $40.7(46 / 113)$ & & $75.7(28 / 37)$ & \\
\hline GA <34 weeks (\%) & $21.7(25 / 115)$ & $14.3(18 / 126)$ & 1.000 & $12.4(14 / 113)$ & 1.000 & $13.5(5 / 37)$ & $<.0001$ \\
\hline GA 34-36 weeks (\%) & $46.1(53 / 115)$ & $47.6(60 / 113)$ & 0.685 & $46.9(53 / 113)$ & 0.324 & $10.8(4 / 37)$ & 1.000 \\
\hline GA > 36 weeks (\%) & & & 1.000 & & 1.000 & & 0.001 \\
\hline Birthweight (g) & $\begin{array}{c}2661 \pm 857.7 \\
(810-4350)\end{array}$ & $\begin{array}{c}2270 \pm 851.5 \\
(680-3510)\end{array}$ & 0.002 & $\begin{array}{c}1998.1 \pm 786.4 \\
(620-3420)\end{array}$ & $<.0001$ & $\begin{array}{c}1122 \pm 569.8 \\
(638-2694)\end{array}$ & $<.0001$ \\
\hline $\begin{array}{l}\text { Birthweight } \\
\text { percentile }\end{array}$ & $\begin{array}{c}68.0 \pm 11.8 \\
(51-100)\end{array}$ & $\begin{array}{c}40.1 \pm 5.9 \\
(30-50)\end{array}$ & 0.000 & $\begin{array}{c}20.4 \pm 5.5 \\
(10-29)\end{array}$ & $<.0001$ & $\begin{array}{c}5.3 \pm 2.7 \\
(0-9)\end{array}$ & $<.0001$ \\
\hline
\end{tabular}

Data are given as mean $\pm S D$ (min-max) or \% $(\mathrm{n} / \mathrm{N})$. BMI: body mass index $\left(\mathrm{kg} / \mathrm{m}^{2}\right)$; PE: preeclampsia; GA: gestational age.

Table 2. Neonatal outcome of the study groups, birthweight percentile (bwp) $>50$ (reference group) versus bwp30-50, bwp10-30 and bwp $<10$.

\begin{tabular}{|c|c|c|c|c|c|c|c|}
\hline & $\begin{array}{c}\text { Birthweight } \\
\text { percentile } \\
>50 \\
\text { (reference) } \\
(\mathrm{n}=115)\end{array}$ & $\begin{array}{c}\text { Birthweight } \\
\text { percentile } \\
30-50 \\
(n=126)\end{array}$ & P-value & $\begin{array}{c}\text { Birthweight } \\
\text { percentile } \\
10-30 \\
(n=113)\end{array}$ & P-value & $\begin{array}{c}\text { Birthweight } \\
\text { percentile } \\
<10 \\
(\mathrm{n}=37)\end{array}$ & P-value \\
\hline APGAR 5min <7 (\%) & $13.0(15 / 115)$ & $11.9(15 / 126)$ & 0.788 & $8.0(9 / 113)$ & 0.205 & $10.8(4 / 37)$ & 0.711 \\
\hline NICU stay & $33.0(38 / 115)$ & $42.0(50 / 126)$ & 0.286 & $42.3(47 / 113)$ & 0.184 & $82.4(28 / 37)$ & $<.0001$ \\
\hline Adverse neonatal outcome (\%) & $12.2(14 / 115)$ & $23.8(30 / 126)$ & 0.018 & $23.0(26 / 113)$ & 0.032 & $45.9(17 / 37)$ & $<.0001$ \\
\hline Neonatal death (\%) & $0.9(1 / 115)$ & $0.8(1 / 126)$ & 0.949 & $1.8(2 / 113)$ & 0.554 & $0(0 / 37)$ & 0.319 \\
\hline Stillbirth (\%) & $2.6(3 / 115)$ & $0.8(1 / 126)$ & 0.285 & $0(0 / 113)$ & 0.083 & $2.7(1 / 37)$ & 0.975 \\
\hline
\end{tabular}

Data are given as \% (n/N). NICU: neonatal intensive care unit. Composite adverse neonatal outcome consists of 4 complications: asphyxia, sepsis, respiratory distress syndrome and transient tachypnoea of the newborn. Asphyxia: intrapartum-related hypoxia-ischemia with multiple organ failure including encephalopathy. Neonatal death: first 28 days of life . 
Also, neonates with a birthweight percentile 30-50 had more adverse neonatal outcome than the reference group ( $23.8 \%$ versus $12.2 \%, \mathrm{p}=0.018)$.

\section{Differences in placental lesions}

Table 3 and figure 1 show the differences in placental lesions between the categories of birthweight percentiles. There was a significant trend between birthweight percentile and the existence of MVM lesions ( $\mathrm{F}=89.1, \mathrm{p}<.0001)$, distal villous hyoplasia $(\mathrm{F}=8.2, \mathrm{p}=0.004)$, accelerated villous maturation $(\mathrm{F}=55.9, \mathrm{p}=<.0001)$, infarct $(\mathrm{F}=38.4, \mathrm{p}=<.0001)$ and placental hypoplasia $(\mathrm{F}=60.8, \mathrm{p}=<.0001)$.

There were significantly more MVM lesions in the AGA30-50 (41.6\%, $\mathrm{p}=0.002$ ), AGA10-30 (67.3\%, p<.0001) and SGA group (94.6\%, p<.0001), compared with the reference AGA group (19.3\%). The different sub-lesions belonging to MVM were compared separately as well. Distal villous hypoplasia was significantly more common in the SGA group $(8.1 \%, \mathrm{p}=0.025)$ compared to the reference group $(0 \%)$. There was no significant difference in the AGA10-30 and AGA30-50 versus the reference group. Accelerated villous maturation however was significantly higher compared to the reference group (5.2\%), with 25.7\% in the AGA10-30 ( $<<.0001)$ and 59.5\% in the SGA group ( $\mathrm{p}<.0001)$. AGA10-30 $(12.4 \%, \mathrm{p}=0.001)$ and SGA $(27.0 \%$, $\mathrm{p}<.0001)$ were diagnosed significantly more often with infarct $(>5 \%)$ compared to the reference group (1\%). Hypoplasia of the placenta was also significantly higher in both the AGA10-30 group (43.4\%, $\mathrm{p}<.0001)$ and the SGA group $(67.6 \%, \mathrm{p}<.0001)$ compared to the reference group (11.3\%). Vasculopathy was significantly more common in the AGA10-30 group and SGA group with respectively $17.7 \%(\mathrm{p}=0.026)$ and $27 \%(\mathrm{p}=0.003)$.

The placentas of the AGA30-50 group were significantly lower in weight compared to the reference group (394.86 \pm 141.97 vs $441.76 \pm 128.86, \mathrm{p}=0.038)$. The same trend was seen in the AGA10-30 group (344.11 \pm 112.57 vs $441.76 \pm 128.86$, $\mathrm{p}<.0001)$ and in the SGA group $(181.93 \pm 47.42, \mathrm{p}<.0001)$

The placenta's showed significantly higher total infarct rates in the AGA10-30 group (40.7\%, p=0.001) and the SGA group (59.5\%, $\mathrm{p}<.0001)$ compared to the reference group (18.3\%). The SGA group showed more avascular villi in the placenta, with a percentage of $10.8 \%$ compared to $1.7 \%$ in the reference group $(\mathrm{p}=<.0001)$.

There were no significant differences between all groups in the categories of haematoma, inflammation of the membranes, maternal floor infarcts, delayed villous maturation, umbilical coil index, hyper- or hypo coiling, marginal or velamentous insertion or having a single umbilical artery. 
Table 3. Placental characteristics of the different birthweight percentile groups.

\begin{tabular}{|c|c|c|c|c|c|c|c|}
\hline & $\begin{array}{c}\text { Birthweight } \\
\text { percentile } \\
>50 \\
(n=115)\end{array}$ & $\begin{array}{c}\text { Birthweight } \\
\text { percentile } \\
30-50 \\
(n=126)\end{array}$ & P-value & $\begin{array}{c}\text { Birthweight } \\
\text { percentile } \\
10-30 \\
(n=113)\end{array}$ & P-value & $\begin{array}{c}\text { Birthweight } \\
\text { percentile } \\
<10 \\
(n=37)\end{array}$ & P-value \\
\hline \multicolumn{8}{|c|}{ Macroscopic placenta characteristics } \\
\hline Placenta weight (g) & $\begin{array}{c}441.8 \pm 128.9 \\
(188-784)\end{array}$ & $\begin{array}{c}394.9 \pm 142.0 \\
(128-700)\end{array}$ & 0.038 & $\begin{array}{c}344.11 \pm 112.57 \\
(103-610)\end{array}$ & $<.0001$ & $\begin{array}{c}181.9 \pm 47.4 \\
(125-253)\end{array}$ & $<.0001$ \\
\hline Placental length (cm) & $\begin{array}{l}17.7 \pm 3.1 \\
(7.5-26.5)\end{array}$ & $\begin{array}{c}16.8 \pm 3.0 \\
(7-23.5)\end{array}$ & 0.770 & $17.0 \pm 2.6(11-24)$ & 1.000 & $\begin{array}{c}14.3 \pm 2.3 \\
(11-17)\end{array}$ & $<.0001$ \\
\hline Placental width (cm) & $\begin{array}{c}15.1 \pm 2.3 \\
(9-20)\end{array}$ & $\begin{array}{c}15.1 \pm 3.0 \\
(9-21)\end{array}$ & 1.000 & $14.1 \pm 2.5(7-21)$ & 0.054 & $\begin{array}{c}11.9 \pm 1.7 \\
(10-15)\end{array}$ & $<.0001$ \\
\hline $\begin{array}{l}\text { Placental thickness } \\
(\mathrm{cm})\end{array}$ & $2.5 \pm 0.9(1-6)$ & $\begin{array}{c}2.5 \pm 0.8 \\
(1-5)\end{array}$ & 1.000 & $2.4 \pm 1.3(2-5)$ & 1.000 & $\begin{array}{c}2.0 \pm 0.6 \\
(2-3)\end{array}$ & 0.002 \\
\hline \multicolumn{8}{|c|}{ Macroscopic umbilical cord characteristics } \\
\hline $\begin{array}{l}\text { Umbilical coiling } \\
\text { index }\end{array}$ & $\begin{array}{c}0.2 \pm 0.12 \\
(0.0-0.7)\end{array}$ & $\begin{array}{r}0.2 \pm 0.2 \\
(0.1-0.9)\end{array}$ & 0.638 & $0.2 \pm 0.1(0.0-0.7)$ & 1.000 & $\begin{array}{l}0.3 \pm 0.2 \\
(0.1-0.5)\end{array}$ & 0.187 \\
\hline Hypercoiling (\%) & $20.0(14 / 70)$ & $20.8(16 / 77)$ & 0.908 & $19.1(13 / 68)$ & 0.070 & $46.2(6 / 13)$ & 0.106 \\
\hline Hypocoiling (\%) & $11.4(8 / 70)$ & $10.4(8 / 77)$ & 0.841 & $13.2(9 / 68)$ & 0.749 & $8.3(1 / 12)$ & 0.755 \\
\hline $\begin{array}{l}\text { Marginal or } \\
\text { velamentous } \\
\text { insertion (\%) }\end{array}$ & $25.5(12 / 85)$ & $20.7(23 / 111)$ & 0.225 & $16.5(16 / 97)$ & 0.658 & $17.1(6 / 35)$ & 0.676 \\
\hline $\begin{array}{l}\text { Single umbilical } \\
\text { artery (\%) }\end{array}$ & $3.1(3 / 98)$ & $3.8(4 / 104)$ & 0.762 & $1.1(1 / 91)$ & 0.352 & $5.6(1 / 18)$ & 0.598 \\
\hline \multicolumn{8}{|c|}{ Microscopic characteristics } \\
\hline Infarct (\%) & $21.4(21 / 98)$ & $27.8(35 / 126)$ & 0.212 & $40.7(46 / 113)$ & 0.001 & $59.5(22 / 37)$ & $<.0001$ \\
\hline Infarct <5\% (\%) & $95.2(20 / 21)$ & $91.4(32 / 35)$ & 0.600 & $68.9(31 / 45)$ & 0.003 & $54.4(12 / 22)$ & 0.002 \\
\hline Infarct 5-15\% (\%) & $4.8(1 / 21)$ & $5.7(2 / 35)$ & 0.881 & $22.2(10 / 45)$ & 0.030 & $40.9(9 / 22)$ & 0.005 \\
\hline Infarct $15-30 \%$ (\%) & $0.0(0 / 21)$ & $2.9(1 / 35)$ & 0.444 & $6.7(3 / 45)$ & 0.083 & $4.5(1 / 22)$ & 0.329 \\
\hline Haematoma (\%) & $4.2(4 / 96)$ & $10.8(11 / 102)$ & 0.079 & $3.6(3 / 84)$ & 0.838 & $18.8(3 / 16)$ & 0.206 \\
\hline Thrombosis (\%) & $20.6(21 / 102)$ & $25.7(27 / 105)$ & 0.526 & $18.6(16 / 86)$ & 0.603 & $44.4(8 / 18)$ & 0.074 \\
\hline \multicolumn{8}{|l|}{$\begin{array}{l}\text { Inflammation } \\
\text { membranes }\end{array}$} \\
\hline -deciduitis (\%) & $18.3(21 / 115)$ & $19.8(25 / 126)$ & 0.927 & $61.5(16 / 26)$ & 0.235 & $2.7(1 / 37)$ & 0.647 \\
\hline -chorionitis (\%) & $7.0(8 / 115)$ & $6.3(8 / 126)$ & 0.124 & $2.7(3 / 113)$ & 0.891 & $0(0 / 37)$ & 0.479 \\
\hline -chorioamnionitis (\%) & $1.7(2 / 115)$ & $5.6(7 / 126)$ & 0.967 & $0.9(1 / 113)$ & 0.673 & $2.7(1 / 37)$ & 0.476 \\
\hline Calcification (\%) & $7.0(8 / 115)$ & $15.1(20 / 126)$ & 0.239 & $15.0(17 / 113)$ & 0.243 & $5.4(2 / 37)$ & 0.946 \\
\hline Avascular villi (\%) & $1.7(2 / 115)$ & $0.8(1 / 126)$ & 0.539 & $1.8(2 / 113)$ & 0.903 & $10.8(4 / 37)$ & 0.040 \\
\hline Oedema (\%) & $13.0(15 / 115)$ & $9.5(12 / 126)$ & 0.464 & $7.1(8 / 113)$ & 0.217 & $2.7(1 / 37)$ & 0.209 \\
\hline Fibrin deposition (\%) & $35.7(41 / 115)$ & $27.0(34 / 126)$ & 0.176 & $24.8(28 / 113)$ & 0.178 & $8.1(3 / 37)$ & 0.045 \\
\hline $\begin{array}{l}\text { Maternal floor infarct } \\
\text { (\%) }\end{array}$ & $0.0(0 / 115)$ & $0.8(1 / 126)$ & 0.339 & $0.9(1 / 113)$ & 0.320 & $0.0(0 / 37)$ & 1.000 \\
\hline $\begin{array}{l}\text { Villous maturation } \\
\text { Delayed (\%) }\end{array}$ & $1.7(2 / 115)$ & $3.4(4 / 126)$ & 0.425 & $6.2(7 / 113)$ & 0.067 & $2.7(1 / 37)$ & 0.321 \\
\hline Acute atherosis (\%) & $0.0(0 / 115)$ & $0.8(1 / 126)$ & 0.334 & $4.4(5 / 113)$ & 0.025 & $0.0(0 / 37)$ & 1.000 \\
\hline
\end{tabular}

Data are given as mean $\pm S D$ (min-max) or $\%(n / N)$. 


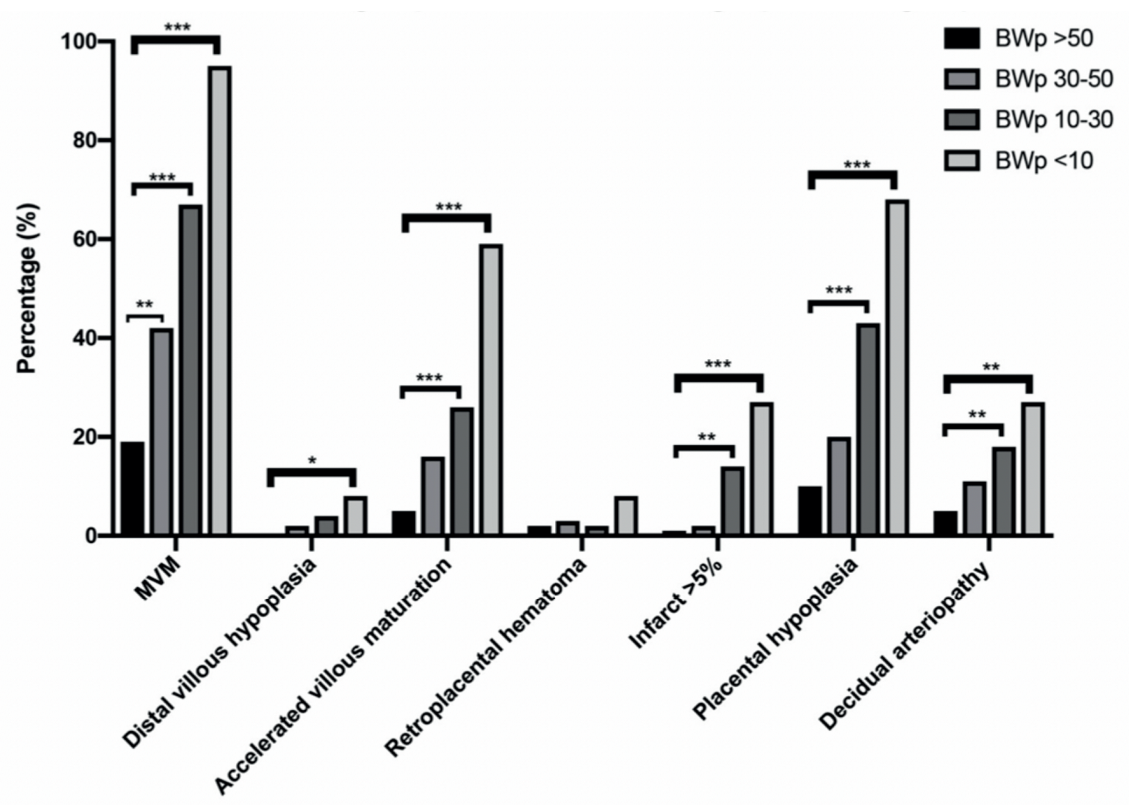

MVM lesions

Figure 1 Maternal vascular malperfusion lesions in different birth weight percentile groups.

\section{Predictive performance for MVM}

Three models were compared in the prediction of MVM. The discriminative ability of the three different models as quantified by AUC is shown in table 4 . For the prediction of MVM, the maternal characteristics were used in screening model 1 (maternal age, conception method, smoking during pregnancy and parity) and had an AUC of 0.539 (95\% CI 0.468-0.609). Adding birthweight percentile to the baseline screening (model 2), improved the prediction of MVM with an AUC of 0.695 (95\% CI 0.541-0.849). Model 3 consist of maternal characteristics, biomarkers (PlGF, sFlt-1 and PAPP-a) and growth velocities (abdominal circumference, biparietal diameter, head circumference, femur length velocity), this improved the prediction of MVM with an AUC of 0.855 (0.653-1.000). For a 20\% false-positive rate (FPR), model 1, maternal characteristics, predicted $25.2 \%$ of placental tissues with MVM lesions. The addition of birthweight percentile gave a prediction of $51.7 \%$ for MVM. However adding placental biomarkers and fetal growth velocities (instead of birthweight percentile) to the maternal characteristics, gave a prediction of $81.8 \%$ (PPV 49.5\%, NPV 53.7\%). 
Table 4. Predictive performance of three models for the prediction of MVM lesions.

\begin{tabular}{lccc}
\hline $\begin{array}{l}\text { Predictive } \\
\text { value }\end{array}$ & $\begin{array}{c}\text { Model 1 } \\
\text { Maternal characteristics }\end{array}$ & $\begin{array}{c}\text { Model 2 } \\
\text { MC + BWp }\end{array}$ & $\begin{array}{c}\text { Model 3 } \\
\text { MC + FGv + placental } \\
\text { biomarkers }\end{array}$ \\
\hline AUC & $0.539(0.468-0.609)$ & $0.695(0.541-0.849)$ & $0.855(0.653-1.000)$ \\
\hline 10\% FPR & $11.0(6.8-16.6)$ & $13.8(9.1-19.8)$ & $36.4(29.4-44.0)$ \\
\hline Sensitivity & $10.4(6.5-16.0)$ & $12.8(8.5-18.6)$ & $28.1(22.4-34.5)$ \\
\hline PPV & $10.3(6.4-16.0)$ & $10.6(6.6-16.5)$ & $13.8(8.6-21.3)$ \\
\hline NPV & $25.2(18.5-31.5)$ & & $81.8(75.5-87.4)$ \\
\hline 20\% FPR & $23.2(17.5-29.9)$ & $51.7(44.1-59.2)$ & $49.5(43.6-55.4)$ \\
\hline Sensitivity & $20.9(15.3-27.9)$ & $38.4(32.2-44.9)$ & $53.7(41.2-65.8)$ \\
\hline PPV & $29.8(22.0-38.8)$ & \\
\hline
\end{tabular}

Model 1: maternal characteristics (maternal age, conception method, smoking during pregnancy and parity). Model 2: maternal characteristics with birthweight percentile. Model 3: maternal characteristics with fetal growth velocity (FGv) between 20 and 32 weeks of pregnancy and placental biomarkers: PlGF, sFlt-1 and PAPP-A.

\section{DISCUSSION}

\section{Main findings}

The present study demonstrated that the negative correlation between birthweight and placental vascular pathology, as well as adverse neonatal outcome, is not confined to SGA neonates but is also present across all categories of birthweight of neonates considered appropriate for gestational age. The prediction of placental MVM lesions, a hallmark of placental dysfunction, was higher using a combination of fetal growth velocities and placental biomarkers, than using birthweight percentiles, suggesting that these ultrasound and blood markers are more related to the pathology of fetal growth restriction than neonatal smallness.

\section{Interpretation}

Previous research has mainly described the impact of placental vascular pathology on birthweight and adverse outcome in the SGA population, as the main risk group for placental insufficiency [24]. In this study, we focused in particular on AGA neonates subdivided into categories of birthweight percentiles to investigate the impact of placental lesions on the milder phenotype of FGR. There is little existing literature on the prevalence and influence of placental MVM lesions in AGA neonates, as most studies pooled AGA neonates into one whole group, which was subsequently used as a reference to the SGA group. Our finding of a stepwise increase in MVM lesions in lower birthweight categories of AGA neonates supports the hypothesis that there is a subgroup of placental-mediated FGR fetuses 
in neonates born as AGA. This challenges the existing paradigm of using neonatal size, and by extrapolation estimated fetal size, as the basis for defining fetal growth restriction. Our findings also highlight the importance of identifying the subgroup of "normal sized" FGR fetuses, as they pose a potential perinatal risk similar to the SGA group.

Despite advances in our understanding of the placental origins of fetal growth restriction [39] the exact contribution of placental lesions to the clinical diagnosis and associated morbidity of fetal growth restriction is not fully understood. It is important to discuss that the association between placental MVM lesions and neonatal morbidity is not a direct or causative one, but rather circumstantial as both are related to decreased birth weight percentile. Previous studies have shown that SGA pregnancies exhibited more MVM lesions compared to AGA pregnancies $[23,24,40]$. Only about a fifth of the SGA placentas were totally free of histological abnormalities, while this was the case in about three quarters of the AGA group [41]. After birth, SGA fetuses with MVM lesions were at greater risk of neurodevelopment problems compared to SGA fetuses without placental MVM lesions [42].

It has long been acknowledged that being born small is linked to increased mortality and morbidity, including respiratory distress or intraventricular hemorrhage, as well as lifelong implications [43]. This knowledge translated in clinical practice into a focused attention on monitoring FGR fetuses and the inadvertent negligence of the risks of placental dysfunction to the AGA fetuses [1]. Our results suggest that fetuses with birthweights as high as the $30-50^{\text {th }}$ centile also showed placental lesions characteristic of growth restricted babies and were at risk for adverse neonatal outcome. A recent study involving over 6000 infants confirmed that the majority of adverse perinatal outcomes (including stillbirth, low cord $\mathrm{pH}$, emergency caesarean delivery for fetal distress in labour and neonatal intensive care unit admission) occurred among AGA fetuses [1]. Our data add evidence to the emerging concept that the placental dysfunction causing FGR is not only restricted to SGA fetuses, but can also occur in different degrees in AGA fetuses [44, $45]$. We argue that antenatal recognition of FGR fetuses would remain suboptimal unless it incorporates some form of estimation of reduced fetal growth, in addition to fetal size. There is ongoing debate among researchers on whether to use a population-specific curve, a global standard for fetal growth or a customized chart [46]. Our previous research has shown, that incorporating fetal growth velocity, measured by relative change in fetal abdominal circumference over time, improves the prediction of neonates with adverse outcome [47]. Identifying these fetuses with the highest risks for perinatal morbidity is the first step towards focusing the resources for antenatal surveillance that utilizes measures of fetal 
hemodynamic adaptations to reduced placental function. Subsequently, women with suspected fetal growth restriction should be counselled about the optimal timing of delivery, which several guidelines have set at 37-38 weeks [48]. This recommendation is based on the findings of randomized controlled trial of 650 women with SGA > 36 weeks (DIGITAT study), in which women with expectant management had a 2 -fold increase in risk of developing preeclampsia compared to women who had induction of labour, despite no difference in the perinatal outcomes [49].

\section{Placental function}

The intricate mechanisms behind normal placental function are relevant to fetal growth, as they ensure continuous provision of fetal needs of nutrients and oxygen, the elimination of waste products and protection from pathogens. Dysfunction or injury to the placenta, disrupts these essential processes and can cause growth impairment and even stillbirth [50, 51]. The most common mechanism of injury to the placenta, is thought to be via hypoxia-reoxygenation insults to the developing placental villi early in pregnancy. This process is initiated by failed physiologic transformation of the spiral arteries, which results in restricted maternal blood flow into the intervillous space. Differences in oxygen tension will lead to oxidative stress and free radical damage to the placental villi, resulting in histologic features of maternal vascular malperfusion (MVM) [52-54]. These pathologic lesions are characteristic of severe early placental dysfunction, however, our data suggest that similar lesions develop in a more subtle and insidious manner over time leading to a milder form of FGR. In this study, accelerated villous maturation and placental hypoplasia, two typical MVM lesions seen in early FGR and preeclampsia [55], were more often seen in pregnancies with a decrease in birthweight in the AGA group.

The improved prediction of MVM lesions using sFlt-1 and P1GF levels has implications on our understanding of the role of placental angiogenic biomarkers in the pathogenesis of FGR. First, PIGF is suggested to play a role in maternal spiral artery remodeling since it has been expressed by key regulators of this process at the fetomaternal interface including invading extravillous trophoblasts, endometrial epithelial cells and endometrial leukocytes [16]. This would suggest that aberrant production of pro-angiogenic signals is primarily involved in defective spiral artery remodeling leading to placental ischaemic injury and secondary development of MVM lesions. Alternatively, it is more plausible to think of reduced pro-angiogenic factors as a consequence of defective spiral artery remodeling, rather than its cause, and that both disturbed placental angiogenesis and MVM are downstream effects of the ensuing placental ischemia [22]. Previous research 
has shown that in placental tissues with MVM lesions, multifocal infarction and formation of syncytial knots will produce and secrete antiangiogenic protein soluble fms-like tyrosine kinase (sFlt-1) and suppress the secretion of placental growth factor (PlGF) in the maternal blood [56-58]. A prospective cohort study linking longitudinal changes in maternal angiogenic biomarkers and uterine artery doppler measurement (as a proxy to spiral artery adaptation) to postpartum pathological placental lesions of MVM would provide more insight into the intricate mechanism of placental aetiology of FGR. The use of placental biomarkers in predictive models for FGR can have major implications on our future screening strategies. Although our model of combining first trimester placental biomarkers and third trimester fetal growth velocities significantly improved the prediction of pregnancies with MVM lesions, the applicability of this model is hampered by the relatively low positive and negative predictive value of about $50 \%$. Interestingly, the addition of these biomarkers, together with measuring fetal growth velocity, have been shown to improve the clinical screening strategies and identification of high-risk pregnancies, and allow timely interventions and targeted therapies [11, 59].

\section{Strengths and limitations}

There are certain limitations in the current study that need to be highlighted. First of all, the study has a retrospective design that puts it at risk of selection bias, a part of the cases were not eligible for selection if the placental examination report was not completed. Another limitation is the fact that the study is performed in a tertiary care hospital, confiding the selection to a relatively high-risk population and limiting the interpretation of our data and the generalizability to an unselected low-risk population.

Strengths of the study included the complete integrated registration of various ultrasound, histological and biochemical parameters, as well as maternal and neonatal outcomes from a large population followed up in a single tertiary care centre. The inclusion of ultrasound measurement of fetal growth velocities is a new and practical method of assessing fetal growth potential within the SGA and AGA group using a simple calculation that can be readily employed and interpreted in low-resource setting without the need of complex mathematical models. Another strength of this study is the availability of an appropriate reference group without preeclampsia, hypertensive disease or diabetes. Also, we used the Amsterdam Placental Workshop Consensus Criteria to classify MVM lesions, this standardized definition will help ensure validity and comparability of findings across different studies [27]. 


\section{CONCLUSION}

This study shows that, in neonates considered appropriate for gestational age, there was a negative correlation between their birthweight and placental vascular malperfusion lesions and also adverse neonatal outcome. This suggests that a subgroup of normal weight neonates have features of intrauterine growth restriction and are potentially at risk of neonatal complications due to masked placental dysfunction. Further prospective studies are needed to determine if morbidity in AGA neonates could be reduced by screening using a combination of placental biomarkers and fetal growth velocity. 


\section{REFERENCES}

1. Figueras F, Gratacos E. Update on the diagnosis and classification of fetal growth restriction and proposal of a stage-based management protocol. Fetal Diagn Ther. 2014;36(2):8698.

2. Figueras F, Gardosi J. Intrauterine growth restriction: new concepts in antenatal surveillance, diagnosis, and management. Am J Obstet Gynecol. 2011;204(4):288-300.

3. Lees C, Marlow N, Arabin B, Bilardo CM, Brezinka C, Derks JB, et al. Perinatal morbidity and mortality in early-onset fetal growth restriction: cohort outcomes of the trial of randomized umbilical and fetal flow in Europe (TRUFFLE). Ultrasound Obstet Gynecol. 2013;42(4):400-8.

4. S MK, Gardosi J. Perinatal mortality and fetal growth restriction. Best Pract Res Clin Obstet Gynaecol. 2004;18(3):397-410.

5. Pallotto EK, Kilbride HW. Perinatal outcome and later implications of intrauterine growth restriction. Clin Obstet Gynecol. 2006;49(2):257-69.

6. Kovo M, Schreiber L, Ben-Haroush A, Cohen G, Weiner E, Golan A, et al. The placental factor in early- and late-onset normotensive fetal growth restriction. Placenta. 2013;34(4):320-4.

7. Papageorghiou AT, Ohuma EO, Altman DG, Todros T, Cheikh Ismail L, Lambert A, et al. International standards for fetal growth based on serial ultrasound measurements: the Fetal Growth Longitudinal Study of the INTERGROWTH-21st Project. Lancet. 2014;384(9946):869-79.

8. Chauhan SP, Magann EF. Screening for fetal growth restriction. Clin Obstet Gynecol. 2006;49(2):284-94.

9. Gardosi J, Madurasinghe V, Williams M, Malik A, Francis A. Maternal and fetal risk factors for stillbirth: population based study. BMJ. 2013;346:f108.

10. Sovio U, White IR, Dacey A, Pasupathy D, Smith GCS. Screening for fetal growth restriction with universal third trimester ultrasonography in nulliparous women in the Pregnancy Outcome Prediction (POP) study: a prospective cohort study. Lancet. 2015;386(10008):208997.

11. MacDonald TM, Hui L, Tong S, Robinson AJ, Dane KM, Middleton AL, et al. Reduced growth velocity across the third trimester is associated with placental insufficiency in fetuses born at a normal birthweight: a prospective cohort study. BMC Med. 2017;15(1):164.

12. Vrachnis N, Kalampokas E, Sifakis S, Vitoratos N, Kalampokas T, Botsis D, et al. Placental growth factor (PIGF): a key to optimizing fetal growth. J Matern Fetal Neonatal Med. 2013;26(10):995-1002.

13. Torry DS, Hinrichs M, Torry RJ. Determinants of placental vascularity. Am J Reprod Immunol. 2004;51(4):257-68.

14. Tong M, Kleffmann T, Pradhan S, Johansson CL, DeSousa J, Stone PR, et al. Proteomic characterization of macro-, micro- and nano-extracellular vesicles derived from the same first trimester placenta: relevance for feto-maternal communication. Hum Reprod. 2016;31(4):687-99.

15. Powe CE, Levine RJ, Karumanchi SA. Preeclampsia, a disease of the maternal endothelium: the role of antiangiogenic factors and implications for later cardiovascular disease. Circulation. 2011;123(24):2856-69. 
16. Binder NK, Evans J, Salamonsen LA, Gardner DK, Kaitu'u-Lino TJ, Hannan NJ. Placental Growth Factor Is Secreted by the Human Endometrium and Has Potential Important Functions during Embryo Development and Implantation. PLoS One. 2016;11(10):e0163096.

17. Rana S, Karumanchi SA, Levine RJ, Venkatesha S, Rauh-Hain JA, Tamez H, et al. Sequential changes in antiangiogenic factors in early pregnancy and risk of developing preeclampsia. Hypertension. 2007;50(1):137-42.

18. Erez O, Romero R, Espinoza J, Fu W, Todem D, Kusanovic JP, et al. The change in concentrations of angiogenic and anti-angiogenic factors in maternal plasma between the first and second trimesters in risk assessment for the subsequent development of preeclampsia and small-for-gestational age. J Matern Fetal Neonatal Med. 2008;21(5):279-87.

19. Taylor RN, Grimwood J, Taylor RS, McMaster MT, Fisher SJ, North RA. Longitudinal serum concentrations of placental growth factor: evidence for abnormal placental angiogenesis in pathologic pregnancies. Am J Obstet Gynecol. 2003;188(1):177-82.

20. Crispi F, Dominguez C, Llurba E, Martin-Gallan P, Cabero L, Gratacos E. Placental angiogenic growth factors and uterine artery Doppler findings for characterization of different subsets in preeclampsia and in isolated intrauterine growth restriction. Am J Obstet Gynecol. 2006;195(1):201-7.

21. Romero R, Nien JK, Espinoza J, Todem D, Fu W, Chung H, et al. A longitudinal study of angiogenic (placental growth factor) and anti-angiogenic (soluble endoglin and soluble vascular endothelial growth factor receptor-1) factors in normal pregnancy and patients destined to develop preeclampsia and deliver a small for gestational age neonate. J Matern Fetal Neonatal Med. 2008;21(1):9-23.

22. Karumanchi SA, Epstein FH. Placental ischemia and soluble fms-like tyrosine kinase 1: cause or consequence of preeclampsia? Kidney Int. 2007;71(10):959-61.

23. Wright E, Audette MC, Ye XY, Keating S, Hoffman B, Lye SJ, et al. Maternal Vascular Malperfusion and Adverse Perinatal Outcomes in Low-Risk Nulliparous Women. Obstet Gynecol. 2017;130(5):1112-20.

24. Gluck O, Schreiber L, Marciano A, Mizrachi Y, Bar J, Kovo M. Pregnancy outcome and placental pathology in small for gestational age neonates in relation to the severity of their growth restriction. J Matern Fetal Neonatal Med. 2017:1-6.

25. Voskamp BJ, Kazemier BM, Ravelli AC, Schaaf J, Mol BW, Pajkrt E. Recurrence of small-forgestational-age pregnancy: analysis of first and subsequent singleton pregnancies in The Netherlands. Am J Obstet Gynecol. 2013;208(5):374 e1-6.

26. Levy M, Kovo M, Schreiber L, Kleiner I, Koren L, Barda G, et al. Pregnancy outcomes in correlation with placental histopathology in subsequent pregnancies complicated by preeclampsia. Pregnancy Hypertens. 2019;18:163-8.

27. Khong TY, Mooney EE, Ariel I, Balmus NC, Boyd TK, Brundler MA, et al. Sampling and Definitions of Placental Lesions: Amsterdam Placental Workshop Group Consensus Statement. Arch Pathol Lab Med. 2016;140(7):698-713.

28. Brown MA, Lindheimer MD, de Swiet M, Van Assche A, Moutquin JM. The classification and diagnosis of the hypertensive disorders of pregnancy: statement from the International Society for the Study of Hypertension in Pregnancy (ISSHP). Hypertens Pregnancy. 2001;20(1):IX-XIV.

29. Kloosterman GJ. [Intrauterine growth and intrauterine growth curves]. Ned Tijdschr Verloskd Gynaecol. 1969;69(5):349-65. 
30. Practice guidelines for performance of the routine mid-trimester fetal ultrasound scan. [Internet]. 2010.

31. Caradeux J, Eixarch E, Mazarico E, Basuki TR, Gratacos E, Figueras F. Second to third trimester longitudinal growth assessment for the prediction of SGA and late FGR. Ultrasound Obstet Gynecol. 2017.

32. Hadlock FP, Harrist RB, Sharman RS, Deter RL, Park SK. Estimation of fetal weight with the use of head, body, and femur measurements-a prospective study. Am J Obstet Gynecol. 1985;151(3):333-7.

33. Hendrix MLE, van Kuijk SMJ, Gavilanes AWD, Kramer D, Spaanderman MEA, Al Nasiry S. Reduced fetal growth velocities and the association with neonatal outcomes in appropriate-for-gestational-age neonates: a retrospective cohort study. BMC Pregnancy Childbirth. 2019;19(1):31.

34. Mathis G. Rare earth cryptates and homogeneous fluoroimmunoassays with human sera. Clin Chem. 1993;39(9):1953-9.

35. Sweet DG, Carnielli V, Greisen G, Hallman M, Ozek E, Plavka R, et al. European Consensus Guidelines on the Management of Respiratory Distress Syndrome - 2016 Update. Neonatology. 2017;111(2):107-25.

36. Buchiboyina A, Jasani B, Deshmukh M, Patole S. Strategies for managing transient tachypnoea of the newborn - a systematic review. J Matern Fetal Neonatal Med. 2017;30(13):152432.

37. Morales P, Bustamante D, Espina-Marchant P, Neira-Pena T, Gutierrez-Hernandez MA, Allende-Castro C, et al. Pathophysiology of perinatal asphyxia: can we predict and improve individual outcomes? Epma j. 2011;2(2):211-30.

38. Kraus FT RR, Gersell DJ, Nelson DM, Dicke JM. Placental pathology. Atlas of Nontumor Pathology2004. p. 75-115.

39. Burton GJ, Jauniaux E. Pathophysiology of placental-derived fetal growth restriction. Am J Obstet Gynecol. 2018;218(2S):S745-S61.

40. Chisholm KM, Folkins AK. Placental and Clinical Characteristics of Term Small-forGestational-Age Neonates: A Case-Control Study. Pediatr Dev Pathol. 2016;19(1):37-46.

41. Parra-Saavedra M, Crovetto F, Triunfo S, Savchev S, Peguero A, Nadal A, et al. Placental findings in late-onset SGA births without Doppler signs of placental insufficiency. Placenta. 2013;34(12):1136-41.

42. Parra-Saavedra M, Simeone S, Triunfo S, Crovetto F, Botet F, Nadal A, et al. Correlation between histological signs of placental underperfusion and perinatal morbidity in late-onset small-for-gestational-age fetuses. Ultrasound in obstetrics \& gynecology : the official journal of the International Society of Ultrasound in Obstetrics and Gynecology. 2015;45(2):149-55.

43. McIntire DD, Bloom SL, Casey BM, Leveno KJ. Birth weight in relation to morbidity and mortality among newborn infants. N Engl J Med. 1999;340(16):1234-8.

44. Gordijn SJ, Beune IM, Thilaganathan B, Papageorghiou A, Baschat AA, Baker PN, et al. Consensus definition of fetal growth restriction: a Delphi procedure. Ultrasound Obstet Gynecol. 2016;48(3):333-9.

45. Beune IM, Bloomfield FH, Ganzevoort W, Embleton ND, Rozance PJ, van WassenaerLeemhuis AG, et al. Consensus Based Definition of Growth Restriction in the Newborn. J Pediatr. 2018;196:71-6 e1. 
46. Ganzevoort W. Fetal growth and risk assessment: is there an impasse? AJOG. 2019;220:7482.

47. Hendrix MLE, Bons JAP, Snellings RRG, Bekers O, van Kuijk SMJ, Spaanderman MEA, et al. Can Fetal Growth Velocity and First Trimester Maternal Biomarkers Improve the Prediction of Small-for-Gestational Age and Adverse Neonatal Outcome? Fetal Diagn Ther. 2019;46(4):274-84.

48. Figueras F, Caradeux J, Crispi F, Eixarch E, Peguero A, Gratacos E. Diagnosis and surveillance of late-onset fetal growth restriction. Am J Obstet Gynecol. 2018;218(2S):S790-S802 e1.

49. Boers KE, Vijgen SM, Bijlenga D, van der Post JA, Bekedam DJ, Kwee A, et al. Induction versus expectant monitoring for intrauterine growth restriction at term: randomised equivalence trial (DIGITAT). BMJ. 2010;341:c7087.

50. Bukowski R, Hansen NI, Pinar H, Willinger M, Reddy UM, Parker CB, et al. Altered fetal growth, placental abnormalities, and stillbirth. PLoS One. 2017;12(8):e0182874.

51. Nardozza LM, Caetano AC, Zamarian AC, Mazzola JB, Silva CP, Marcal VM, et al. Fetal growth restriction: current knowledge. Arch Gynecol Obstet. 2017;295(5):1061-77.

52. Bustamante Helfrich B, Chilukuri N, He H, Cerda SR, Hong X, Wang G, et al. Maternal vascular malperfusion of the placental bed associated with hypertensive disorders in the Boston Birth Cohort. Placenta. 2017;52:106-13.

53. Schoots MH, Gordijn SJ, Scherjon SA, van Goor H, Hillebrands JL. Oxidative stress in placental pathology. Placenta. 2018;69:153-61.

54. Hung TH, Skepper JN, Burton GJ. In vitro ischemia-reperfusion injury in term human placenta as a model for oxidative stress in pathological pregnancies. Am J Pathol. 2001;159(3):1031-43.

55. Morgan TK, Tolosa JE, Mele L, Wapner RJ, Spong CY, Sorokin Y, et al. Placental villous hypermaturation is associated with idiopathic preterm birth. J Matern Fetal Neonatal Med. 2013;26(7):647-53.

56. Johansen M, Redman CW, Wilkins T, Sargent IL. Trophoblast deportation in human pregnancy-its relevance for pre-eclampsia. Placenta. 1999;20(7):531-9.

57. Sekizawa A, Purwosunu Y, Farina A, Shimizu H, Nakamura M, Wibowo N, et al. Prediction of pre-eclampsia by an analysis of placenta-derived cellular mRNA in the blood of pregnant women at 15-20 weeks of gestation. BJOG. 2010;117(5):557-64.

58. Widmer M, Cuesta C, Khan KS, Conde-Agudelo A, Carroli G, Fusey S, et al. Accuracy of angiogenic biomarkers at 20weeks' gestation in predicting the risk of pre-eclampsia: A WHO multicentre study. Pregnancy Hypertens. 2015;5(4):330-8.

59. Bardien N, Whitehead CL, Tong S, Ugoni A, McDonald S, Walker SP. Placental Insufficiency in Fetuses That Slow in Growth but Are Born Appropriate for Gestational Age: A Prospective Longitudinal Study. PLoS One. 2016;11(1):e0142788. 


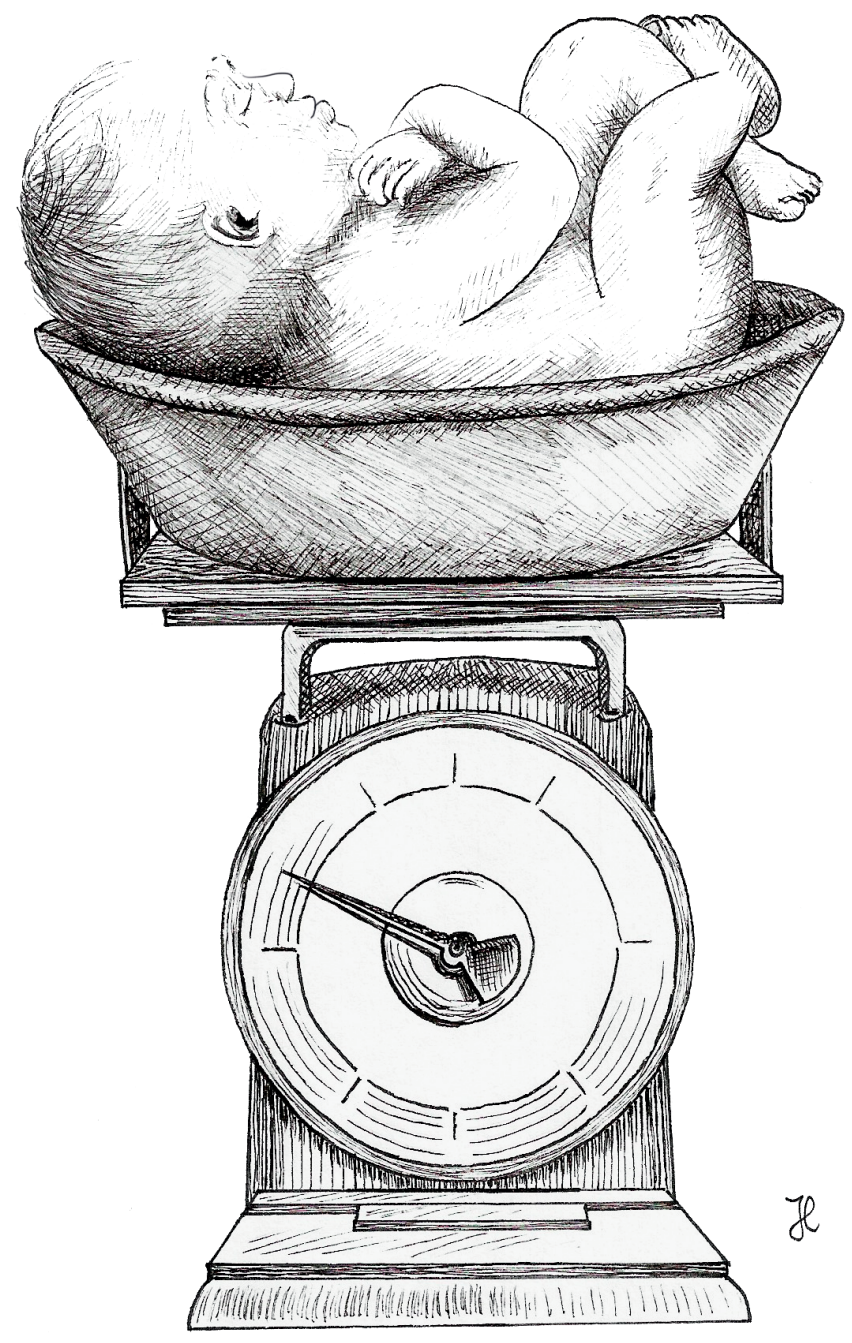




\section{First trimester prediction of term SGA and reduced fetal growth velocity is poor.}

MLE Hendrix

WPTM van Doorn

BAM Conrads

HCJ Scheepers

BW Kramer

MEA Spaanderman

JAP Bons

S Al-Nasiry

Submitted

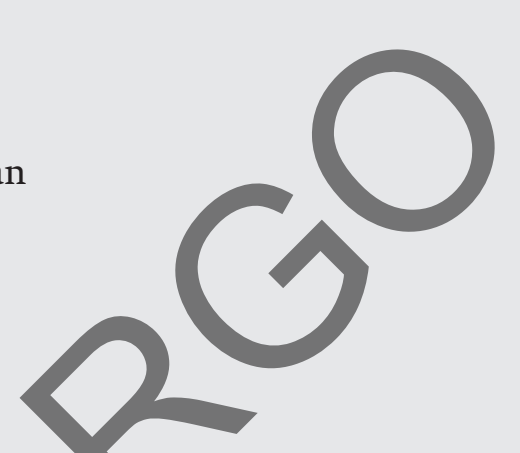


Postnatal growth during the first five years of life in SGA and AGA neonates with reduced fetal growth

\author{
MLE Hendrix \\ SMJ van Kuijk \\ SE El Bahaey \\ WJM Gerver \\ FJM Feron \\ ML Kuin \\ MEA Spaanderman \\ JAP Bons \\ S Al-Nasiry
}

Early Human Development. 2020 Sep 23;151:105199. 


\section{ABSTRACT}

\section{Background}

Even though a lot of research has been done on postnatal growth and the occurrence of catch-up growth in small-for-gestational age (SGA) neonates, this phenomenon has not been studied well in appropriate-for-gestational-age (AGA) neonates. Postnatal catch-up growth may also occur in AGA neonates indicating a compensatory mechanism for undiagnosed intrauterine growth restriction, especially in AGA neonates with reduced fetal growth velocity.

\section{Aims}

To describe postnatal growth during the first 5 years of life in SGA and AGA neonates and evaluating the role of fetal growth velocity in catch-up growth.

\section{Study design}

Retrospective study in a Dutch tertiary hospital.

\section{Subjects}

740 singleton neonates, without congenital anomalies, with ultrasound fetal growth data from 20 weeks and 32 weeks of pregnancy.

\section{Outcome measures}

Postnatal growth measurements of height $(\mathrm{cm})$ and weight $(\mathrm{kg})$ from birth until five years of age.

Postnatal catch-up growth defined as difference (delta) in both height and weight between 4 weeks and 3 years of age.

\section{Results and conclusion}

SGA neonates had a significantly lower height and weight compared to the AGA group for all available measurement moments till 3 years. The catch-up growth between the SGA and AGA groups from 4 weeks up to 3 years after birth was not different between the two groups. However, neonates with reduced fetal growth velocity had a significantly higher risk for catch-up growth in height during the first 3 years after birth.

This suggests a role for fetal growth velocity measurement in predicting fetal and subsequent postnatal growth potential.

\section{Keywords}

Birthweight percentile, Fetal growth velocity, IUGR, FGR, catch-up growth 


\section{INTRODUCTION}

Fetal growth during pregnancy is a complex biological process determined by a multitude of intrinsic (epi)genetic factors, maternal, constitutional and environmental factors, and placental function [1]. Fetal growth restriction (FGR) is defined as intra uterine growth less than the growth potential [2] and has been associated with neonatal morbidity and mortality $[3,4,5]$. Both adequate fetal growth and postnatal growth are of crucial importance for any infant during the first years of life. Some of the long-term health impacts of FGR are increased risk of developing cardiovascular disease, obesity and metabolic syndrome [6].

Neonatal birth weight has long been considered as a surrogate for fetal growth. Nonetheless, as antenatal and postnatal growth charts are separately adapted, a fetus with FGR during pregnancy can be born with either a reduced or normal birthweight, and hence classified either as small-for-gestational age (SGA) or appropriate-for-gestational-age (AGA) [7]. SGA is often defined as a birth weight below the tenth percentile of a certain reference at a given gestational age or as a birth weight being less than two standard deviations below the population mean. Although SGA is often considered fetal growth restriction, this is not necessarily the case [8]. Most SGA infants are constitutionally small but healthy, without impaired growth potential [9]. The usual postnatal growth pattern of SGA infants is an increased growth velocity in the first 2-3 years, followed by a stable height development in further childhood. This acceleration in growth after a period of growth inhibition and characterized by a growth velocity above the statistical limits of normal for age for at least one year is called catch-up growth [10]. Most infants born premature and classified as SGA have a slower and more prolonged catch-up growth period than term SGA infants, and it can take 4 or more years for the premature infants to fully catch-up [10 -13]. Infants who are born as SGA and did not undergo postnatal catch-up growth for height till the age of two had a greater risk for developing short stature at adulthood. The risk for short stature at adulthood is 7 times higher for neonates with a lower birth height as opposed to only 5 times for neonates who had a lower birthweight [14]. This highlights the importance for height as a parameter in determining catch-up growth.

The mechanisms that lead to persistence of short stature in infants, who meet the SGA criteria at birth, are not yet well understood $[15,16]$. Even though a lot of research has been done on postnatal growth and the occurrence of catch-up growth in SGA infants, this phenomenon has not been studied well in term AGA infants.

In preterm AGA infants are comorbidities (respiratory distress syndrome, sepsis and patent ductus arteriosus) associated with postnatal growth failure [17] . 
However, in literature there is still a gap on information about postnatal growth in term AGA neonates. Postnatal catch-up growth may also occur in AGA infants indicating a compensatory mechanism for undiagnosed intrauterine growth restriction, especially in infants with reduced fetal growth velocity. In this line of reasoning, catch-up growth is probably more linked to fetal growth velocity, irrespective to birth weight [18]. In AGA infants, this compensatory catch-up growth often goes unnoticed, as these infants are considered completely healthy, based on birth weight, leading to an underestimation and late recognition of postnatal catch-up growth in this particular group of infants [19-21] We hypothesized that postnatal growth trajectories differ between SGA and AGA neonates, and are affected by fetal growth velocity between 20-32 weeks of pregnancy.

\section{MATERIAL AND METHODS}

\section{Study design and participants}

In this retrospective cohort study, we studied 740 singleton neonates, without congenital anomalies, born in a Dutch tertiary hospital between January 2011 and June 2016. We combined data from two electronic patient databases: the antenatal ultrasound database of the Maastricht University Medical Center (MUMC+) and the growth measurements database of the Child Health Care Services ZuidLimburg, Limburg-Noord and Hart voor Brabant to make an integrated database.

Offspring was only included in this cohort if they had two available fetal growth scans, one between 18 and 22 weeks of gestational age and one between 30 and 34 weeks of gestational age. The pregnancies were dated according to the formula of Robinson calculated from fetal Crown-Rump Length (CRL) measurement in the first trimester [22]. Exclusion criteria were neonates with no available growth measurements of height, weight and head circumference after birth.

Delivery and neonatal outcomes were registered by the obstetrician or in case of an adverse outcome by the paediatrician. APGAR-scores were assessed at 1 and 5 minutes after birth. Metabolic acidosis was defined as an umbilical artery blood $\mathrm{pH}<7.0$ and base deficit $>12 \mathrm{mmol} / \mathrm{L}$. Hospital stay and neonatal intensive care unit stay was expressed in days. Patients were informed about research in the MUMC+, and were given information about the data collection from their records for research purpose. Patients who refused using their records, were excluded in this study. The study protocol was approved by the medical ethical committee of the Maastricht University Medical Centre (17-4-0.15.1/ab). All procedures were in accordance with institutional guidelines and adhered to the principles of the Declaration of Helsinki and Title 45, U.S. Code of Federal Regulations, Part 46, 
Protection of Human Subjects (revised 13 November 2001, effective 13 December 2001).

\section{Ultrasound growth examination and growth measurements}

Maternal data were collected at the first hospital intake. Maternal age, data on height, weight, smoking status and medical and obstetric history were recorded in an electronic medical file. Body mass index (BMI) was calculated by weight (kg) divided by height (m)-squared.

Ultrasound measurements of abdominal circumference (AC) were recorded by an experienced sonographer during routine prenatal care visits at the MUMC+, using a GE Voluson ultrasound machine, with a $2-5 \mathrm{MHz}$ curved-array transducer, in accordance with the routine mid-trimester fetal ultrasound scan guidelines [23]. These fetal growth scans were performed in two periods: one around 20 weeks (18-22 weeks) of gestational age during the second trimester anomaly scan and a second around 32 weeks (30-34 weeks) as a routine third trimester growth scan [24, 25]. Fetal growth parameters were recorded in Astraia electronic fetal medicine database (Astraia GMBH; Munich, Germany). Estimated fetal weight was calculated using the Hadlock equation [Hadlock C; $\log (10) \mathrm{BW}=1.335-0.0034(\mathrm{AC})$ $(\mathrm{FL})+0.0316(\mathrm{BPD})+0.0457(\mathrm{AC})+0.1623(\mathrm{FL})[26]$. In line with our previously published articles, we calculated fetal abdominal circumference growth velocity (ACv) as the difference in absolute value of the measured abdominal circumference between the two examination periods, divided by the number of weeks (mm/ week) [27]. In addition, fetal growth velocity was also expressed as a dichotomous outcome, and consequently fetal reduced growth velocity was defined as an AC growth velocity between 20 and 32 weeks in the lowest quartile $(<25 \%)[7,28]$. This pragmatic choice of the lowest quartile of AC growth velocity to define reduced fetal growth is based on previous reports showing that fetal growth between the $25^{\text {th }}-75^{\text {th }}$ percentile could be protective for stillbirths $[29,30]$.

Neonatal birth weight was measured shortly after birth. Birth weight was transformed to percentiles according to the Dutch reference standard, with a birth weight $>10^{\text {th }}$ percentile considered to be AGA and a birth weight $<10^{\text {th }}$ percentile considered as SGA. Postnatal growth measurements in height $(\mathrm{cm})$ and weight (kg) were collected from birth until five years of age. Head circumference $(\mathrm{cm})$ was measured up to 6 months after birth. The parameters were measured at the Community Health Service during standard regular appointments (at 1, 2, 3, 4, 6, 12 and 18 months after birth and 2, 3 and 5 years after birth).

Until the age of 18 months, the neonate height was measured in supine position, using a measuring tray consisting of a ground board, headboard and movable feetboard. Thereafter height was measured in standing position, using a stadiom- 
eter. The scale was accurate to 1 millimeter. In the first year of life weight was measured using an electronic scale (accuracy of 10 grams). After the first year of life, an accuracy of 100 grams was sufficient. Head circumference was measured with a non-stretchable tape around the most protruding points of occiput and forehead [31, 32]. All measurements were recorded by an experienced assistant. Catch-up growth is traditionally observed during the first year and continues up to 3 years of age. In this study catch-up growth was calculated as the increase in a growth parameter (e.g. height and weight) between 4 weeks and 3 years [33].

\section{Statistical analysis}

Baseline characteristics of the study sample were quantified as means with standard deviations (SD), median with range or percentage with count. Comparisons between the groups were made using the independent samples t- test or the Mann-Whitney U test for continuous data. For comparing categorical data between groups, the $\chi^{2}$ test was performed. Differences in means of growth measurements between the four groups were tested using one-way analysis of variance (ANOVA). Bonferroni post hoc test was applied to compare each two groups.

For the longitudinal analyses, we first selected the best fitting model of growth in height and weight over time by consecutively fitting the Count and first- and second-order Reed growth models [34]. In addition to age in years, the Count model includes a term for the natural logarithm of age. The first- and second order Reed models both include an additional term for the reciprocal of age, and the second order also include the reciprocal squared. The models are given below as 1 (Count), 2 (first-order Reed) and 3 (second-order Reed):

[1] $\mathrm{Y}=\mathrm{a}+\mathrm{bX}+\mathrm{c}(\ln \mathrm{X})$

[2] $\mathrm{Y}=\mathrm{a}+\mathrm{bX}+\mathrm{c}(\ln \mathrm{X})+\mathrm{d}(1 / \mathrm{X})$

[3] $\mathrm{Y}=\mathrm{a}+\mathrm{bX}+\mathrm{c}(\ln \mathrm{X})+\mathrm{d}(1 / \mathrm{X})+\mathrm{e}(1 / \mathrm{X})^{2}$

In which $\mathrm{X}$ denotes age in years, and $\mathrm{ln}$ is the natural logarithm.

All models were fitting using a linear mixed-effects model with random intercept and random slope for all covariates of the growth function. We used an unstructured variance-covariance matrix for the random effects. The best fitting model was selected using the Akaike Information Criterion (AIC). The model with the lowest AIC was chosen, as this reflects a better fit given the number of parameters in the model.

Next, we added an indicator variable for SGA to the model, and an interaction between the indicator and growth model parameters to evaluate whether there are differences in growth between SGA and AGA neonates. All analyses were 
performed using $\mathrm{R}$ version 3.5.1. For comparing SGA and AGA neonates, a P-value of $<0.05$ was considered to indicate statistical significance.

\section{RESULTS}

\section{Maternal and neonatal characteristics}

The baseline maternal and neonatal characteristics of the total study cohort $(\mathrm{n}=740)$, and divided in the AGA $(\mathrm{P} 10-90 ; \mathrm{n}=690)$ as reference group and SGA $(\mathrm{p}<10 ; \mathrm{n}=50)$ group are shown in Table 1 . Maternal height was significantly lower in the SGA than in the AGA group ( $\mathrm{p}<.002$ ). The pregnancy duration was also significantly shorter in SGA neonates $(\mathrm{p}=0.004)$ and there were more preterm births in the SGA group compared with AGA $(\mathrm{P}<.0001)$. SGA neonates stayed longer in the hospital compared with AGA neonates $(\mathrm{p}=0.005)$. The other observed parameters did not significantly differ between both groups.

\section{Postnatal parameters (height, weight and head circumference)}

There were significant differences between SGA and AGA neonates in the observed postnatal growth parameters from 4 weeks up to 5 years (Table 2). The SGA group had significantly lower height and weight compared to the AGA group for all measurements till 3 years. Even at the age of 5 years, there was a significant difference in height (113.6 vs $120.0 \mathrm{~cm}, \mathrm{p}=0.047)$, but there was no statistical significant difference in weight between the AGA and SGA group (23.5 vs $20.0 \mathrm{~kg}$, $\mathrm{p}=0.069$ ). The head circumference was significantly lower until 6 months in the SGA group compared with AGA (42.6 vs $43.5 \mathrm{~cm}, \mathrm{p}=0.036$ ).

There was a mild but significant correlation between the AC velocity at the 20-32 weeks interval of pregnancy and neonatal weight in the first five years after birth $(\mathrm{r}=0.379, \mathrm{p}=0.019)$ and neonatal height till 3 years after birth $(\mathrm{r}=0.182, \mathrm{p}=0.027)$.

The AGA and SGA group were further stratified based on the AC velocity between 20 and 32 weeks, into those with a reduced $A C$ velocity ( $\mathrm{ACv}<25 \%)$ and those with a normal AC velocity (ACv $>25 \%$ ) (Table 3 ). Both SGA groups (with reduced and normal ACv) had significantly lower height measurements till 3 years compared to the reference group (AGA, ACv $>25 \%$ ), but a higher height measurement at 5 years of age $(\mathrm{p}=0.045, \mathrm{p}=0.041)$. Interestingly, also AGA neonates with reduced $\mathrm{ACv}$ showed lower height measurements compared to the reference group (AGA, $\mathrm{ACv}>25 \%$ ) till 18 months ( $\mathrm{p}=0.036)$.

Similarly, regarding postnatal weight measurements, both SGA groups had significantly lower weight measurements compared to the reference group (AGA, $\mathrm{ACv}>25 \%$ ) at all time intervals. Again, AGA neonates with reduced ACv showed 
lower weight measurements compared to the reference group (AGA, ACv >25\%) from 8 weeks to 3 years.

Our research demonstrated that SGA with reduced ACv had lower height and weight measurements compared to SGA with normal AC velocity till 12 months, and a reversed trend for higher height and weight measurements after 12 months

Table 1. Baseline characteristics of the total study cohort, AGA (reference group) and SGA group.

\begin{tabular}{|c|c|c|c|c|}
\hline Characteristic & $\begin{array}{l}\text { Total cohort study } \\
\qquad \mathrm{n}=740\end{array}$ & $\begin{array}{l}\text { AGA group (control } \\
\text { group) } n=690\end{array}$ & $\begin{array}{l}\text { SGA group } \\
\quad n=50\end{array}$ & P-value \\
\hline Age (years) & $31.6 \pm 4.9(18-55)$ & $31.7 \pm 4.8(18-55)$ & $30.5 \pm 4.2(23-40)$ & 0.115 \\
\hline BMI $\left(\mathrm{kg} / \mathrm{m}^{2}\right)$ & $25.3 \pm 5.7(16.5-49.0)$ & $25.4 \pm 5.7(16.5-49.0)$ & $23.8 \pm 50(18.0-42.8)$ & 0.075 \\
\hline Height $(\mathrm{cm})$ & $167.3 \pm 6.7(146-188)$ & $167.5 \pm 6.7(146-188)$ & $164.1 \pm 6.2(150-179)$ & 0.002 \\
\hline $\begin{array}{l}\text { Pregnancy duration } \\
\text { (days) }\end{array}$ & $271.5 \pm 12.4(212-295)$ & $272.0 \pm 11.8(212-292)$ & $\begin{array}{c}264.4 \pm 17.5(224- \\
290)\end{array}$ & 0.004 \\
\hline Preterm birth & $69(9.3 \%)$ & $52(7.5 \%)$ & 17 (34\%) & $<.0001$ \\
\hline Primigravida & $270(36.6 \%)$ & $231(36.4 \%)$ & 38 (76.0\%) & $<0.001$ \\
\hline $\begin{array}{l}\text { Smoking during } \\
\text { pregnancy }\end{array}$ & & & & 0.519 \\
\hline Yes & $66(8.9 \%)$ & $60(8.7 \%)$ & $6(12.0 \%)$ & \\
\hline No & 535 (72.3\%) & $498(89.2 \%)$ & $37(74.0 \%)$ & \\
\hline Missing & $139(18.8 \%)$ & $132(19.1 \%)$ & $7(14 \%)$ & \\
\hline Birth weight (gram) & $\begin{array}{c}3342.5 \pm 542.5 \\
(1353-4860)\end{array}$ & $\begin{array}{c}3402.4 \pm 496.4 \\
(1573-4860)\end{array}$ & $\begin{array}{c}2516.5 \pm 478.1 \\
(1353-3255)\end{array}$ & $<.0001$ \\
\hline Gender child & & & & 0.194 \\
\hline Male & $377(50,9 \%)$ & $356(51,6 \%)$ & $21(42,0 \%)$ & \\
\hline Female & $363(49,1 \%)$ & $344(48,4 \%)$ & $29(58,0 \%)$ & \\
\hline Apgar 1 & $8 \pm 1(1-10)$ & $8 \pm 1(1-10)$ & $8 \pm 1(4-10)$ & 0.775 \\
\hline Apgar 5 & $9 \pm 1(3-10)$ & $9 \pm 1(3-10)$ & $9 \pm 1(6-10)$ & 0.500 \\
\hline $\mathrm{pH}$ arterial & $7.2 \pm 0.1(6.9-7.5)$ & $7.2 \pm 0.1(6.9-7.4)$ & $7.2 \pm 0.1(7.1-7.5)$ & 0.572 \\
\hline $\mathrm{pH}$ venous & $7.3 \pm 0.1(7.0-7.5)$ & $7.3 \pm 0,1(7.0-7.5)$ & $7.3 \pm 0.1(7.2-7.5)$ & 0.928 \\
\hline $\mathrm{BE}$ arterial & $-6.3 \pm 3.6(-20.0-4.1)$ & $-6.2 \pm 3.5(-20.0-4.1)$ & $-7.0 \pm 4.0(-16.5-2.2)$ & 0.217 \\
\hline $\mathrm{BE}$ venous & $-5.6 \pm 2.9(-17.0-4.6)$ & $-5.6 \pm 2.9(-17.0-4.6)$ & $-6.4 \pm 3.1(-11.9-3.8)$ & 0.098 \\
\hline Metabolic acidosis & & & & 0.597 \\
\hline Yes & $40(5.4 \%)$ & 38 (5.5\%) & $2(4.0 \%)$ & \\
\hline No & 540 (73.0\%) & $501(72.6 \%)$ & 39 (78.0\%) & \\
\hline Missing & $160(21.6 \%)$ & $151(21.9 \%)$ & $9(18 \%)$ & \\
\hline Hospital stay (yes) & $402(54.3 \%)$ & 374 (54.2\%) & $28(56.0 \%)$ & 0.806 \\
\hline Hospital stay (days) & $3.9 \pm 5.4(0-50)$ & $3.7 \pm 4.9(0-50)$ & $7.8 \pm 9.8(0-41)$ & 0.005 \\
\hline NICU stay (yes) & $54(7.3 \%)$ & $47(6.8 \%)$ & 7 (14.0\%) & 0.160 \\
\hline NICU stay (days) & $0.44 \pm 2.58(0-50)$ & $0.4 \pm 2.5(0-50)$ & $1.3 \pm 3.5(0-14)$ & 0.081 \\
\hline
\end{tabular}

Data presented as mean \pm standard deviation and (minimum - maximum) or as N (\%). BMI, Body Mass Index; SGA, small-for-gestational age; AGA, appropriate-for-gestational age; BE, base excess; NICU, Neonatal Intensive Care Unit. 
(Table 3). For postnatal head circumference, only the group of SGA neonates with reduced $\mathrm{ACv}$ had lower measurements compared to the reference group (AGA with normal $\mathrm{ACv}$ ) at all time points.

Table 2. Mean value of postnatal height $(\mathrm{cm})$, weight $(\mathrm{kg})$ and head circumference $(\mathrm{cm})$ measured at different terms from 4 weeks till 5 years. AGA reference group and SGA study group.

\begin{tabular}{|c|c|c|c|}
\hline & $\begin{array}{c}\text { AGA }(p>10) \\
n=690\end{array}$ & $\begin{array}{c}\text { SGA }(p<10) \\
n=50\end{array}$ & P-value \\
\hline \multicolumn{4}{|l|}{ Height $(\mathrm{cm})$} \\
\hline 4 weeks & $53.8 \pm 2.2$ & $49.7 \pm 2.6$ & $<.0001$ \\
\hline 8 weeks & $57.0 \pm 2.4$ & $53.3 \pm 3.0$ & $<.0001$ \\
\hline 3 months & $60.3 \pm 2.4$ & $56.5 \pm 2.8$ & $<.0001$ \\
\hline 4 months & $63.5 \pm 2.5$ & $59.7 \pm 3.0$ & $<.0001$ \\
\hline 6 months & $67.7 \pm 2.5$ & $64.3 \pm 2.8$ & $<.0001$ \\
\hline 12 months & $75.0 \pm 2.3$ & $72.1 \pm 2.7$ & $<.0001$ \\
\hline 18 months & $82.6 \pm 3.2$ & $79.4 \pm 2.7$ & $<.0001$ \\
\hline 2 years & $88.9 \pm 3.4$ & $86.2 \pm 3.2$ & 0.002 \\
\hline 3 years & $97.5 \pm 3.7$ & $95.1 \pm 4.1$ & 0.018 \\
\hline 5 years & $120.0 \pm 5.2$ & $113.6 \pm 2.5$ & 0.047 \\
\hline \multicolumn{4}{|l|}{ Weight (kg) } \\
\hline 4 weeks & $4.3 \pm 1.5$ & $3.2 \pm 0.5$ & $<.0001$ \\
\hline 8 weeks & $5.2 \pm 0.7$ & $4.0 \pm 0.7$ & $<.0001$ \\
\hline 3 months & $6.0 \pm 0.7$ & $4.8 \pm 0.7$ & $<.0001$ \\
\hline 4 months & $6.8 \pm 0.8$ & $5.6 \pm 0.8$ & $<.0001$ \\
\hline 6 months & $7.9 \pm 0.9$ & $6.6 \pm 0.9$ & $<.0001$ \\
\hline 12 months & $9.8 \pm 1.0$ & $8.5 \pm 0.9$ & $<.0001$ \\
\hline 18 months & $11.5 \pm 1.4$ & $10.0 \pm 1.1$ & $<.0001$ \\
\hline 2 years & $13.1 \pm 1.6$ & $11.6 \pm 1.2$ & 0.002 \\
\hline 3 years & $15.4 \pm 1.9$ & $13.4 \pm 1.6$ & $<.0001$ \\
\hline 5 years & $23.5 \pm 3.2$ & $20.0 \pm 1.6$ & 0.069 \\
\hline \multicolumn{4}{|c|}{ Head circumference $(\mathrm{cm})$} \\
\hline 4 weeks & $37.1 \pm 1.3$ & $36.1 \pm 1.2$ & 0.036 \\
\hline 8 weeks & $38.7 \pm 1.4$ & $38.1 \pm 1.3$ & 0.202 \\
\hline 3 months & $40.2 \pm 1.4$ & $39.4 \pm 1.5$ & 0.048 \\
\hline 4 months & $41.7 \pm 1.4$ & $40.7 \pm 1.7$ & 0.024 \\
\hline 6 months & $43.5 \pm 1.5$ & $42.6 \pm 1.7$ & 0.036 \\
\hline
\end{tabular}

Data presented as mean \pm standard deviation. AGA, appropriate-for-gestational age; SGA, small-for-gestational age. 
Table 3. Mean value of postnatal height $(\mathrm{cm})$, weight $(\mathrm{kg})$ and head circumference $(\mathrm{cm})$ measured at different terms from 4 weeks till 5 years. AGA $+A C v>25 \%$ reference group.

\begin{tabular}{|c|c|c|c|c|c|c|c|}
\hline & $\begin{array}{c}\text { AGA + } \\
\text { ACv }>25 \% \\
\text { (reference } \\
\text { group) } \\
\mathrm{n}=400\end{array}$ & $\begin{array}{c}\mathrm{AGA}+ \\
\mathrm{ACv}<25 \% \\
\mathbf{n}=105\end{array}$ & p-value & $\begin{array}{c}\text { SGA + } \\
\text { ACv }>25 \% \\
n=19\end{array}$ & p-value & $\begin{array}{c}\text { SGA+ } \\
\mathrm{ACv}<25 \% \\
\mathbf{n}=22\end{array}$ & p-value \\
\hline \multicolumn{8}{|l|}{ Height (cm) } \\
\hline 4 weeks & $53.9 \pm 2.1$ & $53.4 \pm 2.4$ & 0.017 & $50.7 \pm 3.0$ & $<.0001$ & $49.3 \pm 2.3$ & $<.0001$ \\
\hline 8 weeks & $57.1 \pm 2.3$ & $56.5 \pm 2.7$ & 0.011 & $54.8 \pm 3.4$ & 0.006 & $52.7 \pm 2.7$ & $<.0001$ \\
\hline 3 months & $60.4 \pm 2.3$ & $59.7 \pm 2.8$ & 0.005 & $57.4 \pm 3.5$ & $<.0001$ & $56.2 \pm 2.5$ & $<.0001$ \\
\hline 4 months & $63.7 \pm 2.4$ & $62.9 \pm 2.6$ & 0.003 & $61.0 \pm 3.9$ & 0.002 & $59.2 \pm 2.5$ & $<.0001$ \\
\hline 6 months & $67.8 \pm 2.5$ & $67.4 \pm 2.6$ & 0.135 & $64.3 \pm 4.2$ & 0.008 & $64.4 \pm 2.1$ & $<.0001$ \\
\hline 12 months & $75.2 \pm 2.5$ & $74.6 \pm 3.1$ & 0.038 & $71.9 \pm 4.3$ & 0.001 & $72.2 \pm 2.1$ & $<.0001$ \\
\hline 18 months & $82.8 \pm 3.2$ & $81.6 \pm 3.3$ & 0.036 & $78.5 \pm 4.4$ & 0.008 & $80.0 \pm 0.7$ & 0.034 \\
\hline 2 years & $89.0 \pm 3.4$ & $88.6 \pm 3.4$ & 0.394 & $84.5 \pm 4.4$ & 0.009 & $86.8 \pm 2.8$ & 0.024 \\
\hline 3 years & $97.6 \pm 3.8$ & $96.9 \pm 3.2$ & 0.263 & $90.3 \pm 3.9$ & 0.001 & $96.3 \pm 3.2$ & 0.261 \\
\hline 5 years & $103.9 \pm 4.8$ & $119.0 \pm 6.8$ & 0.155 & $112.4 \pm 4.5$ & 0.045 & $113.6 \pm 2.5$ & 0.041 \\
\hline \multicolumn{8}{|l|}{ Weight (kg) } \\
\hline 4 weeks & $4.3 \pm 0.5$ & $4.4 \pm 3.0$ & 0.759 & $3.4 \pm 0.6$ & 0.049 & $3.2 \pm 0.5$ & $<.0001$ \\
\hline 8 weeks & $5.2 \pm 0.6$ & $4.9 \pm 0.7$ & $<.0001$ & $4.3 \pm 0.8$ & $<.0001$ & $3.9 \pm 0.7$ & $<.0001$ \\
\hline 3 months & $6.1 \pm 0.7$ & $5.8 \pm 0.8$ & $<.0001$ & $5.1 \pm 0.9$ & $<.0001$ & $4.9 \pm 0.6$ & $<.0001$ \\
\hline 4 months & $6.9 \pm 0.8$ & $6.5 \pm 0.8$ & $<.0001$ & $5.7 \pm 1.1$ & $<.0001$ & $5.5 \pm 0.6$ & $<.0001$ \\
\hline 6 months & $8.0 \pm 0.9$ & $7.6 \pm 0.9$ & $<.0001$ & $6.6 \pm 1.2$ & $<.0001$ & $6.6 \pm 0.7$ & $<.0001$ \\
\hline 12 months & $9.8 \pm 1.0$ & $9.5 \pm 1.0$ & 0.008 & $8.3 \pm 1.2$ & $<.0001$ & $8.6 \pm 1.0$ & $<.0001$ \\
\hline 18 months & $11.6 \pm 1.4$ & $11.0 \pm 1.6$ & 0.02 & $9.9 \pm 1.2$ & 0.018 & $10.1 \pm 1.1$ & 0.011 \\
\hline 2 years & $13.2 \pm 1.5$ & $12.7 \pm 1.6$ & 0.013 & $11.3 \pm 1.4$ & 0.012 & $11.7 \pm 1.2$ & 0.001 \\
\hline 3 years & $15.6 \pm 1.9$ & $14.8 \pm 1.6$ & 0.01 & $12.4 \pm 1.8$ & 0.003 & $13.7 \pm 1.6$ & 0.001 \\
\hline 5 years & $24.0 \pm 2.8$ & $22.0 \pm 4.6$ & 0.572 & $20.0 \pm 1.9$ & 0.043 & $20.0 \pm 1.6$ & 0.043 \\
\hline \multicolumn{8}{|c|}{ Head circumference $(\mathrm{cm})$} \\
\hline 4 weeks & $37.1 \pm 1.3$ & $37.0 \pm 1.24$ & 0.519 & $37.1 \pm 1.1$ & 0.968 & $35.5 \pm 0.8$ & 0.007 \\
\hline 8 weeks & $38.8 \pm 1.36$ & $38.4 \pm 1.49$ & 0.151 & $39.1 \pm 1.4$ & 0.681 & $37.4 \pm 0.7$ & 0.032 \\
\hline 3 months & $40.3 \pm 1.36$ & $40.0 \pm 1.44$ & 0.066 & $40.7 \pm 1.0$ & 0.597 & $38.6 \pm 1.2$ & 0.001 \\
\hline 4 months & $41.7 \pm 1.35$ & $41.3 \pm 1.42$ & 0.054 & $42.2 \pm 1.1$ & 0.490 & $39.8 \pm 1.4$ & $<.0001$ \\
\hline 6 months & $43.6 \pm 1.48$ & $43.2 \pm 1.37$ & 0.110 & $44.1 \pm 0.8$ & 0.500 & $41.8 \pm 1.5$ & 0.001 \\
\hline
\end{tabular}

Data presented as mean \pm standard deviation. AGA: appropriate-for-gestational age. SGA: small-for-gestational age; ACv, abdominal circumference velocity between 20 and 32 weeks of gestational age; $A C v<25 \%$, abdominal circumference velocity lowest $25 \%$.

\section{Longitudinal measurements}

The second-order Reed model provided the best fit of the data both for height and weight (results not shown) and was subsequently used to test the hypothesis that growth would differ between SGA and AGA neonates. 
The model, including the SGA indicator as covariate showed better model fit compared to the model without covariates, indicated by a lower AIC. The interaction between SGA and age was not statistically significant (regression coefficient: $-0.07,95 \%$ confidence interval $[\mathrm{CI}]:-0.33 ; 0.18, \mathrm{p}=0.581$ ). Hence, the final model for height was reduced to include only the covariate for SGA. The regression coefficient of SGA was $-2.9 \mathrm{~cm}(95 \% \mathrm{CI}:-2.3 ;-3.6, \mathrm{p}<0.001)$ meaning that, on average, SGA neonates were $2.9 \mathrm{~cm}$ shorter than their AGA counterparts over the course of follow-up, with no significant evidence that the difference changes considerably over time. Figure 1 shows the observed growth trajectories of height over time and the predicted trajectories stratified by SGA and AGA.

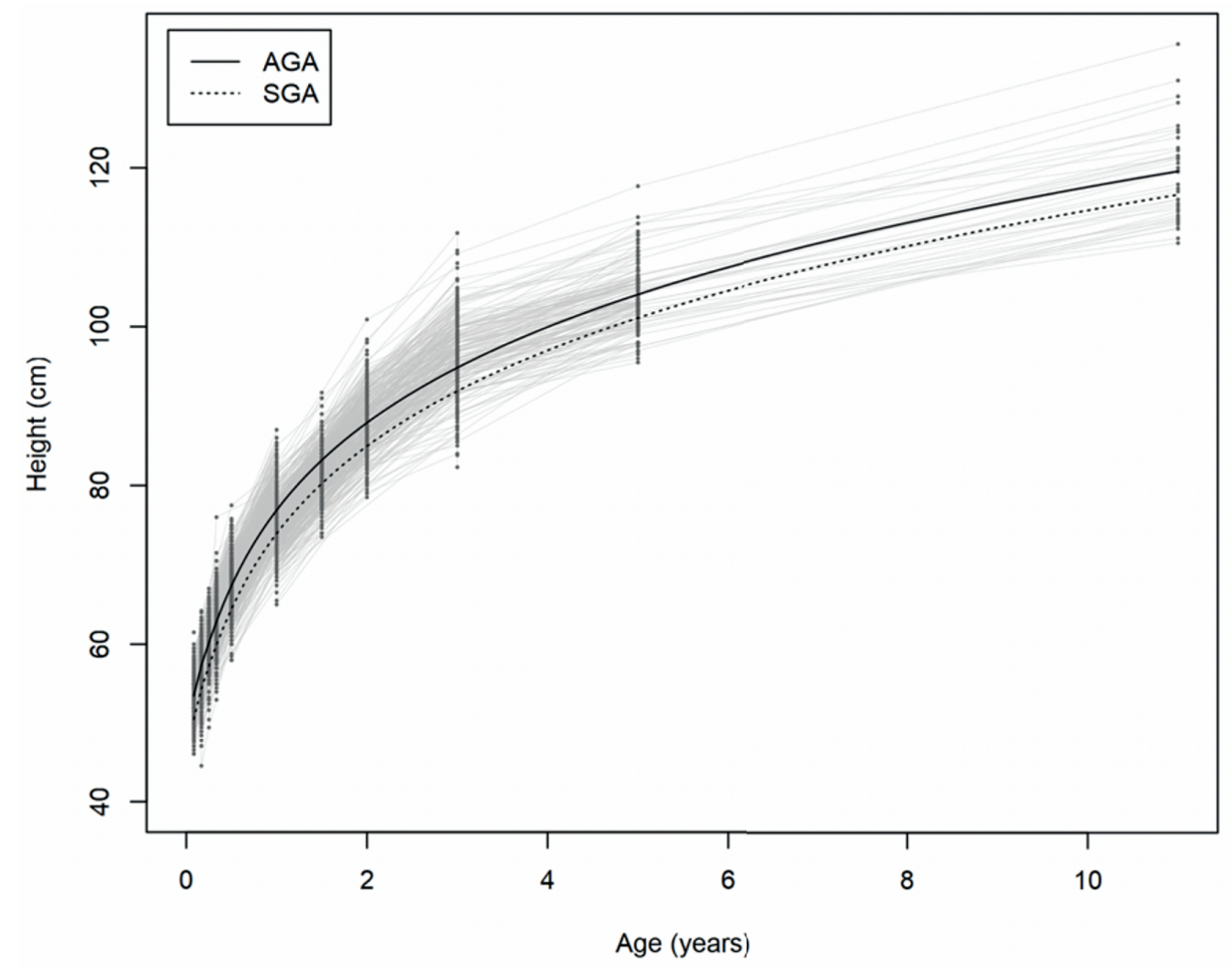

Figure 1 Postnatal observed growth trajectories of height $(\mathrm{cm})$ in SGA and AGA neonates

The weight of SGA group over the course of follow-up was $0.84 \mathrm{~kg}$ lower than the AGA group (95\% CI: $0.68 ; 1.00, \mathrm{p}<0.001$ ), and the model including the SGA indicator as covariate showed better model fit compared to only the growth parameters of the second-order Reed model. The interaction between age and SGA was statistically significant for the model of weight over time, indicating that the slope of SGA neonates of weight over time differs from AGA neonates 
(regression coefficient: -0.16 (95\% CI: $-0.30,-0.01, \mathrm{p}=0.033)$. Figure 2 shows the observed growth trajectories of weight over time and the predicted trajectories stratified by SGA and AGA.

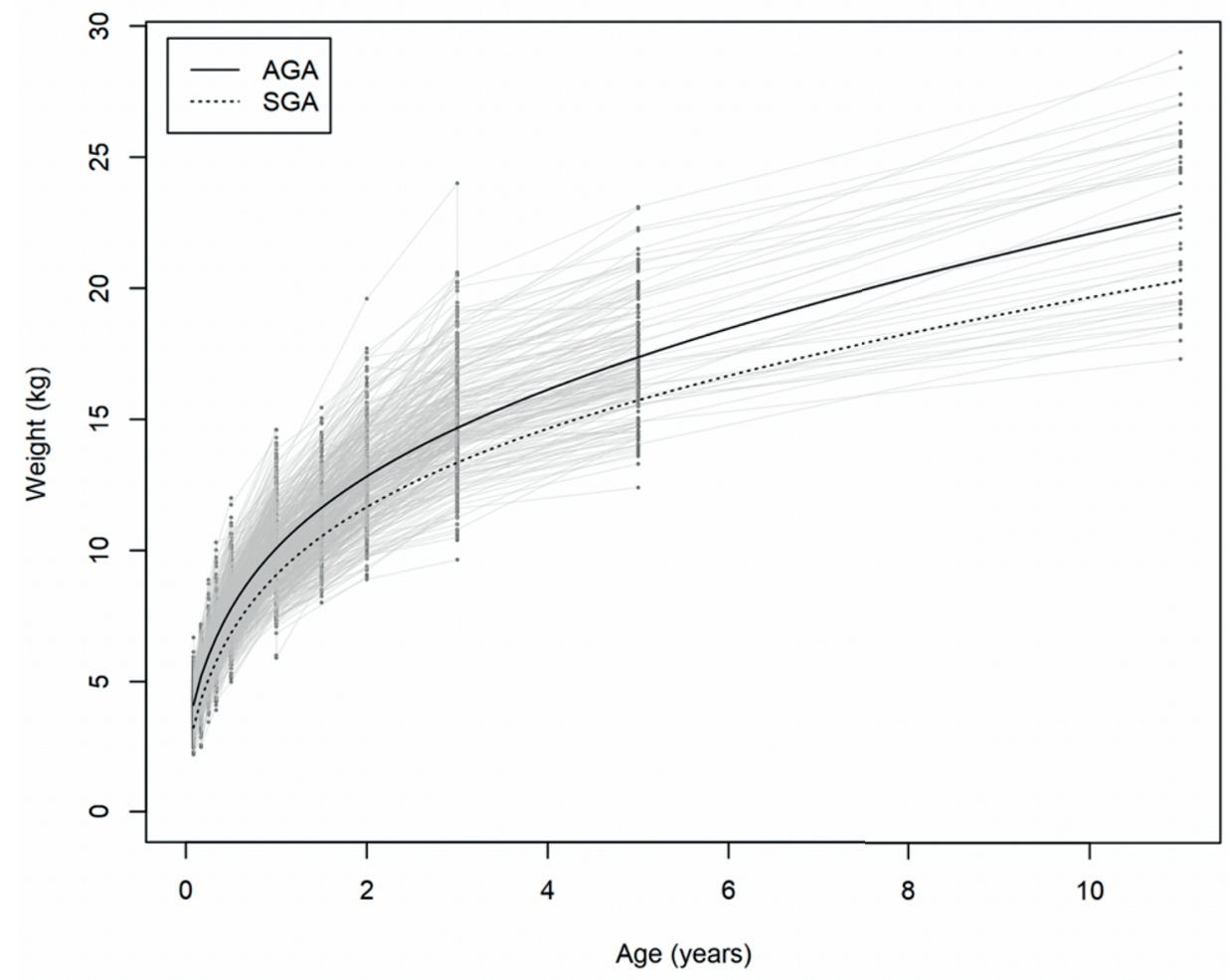

Figure 2 Postnatal observed growth trajectories of weight (kg) in SGA and AGA neonates

\section{Catch-up growth up to and until 3 years after birth}

SGA and AGA neonates with a reduced fetal growth velocity had a significant higher risk showing catch-up growth in height compared with normal growing fetuses $(\mathrm{OR}=3.16$ (95\% CI 1.28-7.80)). The risk of catch-up growth in weight was not significant increased between both groups ( $\mathrm{OR}=1.269$ (94\% CI 0.47-3.43)). The absolute increase in the growth parameters height and weight between 4 weeks and 3 years, was compared between the SGA and AGA groups (Table 4). SGA with a reduced growth velocity (SGA, ACv <25\%) had a significantly higher catchup growth based on height (51.0 vs 44.8 ) compared with the reference group $(\mathrm{p}=0.042$ ) between 4 weeks and 3 years. No significant differences were found between the subgroups in catch-up growth based on weight. 
Table 4. Catch-up growth between 4 weeks and 3 year after birth in AGA and SGA neonates. AGA $\mathrm{ACv}>25 \%$ as reference group.

\begin{tabular}{|c|c|c|c|c|c|c|c|}
\hline \multirow[t]{2}{*}{ Catch-up } & \multicolumn{7}{|c|}{$4 w k s-3$ year $(n=229)$} \\
\hline & $\begin{array}{c}\text { AGA+ ACv }>25 \% \\
n=170 \\
\text { (reference) }\end{array}$ & $\begin{array}{c}\text { AGA+ ACv } \\
<25 \% \\
n=45\end{array}$ & p-value & $\begin{array}{c}S G A+A C v \\
>25 \% \\
n=3\end{array}$ & p-value & $\begin{array}{c}\text { SGA + ACv } \\
<25 \% \\
n=11\end{array}$ & p-value \\
\hline Height delta & $44.8 \pm 8.1$ & $47.8 \pm 13.4$ & 0.069 & $41.8 \pm 3.4$ & 0.596 & $51.0 \pm 15.4$ & 0.042 \\
\hline Weight delta & $11.4 \pm 1.9$ & $11.1 \pm 1.4$ & 0.365 & $9.5 \pm 1.6$ & 0.083 & $10.7 \pm 2.1$ & 0.264 \\
\hline
\end{tabular}

Data presented as mean \pm standard deviation; AGA, appropriate-for-gestational age; SGA, small-for-gestational age; ACv, abdominal circumference velocity between 20 and 32 weeks of gestational age; ACv $<25 \%$, abdominal circumference velocity lowest 25\%. Catch-up growth defined as the increase in height and weight between 4 weeks and 3 years after birth.

\section{DISCUSSION}

The data of the present study show that the SGA neonates had a significantly lower height, weight and head circumference compared to the AGA group at most individual time points in the first years after birth. The possible catch-up growth was evaluated by using delta height and weight values in the period of 4 weeks to 3 years. Fetuses with reduced fetal growth velocity, irrespective of birthweight, had a significant higher risk for catch-up growth in height 3 years after birth.

Our first results are based on a heterogeneous group of SGA fetuses including both FGR and constitutionally small fetuses. Heterogeneity in study groups is an occurring problem in a lot of growth studies [35, 36]. Postnatal growth trajectories may differ for the two types of SGA fetuses. Therefore, fetal growth velocity was added in the present study to distinguish fetal growth from fetal size. Our data suggest a possible link between fetal growth velocity and postnatal catch-up growth and support the idea of Beltrand et al., that early catch-up growth is more likely linked to fetal growth velocity, irrespective to birth weight [18, 37, 38]. The study of Beukers et al. also showed that the degree of placental insufficiency in FGR has a positive significant association with growth parameters at 4.5 years [39]. Neonates with a decelerating growth curve between second trimester and birth, have an increased risk of postnatal accelerated growth, independent of birthweight category (SGA or AGA) [19].

There is still no convincing evidence for the exact mechanism involved in catch-up growth. Two models have been proposed: the neuroendocrine model and the growth plate model [40]. According to the neuroendocrine model stated above, the body size is compared to an age appropriate set point. If a mismatch is detected, the growth rate will adjust accordingly. The growth plate model, on the other hand, suggests a more local mechanism. According to this model, growth- 
inhibiting conditions decrease proliferation of growth plate stem cells. After cessation of the growth arrest the number of divisions of these stem cells is lower than expected. The cells begin to proliferate at a faster rate than the non-exposed cells and results in local catch-up growth [10, 41]. The growth plate model could explain the difference that only fetuses with reduced growth velocity, which are probably the 'truly' intra-uterine growth restricted fetuses show postnatal catch up growth, while small fetuses with normal growth had no catch up.

Catch-up growth can be either complete or incomplete, according to the study of Wit et al. Catch-up growth is considered to be complete in case the mean final height is reached close to the mean target height of both parents. In case of incomplete catch-up growth, the target height range will not be reached and this is associated with increased neurodevelopmental problems when compared to infants with complete catch-up [10]. In contradiction, the study of van Wijk et al. showed that neonates with catch-up growth were associated with neurodevelopmental problems at 2 years of age [42]. It is still unclear if catch-up growth in height is positive or not according to neurodevelopment.

In this study, neonates with a normal intra uterine growth velocity showed less increase in postnatal height (regardless of their birthweight), compared with neonates with a reduced growth velocity. Studies showed that fetuses with a lower height at 2 years, remain short in adult stature, whether or not born as SGA [43]. Being born short or lagging in height during the first years of life is similar in terms of increased risk for adult shortness [35]. Children born growth restricted due to early onset placental insufficiency showed growth patterns comparable to normal birth weight fetuses at age of 12.5 years, suggesting environmental and genetic factors overshadow fetal factors of growth [39].

Our data can have important consequences in the context of management of neonatal with pathological impairment of growth, both height and weight. Short infants who meet the definition criteria at birth and fail to demonstrate catch-up growth by 2-4 years based on height of age are currently considered candidates for growth hormone (GH) treatment. GH treatment is initiated to achieve fortified growth to a normal height in early childhood, maintain a normal height gain throughout childhood, and achieve an adult height within the normal target range $[44,45]$.

SGA neonates born with a short stature usually do not have a classical GH deficiency, but instead have been found to have either low GH secretion or reduced sensitivity to GH [46]. However, the response to growth hormone treatments may be different in the SGA and reduced growth velocity groups in this study. It is not clear whether SGA and AGA short infants have similar responses to the same dose of growth hormone treatment. Some authors have suggested that neonates born 
SGA might need a higher dose of growth hormone to achieve a desirable growth response [47]. Taken together, our data highlight the relevance of taking fetal growth velocity into account in the management of neonates with suspected fetal growth restriction and fit integrally within the concept of personalised preventive medicine in an attempt to reduce the health burden for future generations.

\section{Strengths and limitations}

This study is one of the first studies that examines the relationship between intrauterine fetal growth velocity and postnatal catch-up growth expressed in height and weight. One of the strengths is that this study focused at growth restriction and catch-up growth in both SGA and AGA neonates. Another strength of this study is the fact that there is arguably less variance in infant biometric measurements than previous studies using conventional methods, as measurements in our study were performed by experienced assistants in dedicated community centres and the data being recorded according to standardised protocols. The main limitation of this study is its retrospective setup that led to incomplete data collection for some participants at some measurement moments, leading to a risk of selection bias. Another limitation is the missing data of different factors determining postnatal growth including paternal characteristics, type of feeding, economic status and maternal education levels [48].

\section{CONCLUSION}

This study shows that neonates who are born small (less than the $10^{\text {th }}$ centile for gestational age) had significantly lower postnatal growth trajectories of their height, weight and head circumference compared to AGA neonates during the first years of life. On the other hand, fetuses with growth restriction intra-uterine (defined by smaller fetal ultrasound parameters or reduced growth velocity) are at a significant risk for developing catch-up growth mainly in height after birth. This suggests a role for fetal growth velocity in identifying fetuses at risk for fetal growth restriction and its postnatal complications and consequently health risks in adulthood. A large prospective study is needed to determine if implementation of fetal growth velocity in clinical practise is indeed beneficial in identifying neonates at high risk and could lead to improved long-term health outcomes. 


\section{REFERENCES}

1. Sharma, D., S. Shastri, and P. Sharma, Intrauterine Growth Restriction: Antenatal and Postnatal Aspects. Clin Med Insights Pediatr, 2016. 10: p. 67-83.

2. Gordijn, S., et al., Consensus definition of fetal growth restriction: a Delphi procedure. Ultrasound in Obstetrics \& Gynecology, 2016. 48(3): p. 333-339.

3. Sharma, D., P. Sharma, and S. Shastri, Postnatal Complications of Intrauterine Growth Restriction. Journal of Neonatal Biology, 2016. 05(04).

4. Stock, S.J. and J. Myers, Defining Abnormal Fetal Growth and Perinatal Risk: Population or Customized Standards? PLoS Med, 2017. 14(1): p. e1002229.

5. Lees, C., et al., Perinatal morbidity and mortality in early-onset fetal growth restriction: cohort outcomes of the trial of randomized umbilical and fetal flow in Europe (TRUFFLE). Ultrasound Obstet Gynecol, 2013. 42(4): p. 400-8.

6. Salam, R.A., J.K. Das, and Z.A. Bhutta, Impact of intrauterine growth restriction on longterm health. Curr Opin Clin Nutr Metab Care, 2014. 17(3): p. 249-54.

7. MacDonald, T.M., et al., Reduced growth velocity across the third trimester is associated with placental insufficiency in fetuses born at a normal birthweight: a prospective cohort study. BMC Med, 2017. 15(1): p. 164.

8. Mayer, C. and K.S. Joseph, Fetal growth: a review of terms, concepts and issues relevant to obstetrics. Ultrasound Obstet Gynecol, 2013. 41(2): p. 136-45.

9. Ananth, C.V. and A.M. Vintzileos, Distinguishing pathological from constitutional small for gestational age births in population-based studies. Early human development, 2009. 85(10): p. 653-658.

10. Wit, J.M. and B. Boersma, Catch-up growth: definition, mechanisms, and models. J Pediatr Endocrinol Metab, 2002. 15 Suppl 5: p. 1229-41.

11. Singhal, A., Should We Promote Catch-Up Growth or Growth Acceleration in LowBirthweight Infants? Nestle Nutr Inst Workshop Ser, 2015. 81: p. 51-60.

12. Cho, W.K. and B.-K. Suh, Catch-up growth and catch-up fat in children born small for gestational age. Korean journal of pediatrics, 2016. 59(1): p. 1.

13. Gibson, A.T., et al., Neonatal and post-natal growth. Horm Res, 2000. 53 Suppl 1: p. 42-9.

14. Albertsson-Wikland, K. and J. Karlberg, Natural growth in children born SGA with and without catch up growth. Horm Res, 2003. 59 Suppl 1: p. 129.

15. Albertsson-Wikland, K. and J. Karlberg, Postnatal growth of children born small for gestational age. Acta Paediatr Suppl, 1997. 423: p. 193-5.

16. Hwang, I.T., Efficacy and safety of growth hormone treatment for children born small for gestational age. Korean J Pediatr, 2014. 57(9): p. 379-83.

17. Lee, S.M., et al., Prediction of Postnatal Growth Failure among Very Low Birth Weight Infants. Sci Rep, 2018. 8(1): p. 3729.

18. Beltrand, J., et al., Catch-up growth following fetal growth restriction promotes rapid restoration of fat mass but without metabolic consequences at one year of age. PLoS One, 2009. 4(4): p. e5343.

19. Broere-Brown, Z.A., et al., Deceleration of fetal growth rate as alternative predictor for childhood outcomes: a birth cohort study. BMC Pregnancy and Childbirth, 2019. 19(1): p. 216.

20. Lee, S.M., et al., Prediction of postnatal growth failure among very low birth weight infants. Scientific reports, 2018. 8(1): p. 1-8. 
21. Zhang, L., et al., Postnatal length and weight growth velocities according to Fenton reference and their associated perinatal factors in healthy late preterm infants during birth to term-corrected age: an observational study. Italian journal of pediatrics, 2019. 45(1): p. 1.

22. Robinson, H.P., Sonar measurement of fetal crown-rump length as means of assessing maturity in first trimester of pregnancy. Br Med J, 1973. 4(5883): p. 28-31.

23. Salomon, L.J., et al., Practice guidelines for performance of the routine mid-trimester fetal ultrasound scan. Ultrasound Obstet Gynecol, 2011. 37(1): p. 116-26.

24. Caradeux, J., et al., Second- to third-trimester longitudinal growth assessment for prediction of small-for-gestational age and late fetal growth restriction. Ultrasound Obstet Gynecol, 2018. 51(2): p. 219-224.

25. Sovio, U. and G.C.S. Smith, Screening performance of consensus definition of fetal growth restriction inappropriately evaluated - Authors' reply. Lancet Child Adolesc Health, 2018. 2(9): p. e23.

26. Hadlock, F.P., et al., Estimation of fetal weight with the use of head, body, and femur measurements--a prospective study. Am J Obstet Gynecol, 1985. 151(3): p. 333-7.

27. Hendrix, M.L.E., et al., Reduced fetal growth velocities and the association with neonatal outcomes in appropriate-for-gestational-age neonates: a retrospective cohort study. BMC Pregnancy Childbirth, 2019. 19(1): p. 31.

28. Hendrix, M.L.E., et al., Can Fetal Growth Velocity and First Trimester Maternal Biomarkers Improve the Prediction of Small-for-Gestational Age and Adverse Neonatal Outcome? Fetal Diagn Ther, 2019. 46(4): p. 274-284.

29. Francis, A.H., O; Gardosi, J, Slow growth defined by customised growth trajectory and adverse perinatal outcome. International Conference of Fetal Growth, 2019.

30. Iliodromiti, S., et al., Customised and Noncustomised Birth Weight Centiles and Prediction of Stillbirth and Infant Mortality and Morbidity: A Cohort Study of 979,912 Term Singleton Pregnancies in Scotland. PLoS Med, 2017. 14(1): p. e1002228.

31. Touwslager, R.N., et al., Longitudinal growth during the first years of life: what is normal? Horm Res, 2008. 70(5): p. 273-7.

32. Gerver, W. and R. De Bruin, Paediatric morphometrics. Universitaire Pers Maastricht, Maastricht, 2001: p. 48-51.

33. de Wit, C.C., et al., Patterns of catch-up growth. J Pediatr, 2013. 162(2): p. 415-20.

34. Berkey, C.S. and R.B. Reed, A model for describing normal and abnormal growth in early childhood. Human Biology, 1987: p. 973-987.

35. Luo, Z.C., K. Albertsson-Wikland, and J. Karlberg, Length and body mass index at birth and target height influences on patterns of postnatal growth in children born small for gestational age. Pediatrics, 1998. 102(6): p. E72.

36. Finken, M.J., et al., Long-term height gain of prematurely born children with neonatal growth restraint: parallellism with the growth pattern of short children born small for gestational age. Pediatrics, 2006. 118(2): p. 640-3.

37. Weber, M., et al., Lower protein content in infant formula reduces BMI and obesity risk at school age: follow-up of a randomized trial. Am J Clin Nutr, 2014. 99(5): p. 1041-51.

38. Hokken-Koelega, A.C., et al., Children born small for gestational age: do they catch up? Pediatr Res, 1995. 38(2): p. 267-71.

39. Beukers, F., et al., Catch-up growth in children born growth restricted to mothers with hypertensive disorders of pregnancy. Arch Dis Child, 2013. 98(1): p. 30-5. 
40. Gafni, R.I. and J. Baron, Catch-up growth: possible mechanisms. Pediatr Nephrol, 2000. 14(7): p. 616-9.

41. Boersma, B. and J.M. Wit, Catch-up growth. Endocr Rev, 1997. 18(5): p. 646-61.

42. van Wyk, L., et al., Postnatal Catch-Up Growth After Suspected Fetal Growth Restriction at Term. Front Endocrinol (Lausanne), 2019. 10: p. 274.

43. Jaquet, D., et al., Adult height distribution in subjects born small for gestational age. Horm Res, 2004. 62(2): p. 92-6.

44. Reiter, E.O., et al., Effect of growth hormone (GH) treatment on the near-final height of 1258 patients with idiopathic GH deficiency: analysis of a large international database. J Clin Endocrinol Metab, 2006. 91(6): p. 2047-54.

45. Darendeliler, F., A. Lindberg, and P. Wilton, Response to growth hormone treatment in isolated growth hormone deficiency versus multiple pituitary hormone deficiency. Horm Res Paediatr, 2011. 76 Suppl 1: p. 42-6.

46. de Waal, W.J., et al., Endogenous and stimulated GH secretion, urinary GH excretion, and plasma IGF-I and IGF-II levels in prepubertal children with short stature after intrauterine growth retardation. The Dutch Working Group on Growth Hormone. Clin Endocrinol (Oxf), 1994. 41(5): p. 621-30.

47. Chatelain, P., et al., Growth hormone therapy for short children born small for gestational age. Horm Res, 2007. 68(6): p. 300-9.

48. Ozanne, S.E. and C.N. Hales, Lifespan: catch-up growth and obesity in male mice. Nature, 2004. 427(6973): p. 411-2. 

c) 
General discussion

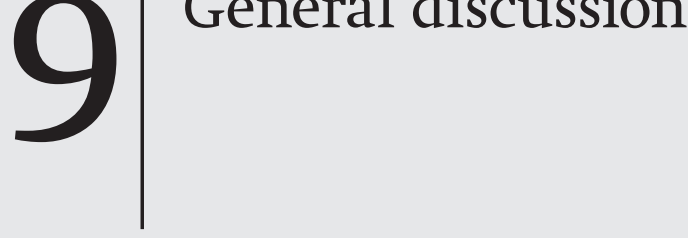





\section{Contemporary view on current definition of fetal growth restriction}

Human growth, both during intrauterine and postnatal life, is an intricate and dynamic process that incorporates the effects of genetic and environmental factors, most of which are not adequately recognized [1, 2]. After decades of clinical research, it has become clear that pathological failure of a fetus to reach its growth potential is the underlying cause of the clinically prevalent disorder called fetal growth restriction (FGR) [3, 4]. It is not difficult to imagine that a single momentary ultrasound measurement is not sufficient to grasp the complexity of the fetal growth process in utero, nor to diagnose its pathological variant, FGR. The research described in this thesis has focused on measuring fetal growth velocities through the third trimester of pregnancy as a potentially new method to identify truly growth restricted fetuses due to placental dysfunction. In addition, this approach will identify a subgroup of small babies with normal growth velocity and no underlying placental dysfunction and are, hence, at less risk of neonatal morbidity.

Recently, a new consensus definition for defining fetal growth restriction has been published, which for the first time took into consideration the dynamic aspect of fetal growth. This consensus definition was based on the Delphi method using a survey of 45 experts on a wide range of clinical, ultrasound and biochemical parameters linked to fetal growth restriction [5]. This guideline introduced "crossing centiles" as one of the new parameters, as a surrogate for reduced growth velocity (Table 1). In this guideline, FGR is defined as fetal abdominal circumference (AC) or estimated fetal weight (EFW) below the $3^{\text {rd }}$ centile; or an AC or EFW below the $10^{\text {th }}$ centile with crossing centiles $>2$ quartiles on growth centiles; and/or an abnormal doppler profile (cerebroplacental ratio $(\mathrm{CPR})<5^{\text {th }}$ or Umbilical Artery Pulsatility Index (UA-PI) $>95^{\text {th }}$ centile). As a consequence, a fetus with an AC/EFW above the $10^{\text {th }}$ centile, but crossing centiles $>2$ quartiles and an abnormal doppler profile, can be defined as late FGR. The use of crossing centiles as a parameter for reduced growth, will reclassify babies that would have otherwise been considered having appropriate growth, into a new category of FGR. This supports the idea that even an AGA fetus ( $>10^{\text {th }}$ centile) can be truly growth restricted, and highlights the additional value of calculating growth velocity described in this thesis. A lot of recent publications about fetal growth use the consensus definition as outcome measurement for FGR [6, 7]. Using the same definition for FGR makes it possible to compare and combine study results better and possibly reduce adverse outcomes [5]. 
Table 1. Consensus definition for FGR based on a Delphi procedure, Gordijn et al (1).

\begin{tabular}{|c|c|}
\hline $\begin{array}{c}\text { Early FGR: GA }<32 \text { weeks, in absence of } \\
\text { congenital anomalies }\end{array}$ & $\begin{array}{c}\text { Late FGR: GA } \geq 32 \text { weeks, in absence of congenital } \\
\text { anomalies }\end{array}$ \\
\hline $\mathrm{AC} / \mathrm{EFW}<3^{\text {rd }}$ centile or UA-AEDF & $\mathrm{AC} / \mathrm{EFW}<3^{\text {rd }}$ centile \\
\hline Or & Or at least two out of three of the following \\
\hline 1. $\mathrm{AC} / \mathrm{EFW}<10^{\text {th }}$ centile combined with & 1. $\mathrm{AC} / \mathrm{EFW}<10^{\text {th }}$ centile \\
\hline 2. UtA-PI $>95^{\text {th }}$ centile and/or & $\begin{array}{l}\text { 2. AC/EFW crossing centiles }>2 \text { quartiles on growth } \\
\text { centiles* }\end{array}$ \\
\hline 3. UA-PI $>95^{\text {th }}$ centile & 3. $\quad \mathrm{CPR}<5^{\text {th }}$ centile or UA-PI $>95$ \\
\hline
\end{tabular}

AC, abdominal circumference; AEDF, absent end-diastolic flow; CPR, cerebroplacetal ratio; EFW, estimated fetal weight; GA, gestational age; PI, pulsatility index; UA, umbilical artery; UtA, uterine artery. * growth centiles are non-customized centiles.

\section{Phenotypes of fetal growth}

It is obvious from the mounting recent literature on the variable clinical presentation of FGR: in severity [8], time from onset of disease [9, 10], doppler assessments $[11,12]$ and placental pathology [13], that FGR is not a simple homogenous disease, but a complex heterogenous syndrome with at least two phenotypes. In view of the new consensus definition [5] and the data presented in this thesis, combined with the current knowledge about angiogenic biomarkers and placental histology, we suggest a classification of four conceptual phenotypes of fetal growth patterns, as displayed in figure 1 .

- Normal-Growing (NG) (green): the majority of fetus will be appropriately growing, and will follow their own growth trajectory during pregnancy spanning the $10^{\text {th }}-90^{\text {th }}$ percentiles of the reference growth curve. There is a three-phase growth during different gestational intervals: a slow rise till 20-24 weeks, followed by an acceleration phase until 34 weeks, and a slow decline in growth velocity afterwards. The normally growing fetus will have a birthweight between the $10^{\text {th }}-90^{\text {th }}$ percentiles for the reference birth register, has a normal perinatal transition and is at the lowest risk of stillbirths and neonatal mortality. The placental weight is normal $\left(10^{\text {th }}-90^{\text {th }}\right.$ percentiles $)$ and there is no significant placental pathology on macroscopic or histological examination. There are normal Doppler findings of uterine, placental and fetal blood vessels. The placental function is normal as reflected by normal levels of placental hormones and angiogenic biomarkers. Identifying the normally growing fetus will allow a minimum set of antenatal visits and efficient use of obstetric resources for monitoring the higher risk fetus. There is no need for elective delivery in the absence of other obstetric complications and expectant management of the later stages of pregnancy is appropriate. 


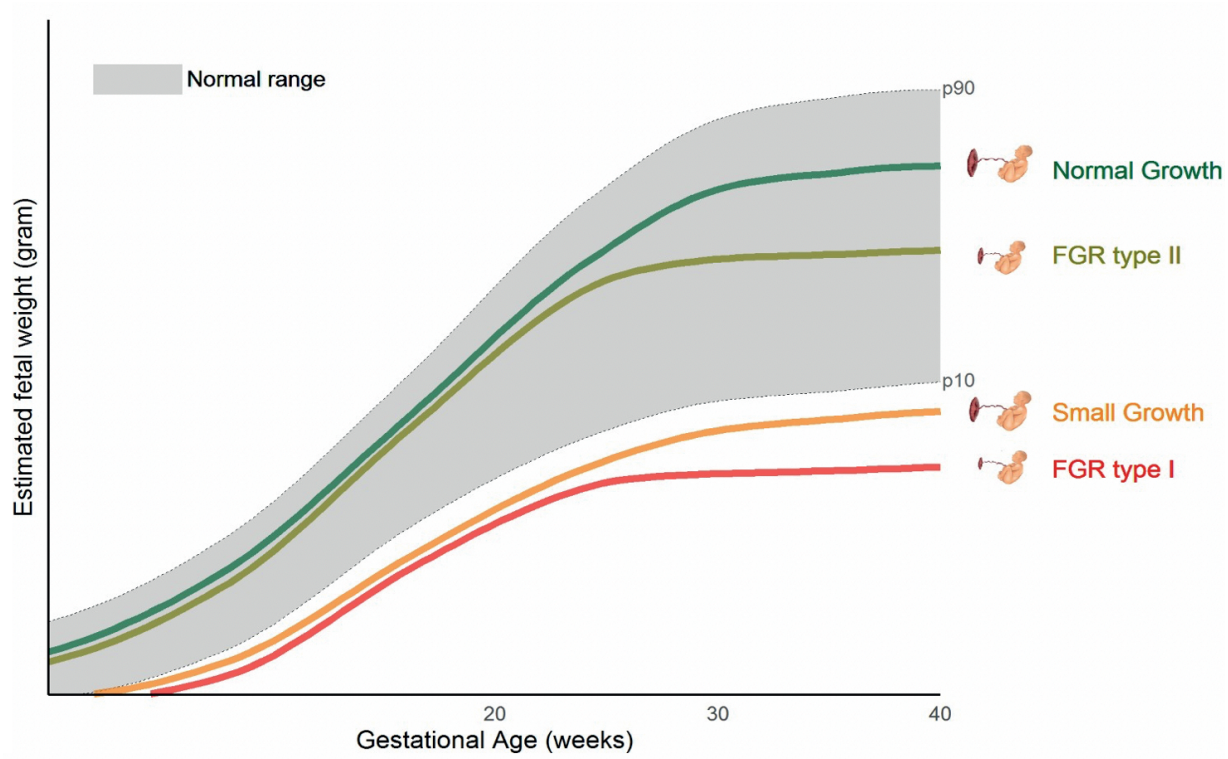

Figure 1 Theoretical depiction of fetal growth trajectories in four proposed phenotypes of fetal growth

FGR type I, small-for-gestational age with fetal growth restriction (red), constitutional small growth (orange). FGR type II, appropriate-for-gestational age with fetal growth restriction (yellow), normal growth (green). The $x$-axis presents the gestational age in weeks. The $y$-axis presents estimated fetal weight in gram.

- Small-Growing (SG) (orange): this constitutionally small fetus will characteristically follow its own growth trajectory during pregnancy parallel, but at the lower end of the reference growth curve. There is a stable growth during time without decline in growth velocity between 20-34 weeks. This fetus is constitutionally and symmetrically small as dictated by genetic determinants of fetal growth and placenta function, reflecting the influence of parental height and ethnicity. The constitutional small fetus will have a birthweight below the $10^{\text {th }}$ percentile for reference birth registers, but has a low risk of stillbirth and perinatal mortality $[14,15]$. The placental size is smaller than average, but there is no significant placental pathology on macroscopic or histological examination $[16,17]$. There are normal Doppler findings of uterine, placental and fetal blood vessels. The placental function is normal as reflected by normal levels of placental hormones and angiogenic biomarkers. Identifying the constitutional small fetus is important in order to plan a suitable monitoring strategy and prevent unnecessary obstetric interventions leading to iatrogenic preterm delivery. 
- FGR type I (red): This phenotype is the equivalent to the classical severe early FGR, representing the epitome of placental insufficiency. This fetus will show an early decline in growth trajectories, evident even in the second trimester. First trimester screening with uterine Doppler velocimetry, angiogenic markers and maternal characteristics may detect FGR type I in up to 90\% [18]. The typical pattern of deterioration progresses from escalating abnormalities in Doppler parameters to abnormal biophysical parameters. The umbilical artery Doppler (UA) can be used to detect fetuses with severe placental disease [19]. A lower increase in PIGF and a higher increase in sFLt-1 compared with normal growing fetus is seen (chapter 4) [20,21]. Placental histology shows prominent and diverse vascular and parenchymal lesions collectively known as maternal vascular malperfusion (MVM) lesions [22]. These MVM lesions are thought to be the consequence of the process of defective remodeling of the maternal spiral arteries [23-25]. The placenta often has a reduction in volume, surface area, and vascularization of the intermediate and terminal villi that mediates maternal-fetal exchange. This reduction appears to be due to excessive villous regression during placental remodeling, compounded by a reduced rate of subsequent placental growth [13]. This type of FGR presents in association with early preeclampsia (PE) in up to 50\% [26]. A long period of close fetal monitoring is often necessary to observe early signs of fetal deterioration and complete corticosteroid treatment for lung maturation. This fetus is almost always born preterm and has a birthweight below the $10^{\text {th }}$ centile, more often below the $3^{\text {rd }}$ centile. Elective preterm delivery is usually indicated in the second or early third trimester as dictated by abnormal fetal heart recording or severe Doppler abnormalities, or by the development of maternal complications, such as preeclampsia. This type of FGR has the highest association with poor perinatal outcomes, either in the form of fetal deaths or neonatal mortality and morbidity [27]. Although detection is relatively simple, management is very challenging and aims at achieving the best balance between the risk of stillbirth and complications of prematurity [10]. The use of angiogenic biomarkers and doppler assessment can be helpful in discriminating the fetus with placental dysfunction from the constitutional small fetus in order to reduce the risk of stillbirth [28].

- FGR type II (yellow): This phenotype of FGR is the most obscure and difficult to detect, yet as severe and relevant to perinatal mortality as FGR type I. This fetus has a normal growth pattern in the first half of pregnancy, followed by a decline (or suboptimal rise) in growth velocity in third trimester. Histological signs of placental under perfusion (mainly vascular occlusion and villous hy- 
poplasia) are seen, but are less pronounced compared to FGR type I. Umbilical artery (UA) Doppler often fails to pick up the possibly milder placental disease in this late-onset group [12]. However, altered fetal hemodynamic adaptation to placental under perfusion is apparent by a higher flow and reduced resistance in fetal middle cerebral artery (MCA). The cerebroplacental ratio (CPR) improves the detection of FGR-II using the increased placental impedance (UA) combined with reduced cerebral resistance (MCA). The CPR has been demonstrated to be more sensitive to hypoxia and correlates better with adverse outcomes in FGR at any gestational age [29]. Angiogenic biomarkers can be helpful in revealing subtle abnormalities in placental development associated with FGR-II. Lower PAPP-A levels during first trimester compared with normal growing fetuses are seen (chapter 4). Later in pregnancy is a higher sFlt-1/P1GF ratio associated with late FGR [6]. Placental histology displays less prominent placental dysfunction than FGR-I, however maternal vascular malperfusion (MVM) lesions can often be seen in the placenta of these fetuses [30, 31] with reduced growth (chapter 6) . There is a low association with PE, approximately $10 \%$ [18]. The clinical and ultrasound presentation of FGR type II is strikingly variable and consequently the detection of this condition, especially in the late-onset form, remains a major challenge in clinical practice. This variability is largely caused by the heterogeneity in type, severity, and progression of the underlying placental disease and is combined with the intrinsic variability of the fetus to adapt. These FGR fetuses are vulnerable to cerebral lesions resulting from hypoxia, due to the increasing oxygen requirements of the fetal brain near term [32]. Progression to severe fetal deterioration and even fetal death can occur rapidly. This type of FGR is associated with cesarean delivery for fetal distress, neonatal acidosis, and admission to the neonatal unit. Identification of this fetus by reduced growth velocity (or other parameters of suboptimal growth such as crossing centiles) will allow frequent monitoring and the ancillary use of Doppler to select the subgroup that would potentially benefit from induction of labor at term, without an increase in adverse neonatal outcomes [33].

It is important to note that this classification of fetal growth into four phenotypes focuses on identifying fetus with FGR and deliberately avoids fetus with accelerated growth as this falls beyond the scope of this thesis. It is probably also an oversimplification of the complex clinical syndrome of FGR, as the dichotomous division of FGR into only two subtypes ignores a possible overlap and shared mechanisms of placental dysfunction underlying both subtypes. 
Table 2 summarizes the differences in key characteristics between the four proposed phenotypes of fetal growth. The two phenotypes of physiological fetal growth differ mainly in the neonatal and placental weight, but not in features histology and biomarkers reflecting normal placental function or fetal hemodynamic adaptation. Neonatal outcome is usually normal in both groups, except in small-growing cases were birthweight is <3rd centile [14, 15], although this could reflect misdiagnosis of late FGR (FGR-II), because most studies do not utilize growth velocities or biomarkers of placental function.

Table 2. Differences in key characteristics between the four proposed phenotypes of fetal growth.

\begin{tabular}{|c|c|c|c|c|}
\hline & \multicolumn{2}{|c|}{ Physiological } & \multicolumn{2}{|c|}{ Pathological } \\
\hline & Normal Growth & Small Growth & FGR I & FGR II \\
\hline Fetal growth velocity & $\mathrm{nl}$ & $\mathrm{nl}$ & $\downarrow$ & $\downarrow$ \\
\hline Birthweight percentile & $\mathrm{nl}$ & $\downarrow$ & $\downarrow$ & $\mathrm{nl}$ \\
\hline Neonatal outcome & $\mathrm{nl}$ & $\begin{array}{c}\mathrm{nl} \\
\text { except if birthweight }<p 3\end{array}$ & $\begin{array}{c}\text { abnl } \\
\text { predictable }\end{array}$ & $\begin{array}{c}\text { abnl } \\
\text { unpredictable }\end{array}$ \\
\hline Placental weight & $\mathrm{nl}$ & $\downarrow$ & $\downarrow$ & $\mathrm{nl}$ \\
\hline Placental histology & $\mathrm{nl}$ & $\begin{array}{c}\mathrm{nl} \\
\text { or mild abnormal }\end{array}$ & $\begin{array}{c}\text { abnl } \\
\text { severe } M V M+F V M\end{array}$ & $\begin{array}{l}\text { abnl } \\
\text { mild FVM }\end{array}$ \\
\hline Doppler & $\mathrm{nl}$ & $\mathrm{nl}$ & $\begin{array}{c}\text { abnl } \\
\text { severe uterien/fetal }\end{array}$ & $\begin{array}{l}\text { abnl } \\
\text { mild fetal }\end{array}$ \\
\hline \multirow[t]{2}{*}{ Placental biomarkers } & $\mathrm{nl}$ & $\begin{array}{l}\mathrm{nl} \\
\text { or mild abnormal, related to placental mass }\end{array}$ & $\begin{array}{c}\text { abnl } \\
\text { severe, early 1st trimester }\end{array}$ & $\begin{array}{l}\text { abnl } \\
\text { mild, late }\end{array}$ \\
\hline & AGA & \multicolumn{2}{|l|}{ SGA } & AGA \\
\hline
\end{tabular}

$N G$, normal growth; SG, small growth; Nl, normal; Abnl, abnormal; MVM, maternal vascular malperfusion; FVM, fetal vascular malperfusion; AGA, appropriate-for-gestational age; SGA, small-for-gestational age.

The two phenotypes of FGR have similarities in most key characteristics with the main difference that FGR-I have more severe and early features. Fetuses of both FGR phenotypes fail to reach their growth potential, however, birthweight in FGR-I is frequently below the $3^{\text {rd }}$ centile, but in FGR-II can be within the reference range $\left(10^{\text {th }}-90^{\text {th }}\right.$ centile). Similarly, placental weight can follow the same pattern of difference between FGR-I and FGR-II. Placental histology and biomarkers will also be divergent reflecting either different mechanisms of placental dysfunction or a varying degrees of damage within the same spectrum of placental dysfunction. In addition, a clinically important distinction between FGR-I and FGR-II can be done based on Doppler examination of the fetoplacental circulation. FGR-I is a consequence of abnormal maternal spiral artery modification, documented, in proxy, by high resistance in uterine artery Doppler indices, as well early severe abnormalities in umbilical and fetal Doppler waveforms. FGR-II, instead, is only apparent by the extent of fetal hemodynamic adaptation to reduced placental oxygenation, best documented by a higher CPR. Angiogenic biomarkers are also 
differentially regulated in the two subtypes of FGR with FGR-I showing more prominent and early changes compared to FGR-II.

An important conclusion can be made: fetuses with reduced growth velocity are more at risk of adverse neonatal outcomes, and depending on the severity of the placental dysfunction, the clinical impact of growth impairment can be severe and early or late and unpredictable.

\section{Feasibility of a third trimester biometry scan}

In the consensus definition of fetal growth restriction, the parameter 'crossing centiles' indicates the need for at least one growth scan during the third trimester of pregnancy [5]. In chapter 2 of this thesis, a third trimester growth scan made it possible to calculate growth velocities between the second and third trimester, and detect fetuses with reduced growth velocity who are at risk of adverse outcome. The Dutch NVOG guideline does not recommend a routine third trimester scan in low-risk women; instead it only recommends this for women with an increased risk of FGR based on history or maternal characteristics [34]. In a review of McCowan, evaluating national guidelines of 6 countries, five of the six guidelines (83\%), did not recommend a routine third-trimester scan in lowrisk women [35]. The recommendations in these national guidelines are largely influenced by a meta-analysis of randomized trials included in a Cochrane review of routine ultrasound $>24$ weeks in low risk women ( 8 trials, $\mathrm{n}=27024$ women) performed the third-trimester scan too early ( $>24$ weeks) for optimum detection of late onset FGR [36]. Critical appraisal of the Cochrane review shows that most included studies were underpowered to detect an impact on stillbirth. A recently published nationwide stepped randomized trial in the Netherlands (IRIS study) in a low risk population demonstrated that routine ultrasonography in the third trimester (to detect low AC and EFW, not growth rate) was associated with higher antenatal detection of SGA fetuses $32 \%$ versus $19 \%$, but not with a reduced incidence of severe adverse perinatal outcomes compared with usual care alone [37]. In the IRIS study, an AC reduction of more than 20 centiles was used to define FGR. This criterium is probably not strict enough in a low-risk population, which can result in false positive labelled FGR cases with the risk of over-intervention. The findings of this study did not support routine ultrasonography in the third trimester for low risk pregnancies [38].

The POP-study, nulliparous women with mixed risk (low and high risk), demonstrated that a universal research scan at 36 weeks correctly identified $57 \%$ of women who delivered SGA babies, whereas selective use of third trimester scanning identified just $20 \%$ of SGA. This study showed the role of reduced growth 
velocity. SGA fetuses with reduced AC growth velocity between the second and third trimester scan had the highest morbidity [39].

The targeted population can influence the additional value of using a third trimester scan and measuring growth velocity. Healthy fetuses may be more likely to be falsely labelled as FGR in a low-risk population, compared with a high-risk population. These false positive results, can lead to iatrogenic maternal and fetal harm [40]. However, in a high risk population there will be more fetuses missed as FGR and falsely labeled as false negative, which can subsequently cause fetal harm and even stillbirth [41]. Future studies on the implementation of third trimester growth scan in clinical obstetric practice should focus on methodological design and selecting a target population with the highest gain from this intervention.

\section{Implementation of fetal growth velocity in clinical practice}

For the clinical implementation of a new, updated, and inclusive definition of FGR, some caveats of fetal growth velocity need to be addressed. The literature about longitudinal growth assessment for the prediction of adverse neonatal outcome is still inconsistent. This could be due to methodological differences in calculating growth velocities (AC or EFW, Z-velocity or individualized), the targeted population (low-risk vs high-risk) and the different outcome measurements [42]. Growth velocity is often calculated as the change in fetal size between two time points during gestation, e.g. the rate of fetal estimated fetal weight (EFW) or a specific biometric index (abdominal circumference $[\mathrm{AC}]$ ) over a given time interval (gram or mm/week) [43-47].

Despite using different reference curves and cut-off levels, several studies have shown the role of fetal growth velocities in identifying at-risk fetuses and improved clinical outcomes above a single measurement of fetal size. Normal fetal growth - defined as EFW growth between the $25^{\text {th }}-75^{\text {th }}$ centile - was shown to be protective for stillbirths, while growth trajectories slower than the $3^{\text {rd }}$ centile - regardless of whether they are SGA at birth - are associated with adverse perinatal outcome [44,45]. In a prospective cohort study of more than 4,000 singleton pregnant women, AGA fetuses with a fetal growth deceleration $>50^{\text {th }}$ percentile between the second and third trimester were at an increased risk for NICU admission (OR 1.8) and perinatal death (OR 3.8) [46]. Another study found that a decline of EFW percentile velocity $<50^{\text {th }}$ among controls have a 4.7 fold increased risk to die antepartum [47]. Accumulating evidence suggests that abnormal AC fetal growth velocity is associated with adverse neonatal outcomes in both SGA and LGA fetuses in universal screening [39, 48]. AC growth velocity is a risk factor for adverse neonatal outcomes which is independent of EFW [49]. The POP study - a recent prospective cohort study - in 3,977 nulliparous women with 
mixed risk (low and high risk) shows that an EFW of less than the $10^{\text {th }}$ centile was not associated with neonatal morbidity if the neonates AC growth velocity was normal, but was associated with a risk of neonatal morbidity approximately four times higher than if the AC growth velocity was in the lowest decile in addition to a worse neonatal outcome. SGA fetuses with reduced AC growth velocity (more than approximately a 40 centile drop between the 20- and 36-week scans), had the highest morbidity [39]. Another prospective study showed that a decline in growth velocity of more than 30 centiles in the third trimester was associated with an abnormal doppler (CPR) and more placental histological lesions [50]. In the absence of consensus on a clear cut-off value, we used in this thesis an easy and practical method of calculating fetal AC velocity between 20 and 32 weeks in $\mathrm{mm} /$ week and AC velocity less than $20^{\text {th }}$ centile to define reduced fetal growth. AGA fetuses with a reduced AC growth velocity, in a high-risk study population, were associated with more adverse neonatal outcomes compared with AGA fetuses with a normal AC growth velocity (chapter 2). These observations, in light of the existing literature, indicate that fetal growth velocity has a potential clinical utility for predicting adverse neonatal outcome, regardless of the methodology or cut-off value.

\section{The additional value of placental angiogenic markers}

The discovery of placental angiogenic biomarkers has led to great advancement in our understanding of placental dysfunction disorders, mainly preeclampsia and FGR $[24,51,52]$. In the last decades, the role of angiogenic biomarkers in predicting PE has been widely publicized resulting in validated and widely accepted cut-off levels that have found their way into clinical practice in many countries $[53,54]$. Around $70 \%$ of women with early FGR will develop hypertensive disorders, mainly preeclampsia [55], implying the common underlying mechanism for placental dysfunction. In chapter 5, we performed a systematic review of literature to define optimal gestational age-specific cut-off values for angiogenic biomarkers: sFlt-1, PlGF and sFlt-1/PIGF ratio for the prediction of FGR or SGA fetuses at risk for placental pathology and adverse outcomes. The included studies in our systematic review gave insight in longitudinal changes in these angiogenic serum levels during normal and SGA pregnancies. Data on similar changes in FGR pregnancies were scarce and heterogeneous, because of a wide variation in FGR definition, different study designs, and used assays. Because of the heterogeneity, it is not yet possible to define a cut-off value for sFlt-1, PlGF and sFlt-1/PlGF for FGR. However, the sFlt-1/PIGF ratio is potentially useful in the prediction of SGA pregnancies with underlying placental pathology in high-risk women. A recent prospective study of early-onset FGR demonstrated, that almost all pregnancies 
complicated by early-onset FGR without PE exceeded the cut-off value of 38 for sFlt-1/PIGF, proposed for the suspicion of PE, and 75\% exceeded the cut-off value of 85, proposed for the diagnosis of PE. These cut-off values are not necessarily specific for PE, but for placental dysfunction-related disorders in general and can be used in recognition of high-risk women [16, 56-58]. The sFlt-1/PlGF ratio is highly predictive for ruling out PE. Using these angiogenic biomarkers in routine management of PE may improve clinical care and avoid unnecessary hospitalization, which has a significant social and economic impact [59]. Therefore, we argue that assessment of placental angiogenic biomarkers, mainly sFlt-1/P1GF ratio may distinguish the fetus with placenta-mediated FGR (FGR type 1 and 2) from the constitutionally small (small-growing) fetus, thereby avoiding iatrogenic preterm delivery in the latter group.

\section{Gestational age-dependent model for prediction and management of FGR}

The identification of a FGR fetus during pregnancy is associated with improvement in neonatal outcomes, compared with the undetected FGR [60]. The reason for this improved outcome is probably the fact that detection of FGR and subsequent adequate antenatal monitoring leads to a more active management of labor and delivery [61]. Simple prediction models utilizing a single maternal clinical factor, such as maternal anthropometry demonstrate poor prediction accuracy [62]. Therefore, improved predictive performance can be expected if different maternal characteristics would be combined in multiparameter models $[63,64]$. The RCOG guideline 'The investigation and Management of the SGA Fetus' has defined minor and major risk factors in the maternal characteristics (at 12 weeks). Major risk factors include maternal age $>40$ years, smoking $>11$ cigarettes per day, cocaine use, daily vigorous exercise, previous SGA baby and previous stillbirth. If one major risk factor applies, a woman should prompt referral for serial ultrasound measurement of fetal size and assessment of wellbeing with Doppler [65]. Adding placental angiogenic biomarkers and ultrasound fetal biometry to these maternal characteristics can potentially further improve the prediction of FGR [66]. Besides the parameters included in the model, the predictive value and the clinical utility depend on the trimester of pregnancy in which the model is implemented. The main goal of a first trimester model is to identify the women at risk for severe early phenotype (FGR-I) and to enable early initiation of therapeutic intervention to alter the course the disease, as has been shown in the meta-analysis of nine trails on the use of aspirin (including 1317 women) [67], and a recent large international RCT (Aspre trial) [68]. Conversely, prediction models in the third trimester can help to optimize monitoring and 
timing of delivery in pregnancies with suspected late FGR-II [69-71]. Figure 2 shows a proposed model for screening and follow-up of suspected FGR in each trimester of pregnancy using maternal characteristics (MC), doppler, biometry and biomarker measurements. It depends on the screening results if a fetus is classified as low, medium or high risk.

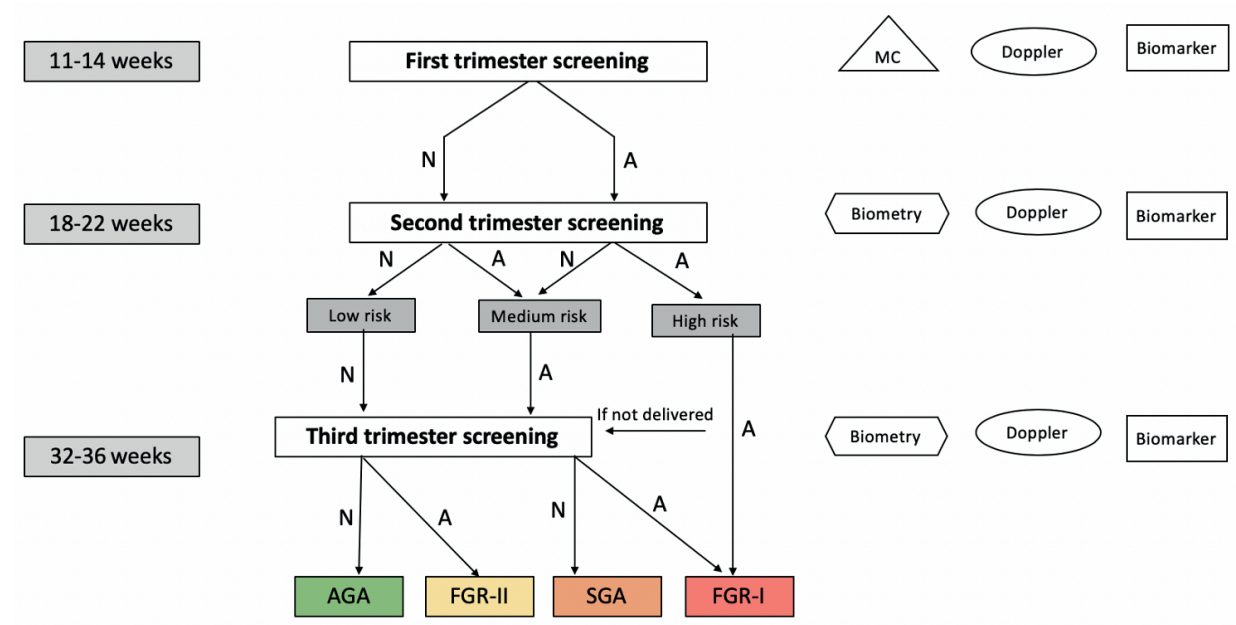

Figure 2 Systematic overview of multi-parameter models during three trimesters

$\mathrm{N}$, normal screening result; A, abnormal screening result; AGA, appropriate-for-gestational age; SGA, smallfor-gestational age; FGR, fetal growth restriction, type I and II; MC, maternal characteristics.

\section{First trimester prediction and monitoring of FGR}

Dysfunction of the placenta that leads to FGR-I has its pathophysiological roots in the early stages of placentation and can manifest from the end of the first trimester of pregnancy [13]. This process of early placental dysfunction could be identified using a combination of biophysical (maternal blood pressure and uterine artery doppler) and biochemical (placental angiogenic levels) markers in the first trimester [66, 72]. Doppler abnormalities of the uterine artery reflect early spiral artery maladaptation in the placental bed characteristic of PE and FGR, and may be present many weeks before severe cardiovascular and metabolic deterioration occurs. Chronic ischemia of the placental villi impairs secretion of the pro-angiogenic factor, e.g. PlGF, and leads to excessive release of anti-angiogenic factors, e.g. sFlt-1, by syncytial knots consequently resulting in elevated sFlt-1/ PIGF ratio in early onset FGR. Several studies have shown that the addition of placental biomarkers levels to maternal characteristics increased the detection rate for early and late SGA [73, 74]. 
The Fetal Medicine Foundation (FMF) developed a tool for estimating the risk of FGR, defined as a birth weight $<5^{\text {th }}$ centile for gestational age, using a combination of maternal characteristics, previous obstetric history, MAP, UtA-PI, PAPP-A and PlGF, at 11-13 weeks [73, 75]. Studies have demonstrated that this algorithm could potentially identify half of pregnancies that deliver SGA neonates in the absence of PE, at a $10 \%$ FPR. It is noteworthy that the outcome variable of the FMF predictive model was SGA instead of FGR, although the predictive performance was greater for the subgroup of SGA delivering before 37 weeks (73\%) than those delivering at or after 37 weeks (46\%). This is arguably because the proportion of FGR fetuses in the preterm SGA is likely to be higher than in the term SGA group. This suggests that the early biophysical and placental biomarkers described in the FMF model could be used for identifying the FGR subgroup amongst the SGA fetuses. In chapter 7, we validated the FMF model for term SGA and fetus with reduced fetal growth velocity in our population. At a $10 \%$ FPR, the sensitivity was $18.2 \%$ with only maternal characteristics and slightly improved to $23.4 \%$ with the addition of angiogenic and biophysical markers for term SGA. The sensitivity was $29.1 \%$ for reduced growth velocity as outcome measurement. Reduced fetal growth velocity was used as outcome measurement, suggesting a FGR type II. The addition of biomarkers and MAP to the baseline screening has less predictive value for term SGA, suggesting that a large part of the term SGA fetuses is normal small, without abnormal biomarker levels. This also highlights the problem of FGR type II; there are milder placental problems, not yet measurable in first trimester. Overall, the predicted probability was lower than the observed proportion of SGA fetuses and both models tended to underestimate the risk of term SGA and FGR type II in our population. The lower detection rates of SGA in other studies that attempted to validate the original FMF data could be due to variation in the a priori FGR risk across populations or measurement errors [76-78]. We were unable to use the complete FMF model because of the retrospective design as only in a small subgroup Uterine artery Pulsatility Index (UtPI) Doppler values were available. The UtPI measurement is not performed as part of routine care for the general obstetrical population. Only in high-risk pregnant women with check-ups in second-third level centers these UtPI measurements are performed. However, with the upcoming interest in the implementation of a routine 13 weeks scan for all pregnant women in the Netherlands (IMITAS-study), measuring the UtPI may become more commonplace in Dutch perinatal centers. In conclusion, a modern multiparameter first trimester screening for FGR is essential for risk selection and allocation of antenatal follow-up resources. 


\section{Second trimester prediction and monitoring of FGR}

In the second trimester of pregnancy, severe placental dysfunction can develop leading to a spectrum of obstetric complications. According to the RCOG guideline, relevant major risk factors during second trimester for SGA are PE, severe pregnancy induced hypertension, low maternal weight gain and low PAPP-A levels [65]. The second trimester scan, routinely carried out around 20 weeks' gestation has a role in evaluating fetal organs and structures for anomalies, as well as assessing fetal growth by measuring biometric parameters and calculating the estimated fetal weight relative to a reference population. Studies have described the use of these biometric measurements in multiparameter models to redefine pregnancies in low, medium or high risk (Figure 2) [79-81] . Consequently, high risk pregnancies that are thought to be at risk of placental insufficiency could be monitored with serial growth scans and Doppler measurements [82]. The use of the umbilical artery flow indices as a surveillance tool in these pregnancies has been shown to reduce perinatal morbidity and mortality [83].

\section{Third trimester prediction and monitoring of FGR}

During the third trimester, prediction models generally have better predictive ability due to being temporally closer to the onset of clinical disease. In a prospective population-based cohort among 7,677 pregnant women, the detection of fetuses at risk for adverse birth outcomes nearly doubled, with the implementation of third trimester fetal ultrasound scan, combined with maternal characteristics, compared with only second trimester ultrasound [70]. Prediction models can also be improved because of the composition of the model (addition of biomarkers and/or growth velocity). In chapter 4 , the baseline prediction model, including maternal age, conception method, smoking, previous SGA, previous SGA stillbirth, previous PE, maternal BMI, $\beta$-hCG, PAPP-A, and abdominal circumference ultrasound at 32 weeks of GA, had a detection rate of $41.4 \%$ for SGA. Adding growth velocities between 20 and 32 weeks to the baseline screening improved the DR of SGA to $56.5 \%$. Furthermore, the combination of baseline screening, angiogenic biomarkers (PlGF and sFlt-1), and growth velocities between second and third trimester, gave a detection rate of $60.9 \%$, at a $10 \%$ FPR. Monitoring of high risk pregnancies in the third trimester aims at personalizing the decision to delivery and balancing the risks and benefits of iatrogenic delivery and unpredictable stillbirth [55, 84]. A combination of biochemical (PlGF and sFlt-1) and biophysical (UmbA Doppler, cerebroplacental ratio and cardiotocogram) tools can be useful in setting up a suitable monitoring strategy depending on local resources and protocols [85]. 


\section{Long-term follow-up of FGR fetuses}

Neonates born as FGR are at risk for metabolic and cardiovascular disease later in life [86]. In the first years after birth, growth-restricted children can show catchup growth, even in height and/or weight [87]. This is considered as a process of compensatory accelerated growth after a period of poor intrauterine growth. Since Barker first proposed the 'thrifty phenotype hypothesis' suggesting that restricted fetal growth could represent an important contributor to the development origins of adult metabolic and cardiovascular disease, several studies have demonstrated associations between low birth weight and long-term risk factors [88]. Literature remains unclear on whether catch-up growth has more benefits or hazards in the long term. Short-term benefits associated with catch-up growth include a lower infection risk, fewer hospitalizations, neurodevelopmental advantages and a lower mortality [89-91]. On the other hand, catch-up growth may have adverse effects on long-term health. As an adaptation to undernutrition in fetal life, permanent metabolic and endocrine changes occur which would be beneficial if nutrition remained scarce after birth. If nutrition becomes plentiful, however, these changes predispose to obesity and impaired glucose tolerance [92-94], resulting in the metabolic syndrome (high blood pressure, dyslipidemia, insulin resistance), and cardiovascular disease [95-99]. In our study, described in chapter 8, fetuses with FGR, based on reduced growth velocity, were at a significant risk of developing catch-up growth in height during first three years after birth. The Generation R study and CASyMIR cohort also found that a decreased antenatal growth velocity (without distinguishing between SGA and AGA) was associated with catch-up growth at two years of age $[41,100]$. Another recent study, focused on AGA fetuses with reduced fetal growth velocity, showed a catch-up growth in weight and length between birth and first 4 months [87]. AGA fetuses who experience reduced growth velocity during pregnancy, exhibit catch-up growth after birth. These findings suggest that these infants may also be at increased risk of developing postnatal health problems, and that increased awareness of clinicians is needed on the possible long-term consequences of FGR. 


\section{REFERENCES}

1. Larsen T, Greisen G, Petersen S. Intrauterine growth correlation to postnatal growthinfluence of risk factors and complications in pregnancy. Early Hum Dev. 1997;47(2):15765.

2. Deter RL, Lee W, Yeo L, Erez O, Ramamurthy U, Naik M, et al. Individualized growth assessment: conceptual framework and practical implementation for the evaluation of fetal growth and neonatal growth outcome. Am J Obstet Gynecol. 2018;218(2S):S656-S78.

3. Figueras F, Gratacos E. An integrated approach to fetal growth restriction. Best Pract Res Clin Obstet Gynaecol. 2017;38:48-58.

4. Figueras F, Gardosi J. Intrauterine growth restriction: new concepts in antenatal surveillance, diagnosis, and management. Am J Obstet Gynecol. 2011;204(4):288-300.

5. Gordijn SJ, Beune IM, Thilaganathan B, Papageorghiou A, Baschat AA, Baker PN, et al. Consensus definition of fetal growth restriction: a Delphi procedure. Ultrasound Obstet Gynecol. 2016;48(3):333-9.

6. Gaccioli F, Sovio U, Cook E, Hund M, Charnock-Jones DS, Smith GCS. Screening for fetal growth restriction using ultrasound and the sFLT1/PIGF ratio in nulliparous women: a prospective cohort study. Lancet Child Adolesc Health. 2018;2(8):569-81.

7. Fernandez-Gonzalez S, Ortiz-Arrabal O, Torrecillas A, Perez-Cruz M, Chueca N, GomezRoig MD, et al. Study of the fetal and maternal microbiota in pregnant women with intrauterine growth restriction and its relationship with inflammatory biomarkers: A case-control study protocol (SPIRIT compliant). Medicine (Baltimore). 2020;99(46):e22722.

8. Malhotra A, Allison BJ, Castillo-Melendez M, Jenkin G, Polglase GR, Miller SL. Neonatal Morbidities of Fetal Growth Restriction: Pathophysiology and Impact. Front Endocrinol (Lausanne). 2019;10:55.

9. Savchev S, Figueras F, Sanz-Cortes M, Cruz-Lemini M, Triunfo S, Botet F, et al. Evaluation of an optimal gestational age cut-off for the definition of early- and late-onset fetal growth restriction. Fetal Diagn Ther. 2014;36(2):99-105.

10. Figueras F, Gratacos E. Update on the diagnosis and classification of fetal growth restriction and proposal of a stage-based management protocol. Fetal Diagn Ther. 2014;36(2):8698.

11. Morales-Rosello J, Khalil A, Morlando M, Papageorghiou A, Bhide A, Thilaganathan B. Changes in fetal Doppler indices as a marker of failure to reach growth potential at term. Ultrasound Obstet Gynecol. 2014;43(3):303-10.

12. Oros D, Figueras F, Cruz-Martinez R, Meler E, Munmany M, Gratacos E. Longitudinal changes in uterine, umbilical and fetal cerebral Doppler indices in late-onset small-forgestational age fetuses. Ultrasound Obstet Gynecol. 2011;37(2):191-5.

13. Burton GJ, Jauniaux E. Pathophysiology of placental-derived fetal growth restriction. Am J Obstet Gynecol. 2018;218(2S):S745-S61.

14. Moraitis AA, Wood AM, Fleming M, Smith GC. Birth weight percentile and the risk of term perinatal death. Obstet Gynecol. 2014;124(2 Pt 1):274-83.

15. Vasak B, Koenen SV, Koster MP, Hukkelhoven CW, Franx A, Hanson MA, et al. Human fetal growth is constrained below optimal for perinatal survival. Ultrasound Obstet Gynecol. 2015;45(2):162-7. 
16. Ganer Herman H, Barber E, Gasnier R, Gindes L, Bar J, Schreiber L, et al. Placental pathology and neonatal outcome in small for gestational age pregnancies with and without abnormal umbilical artery Doppler flow. Eur J Obstet Gynecol Reprod Biol. 2018;222:52-6.

17. Salavati N, Smies M, Ganzevoort W, Charles AK, Erwich JJ, Plosch T, et al. The Possible Role of Placental Morphometry in the Detection of Fetal Growth Restriction. Front Physiol. 2018;9:1884.

18. Crovetto F, Triunfo S, Crispi F, Rodriguez-Sureda V, Roma E, Dominguez C, et al. Firsttrimester screening with specific algorithms for early- and late-onset fetal growth restriction. Ultrasound Obstet Gynecol. 2016;48(3):340-8.

19. Spinillo A, Gardella B, Bariselli S, Alfei A, Silini E, Dal Bello B. Placental histopathological correlates of umbilical artery Doppler velocimetry in pregnancies complicated by fetal growth restriction. Prenat Diagn. 2012;32(13):1263-72.

20. Erez O, Romero R, Espinoza J, Fu W, Todem D, Kusanovic JP, et al. The change in concentrations of angiogenic and anti-angiogenic factors in maternal plasma between the first and second trimesters in risk assessment for the subsequent development of preeclampsia and small-for-gestational age. J Matern Fetal Neonatal Med. 2008;21(5):279-87.

21. Rana S, Karumanchi SA, Levine RJ, Venkatesha S, Rauh-Hain JA, Tamez H, et al. Sequential changes in antiangiogenic factors in early pregnancy and risk of developing preeclampsia. Hypertension. 2007;50(1):137-42.

22. Kingdom JC, Audette MC, Hobson SR, Windrim RC, Morgen E. A placenta clinic approach to the diagnosis and management of fetal growth restriction. Am J Obstet Gynecol. 2018;218(2S):S803-S17.

23. Gibbs I, Leavey K, Benton SJ, Grynspan D, Bainbridge SA, Cox BJ. Placental transcriptional and histologic subtypes of normotensive fetal growth restriction are comparable to preeclampsia. Am J Obstet Gynecol. 2019;220(1):110 e1- e21.

24. Benton SJ, McCowan LM, Heazell AE, Grynspan D, Hutcheon JA, Senger C, et al. Placental growth factor as a marker of fetal growth restriction caused by placental dysfunction. Placenta. 2016;42:1-8.

25. Whitten AE, Romero R, Korzeniewski SJ, Tarca AL, Schwartz AG, Yeo L, et al. Evidence of an imbalance of angiogenic/antiangiogenic factors in massive perivillous fibrin deposition (maternal floor infarction): a placental lesion associated with recurrent miscarriage and fetal death. Am J Obstet Gynecol. 2013;208(4):310 e1- e11.

26. Miranda J, Triunfo S, Rodriguez-Lopez M, Sairanen M, Kouru H, Parra-Saavedra M, et al. Performance of third-trimester combined screening model for prediction of adverse perinatal outcome. Ultrasound Obstet Gynecol. 2017;50(3):353-60.

27. Baschat AA, Cosmi E, Bilardo CM, Wolf H, Berg C, Rigano S, et al. Predictors of neonatal outcome in early-onset placental dysfunction. Obstet Gynecol. 2007;109(2 Pt 1):253-61.

28. Boers KE, Vijgen SM, Bijlenga D, van der Post JA, Bekedam DJ, Kwee A, et al. Induction versus expectant monitoring for intrauterine growth restriction at term: randomised equivalence trial (DIGITAT). BMJ. 2010;341:c7087.

29. Baschat AA, Gembruch U. The cerebroplacental Doppler ratio revisited. Ultrasound Obstet Gynecol. 2003;21(2):124-7.

30. Kovo M, Schreiber L, Ben-Haroush A, Cohen G, Weiner E, Golan A, et al. The placental factor in early- and late-onset normotensive fetal growth restriction. Placenta. 2013;34(4):320-4.

31. Mifsud W, Sebire NJ. Placental pathology in early-onset and late-onset fetal growth restriction. Fetal Diagn Ther. 2014;36(2):117-28. 
32. Meher S, Hernandez-Andrade E, Basheer SN, Lees C. Impact of cerebral redistribution on neurodevelopmental outcome in small-for-gestational-age or growth-restricted babies: a systematic review. Ultrasound Obstet Gynecol. 2015;46(4):398-404.

33. Veglia M, Cavallaro A, Papageorghiou A, Black R, Impey L. Small-for-gestational-age babies after 37 weeks: impact study of risk-stratification protocol. Ultrasound Obstet Gynecol. 2018;52(1):66-71.

34. NVOG. Richtlijn Foetale groeirestrictie (FGR). 2017.

35. McCowan LM, Figueras F, Anderson NH. Evidence-based national guidelines for the management of suspected fetal growth restriction: comparison, consensus, and controversy. Am J Obstet Gynecol. 2018;218(2S):S855-S68.

36. Bricker L, Medley N, Pratt JJ. Routine ultrasound in late pregnancy (after 24 weeks' gestation). Cochrane Database Syst Rev. 2015(6):CD001451.

37. Henrichs J, Verfaille V, Jellema P, Viester L, Pajkrt E, Wilschut J, et al. Effectiveness of routine third trimester ultrasonography to reduce adverse perinatal outcomes in low risk pregnancy (the IRIS study): nationwide, pragmatic, multicentre, stepped wedge cluster randomised trial. BMJ. 2019;367:15517.

38. Napolitano. Routine third trimester ultrasonography in low risk pregnancy (the IRIS study): Not all is lost. BMJ. 2020.

39. Sovio U, White IR, Dacey A, Pasupathy D, Smith GCS. Screening for fetal growth restriction with universal third trimester ultrasonography in nulliparous women in the Pregnancy Outcome Prediction (POP) study: a prospective cohort study. Lancet. 2015;386(10008):208997.

40. Monier I, Blondel B, Ego A, Kaminiski M, Goffinet F, Zeitlin J. Poor effectiveness of antenatal detection of fetal growth restriction and consequences for obstetric management and neonatal outcomes: a French national study. BJOG. 2015;122(4):518-27.

41. Broere-Brown ZA, Schalekamp-Timmermans S, Jaddoe VWV, Steegers EAP. Deceleration of fetal growth rate as alternative predictor for childhood outcomes: a birth cohort study. BMC Pregnancy Childbirth. 2019;19(1):216.

42. Hadlock FP, Harrist RB, Sharman RS, Deter RL, Park SK. Estimation of fetal weight with the use of head, body, and femur measurements-a prospective study. Am J Obstet Gynecol. 1985;151(3):333-7.

43. Grantz KL, Kim S, Grobman WA, Newman R, Owen J, Skupski D, et al. Fetal growth velocity: the NICHD fetal growth studies. Am J Obstet Gynecol. 2018;219(3):285 e1- e36.

44. Francis AH, O; Gardosi, J. Slow growth defined by customised growth trajectory and adverse perinatal outcome. International Conference of Fetal Growth. 2019.

45. Iliodromiti S, Mackay DF, Smith GC, Pell JP, Sattar N, Lawlor DA, et al. Customised and Noncustomised Birth Weight Centiles and Prediction of Stillbirth and Infant Mortality and Morbidity: A Cohort Study of 979,912 Term Singleton Pregnancies in Scotland. PLoS Med. 2017;14(1):e1002228.

46. Chatzakis C, Papaioannou GK, Eleftheriades M, Makrydimas G, Dinas K, Sotiriadis A. Perinatal outcome of appropriate-weight fetuses with decelerating growth. J Matern Fetal Neonatal Med. 2019:1-8.

47. Pacora P, Romero R, Jung E, Gudicha DW, Hernandez-Andrade E, Musilova I, et al. Reduced fetal growth velocity precedes antepartum fetal death. Ultrasound Obstet Gynecol. 2020. 
48. Sovio U, Moraitis AA, Wong HS, Smith GCS. Universal vs selective ultrasonography to screen for large-for-gestational-age infants and associated morbidity. Ultrasound Obstet Gynecol. 2018;51(6):783-91.

49. Cavallaro A, Veglia M, Svirko E, Vannuccini S, Volpe G, Impey L. Using fetal abdominal circumference growth velocity in the prediction of adverse outcome in near-term smallfor-gestational-age fetuses. Ultrasound Obstet Gynecol. 2018;52(4):494-500.

50. MacDonald TM, Hui L, Tong S, Robinson AJ, Dane KM, Middleton AL, et al. Reduced growth velocity across the third trimester is associated with placental insufficiency in fetuses born at a normal birthweight: a prospective cohort study. BMC Med. 2017;15(1):164 .

51. Maynard SE, Karumanchi SA. Angiogenic factors and preeclampsia. Semin Nephrol. 2011;31(1):33-46.

52. Vrachnis N, Kalampokas E, Sifakis S, Vitoratos N, Kalampokas T, Botsis D, et al. Placental growth factor (P1GF): a key to optimizing fetal growth. J Matern Fetal Neonatal Med. 2013;26(10):995-1002.

53. Agrawal S, Cerdeira AS, Redman C, Vatish M. Meta-Analysis and Systematic Review to Assess the Role of Soluble FMS-Like Tyrosine Kinase-1 and Placenta Growth Factor Ratio in Prediction of Preeclampsia: The SaPPPhirE Study. Hypertension. 2018;71(2):306-16.

54. Dragan I, Georgiou T, Prodan N, Akolekar R, Nicolaides KH. Screening for pre-eclampsia using sFlt-1/PlGF ratio cut-off of 38 at 30-37 weeks' gestation. Ultrasound Obstet Gynecol. 2017;49(1):73-7.

55. Lees C, Marlow N, Arabin B, Bilardo CM, Brezinka C, Derks JB, et al. Perinatal morbidity and mortality in early-onset fetal growth restriction: cohort outcomes of the trial of randomized umbilical and fetal flow in Europe (TRUFFLE). Ultrasound Obstet Gynecol. 2013;42(4):400-8.

56. Herraiz I, Quezada MS, Rodriguez-Calvo J, Gomez-Montes E, Villalain C, Galindo A. Longitudinal change of sFlt-1/PIGF ratio in singleton pregnancy with early-onset fetal growth restriction. Ultrasound Obstet Gynecol. 2018;52(5):631-8.

57. MacDonald TM, Tran C, Kaitu'u-Lino TJ, Brennecke SP, Hiscock RJ, Hui L, et al. Assessing the sensitivity of placental growth factor and soluble fms-like tyrosine kinase 1 at 36 weeks' gestation to predict small-for-gestational-age infants or late-onset preeclampsia: a prospective nested case-control study. BMC Pregnancy Childbirth. 2018;18(1):354.

58. Baekgaard Thorsen LH, Bjorkholt Andersen L, Birukov A, Lykkedegn S, Dechend R, Stener Jorgensen J, et al. Prediction of birth weight small for gestational age with and without preeclampsia by angiogenic markers: an Odense Child Cohort study. J Matern Fetal Neonatal Med. 2020;33(8):1377-84.

59. Caillon H, Tardif C, Dumontet E, Winer N, Masson D. Evaluation of sFlt-1/PlGF Ratio for Predicting and Improving Clinical Management of Pre-eclampsia: Experience in a Specialized Perinatal Care Center. Ann Lab Med. 2018;38(2):95-101.

60. Voskamp BJ, Beemsterboer DH, Verhoeven CJ, Oude Rengerink K, Ravelli AC, Bakker JJ, et al. Potential improvement of pregnancy outcome through prenatal small for gestational age detection. [correction]. Am J Perinatol. 2014;31(12):1093-104.

61. Pattinson R, Kerber K, Buchmann E, Friberg IK, Belizan M, Lansky S, et al. Stillbirths: how can health systems deliver for mothers and babies? Lancet. 2011;377(9777):1610-23.

62. Goto E. Maternal anthropometry to predict small for gestational age: a meta-analysis. Eur J Obstet Gynecol Reprod Biol. 2016;203:193-8. 
63. Leite DFB, Cecatti JG. Fetal Growth Restriction Prediction: How to Move beyond. ScientificWorldJournal. 2019;2019:1519048.

64. van Montfort P, Scheepers HCJ, Dirksen CD, van Dooren IMA, van Kuijk SMJ, Meertens LJE, et al. Impact on perinatal health and cost-effectiveness of risk-based care in obstetrics: a before after study. Am J Obstet Gynecol. 2020.

65. RCOG. The investigation and management of the small-for-gestational-age fetus. 2013. Available from: https://www.rcog.org.uk/globalassets/documents/guidelines/gtg_31.pdf.

66. Gaccioli F, Aye I, Sovio U, Charnock-Jones DS, Smith GCS. Screening for fetal growth restriction using fetal biometry combined with maternal biomarkers. Am J Obstet Gynecol. 2018;218(2S):S725-S37.

67. Bujold E, Morency AM, Roberge S, Lacasse Y, Forest JC, Giguere Y. Acetylsalicylic acid for the prevention of preeclampsia and intra-uterine growth restriction in women with abnormal uterine artery Doppler: a systematic review and meta-analysis. J Obstet Gynaecol Can. 2009;31(9):818-26.

68. Rolnik DL, Wright D, Poon LC, O'Gorman N, Syngelaki A, de Paco Matallana C, et al. Aspirin versus Placebo in Pregnancies at High Risk for Preterm Preeclampsia. N Engl J Med. 2017;377(7):613-22.

69. Lesmes C, Gallo DM, Gonzalez R, Poon LC, Nicolaides KH. Prediction of small-for-gestational-age neonates: screening by maternal serum biochemical markers at 19-24 weeks. Ultrasound Obstet Gynecol. 2015;46(3):341-9.

70. Erkamp JS, Voerman E, Steegers EAP, Mulders A, Reiss IKM, Duijts L, et al. Second and third trimester fetal ultrasound population screening for risks of preterm birth and small-size and large-size for gestational age at birth: a population-based prospective cohort study. BMC Med. 2020;18(1):63.

71. Valino N, Giunta G, Gallo DM, Akolekar R, Nicolaides KH. Biophysical and biochemical markers at 35-37 weeks' gestation in the prediction of adverse perinatal outcome. Ultrasound Obstet Gynecol. 2016;47(2):203-9.

72. Smith GC. First-trimester determination of complications of late pregnancy. JAMA. 2010;303(6):561-2.

73. Karagiannis G, Akolekar R, Sarquis R, Wright D, Nicolaides KH. Prediction of small-forgestation neonates from biophysical and biochemical markers at 11-13 weeks. Fetal Diagn Ther. 2011;29(2):148-54.

74. Crovetto F, Triunfo S, Crispi F, Rodriguez-Sureda V, Dominguez C, Figueras F, et al. Differential performance of first-trimester screening in predicting small-for-gestational-age neonate or fetal growth restriction. Ultrasound Obstet Gynecol. 2017;49(3):349-56.

75. Poon LC, Syngelaki A, Akolekar R, Lai J, Nicolaides KH. Combined screening for preeclampsia and small for gestational age at 11-13 weeks. Fetal Diagn Ther. 2013;33(1):16-27.

76. Mosimann B, Pfiffner C, Amylidi-Mohr S, Risch L, Surbek D, Raio L. First trimester combined screening for preeclampsia and small for gestational age - a single centre experience and validation of the FMF screening algorithm. Swiss Med Wkly. 2017;147:w14498.

77. Rezende L, Rezende K, Guimaraes M, Dourado AL, da Matta F, Amim Junior J, et al. Evaluation of fetal medicine foundation algorithm in predictingsmall-for-gestational-age neonates. J Matern Fetal Neonatal Med. 2019:1-7.

78. Graham K, Park F, McLennan A, Pelosi M, Williams P, Poon LC, et al. Clinical evaluation of a first trimester pregnancy algorithm predicting the risk of small for gestational age neonates. Aust N Z J Obstet Gynaecol. 2019;59(5):670-6. 
79. Caradeux J, Martinez-Portilla RJ, Peguero A, Sotiriadis A, Figueras F. Diagnostic performance of third-trimester ultrasound for the prediction of late-onset fetal growth restriction: a systematic review and meta-analysis. Am J Obstet Gynecol. 2019;220(5):449-59 e19.

80. Akolekar R, Panaitescu AM, Ciobanu A, Syngelaki A, Nicolaides KH. Two-stage approach for prediction of small-for-gestational-age neonate and adverse perinatal outcome by routine ultrasound examination at 35-37 weeks' gestation. Ultrasound Obstet Gynecol. 2019;54(4):484-91.

81. Dall'Asta A, Brunelli V, Prefumo F, Frusca T, Lees CC. Early onset fetal growth restriction. Matern Health Neonatol Perinatol. 2017;3:2.

82. Alfirevic Z, Stampalija T, Gyte GM. Fetal and umbilical Doppler ultrasound in high-risk pregnancies. Cochrane Database Syst Rev. 2010(1):CD007529.

83. Morris RK, Malin G, Robson SC, Kleijnen J, Zamora J, Khan KS. Fetal umbilical artery Doppler to predict compromise of fetal/neonatal wellbeing in a high-risk population: systematic review and bivariate meta-analysis. Ultrasound Obstet Gynecol. 2011;37(2):135-42.

84. Sharp A, Jackson R, Cornforth C, Harrold J, Turner MA, Kenny L, et al. A prediction model for short-term neonatal outcomes in severe early-onset fetal growth restriction. Eur J Obstet Gynecol Reprod Biol. 2019;241:109-18.

85. Figueras F, Gratacos E. Stage-based approach to the management of fetal growth restriction. Prenat Diagn. 2014;34(7):655-9.

86. Salam RA, Das JK, Bhutta ZA. Impact of intrauterine growth restriction on long-term health. Curr Opin Clin Nutr Metab Care. 2014;17(3):249-54.

87. McLaughlin EJ, Hiscock RJ, Robinson AJ, Hui L, Tong S, Dane KM, et al. Appropriate-forgestational-age infants who exhibit reduced antenatal growth velocity display postnatal catch-up growth. PLoS One. 2020;15(9):e0238700.

88. Barker DJ, Winter PD, Osmond C, Margetts B, Simmonds SJ. Weight in infancy and death from ischaemic heart disease. Lancet. 1989;2(8663):577-80.

89. Victora CG, Barros FC, Horta BL, Martorell R. Short-term benefits of catch-up growth for small-for-gestational-age infants. Int J Epidemiol. 2001;30(6):1325-30.

90. Ong CY, Liao AW, Spencer K, Munim S, Nicolaides KH. First trimester maternal serum free beta human chorionic gonadotrophin and pregnancy associated plasma protein A as predictors of pregnancy complications. BJOG. 2000;107(10):1265-70.

91. Ong KK. Catch-up growth in small for gestational age babies: good or bad? Curr Opin Endocrinol Diabetes Obes. 2007;14(1):30-4.

92. Vorherr H. Factors influencing fetal growth. Am J Obstet Gynecol. 1982;142(5):577-88.

93. Bloomfield FH, Jaquiery AL, Oliver MH. Nutritional regulation of fetal growth. Nestle Nutr Inst Workshop Ser. 2013;74:79-89.

94. Touwslager RN, Houben AJ, Tan FE, Gielen M, Zeegers MP, Stehouwer CD, et al. Growth and endothelial function in the first 2 years of life. J Pediatr. 2015;166(3):666-71 e1.

95. Singhal A. Should We Promote Catch-Up Growth or Growth Acceleration in Low-Birthweight Infants? Nestle Nutr Inst Workshop Ser. 2015;81:51-60.

96. Singhal A. name name-style=" western" $>$ Cole TJ $<$ name name-style=" western" $>$ Fewtrell $\mathrm{M}$, et al: Promotion of faster weight gain in infants born small for gestational age is there an adverse effect on later blood pressure. Circulation. 2007;115:213-20.

97. Singhal A, Kennedy K, Lanigan J, Fewtrell M, Cole TJ, Stephenson T, et al. Nutrition in infancy and long-term risk of obesity: evidence from 2 randomized controlled trials. The American journal of clinical nutrition. 2010;92(5):1133-44. 
98. Weber M, Grote V, Closa-Monasterolo R, Escribano J, Langhendries J-P, Dain E, et al. Lower protein content in infant formula reduces BMI and obesity risk at school age: follow-up of a randomized trial. The American journal of clinical nutrition. 2014;99(5):1041-51.

99. Inostroza J, Haschke F, Steenhout P, Grathwohl D, Nelson SE, Ziegler EE. Low-protein formula slows weight gain in infants of overweight mothers. Journal of pediatric gastroenterology and nutrition. 2014;59(1):70.

100. Beltrand J, Nicolescu R, Kaguelidou F, Verkauskiene R, Sibony O, Chevenne D, et al. Catchup growth following fetal growth restriction promotes rapid restoration of fat mass but without metabolic consequences at one year of age. PLoS One. 2009;4(4):e5343. 
c) 


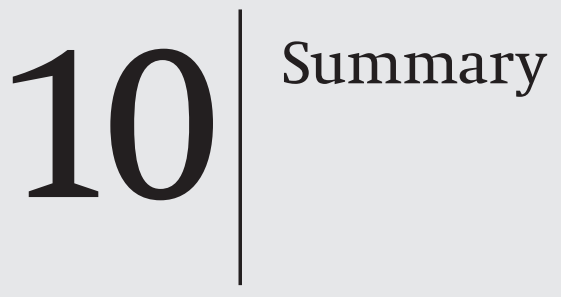



This thesis provides insight into the role of fetal growth velocity and placental biomarkers in identifying fetus with growth restriction during pregnancy and the association with neonatal outcomes. This chapter summarizes the findings of the research performed in this thesis and highlights the most important conclusions and implications for clinical practice and future research.

Chapter 1 presents a general introduction with the objectives of this thesis. This chapter describes the challenge of defining fetal growth restriction and the difference with small-for-gestational age (SGA) neonates. We introduce the concept of undetected fetal growth restriction in the group of appropriate-for-gestational age (AGA) neonates who are at increased risk of adverse neonatal outcome. We put forward the hypothesis that fetal growth velocities and placental biomarkers have a potential role in identifying these growth restricted fetuses.

Chapter 2 describes the association between reduced fetal growth velocities and adverse neonatal outcome in AGA neonates in a large retrospective cohort study. Growth velocities of the abdominal circumference, biparietal diameter, head circumference and femur length, were measured between 20 and 32 weeks of pregnancy. Neonates with a birth weight percentile between the $10-50^{\text {th }}$ had lower growth velocities compared to neonates with a birth weight between the $50^{\text {th }}-80^{\text {th }}$ percentile. Neonates with adverse neonatal outcome had significantly lower growth velocities of the abdominal circumference, head circumference and biparietal diameter. A reduced abdominal circumference growth velocity is associated with adverse neonatal outcome. Neonates with a birthweight lower than was expected earlier in pregnancy, based on the abdominal circumference measurement at 20 weeks, had more adverse neonatal outcomes, hospital and NICU stays at birth. This chapter highlights the importance of performing a third trimester scan to calculate growth velocities.

Chapter 3 evaluates the value of adding fetal growth velocity and first trimester maternal biomarkers to a baseline model for the prediction of SGA neonates and AGA neonates with reduced growth. The baseline model consists of maternal characteristics, obstetric history, $\beta$-hCG, PAPP-A and a single abdominal circumference measurement at 32 weeks. SGA neonates compared with AGA neonates had higher levels of sFlt-1 and a higher sFlt-1/PIGF ratio in first trimester. SGA neonates with reduced growth showed lower PAPP-A and $\beta$-hCG, higher sFlt- 1 and higher sFlt-1/PIGF ratio compared with AGA neonates with normal growth. AGA neonates with reduced growth showed lower PAPP-A levels and more adverse neonatal outcomes compared with AGA neonates with normal growth.

The addition of growth velocities or biomarkers to the baseline model improved the prediction of SGA neonates. The combination of baseline characteristics, growth velocity and placental biomarkers gave the best prediction for SGA. In 
conclusion, adding fetal growth velocity and placental biomarkers to baseline characteristics, improves the prediction of SGA neonates. Combining these predictors may recognize AGA neonates with reduced growth velocity and those at risk for adverse neonatal outcome.

Chapter 4 shows the longitudinal changes of biomarkers in women with early and late-onset placental dysfunction. The longitudinal changes in biomarkers compared with a single time point measurement are described. Early-onset placental dysfunction was defined as placenta-mediated complications (fetal growth restriction, preeclampsia and placental abruption) diagnosed before 34 weeks of gestation. Late-onset placental dysfunction was defined as preeclampsia or pregnancy induced hypertension, diagnosed after 34 weeks of gestation. Women with early-onset placental dysfunction had a lower increase in PIGF and a higher increase of sFlt-1 between 12 and 30 weeks of pregnancy compared with the reference group without placental dysfunction. The late-onset group did not show any differences in longitudinal biomarker levels compared with the reference group. During second trimester the sFlt-1/PIGF ratio had a better performance for the prediction of early-onset placental dysfunction than either sFlt-1 or PlGF alone. While during third trimester all individual biomarkers were significantly related to early-onset placental dysfunction. From first trimester, the longitudinal change of sFlt-1/PlGF ratio is already discriminatory for early-onset placental dysfunction. In conclusion, sFlt-1/PlGF ratio has the best predictive performance for early-onset placental dysfunction. Longitudinal measurements are only useful for early-onset placental disease. Late-onset placental dysfunction is a heterogeneous disease, and first trimester placental biomarkers have a poor predictive performance for identifying patients high-risk for developing a late-onset placental disease.

In Chapter 5 we conducted a systematic review including 26 studies to describe longitudinal changes in biomarker concentrations and optimal discriminatory cut-off values for PlGF and sFlt-1 in the screening of SGA neonates. There was a wide variety in study design, outcome definition, assay methods and time of screening, which makes the studies very heterogeneous. Using gestational age specific intervals, we showed that the largest differences between in the SGA and reference group were found in PlGF and after 26 weeks of gestational age. A moderate predictive performance was found with a wide range, reflecting the heterogeneity in studies. Because of the limited number of studies using fetal growth restriction as outcome, it was not possible to report optimal cut-off biomarker levels for this outcome. More research is needed to see if these biomarkers are sufficiently able to differentiate growth restriction on their own and how these biomarkers in combination with other relevant clinical and ultrasound parameters can be used in clinical routine diagnostics. 
Chapter 6 investigates the presence of maternal vascular malperfusion (MVM) lesions in SGA and subgroups of AGA neonates. MVM lesions are a consequence of defective remodeling of maternal spiral arteries, leading to fetal growth restriction. There is limited literature about the prevalence and impact of MVM lesions in pregnancies with normal fetal growth. Our study showed that MVM lesions are also encountered in placentas from AGA neonates. The presence of MVM lesions correlated inversely with birthweight even in AGA neonates. The combination of maternal characteristics, fetal growth velocity and placental biomarkers gave the best prediction for the presence of MVM lesions. These results suggest that a subgroup of normal weight neonates have features of FGR and are potentially at risk for adverse neonatal outcomes. Fetal growth velocity, placental biomarkers and the presence of MVM lesions may identify these high-risk fetuses.

Chapter 7 describes the validation of the fetal medicine foundation (FMF) first trimester risk predicting model for SGA in our own cohort. We used the model to calculate risks for term SGA and fetuses with reduced growth velocity using baseline screening and biomarkers. The performance of the FMF algorithm for term SGA was similar using either the baseline screening or the baseline screening combined with placental biomarkers and maternal mean arterial blood pressure. Models were poorly calibrated and tended to underestimate the risk of SGA and reduced growth velocity in our study population.

Chapter $\mathbf{8}$ shows postnatal growth measurements during the first five years of life in SGA and AGA neonates. Neonates born in the Maastricht University Medical Centre had check-ups at the Community Health Service (GGD). SGA neonates had a lower height and weight compared to AGA neonates till 3 years. Catch-up, defined as the difference in growth between 4 weeks and 3 years, was not different between both groups. However, neonates with reduced fetal growth had a higher risk for catch-up growth in height compared with normal growing fetuses. This suggests a role for fetal growth velocity in predicting fetal and even subsequent postnatal growth and consequently health risks in adulthood.

Chapter 9 elaborates on general discussion points of findings addresses in this manuscript within the context of recent literature. Subsequently, a reflection on the definition of fetal growth restriction is described, four conceptual phenotypes of fetal growth patterns are discussed, based on data presented in this thesis, combined with current knowledge about angiogenic biomarkers and placental histology. Practical implications about prediction and monitoring of fetal growth restriction and future perspectives were provided. 
c) 


$$
11 \text { Nederlandstalige samenvatting }
$$



Deze thesis verschaft inzicht in de rol van foetale groei snelheid en placentaire biomarkers in het identificeren van een foetus met groei vertraging tijdens de zwangerschap en de associatie met neonatale uitkomsten. Dit hoofdstuk vat de bevindingen samen van het onderzoek uitgevoerd in deze thesis en benadrukt de meest belangrijke conclusies en implicaties voor de klinische praktijk en toekomstig onderzoek.

Hoofdstuk 1 geeft een algemene introductie met de doelstellingen van dit proefschrift weer. Dit hoofdstuk beschrijft de uitdaging om foetale groei restrictie te definiëren en hoe het verschil met small-for-gestational age (SGA) neonaten te maken. We introduceren het concept van ongedetecteerde foetale groei vertraging in de groep van appropriate-for-gestational age (AGA) neonaten met een verhoogde kans op een afwijkende neonatale uitkomst. We hebben de hypothese naar voren gebracht dat foetale groei snelheden en placentaire biomarkers een potentiele rol hebben in het identificeren van deze groei vertraagde foetussen.

Hoofdstuk 2 beschrijft de associatie tussen verminderde groei snelheid en een afwijkende neonatale uitkomst in AGA neonaten in een grote retrospectieve cohort studie. Groeisnelheden van de buik omtrek, biparietale diameter, hoofdomtrek en femur lengte, werden bepaald tussen 20 en 32 weken van de zwangerschap. Neonaten met een geboorte gewicht tussen het $10-50^{\text {ste }}$ percentiel hadden lagere groei snelheden vergeleken met neonaten met een geboorte gewicht percentiel tussen $50-80^{\text {ste }}$ percentiel. Kinderen met een afwijkende neonatale uitkomst hadden een significant lagere groeisnelheid van de buik omtrek, hoofd omtrek en biparietale omtrek. Een verminderde groei snelheid van de buik omtrek is geassocieerd met een ongunstige neonatale uitkomst. Neonaten met een geboortegewicht lager dan verwacht op basis van de buik omtrek op 20 weken hadden meer afwijkende neonatale uitkomsten, ziekenhuis en NICU opnames. Dit hoofdstuk benadrukt het belang van een derde trimester scan om groei snelheden te berekenen.

Hoofdstuk 3 evalueert de waarde van het toevoegen van foetale groei snelheid en eerste trimester maternale biomarkers aan de baseline screening, voor de voorspelling van SGA neonaten en AGA neonaten met verminderde groei. Het baseline model bestaat uit maternale karakteristieken, obstetrische voorgeschiedenis, $\beta$-hCG, PAPP-A en een enkele foetale buik omtrek meting op 32 weken. SGA neonaten vergeleken met AGA neonaten hadden hogere bloed concentraties sFlt-1 en een hogere sFLt-1/PIGF ratio in het eerste trimester. SGA neonaten met verminderde groei lieten lagere PAPP-A en $\beta$-hCG, hoger sFlt-1 en een hogere sFLt1/PlGF ratio zien vergeleken met AGA neonaten met een normale groei.

De toevoeging van groeisnelheden of biomarkers aan het baseline mode verbeterde de voorspelling van SGA neonaten. De combinatie van baseline karakte- 
ristieken, groei snelheid en placenta biomarkers gaf de beste voorspelling voor SGA. We concludeerden dat het toevoegen van foetale groei snelheid en placenta biomarkers aan de baseline karakteristieken de predictieve kracht ten aanzien van SGA neonaten verbeterd. Het combineren van deze voorspellers zou mogelijk AGA neonaten met verminderde groei snelheid herkennen en diegene met een verhoogd risico op een afwijkende neonatale uitkomst.

Hoofdstuk 4 laat de longitudinale verandering van biomarkers in vrouwen met vroeg en laat-ontstane placenta dysfunctie zien. De longitudinale veranderingen in biomarkers vergeleken met een een-punts meting zijn beschreven. Vroeg-ontstane placenta dysfunctie was gedefinieerd als placenta-gemedieerde complicaties (foetale groei restrictie, preeclampsie en abruptio placentae) gediagnosticeerd voor 34 weken zwangerschapsduur. Laat-ontstane placenta dysfunctie was gedefinieerd als preeclampsie of zwangerschaps geïnduceerde hypertensie, gediagnosticeerd na 34 weken zwangerschapsduur. Vrouwen met vroeg-ontstane placenta dysfunctie hadden een geringere toename in PlGF en een grotere stijging in sFlt- 1 tussen 12 en 30 weken zwangerschap vergeleken met de referentie groep zonder placenta dysfunctie. De laat-ontstane groep liet geen verschillen zien in longitudinale biomarker levels vergeleken met de referentie groep. Gedurende het tweede trimester had de sFlt-1/PIGF ratio een betere voorspelling voor de predictie van vroeg-ontstane placenta dysfunctie dan sFlt- 1 of PlGF alleen. Terwijl gedurende het derde trimester alle individuele biomarkers significant gerelateerd waren aan vroeg-ontstane placenta dysfunctie. Vanaf het eerste trimester, is de longitudinale verandering van sFlt-1/PlGF ratio al onderscheidend voor vroegontstane placenta dysfunctie. We concludeerden dat sFlt-1/PlGF ratio het beste voorspellend vermogen voor vroeg-ontstane placenta dysfunctie heeft. Longitudinale metingen zijn alleen bruikbaar voor vroeg-ontstane placenta dysfunctie in ons cohort. Laat-ontstane placenta dysfunctie is een heterogene ziekte en eerste trimester placenta biomarkers hebben een slecht voorspellend vermogen om patiënten met een hoog-risico op het ontwikkelen van een laat-ontstane placenta ziekte te identificeren.

In hoofdstuk 5 hebben we een systematisch review uitgevoerd bestaande uit 26 studies om longitudinale veranderingen in biomarker concentraties en optimale onderscheidende afkapwaarde voor PlGF en sFlt-1 in de screening op SGA neonaten te beschrijven. Er was een grote variëteit in studie design, uitkomst definitie, assay methoden en moment van screening. Dit maakt de studies erg heterogeen. Door het gebruik maken van specifieke intervallen van zwangerschapsduur, lieten we zien dat de grootste verschillen tussen deze biomarkers in de SGA en referentie groep werden gevonden in PlGF en na 26 weken zwangerschapsduur. Een gemiddelde predictieve voorspelling werd gevonden met een brede range; 
dit geeft de heterogeniteit in studies weer. Vanwege het kleine aantal studies met foetale groei restrictie als uitkomstmaat, was het niet mogelijk om optimale afkapwaarde van biomarker levels voor deze uitkomst te beschrijven. Meer onderzoek is nodig om te zien of deze biomarkers op zichzelf voldoende de mogelijkheid geven om te differentiëren in groei vertraging en hoe deze biomarkers in combinatie met andere relevante klinische en echografische parameters gebruikt kunnen worden in klinische routine diagnostiek.

Hoofdstuk 6 onderzoekt de aanwezigheid van maternale vasculair malperfusie (MVM) laesies in SGA en subgroepen van AGA neonaten. MVM laesies worden gezien als consequentie van suboptimale remodellering van maternale spiraal arteriën, leidend tot foetale groei vertraging. Er is weinig literatuur over de prevalentie en impact van MVM laesies in AGA zwangerschappen met een normale foetale groei. Onze studie heeft laten zien dat MVM laesies ook in placenta's van AGA neonaten voorkomen. De aanwezigheid van MVM laesies correleerde omgekeerd met geboortegewicht, zelfs in AGA neonaten.. De combinatie van maternale karakteristieken, foetale groei snelheid en placentaire biomarkers gaf de beste voorspelling voor de aanwezigheid van MVM laesies. Deze resultaten suggereren dat een subgroep van normaal wegende neonaten kenmerken van FGR kunnen hebben en daarmee mogelijk een verhoogd risico lopen op een afwijkende neonatale uitkomst. Foetale groei snelheid, placenta biomarkers en de aanwezigheid van MVM laesies zou deze hoog-risico foetussen kunnen identificeren.

Hoofdstuk 7 beschrijft de validatie van het fetal medicine foundation (FMF) eerste trimester risico predictie model voor SGA in onze cohort. We gebruikten het model om risico op à terme SGA en foetussen met verminderde groei snelheid te berekenen, gebruik makend van baseline screening en biomarkers. De performance van het FMF algoritme voor à terme SGA was niet anders tussen baseline screening ten opzichte van baseline screening gecombineerd met biomarkers en maternale gemiddelde bloeddruk. Modellen waren slecht gekalibreerd en onderschatten het risico op SGA en verminderde groei snelheid in onze studie populatie.

Hoofdstuk 8 laat de postnatale groei gedurende de eerste vijf levensjaren van SGA en AGA neonaten zien. De onderzochte kinderen waren geboren in het MUMC+ en hadden controles bij de Gemeentelijke Gezondheids Dienst (GGD). SGA neonaten hadden een kleinere lengte en een lager gewicht vergeleken met AGA neonaten tot 3-jarige leeftijd. Catch-up groei, gedefinieerd als het verschil in groei tussen 4 weken en 3 jaar, was niet verschillend tussen beide groepen. Echter, neonaten met verminderde foetale groei hebben een hoger risico op catch-up groei in lengte vergeleken met normaal groeiende foetussen. Dit suggereert een 
rol voor foetale groei snelheid in het voorspellen van foetale en daarop volgend postnatale groei en gezondheidsrisico's op volwassen leeftijd.

Hoofdstuk 9 zet algemene discussie punten uit een over de bevindingen in dit manuscript binnen de context van de recente literatuur. Daaropvolgend, is een reflectie van de definitie van foetale groei vertraging beschreven, vier conceptuele fenotypes van foetale groei patronen zijn bediscussieerd, gebaseerd op data gepresenteerd in deze thesis, gecombineerd met huidige kennis over biomarkers en placenta histologie. Praktische implicaties over predictie en monitoring van foetale groei snelheid en toekomst perspectieven zijn weergegeven. 

c) 


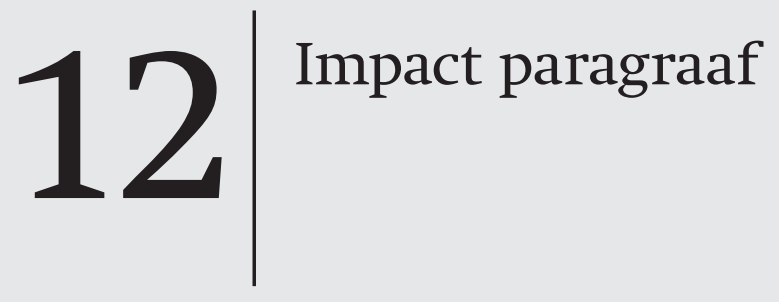





\section{Inleiding en relevantie}

Na een jarenlange afname van de perinatale sterfte in Nederland, lijkt deze sterftedaling de laatste tijd te stagneren en is er zelfs een stijging zichtbaar. Het recent verschenen Perined rapport, gebaseerd op de data van 2019 over perinatale sterfte in Nederland, wijst er op dat perinatale sterfte voor een groot deel samenhangt met de aanwezigheid van een of meer van de 'big 4'-risicofactoren: premature geboorte, congenitale afwijking, laag geboortegewicht en een lage Apgar-score. Daarnaast blijkt uit deze Nederlandse data van 2019 dat bij circa 28.000 van de in totaal 163.000 kinderen die geboren zijn na 24 weken zwangerschap één van deze 4 risicofactoren aanwezig is. Een premature geboorte en een laag geboortegewicht zijn daarbij de twee meest dominante risicofactoren. Bij circa 25.000 kinderen in Nederland (geboren na 24 weken zwangerschap) werd één van deze twee risicofactoren in 2019 vastgesteld. Dit geeft aan hoe belangrijk het is om onderzoek te doen naar de oorzaken van deze factoren tijdens de zwangerschap.

In dit onderzoek hebben we ons gericht op één van de oorzaken van een te laag geboortegewicht: foetale groei vertraging. Een te laag geboortegewicht kan het resultaat zijn van een verminderde groei tijdens de zwangerschap. Foetale groei vertraging wordt gebaseerd op het geschat gewicht van de foetus bij de groei echo's gedurende de zwangerschap. Op het moment dat de foetus of pasgeboren baby op één van deze momenten een laag geboortegewicht heeft, wordt deze als groei vertraagd beschouwd. Voor de beoordeling van het geboortegewicht wordt er gebruik gemaakt van landelijke (of internationale) groeicurven. Er is sprake van een laag geboortegewicht indien dit gewicht behoort tot de laagste 10 procent $(<\mathrm{p} 10)$ van de totale groep, rekening houdend met geslacht en zwangerschapsduur. Groei vertraging gaat gepaard met verhoogde risico's op een slechte uitkomst voor het kind. Wanneer groei vertraging wordt vastgesteld tijdens de zwangerschap, zal er een intensivering plaats vinden in de monitoring en controles van het kind tijdens de zwangerschap. Ook voor moeder kan een zwangerschap van een groei vertraagd kind tot problemen leiden, bijvoorbeeld door het ontstaan van een zwangerschapsvergiftiging.

Door de huidige manier van diagnostiek wordt er enkel naar grootte in plaats van foetale groei gekeken. Onze hypothese is dat met enkel de beoordeling van grootte, geen inschatting over de foetale groei kan worden gedaan. Hierbij wordt er namelijk mogelijk een groep gemist die wel een groei probleem heeft. Deze groep van foetussen heeft een normaal (geschat) geboortegewicht, gebaseerd op de grootte meting op één specifiek moment. Als naar deze groep echter gedurende de tijd (longitudinaal) wordt gekeken door middel van het maken van meerdere groei echo's en het vergelijken van de waardes van het geschatte geboortegewicht ziet men een afbuiging van de groei. Deze foetussen bereiken daardoor niet hun 
oorspronkelijke groei potentieel. Juist bij deze groep is het beter detecteren van groei vertraging van groot belang, zodat ze voldoende zorg krijgen en er zo nodig tijdig wordt ingegrepen.

Het hoofddoel van dit onderzoek is het beter kunnen opsporen van deze groep kinderen met 'gemiste' groei vertraging. De oorzaak voor foetale groei vertraging ligt vaak in het verminderd functioneren van de placenta. Er kan in de aanleg van de placenta iets mis zijn gegaan, waardoor de bloed- en zuurstofdoorstroming niet optimaal is, met het verminderd functioneren van de placenta tot gevolg. Als resultaat hiervan zal er een verminderde toevoer zijn van de navelstreng naar de foetus, wat kan leiden tot groei vertraging.

Voor het opsporen van de 'gemiste' groep groei vertraagde kinderen hebben we daarom zowel gekeken naar een andere wijze van het meten van groei vertraging als naar bloed onderzoek van de placenta. Voor het meten van groei vertraging hebben we de longitudinale groei in kaart gebracht middels het berekenen van groeisnelheden aan de hand van twee groei echo's. Er is voor die berekening gebruik gemaakt van zowel het geschatte gewicht als de buikomtrek van de foetus. Naast het geschatte gewicht heeft de buikomtrek van de foetus namelijk ook een grote voorspellende rol in de foetale groei. Op het moment dat de placenta minder goed gaat functioneren krijgt de groei van de hersenen prioriteit ten koste van andere organen, wat ook wel het "brainsparing-effect" wordt genoemd. Als gevolg hiervan blijft de foetale buikomtrek achter ten opzichte van de hoofdomtrek, wat de foetale buikomtrek een relevante en bruikbare indicator voor foetale groei vertraging maakt.

De werking van de placenta kan daarnaast ook opgemerkt worden door middel van bloedonderzoek doordat de placenta stofjes (biomarkers) afgeeft in het bloed van de moeder. Zowel groeisnelheden als placentaire biomarkers kunnen een rol spelen in het opsporen van een verminderde groei snelheid.

\section{Reflectie resultaten}

De belangrijkste resultaten in deze thesis bevestigen de hypothese dat er ook foetussen met groei vertraging zijn binnen een groep die nu als normaal groeiend wordt beschouwd op basis van het geboortegewicht (>p10). Deze foetussen met een verminderde groei snelheid hebben vaker een slechtere uitkomst bij de geboorte (12.4 vs. 3.9\%) en worden zelfs vaker opgenomen op de neonatale intensive care unit (6.2\% vs. 3.9\%) vergeleken met foetussen met een normale groei. Er is een significante associatie zichtbaar tussen groei snelheid in het tweede trimester en neonatale uitkomst.

Ondanks het normale geboortegewicht (>p10) worden ook in de placenta's van deze kinderen afwijkingen gezien die passen bij een verminderde werking van de 
placenta. Een model waarin groeisnelheden en biomarkers worden meegenomen voorspelt de aanwezigheid van deze afwijkingen in de placenta beter dan alleen aan de hand van maternale gegevens ( $82 \%$ vs. $25 \%$ ).

Het verminderen van de groeisnelheid zou mogelijk al eerder opgespoord kunnen worden door het meten van placentaire biomarkers in maternaal bloed. Bij foetussen met een verminderde groei worden al in het eerste trimester in het bloed van de moeder significant lagere waardes van PAPP-A gemeten, in vergelijking met foetussen met een normale groei snelheid. Dit verschil in biomarker waarde gerelateerd aan groei snelheid was zowel zichtbaar in foetussen met een normaal geschat geboorte gewicht (AGA) als foetussen met een laag geschat geboorte gewicht (SGA). Behalve PAPP-A, verschillen de waardes van PlGF en sFlt-1 gedurende de gehele zwangerschap tussen SGA en normaal groeiende zwangerschappen. Het is helaas nog niet mogelijk om met de huidige literatuur een afkapwaarde voor deze biomarkers op te stellen. Uit dit onderzoek blijkt ook dat het opsporen van groei vertraging of een laag geboorte gewicht enkel op basis van een predictie model in het eerste trimester echter nog onvoldoende is. We zien echter wel dat de voorspelling tijdens de zwangerschap op een laag gewicht bij de geboorte verbeterd kan worden (van $45 \%$ naar $74 \%$ ) door aan de basisscreening (gebaseerd op maternale karakteristieken en biometrie), zowel placentaire biomarkers als groeisnelheden in een later trimester toe te voegen. Deze foetussen met een verminderde groei snelheid behoeven net zoveel aandacht en controles als degene die nu gediagnosticeerd worden met een laag geboortegewicht. Het is hierbij enerzijds de uitdaging om de diagnose groei vertraging tijdig te stellen, anderzijds is het een uitdaging om het verdere beleid op te stellen ten aanzien van foetale bewaking tijdens de zwangerschap en het moment van bevallen. We hebben in deze studie gezien dat groei vertraging niet alleen een rol speelt bij de neonatale uitkomst, maar ook van invloed is op de groei van kinderen gedurende de eerste 3 levensjaren. Foetussen met een verminderde groeisnelheid hebben een hoger risico om in deze eerste jaren inhaal (catch-up) groei te laten zien. Deze inhaal groei is geassocieerd met een hoger risico op hart- en vaatziekten en het metabool syndroom. Dit betekent dat de gevolgen van een verminderde groei snelheid het gehele leven merkbaar kunnen zijn.

\section{Wetenschappelijke impact}

De resultaten in dit onderzoek laten zien dat foetale groei vertraging een dynamisch proces is wat op verschillende manieren in beeld kan worden gebracht. Dit onderzoek benadrukt ook dat een 'simpele' afkapwaarde van geboortegewicht $(<\mathrm{p} 10)$ een groep foetussen met groei vertraging mist. 
Juist het missen van deze groep leidt tot onderschatting van de aanwezigheid van groei vertraging, wat leidt tot toegenomen risico's van perinatale morbiditeit en mortaliteit. Groei vertraging blijft een heel heterogeen ziekte beeld, dit blijkt ook uit de review in deze thesis. De resultaten van deze studie zijn gepresenteerd op meerdere internationale congressen en gepubliceerd in internationaal peerreviewed journals. De publicaties die onderdeel zijn van deze thesis, geven meer inzicht in de oorzaken en gevolgen van groei vertraging. Daarnaast hebben ze een bijdrage geleverd aan de wetenschappelijke discussie over de definitie en detectie van groei vertraging. Recent is groei snelheid ook een onderdeel geworden in de consensus definitie van Gordijn et al. over foetale groei vertraging. Hierdoor vindt de transitie van wetenschappelijke resultaten naar klinische toepasbaarheid plaats.

\section{Doelgroep en maatschappelijke impact}

Door dit wetenschappelijk onderzoek hebben we een beter inzicht gekregen in het opsporen van groei vertraging. Deze resultaten kunnen de bewustwording en aandacht voor afbuigende groei vergroten met als gevolg intensivering van controles bij zwangerschappen met een afbuigende groei. Door hier meer bewustwording voor te creëren, kan worden bijgedragen aan het terugdringen van perinatale sterfte.

Deze onderzoeksresultaten zijn relevant voor zowel de zwangere vrouw als voor haar verloskundig zorgverlener. Voor gynaecologen, echoscopisten en verloskundigen is het belangrijk om alert te zijn op aanwijzingen voor groeivertraging gedurende de zwangerschap. Daarnaast is het belangrijk om op de hoogte te zijn van de gevolgen van (gemiste) groei vertraging. Deze zorgverleners kunnen tijdens richtlijn- en casuïstiekbesprekingen op de hoogte worden gebracht over nieuwe publicaties over het definiëren van groei vertraging. Ook binnen het verloskundig samenwerkingsverbanden (VSV's) kunnen deze bevindingen worden besproken. Daarnaast is het tijdens perinatale audits ook belangrijk om aandacht te besteden aan de groei van de foetus in relatie tot de neonatale uitkomst.

De laatste tijd is er meer aandacht voor predictie modellen om vroeg in de zwangerschap een inschatting te maken over de ontwikkeling van groei vertraging op basis van maternale karakteristieken en obstetrische voorgeschiedenis. De studie in deze thesis laat zien dat de toevoeging van biomarkers in predictiemodellen een waardevolle aanvulling zijn voor het nog beter definiëren en personaliseren van het risicoprofiel van de zwangere. Op basis van de resultaten uit een predictiemodel kunnen er preventie therapieën, zoals het gebruik van aspirine, met de zwangere vrouw worden afgestemd. 
Ook voor andere medisch specialisten heeft deze studie impact. Voor de kinderartsen is het rondom de bevalling goed om op de hoogte te zijn van een verwachte groei vertraging, gezien de vergrote kans op een slechte uitkomst voor de pasgeborene. Daarnaast spelen GGD-artsen en huisartsen een belangrijke rol bij de zorg tijdens de eerste levensjaren van het kind. Uit dit onderzoek blijkt dat groei vertraagde kinderen een inhaal groei tijdens de eerste jaren kunnen laten zien. Deze inhaal groei kan gepaard gaan met een verstoring in metabolisme. Daarnaast kan een groei vertraagd kind vroeg of laat problemen ontwikkelen op het gebied van mentaal, neuro-motorisch, psychosociaal en fysiek functioneren. Aandacht en alertheid hiervoor is belangrijk om vroegtijdige signalen te herkennen en waar mogelijk nadelige lange termijn gevolgen te voorkomen door tijdig ondersteuning te bieden.

Patiënten kunnen door middel van een folder en/of factsheet over het belang van groei van de baby geïnformeerd worden. Patiënt verenigingen zoals 'patiënt platform SGA' en 'Care4Neo' kunnen een rol spelen in de informatievoorziening naar ouders toe over de invloed van groei vertraging op het leven en het belang van follow-up. Tot slot gaan deze resultaten geïmplementeerd worden in een prospectieve studie, waarbij een register data zal linken van de zwangerschap aan de postnatale periode. 
c) 
List of publications

Dankwoord

Curriculum Vitae 



\section{CO-AUTHORS AND THEIR AFFILIATIONS}

Dr. N.O. Alers, Department of Obstetrics and Gynecology, Maastricht University Medical Centre, Maastricht, The Netherlands

Dr. S Al-Nasiry, Department of Obstetrics and Gynecology, Maastricht University Medical Centre, Maastricht, The Netherlands

S.E. El Bahaey, Department of Obstetrics and Gynecology, Maastricht University Medical Centre, Maastricht, The Netherlands

Prof. Dr. O Bekers, Department of Clinical Chemistry and Laboratory Medicine, Maastricht University Medical Centre, Maastricht, The Netherlands

Dr. J.A.P. Bons, Department of Clinical Chemistry and Laboratory Medicine, Maastricht University Medical Centre, Maastricht, The Netherlands

B.A.M. Conrads, Department of Obstetrics and Gynecology, Maastricht University Medical Centre, Maastricht, The Netherlands

W.P.T.M. van Doorn, Department of Clinical Chemistry and Laboratory Medicine, Maastricht University Medical Centre, Maastricht, The Netherlands

Prof. Dr. F.J.M. Feron, Department of Social Medicine, Maastricht University, Maastricht, The Netherlands

Dr. W.J.M. Gerver, Department of Pediatrics Endocrinology, Maastricht University Medical Centre Maastricht, The Netherlands

Dr. S.M.J. van Kuijk, Department of Epidemiology, Faculty of Health, Medicine and Life Sciences of Maastricht University, Maastricht, The Netherlands

Dr. A.W.D. Gavilanes, Department of Paediatrics, Maastricht University Medical Centre, Maastricht, The Netherlands. Department of Translational Neuroscience, School for Mental Health and Neuroscience (MHeNS), Maastricht University, Maastricht, The Netherlands.

Institute of Biomedicine, Facultad de Ciencias Médicas, Universidad Católica de Santiago de Guayaquil, Guayaquil, Ecuador. 
A. van Haren, Department of Obstetrics and Gynecology, Maastricht University Medical Centre, Maastricht, The Netherlands

Dr. D.M. Kimenai, Department of Clinical Chemistry and Laboratory Medicine, Maastricht University Medical Centre, Maastricht, The Netherlands

Prof. Dr. B.W.W. Kramer, Department of Pediatrics, Maastricht University Medical Centre, Maastricht, The Netherlands

D. Kramer, Department of Obstetrics and Gynecology, Maastricht University Medical Centre, Maastricht, The Netherlands

M.L. Kuin, Department of Obstetrics and Gynecology, Maastricht University Medical Centre, Maastricht, The Netherlands

K.C.M. Palm, Department of Obstetrics and Gynecology, Maastricht University Medical Centre, Maastricht, The Netherlands

Dr. H.C.J. Scheepers, Department of Obstetrics and Gynecology, Maastricht University Medical Centre, Maastricht, The Netherlands

Dr. C.A.H. Severens-Rijvers, Department of Pathology, Maastricht University Medical Centre, Maastricht, The Netherlands

R.R.G. Snellings, Department of Obstetrics and Gynecology, Maastricht University Medical Centre, Maastricht, The Netherlands

Prof. Dr. M.E.A. Spaanderman, Department of Obstetrics and Gynecology, Maastricht University Medical Centre, Maastricht, The Netherlands 


\section{LIST OF PUBLICATIONS}

M.L.E. Hendrix, J.H.C. Arits, R. Bannink, A.P.A. van Montfoort, C. Willekes, J.E. den Hartog, S. Al-Nasiry 'The choice for invasive prenatal tests after subfertility' Human Fertility (Camb). 2018 Sep 28:1-8.

M.L.E. Hendrix, S.M.J. van Kuijk, A.W.D. Gavilanes, D. Kramer, M.E.A. Spaanderman, S. Al-Nasiry 'Reduced fetal growth velocities and the association with neonatal outcomes in appropriate-for-gestational-age neonates'BMC Pregnancy Childbirth. 2019; 19(1):31.

M.L.E. Hendrix, J.A.P. Bons, R. Snellings, S.M.J. van Kuijk, O. Bekers, M.E.A. Spaanderman, S. Al-Nasiry 'Can Fetal Growth Velocity and First Trimester Maternal Biomarkers Improve the Prediction of Small-for-Gestational Age and Adverse Neonatal Outcome?' Fetal Diagnosis and Therapy. 2019;46(4):274-284.

M.L.E. Hendrix, K.C.M. Palm, J.A.P. Bons, S.M.J. van Kuijk, O. Bekers, M.E.A. Spaanderman, S. Al-Nasiry 'Prediction of placental syndrome in a high risk population using relative differences soluble fms-like tyrosine kinase-1 and placental growth factor measurements' Hypertension in Pregnancy. 2019;38(4):268-277.

M.L.E. Hendrix, J.A.P. Bons A. van Haaren, D. Kimenai, W.P.T.M. van Doorn, O. Bekers, M.E.A. Spaanderman, S. Al-Nasiry 'Systematic Review to Assess the Role of Soluble FMS-like Tyrosine Kinase-1 and Placenta Growth Factor Ratio in Prediction of SGA' Annals of Clinical Biochemistry. 2020;57(1):44-58.

M.L.E. Hendrix, J.A.P. Bons, N.O. Alers, C.A.H. Severens - Rijvers, M.E.A. Spaanderman, S. Al-Nasiry 'Maternal vascular malformation in the placenta is an indicator for fetal growth restriction irrespective of neonatal birthweight' Placenta. 2019;87:8-15.

M.L.E. Hendrix, S.M.J. van Kuijk, S.E. El Bahaey, W.J.M. Gerver, F.J.M. Feron, M.L. Kuin, M.E.A. Spaanderman, J.A.P. Bons, S. Al-Nasiry 'Postnatal growth during the first five years of life in SGA and AGA neonates with reduced fetal growth' Early Human Development. 2020 Dec;151:105199

M.L.E. Hendrix, W.M.J. van Doorn, B.A.M. Conrads, H.C.J. Scheepers, B.W. Kramer, M.E.A. Spaanderman, J.A.P. Bons, S. Al-Nasiry. 'External validation of a first trimester pregnancy algorithm predicting the risk for term SGA and reduced fetal growth velocity'. Submitted. 


\section{Oral presentations}

\section{ISUOG World Congress Berlin, October 2019}

- 'The association between fetal growth velocities and postnatal growth during the first five years after birth'

- 'Maternal vascular malformation in the placenta is an indicator for fetal growth restriction irrespective of neonatal birthweight'

\section{ISUOG World Congress Singapore, October 2018}

- 'Adding fetal growth velocity parameters to maternal biochemical biomarkers improves the detection of small-for-gestational-age neonates'

- 'Longitudinal sFlt-1, PlGF and sFlt-1/PlGF ratio measurements in predicting the development of placental syndrome in high-risk women'

\section{FNPS Meeting, Maastricht, June 2018}

- 'The additional value of fetal growth velocity parameters and maternal biochemical biomarkers for the detection of small-for-gestational-age neonates'

\section{Pélerin, Wetenschapssymposium MUMC+, October 2017}

- 'De associatie tussen suboptimale abdominale groei snelheid en een slechte neonatale uitkomst in appropriate-for-gestational age (AGA) neonaten'

\section{ISUOG World Congress Vienna, September 2017}

- 'The association between suboptimal fetal abdominal circumference growth velocity and adverse neonatal outcome in appropriate-for-gestational-age neonates'

\section{ISCOMS Congress Groningen, June 2017}

- 'The association between suboptimal fetal abdominal circumference growth velocity and adverse neonatal outcome in appropriate-for-gestational-age neonates'

\section{Pélerin, Wetenschapssymposium MUMC+, October 2016}

- 'Foetale abdominale groei snelheid als een voorspeller voor neonatale uitkomst en geboortegewicht in appropriate-for-gestational age (AGA) neonaten'

\section{ISUOG World Congress Rome, September 2016}

- 'Fetal abdominal circumference growth velocity as a predictor of neonatal outcome and birth weight in appropriate-for-gestational-age neonates' 


\section{Poster presentations}

\section{Zuyderland Wetenschapssymposium, June 2019}

- 'Foetale groeisnelheid in AGA neonaten'

\section{ISUOG World Congress Vienna, September 2017}

- 'The association between fetal abdominal circumference growth velocity and placental biomarkers in appropriate-for-gestational-age neonates'

\section{Mosa Conference, Maastricht University, June 2017}

- 'The association between suboptimal fetal abdominal circumference growth velocity and adverse neonatal outcome in appropriate-for-gestational-age neonates'

Wetenschapsdag MUMC+, Maastricht, May 2017

- 'The association between suboptimal fetal abdominal circumference growth velocity and adverse neonatal outcome in appropriate-for-gestational-age neonates' 



\section{DANKWOORD}

Wat ben ik blij en trots dat ik dit proefschrift heb mogen maken. Een mooie en uitdagende tijd, waarin ik de gelegenheid heb gekregen om vele enthousiaste mensen te leren kennen.

Dit proefschrift is tot stand gekomen dankzij de hulp, inzet en steun van veel collega's, vrienden en familie gedurende de afgelopen jaren. Zeker voor een promotie geldt 'alleen ben je sneller, maar samen kom je verder'. Dit dankwoord is dan ook gericht aan iedereen die mij gedurende mijn promotietraject heeft gesteund.

Allereerst gaat mijn dank uit naar alle zwangere vrouwen die vrijwillig hun zwangerschapsgegevens gedeeld hebben voor wetenschappelijk onderzoek.

Prof. Dr. M.E.A. Spaanderman, beste promotor, beste Marc, bedankt voor uw vertrouwen in mij, voor uw hulp en goede inhoudelijke feedback waarmee het onderzoek telkens weer naar een hoger niveau werd getild. Ik heb u ervaren als een zeer toegankelijke promotor met een heldere kijk. Ondanks alle drukte, nam $\mathrm{u}$ de tijd voor mij. Uw rustige en begripvolle houding, heeft een positieve invloed op mij en helpt me om het beste uit mezelf te halen.

Dr. S. Al-Nasiry, beste co-promotor, beste Salwan, bij jou is het allemaal begonnen tijdens mijn geneeskunde studie. Na een heel enthousiast gesprek waarin je over al jouw lopende onderzoeken vertelde, wist ik dat ik bij jou mijn onderzoekscarrière wilde starten. Vervolgens wees je mij de weg in de wondere wereld van wetenschappelijk onderzoek. Dat die eerste stappen uiteindelijk zouden leiden tot een presentatie op een internationaal congres, nadien een publicatie en uiteindelijk tot een echt proefschrift, had ik destijds nooit durven dromen. Je hebt me de afgelopen jaren zoveel geleerd, zowel op het gebied van onderzoek als in mijn persoonlijke ontwikkeling van geneeskunde student tot gynaecoloog in opleiding. Door jouw onuitputtelijke energie en enthousiasme ontstaan er steeds nieuwe uitdagingen, waar ik je ontzettend dankbaar voor ben. Ik kijk er naar uit om nog veel van je te mogen leren.

Dr. J.A.P. Bons, beste co-promotor, beste Judith. Bedankt dat je mij de kans bood om dit onderzoek samen met jou op te zetten en uit te voeren. Jij hebt altijd weer een verhelderende kijk en een luisterend oor. Jouw oprechte interesse in mij als persoon en je adviezen hebben me steeds geholpen om weer een stapje verder te 
komen met dit onderzoek. Ik hoop dat ik nog veel met je samen mag werken in de mooie projecten die we hebben opgezet.

Geachte leden van de beoordelingscommissie: Prof. Dr. J.G. Nijhuis, Prof. Dr. R.F.P.M. Kruitwagen, Prof. Dr. S.A. Scherjon, Prof. Dr. C.G.J. Sweep, Prof. Dr. L.J.I. Zimmermann. Ik waardeer het zeer dat $\mathrm{u}$ allen plaats wilt nemen in de beoordelingscommissie. Bedankt voor de beoordeling van het proefschrift.

Beste dr. Gavilanes, dank dat u vanaf het begin van de studie mee heeft gedacht over de neonatale uitkomsten en het verzamelen van deze data.

Beste Prof. Dr. Feron en dr. Gerver, bedankt voor jullie hulp met het verzamelen van de GGD data en de analyse van de groeicurves van de kinderen. Ik heb veel inspiratie opgedaan uit de brainstorm met jullie over de resultaten, daardoor hebben we er een mooi artikel van kunnen maken.

Beste Dr. van Kuijk, beste Sander, dankjewel voor al je hulp bij de statistische analyses. Keer op keer stond je klaar voor overleg momenten over de data analyse. Bedankt voor je geduld, fijne uitleg en mooie figuren.

Beste Prof. Dr. O. Bekers, bedankt voor uw waardevolle feedback op de manuscripten.

Beste Wim, Dorien en William. Wat ben ik blij dat Judith mij met jullie in contact heeft gebracht! Jullie hebben me enorm geholpen met de analyses van de biomarker data. William, ontzettend bedankt voor de ondersteuning en bemoedigende woorden bij het maken van de vele figuren en analyses, mede dankzij jou is het review-artikel geslaagd.

Beste Loe, bedankt voor je ondersteuning in het veilig verzenden van de placenta biopten voor mijn stage naar Cambridge. Wat was ik blij toen deze allemaal ijskoud arriveerden.

Beste Jos, dankzij jou hebben we heel veel biomarker gegevens kunnen verzamelen, dank voor het terugzoeken van de vele monsters en het uitvoeren van de analyses.

Beste Carmen, bedankt voor al jouw hulp en uitleg bij de beoordelingen van de placenta's. Leuk om ook nu nog steeds veel van je te mogen leren. 
Alle andere medeauteurs, ik wil jullie bedanken voor jullie kritische en waardevolle feedback op mijn manuscripten.

Beste echoscopisten, wat ben ik bij jullie enthousiast geworden over de echografie! Bij het verzamelen van de data heb ik bij meerdere echo's mee mogen doen op de PND. Ik kijk er naar uit om nog veel van jullie te mogen leren.

Onderzoekers, student-onderzoekers en oud-onderzoekers van de PERIM groep, bedankt voor de overleg momenten en de gezellige congressen samen.

Beste dames van het secretariaat, en in het bijzonder Monique en Manon, het was vaak een uitdaging om alle afspraken ingepland te krijgen. Toch kregen jullie het telkens weer voor elkaar. Hartstikke bedankt voor alle hulp en ondersteuning, het heeft me erg geholpen.

Miriam \& Lukasz, dankzij jullie heb ik naast wetenschappelijke ook klinische ervaring kunnen opdoen in Cambridge. Dank voor de ondersteuning en het mogelijk maken van deze fijne periode.

Beste Juliëtte, bedankt voor je ontwerpen voor dit proefschrift. Ontzettend fijn hoe jij alle ideeën in mijn hoofd kon weergeven in tekeningen op papier. Ik ben ontzettend blij met het resultaat.

Beste gynaecologen van het Zuyderland Medisch Centrum en Maastricht Universitair Medisch Centrum. Bedankt voor alles wat ik van jullie in de kliniek mag leren en voor de interesse in mijn promotietraject. Beste opleiders, beste dr. Wassen, dr. Smeets, dr. Willekes en dr. Slangen. Bedankt voor jullie interesse en ondersteuning in zowel mijn medische als persoonlijke ontwikkeling.

Beste collega arts-assistenten, dank voor de fijne opleiding samen en de gezelligheid met elkaar.

Lieve vrienden en vriendinnen, wat maakt het me gelukkig om omringd te zijn door zoveel lieve mensen. Dankjewel voor de momenten samen, die het leven zoveel leuker maken, even afschakelen van alles. Ik ben dankbaar voor de fijne vriendschappen en mooie herinneringen die we samen keer op keer maken. Daarbij wil ik Cécile en Stephanie speciaal bedanken. Bedankt dat jullie mijn paranimfen willen zijn en bedankt dat jullie altijd voor mij klaar staan. Jullie vriendschap is mij zeer dierbaar. 
Lieve familie, dank dat we er altijd voor elkaar zijn.

Lieve mama en papa, wat heb ik een geluk met jullie als ouders. Jullie hebben me altijd de ruimte gegeven om mijn eigen weg te gaan en op alle mogelijke manieren ervoor gezorgd dat ik hier nu sta. Ik ben ontzettend dankbaar voor het leven dat jullie mij geven. Bedankt voor de onvoorwaardelijke liefde, steun en het feit dat ik altijd op jullie kan rekenen.

Lieve Robbert, wat maak jij mij gelukkig. Ik geniet van onze tijd samen. Bedankt voor je liefde, support en vertrouwen om mijn dromen waar te maken. Ik kijk enorm uit naar onze avonturen die gaan komen. 


\section{CURRICULUM VITAE - OVER DE AUTEUR}

Manouk Hendrix werd op 27 januari 1994 geboren te Maastricht. Zij behaalde haar gymnasium diploma aan het Graaf Huyn College te Geleen. In 2012 startte zij met de opleiding geneeskunde aan de Universiteit van Maastricht. Al vroeg tijdens haar studie raakte ze betrokken bij het uitvoeren van wetenschappelijk onderzoek binnen de obstetrie. Ze bracht een periode in Cambridge door, waar zij placenta onderzoek verrichtte in het laboratorium van Prof. G.J. Burton. Daarnaast heeft ze tijdens een keuzecoschap ervaring opgedaan in het Addenbrooke's Hospital in Cambridge. Het wetenschappelijk onderzoek, uitgevoerd tijdens haar geneeskunde studie onder begeleiding van Dr. S. Al-Nasiry, Prof. Dr. M.E.A. Spaanderman en Dr. J.A.P. Bons, heeft de basis gevormd voor dit proefschrift. Ze presenteerde haar resultaten op meerdere internationale congressen.

Gedurende haar studie volgde ze een keuzecoschap obstetrie en gynaecologie in het Maastricht Universitair Medisch Centrum en was zij in het laatste jaar van haar studie werkzaam als semiarts binnen de obstetrie en gynaecologie in het Máxima Medisch Centrum te Veldhoven. Na haar afstuderen is zij aan de slag gegaan als arts niet in opleiding tot specialist gynaecologie in het Zuyderland Medisch Centrum te Heerlen. In januari 2020 is zij gestart met de opleiding tot gynaecoloog binnen het cluster Maastricht in het Zuyderland Medisch Centrum, opleider Dr. M.M.L.H. Wassen, plaatsvervangend opleider Dr. N.A.C. Smeets. Vanaf 2021 volgt zij het academisch deel van haar opleiding in het Maastricht Universitair Medisch Centrum, onder begeleiding van opleider Dr. C. Willekes en plaatsvervangend opleider Dr. B.F.M. Slangen. 